\title{
Inhaled nanomaterials
}

Citation for published version (APA):

Braakhuis, H. M. (2016). Inhaled nanomaterials: dose metrics for risk assessment. [Doctoral Thesis, Maastricht University]. Maastricht University. https://doi.org/10.26481/dis.20160127hb

Document status and date:

Published: 01/01/2016

DOI:

10.26481/dis.20160127hb

Document Version:

Publisher's PDF, also known as Version of record

\section{Please check the document version of this publication:}

- A submitted manuscript is the version of the article upon submission and before peer-review. There can be important differences between the submitted version and the official published version of record.

People interested in the research are advised to contact the author for the final version of the publication, or visit the DOI to the publisher's website.

- The final author version and the galley proof are versions of the publication after peer review.

- The final published version features the final layout of the paper including the volume, issue and page numbers.

Link to publication

\footnotetext{
General rights rights.

- You may freely distribute the URL identifying the publication in the public portal. please follow below link for the End User Agreement:

www.umlib.nl/taverne-license

Take down policy

If you believe that this document breaches copyright please contact us at:

repository@maastrichtuniversity.nl

providing details and we will investigate your claim.
}

Copyright and moral rights for the publications made accessible in the public portal are retained by the authors and/or other copyright owners and it is a condition of accessing publications that users recognise and abide by the legal requirements associated with these

- Users may download and print one copy of any publication from the public portal for the purpose of private study or research.

- You may not further distribute the material or use it for any profit-making activity or commercial gain

If the publication is distributed under the terms of Article $25 \mathrm{fa}$ of the Dutch Copyright Act, indicated by the "Taverne" license above, 


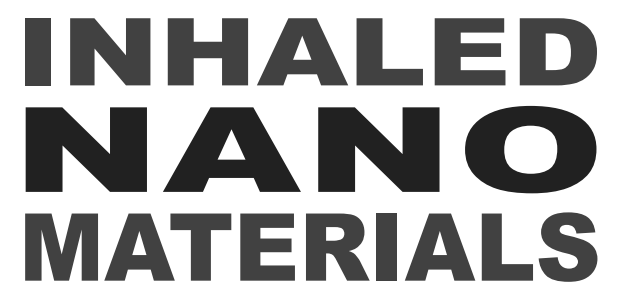

DOSE METRICS FOR RISK ASSESSMENT

Hedwig Magdalena Braakhuis 
(C) Hedwig Braakhuis, Utrecht, 2016 ISBN: 978-94-61 82-643-5

Layout: - Lein | redactie \& vormgeving, Utrecht Production: Off Page, $\mathrm{PhD}$ thesis production 


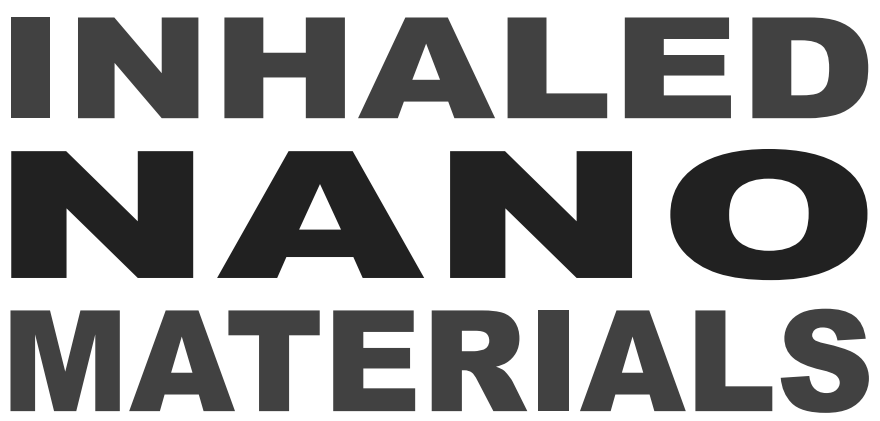

DOSE METRICS FOR RISK ASSESSMENT

Proefschrift ter verkrijging van de graad van doctor aan de Universiteit Maastricht, op gezag van de Rector Magnificus, prof. Dr. L.L.G. Soete volgens het besluit van het College van Decanen, in het openbaar te verdedigen op woensdag 27 januari om I 2:00 uur

door

Hedwig Magdalena Braakhuis

Geboren te Heerenveen

op 2 januari 1987 
Promotor:

Prof. Dr. H. van Loveren

Copromotor:

Dr. M. V. D. Z. Park, RIVM, Bilthoven

Beoordelingscommissie:

Prof. Dr. C. P. van Schayck (voorzitter)

Prof. Dr. Ir. B. Brunekreef (Institute for Risk Assessment Sciences, Utrecht)

Prof. Dr. P. Hoet (Katholieke Universiteit Leuven)

Prof. Dr. J. C. S. Kleinjans

Prof. Dr. P. J. Peters 


\section{CONTENTS}

$\begin{array}{ll}\text { Abbreviations } & 6\end{array}$

Chapter 1 General introduction Io

Chapter 2 Applications of inductively coupled plasma mass 44 spectrometry (ICP-MS) in biodistribution studies

Chapter $3 \quad$ Particle size dependent deposition and pulmonary 56 inflammation after short-term inhalation of silver nanoparticles

Chapter 4 Identification of the appropriate dose metric for pulmonary inflammation of silver nanoparticles in an inhalation toxicity study

Chapter 5 Progress and future of in vitro lung models to study IO4 translocation of nanoparticles

Chapter 6 Critical evaluation of different insert membranes to I 22 study translocation of nanoparticles

Chapter $7 \quad$ Use of in vitro models of increasing complexity to I 38 predict pulmonary toxicity of silver nanoparticles

Chapter 8 Effects of silver nanoparticles in an in vitro lung model I 58 exposed at the air-liquid interface

$\begin{array}{lll}\text { Chapter } 9 & \text { Summarizing discussion } & \text { I74 }\end{array}$

Valorisation paragraph $\quad$ I9I

Nederlandse samenvatting $\quad$ I95

$\begin{array}{ll}\text { References } & 205\end{array}$

Dankwoord 229

List of publications $\quad 233$

$\begin{array}{ll}\text { Curriculum vitae } & 235\end{array}$ 


\section{ABBREVIATIONS}

I6HBE

ADME

AF4

AFM

ALF

ALI

APS

BALF

BET

BSA

BSE

CC

CED

CLS

CMD

CPC

CPS

DCFH-DA

DLS

DMA

DMPO

DRC

EM

ESR

FCS

FFF

flow FFF

FRAS

GM-CSF

GSD

GSH

GSSG

HBSS

HDC

HF4

HR-ICP-MS

HUVEC

ICP-MS

ICP-OES

ICRP human bronchial epithelial cells

absorption, distribution, metabolism and excretion

asymmetric flow field flow fractionation

atomic force microscopy

artificial lysosomal fluid

air-liquid interface

aerodynamic particle sizer

bronchoalveolar lavage fluid

Brunauer, Emmett, and Teller

bovine serum albumine

back-scattered electrons

collision cell

critical effect dose

centrifugal liquid sedimentation

count median diameter

condensation particle counter

centrifugal particle sedimentation

2'-7'-dichlorodihydrofluorecein diacetate

dynamic light scattering

differential mobility analyser

5,5-dimethyl-I-pyrolline N-oxide

dynamic reaction cell

electron microscopy

Electron spin resonance

foetal calf serum

field flow fractionation

flow field flow fractionation

free radical analytical system

granulocyte macrophage colony stimulating factor

geometric standard deviation

reduced glutathione

oxidized glutathione

Hanks' balanced salt solution

hydrodynamic chromatography

hollow-fibre flow field flow fractionation

high resolution inductively coupled plasma mass spectrometry

human umbilical vein endothelial cells

inductively couple plasma mass spectrometry

inductively coupled plasma optical emission spectrometry

international commission on radiological protection 
IL

ISDD MODEL

KIT

LA

LAL

LDH

LM

LSGS

MALDI-TOF-MS

MALS

$\mathrm{MCH}$

$\mathrm{MCHC}$

MCP

$\mathrm{MCV}$

MIP

MMAD

MNS

MOI

MPPD model

MQ

MTS

MWCNT

NIST

NPS

NTA

OPS

PCLS

PIXE

PMA

PS

PVP

Q-ICP-MS

QSARS

RANTES

RIVM

RM

ROM

ROS interleukin

in vitro sedimentation diffusion dosimetry model

Karlsruhe Institute of Technology

laser ablation

limulus amebocyte lysate

lactate dehydrogenase

light microscopy

low serum growth supplement

matric-associated laser desorption/ionization time-of-flight mass spectrometry

multiple angle light scattering

mean corpuscular hemoglobin

mean corpuscular hemoglobin concentration

monocyte chemoattractant protein

mean corpuscular volume

macrophage inflammatory protein

mass median aerodynamic diameter

manufactured nanoparticles

micro-orifice impactor

multiple path particle dosimetry model

Milli-Q

3-(4,5-dimethylthiazol-2-yl)-5-(3-carboxymethoxyphenyl)-

2-(4-sulfophenyl)-2H-tetrazolium

multi-walled carbon nanotubes

National Institute of Standards

nanoparticles

nanoparticle tracking analysis

optical particle sizer

precision cut lung slices

particle-induced $\mathrm{X}$-ray emission

phorbol I2-myristate I3-acetate

polystyrene

polyvinylpyrrolidone

quadrupol inductively coupled plasma mass spectrometry

quantitative structure-activity relationships

regulated on activation, normal $\mathrm{T}$ cell expressed and secreted

Rijksinstituut voor Volksgezondheid en Milieu

reference material

reactive oxygen metabolites

reactive oxygen species 
SD

SDS

SE

SEM

SMPS

$\mathrm{sp}$

SPF

TEER

TEM

TEOM

THP-I

TLC

TLD

TNF standard deviation

sodiun dodecylsulfate

secondary electrons

scanning electron microscopy

scanning mobility particle sizer

single particles

specific pathogen free

transepithelial electrical resistance

transmission electron microscopy

tapered element oscillating microbalance

human acute monocytic leukemia cells

total lung capacity

total lung deposit

tumour necrosis factor 

In part published as: Physicochemical characteristics of nanomaterials that affect pulmonary inflammation

Hedwig M. Braakhuis ${ }^{\mathrm{I}, 2^{*}}$, Margriet V.D.Z. Park ${ }^{\mathrm{I}}$, Ilse Gosens ${ }^{\mathrm{I}}$, Wim H. de Jong ${ }^{\mathrm{I}}$, Flemming R. Cassee $^{\mathrm{I}, 3}$

Particle and Fibre Toxicology 20 I 4 April I I; I I: 8

${ }^{I}$ National Institute for Public Health and the Environment (RIVM), PO Box I, 3720 BA Bilthoven, the Netherlands

${ }^{2}$ Department of Toxicogenomics, Maastricht University, po Box 6I 6, 6200 MD Maastricht, the Netherlands

${ }^{3}$ Institute of Risk Assessment Sciences, Utrecht University, po Box 80.163, 3508 TD Utrecht, the Netherlands 
General introduction 


\section{DEFINITION AND USE OF NANOTECHNOLOGY}

Nanotechnology refers to the use of substances or structures on a very small scale to create novel products and applications. The term 'nano' is derived from the Greek vôvos, which means 'dwarf; and is used to refer to the size of very small structures. One nanometer is one billionth of a meter, which is in the size range of molecules. For example, I nm is 80.000 times smaller than the diameter of a human hair and $\mathrm{I} \mathrm{nm}$ is about the length that a fingernail grows in one second. Because of their small size, nanomaterials have a relatively large surface area, giving them unique properties that can be used to develop efficient solar cells, dirt-repellent textiles, anti-cancer drugs, and fast computers.

On I8 October 20 I I the European Commission adopted the 'Recommendation on the definition of a nanomaterial'. According to this recommendation, 'nanomaterial' means a natural, incidental or manufactured material containing particles, in an unbound state or as an aggregate or as an agglomerate and where, for $50 \%$ or more of the particles in the number size distribution, one or more external dimensions is in the size range $\mathrm{I} \mathrm{nm}$ - I00 nm (Commission, 20 I I).

Recently, many new applications of nanomaterials have been developed and the number of consumer products that claim to contain nanomaterials is increasing rapidly. In October 2013, the Nanotechnology Consumer Products Inventory contained 1628 products or product lines from over 30 countries, a growth of nearly $770 \%$ (from 2 I 2 to I628 products) since the Inventory was first released in March 2006 (Nanotechnologies, 20I4). There are 292 personal care products and I 54 cosmetic products on the market that claim to contain nanomaterials (Nanotechnologies, 20I4). The increase in the use of nanomaterials in products has raised concerns that such materials may introduce new hazards during occupational exposure, consumer exposure and/or environmental exposure.

\section{RISK ASSESSMENT OF NANOMATERIALS}

An adequate risk assessment of nanomaterials requires information on both the exposure and hazard of their component particles. This thesis focuses on the inhalation of nanomaterials as inhalation is considered to be an important route of exposure to nanoparticles (Maynard and Kuempel, 2005, Christensen et al., 2010), especially in occupational settings. Many products, such as sprays, may likewise lead to inhalation by consumers (Oomen et al., 20I I, Wijnhoven et al., 2010). In addition, the lungs are considered more vulnerable to chemicals and particles compared to the skin. With regard to hazard, numerous in vitro and in vivo studies have been conducted to determine whether inhalation of nanoparticles causes adverse effects. The most reported effect is pulmonary inflammation, largely indicated by an influx of neutrophils that can be 
observed in the bronchoalveolar lavage fluid in vivo and the induction of inflammatory cytokines in in vitro lung models (eg. Ji et al., 2007, Pauluhn, 20 I I, Sung et al., 2009, Yang et al., 2008, Oberdorster et al., 2000, Warheit et al., 2007c, Warheit et al., 2007b, Pauluhn, 2009a).

\section{DOSIMETRY OF NANOMATERIALS}

Dosimetry is one of the pillars in the risk assessment of chemicals, where doses and exposure limits are usually expressed in mass (g) per mass body weight or per volume of water or air $\left(\mathrm{L}_{\text {or }} \mathrm{m}^{3}\right)$. Several studies indicated that mass as dose metric is not suitable to express responses induced by manufactured nanoparticles (MNs) (Oberdorster et al., 2000, Stoeger et al., 2006, Horie et al., 20 I 2, Duffin et al., 2007, Kobayashi et al., 2009, Oberdorster et al., 2005a). The fact that mass is not a suitable dose metric also follows directly from the observation that at similar mass concentrations, nanomaterials behave different than their non-nano counterparts. This observation implies that the risk of the same mass of nano- and non-nanomaterial is different. For soluble substances a fixed number accounts for the number of dissolved molecules/atoms per unity of mass: Avogadro's constant $\left(6.02214179 \times \mathrm{IO}^{23} \mathrm{~mol}^{-1}\right)$. Therefore, the same mass of a soluble substance results in the same number of molecules/atoms in solution. For nanomaterials such a fixed number does not exist as particles may differ in size (distribution), shape etc. Therefore, the number of particles, surface area and volume of a nanomaterial may differ for the same unity of mass.

Following the above, a main problem for the risk assessment of nanomaterials is how to express and quantify the effective dose, for example when deriving an exposure limit. Nanomaterials are composed of primary and agglomerated particles that can vary in size, shape, charge, crystallinity, chemical composition and other characteristics, and this variety will increase even further in the future ((sCENinR) et al., 20Io). At present each nanomaterial with slightly different characteristics, e.g. $20 \mathrm{~nm}$ vs. $50 \mathrm{~nm}$, spherical vs. ellipses, coated vs. non-coated, should in principle be assessed separately for environmental and human health risks. All different physicochemical characteristics have been suggested to affect the toxicity of nanomaterials, but not all existing and emerging types of nanomaterials can be tested separately in studies to evaluate their safety. By analysing published data on inhalation of nanoparticles, we tried to identify trends regarding the characteristics of nanomaterials and pulmonary inflammation, as a key hazard indicator.

As the induction of pulmonary inflammation results from a combination of their deposition, clearance, and interactions in the lungs, the characteristics influencing one or more of these processes will be discussed in this chapter, using data of peer reviewed papers. Ultimately, these results can be used to design safer nanomaterials and to identify those that need to be investigated further in terms of their health risks. For the 
risk assessment of nanomaterials, knowledge on toxicity-determining characteristics will help to categorise nanomaterials into hazard groups according to these characteristics. Comparison across studies is often difficult due to the use of different experimental protocols and choice of endpoints, which largely influences the results. Therefore, our approach was to focus on investigations of multiple nanoparticles differing in one physicochemical characteristic within the same in vivo study. These studies are summarised in table $\mathrm{I}$.

Table 1. Inhalation studies investigating the effect of nanomaterial characteristics on lung deposition, clearance, and/or pulmonary inflammation. Studies are listed according to the nanoparticle characteristic studied, in alphabetical order. 


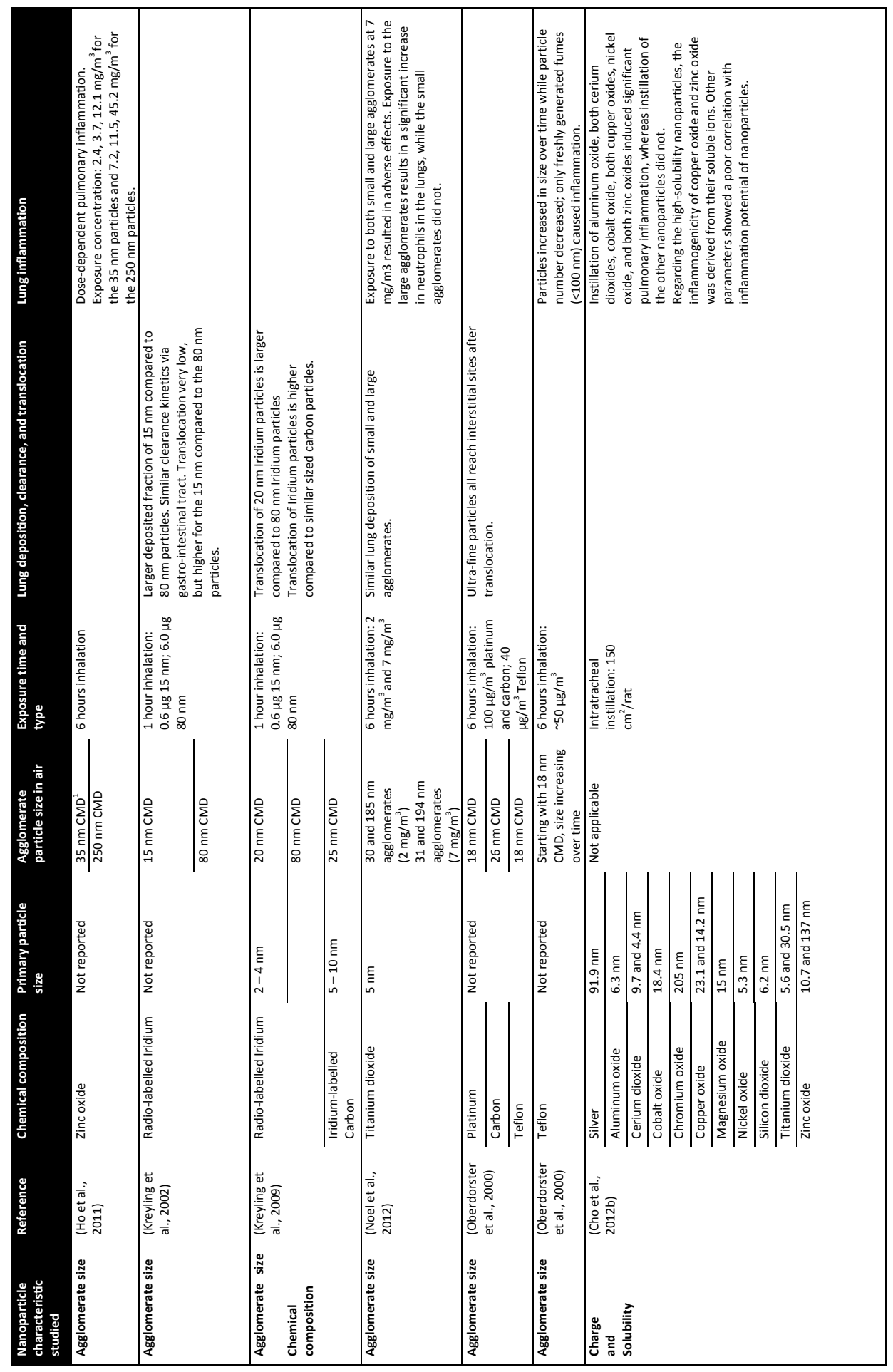




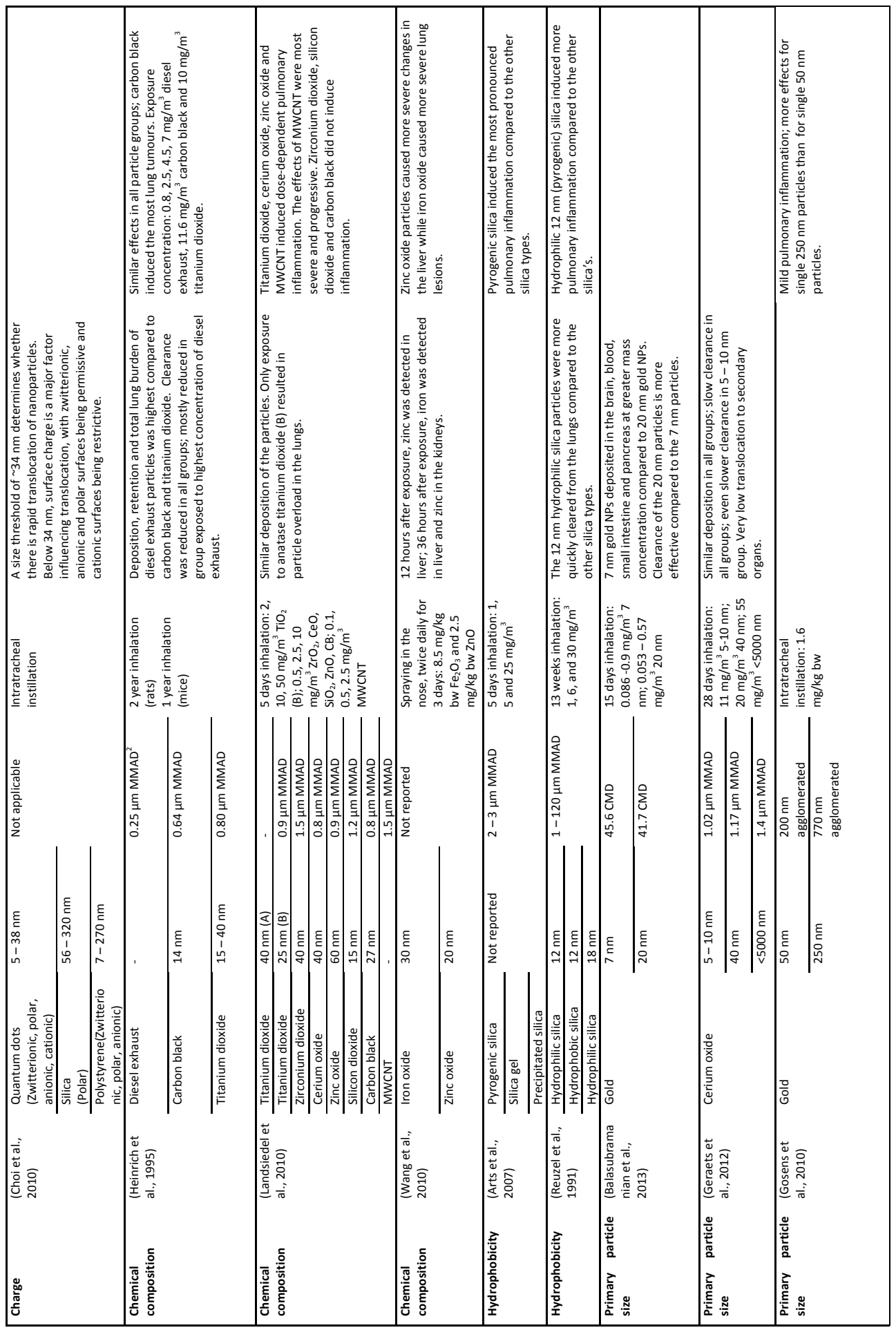




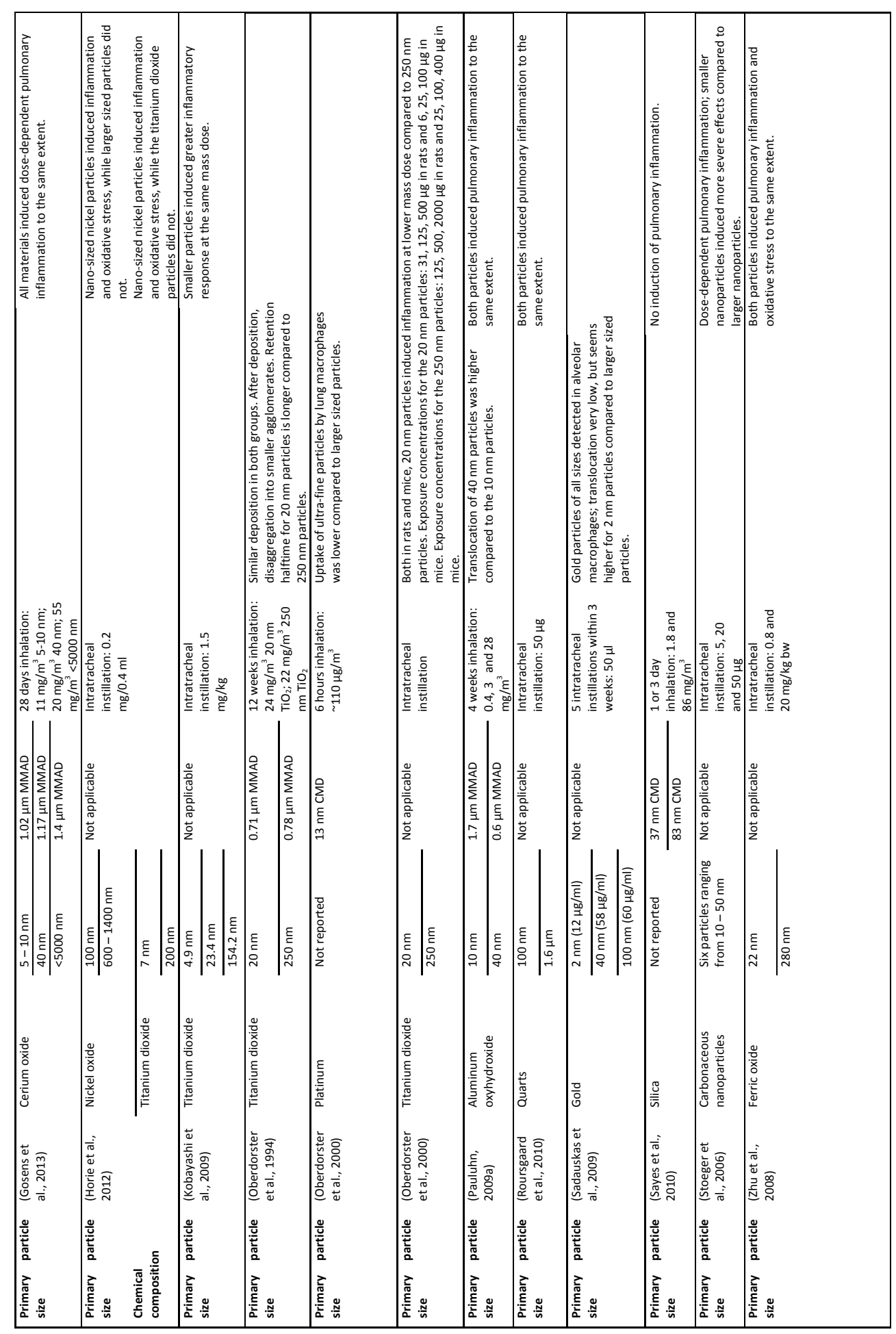




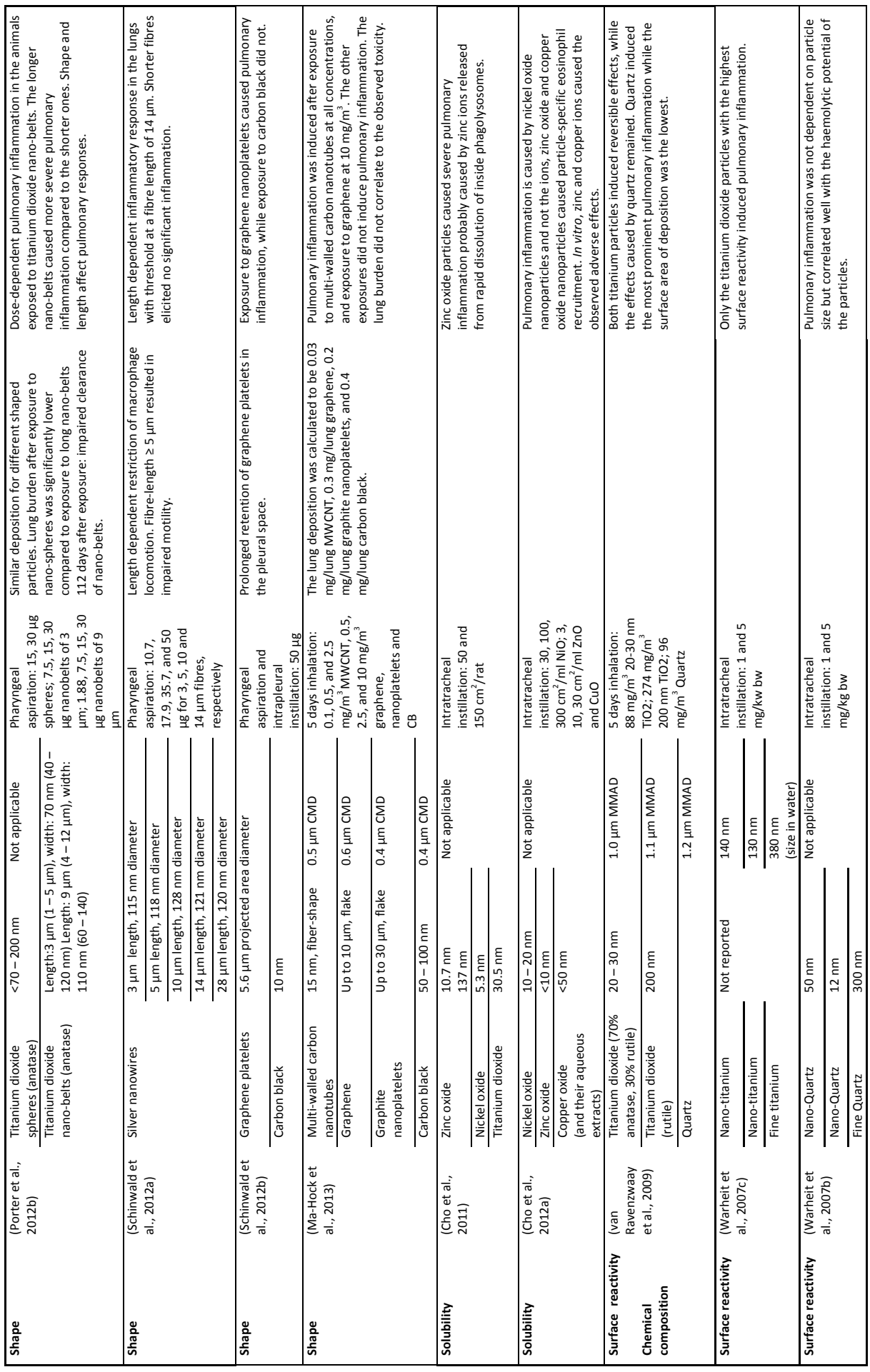

${ }^{\mathrm{I}} \mathrm{CMD}$ : count median diameter ${ }^{2}$ MMAD: mass median aerodynamic diameter 


\section{DEPOSITION OF NANOMATERIAL IN THE LUNGS}

A primary or individual nanoparticle (also called "ultrafine particle") has a mean primary diameter of $<\mathrm{IOO} \mathrm{nm}$, compared to $>\mathrm{I} \mu \mathrm{m}$ for a microparticle (also called "fine particle"). Primary particles tend to agglomerate, or aggregate, into larger particles. As they travel through the air from the point of generation to the point of exposure, the size of the primary and agglomerated particles determines their lung deposition pattern (figure I) (ICRP, I994, Asgharian et al., 2009, Carvalho et al., 20II). When nanoparticles are agglomerated in air, measurements of nanoparticle size will give the size of the agglomerates and not the primary particles (Veranth et al., 20 Io). The size of the agglomerates can be measured with different techniques, depending on the size of the agglomerates. Optical particle sizers (OPS) and aerodynamic particle sizers (APS) can measure the aerodynamic particle size ranging from $300 \mathrm{~nm}$ to $\mathrm{IO} \mu \mathrm{m}$ or $500 \mathrm{~nm}$ to $\mathrm{IO}$ $\mu \mathrm{m}$, respectively (Tritscher et al., Khlystov et al., 2004). The aerodynamic particle size is mostly given as a mass median aerodynamic diameter (MMAD). Differential mobility analysers (DMA) and scanning mobility particle sizers (sMPs) can measure the electrical mobility diameter of particles ranging from $2.5 \mathrm{~nm}$ to $\mathrm{I} \mu \mathrm{m}$, depending on the type of SMPS used. The electrical mobility diameter is mostly given as a count median diameter (CMD). The aerodynamic size depends not only on the physical size of the particles but also on the density of the particles, while the electrical mobility size depends on the physical size alone (Khlystov et al., 2004).

Nanoparticles with a primary or agglomerate particle size between Io and $\mathrm{I} 00 \mathrm{~nm}$ will deposit more efficiently in the alveolar region compared to particles with an agglomerate particle size between O.I and I $\mu \mathrm{m}$ (Asgharian et al., 2009, ICRP, I994, Carvalho et al., 20II, Cassee et al., 2002). In the alveoli the airflow is minimal, therefore, for nanoparticles between IO and $100 \mathrm{~nm}$, the mechanism of deposition in the lungs is diffusion (Oberdorster et al., 2005b). Several in vivo inhalation studies (Kreyling et al., 2002, Heinrich et al., 1995, Kreyling et al., 2009, Oberdorster et al., 2000) show that particles of smaller agglomerate size deposit more efficiently in the alveolar region than those of larger agglomerate size. Particles that differ in primary particle size but have the same agglomerate diameter show similar deposition fractions (Geraets et al., 20 I2, Landsiedel et al., 2010, Oberdorster et al., I994, Noel et al., 20I2). As stated above, for agglomerated nanoparticles with an aerodynamic size above $300 \mathrm{~nm}$, the density affects deposition. For these large agglomerates, increasing the density increases their deposition in the lungs, including in the alveolar region (figure I). It should be noted that the density of agglomerated particles is lower than the material density of the particles itself. Shape is also a factor. Primary and agglomerated nanoparticles occur in forms such as spheres, rods, fibres, wires, belts, triangles, and platelets. Shapes with a high aspect ratio, like fibres, have an aerodynamic size that is about three times their actual diameter; long fibres can deposit in the upper airways due to interception by touching the surface of the airways (Donaldson et al., 2010a, Sanchez et al., 20 I 2). 
For particles to induce pulmonary inflammation, they must deposit in the alveolar region. When the agglomerate size of nanoparticles is < IOO $\mathrm{nm}$ but above IO $\mathrm{nm}$, a considerable part of them will deposit in the alveolar region (about $30 \%$ of the particles) (Asgharian et al., 2009, ICRP, I994, Oberdorster, 1989). Although below $30 \mathrm{~nm}$, the deposition shifts from the alveoli more towards the tracheobronchial region.

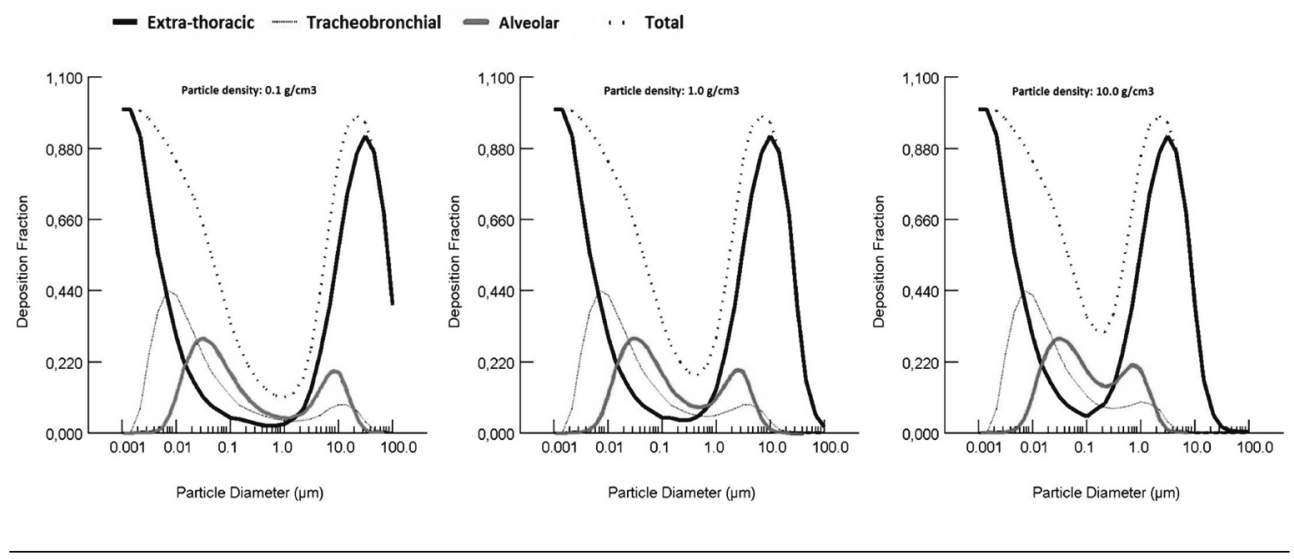

Figure 1. Deposition of particles in different regions of the lung depends on particle size and density. Particle size ranges from $1 \mathrm{~nm}$ to $100 \mu \mathrm{m}$, particle density tested: $0.1 \mathrm{~g} / \mathrm{cm}^{3}$ (left panel), $1.0 \mathrm{~g} / \mathrm{cm}^{3}$ (centre panel) and $10.0 \mathrm{~g} / \mathrm{cm}^{3}$ (right panel) (Simulation made in Multiple Pathway Particle Dosimetry Model V2.1 Copyright ARA 2009, based on human oronasalnormal augmenter breathing). The figure shows the deposition of inhaled particles in the extra-thoracic region (black line), the tracheobronchial region (thin grey line), and the alveolar region (thick grey line). In the alveolar region, the deposition is the highest for nanoparticles with a primary or agglomerate particle size between $10 \mathrm{~nm}$ and $100 \mathrm{~nm}$, regardless of the density. For particles with a primary or agglomerate size between 100 $\mathrm{nm}$ and $1 \mu \mathrm{m}$, the (agglomerate) density influences the deposition in the lungs: in this size range particles/agglomerates with a higher density will deposit more efficiently in the alveolar region compared to particles/agglomerates with a lower density.

Using the above information, one can predict the dose of nanomaterials in the lung using the Multiple Path Particle Dosimetry (MPPD) model. The model uses the morphology of the lung, respiratory conditions, and particle size (either CMD or MMAD), particle density, and exposure concentration to predict deposition in the various regions of the lung (Cassee et al., 2002). The exposure concentration determines the total amount of the nanoparticles that will deposit in the different regions of the lungs; it does not directly influence the deposition fraction in different regions of the lungs. It is important to note that the MPPD model gives an approximation of the deposition of particles in the lungs and results should be viewed with caution. For example, figure I shows extra-thoracic deposition of I $\mathrm{nm}$ particles and no tracheobronchial or alveolar deposition in humans during oronasal breathing, while there is substantial deposition of $\mathrm{I} \mathrm{nm}$ particles in the tracheobronchial region in humans during oral breathing (about 24\%) (Asgharian et al., 
2009). In addition, nanoparticles can be polydispersed in the air, resulting in a range of agglomerate sizes within a cloud of nanoparticles. When selecting CMD in the MPPD model, the polydispersity of the particles is not taken into account and the resulting lung deposition pattern should be interpreted as an estimation with uncertainties.

Another complicating factor is that nanoparticle agglomerate size changes over time by coagulation in air. The process is predominantly determined by Brownian motion and depends on the concentration of particles as they travel from site of generation to site of exposure; it also depends on the time required to reach the exposure site (Hinds, 1982). Nanomaterials are generated with a certain primary particle size but then tend to agglomerate, resulting in a lower number of particles with an increasing agglomerate size. The higher the particle number at generation, the faster these agglomerates are formed: nanoparticles of $30 \mathrm{~nm}$ primary size at a number concentration of $\mathrm{IO}^{7}$ particles/ $\mathrm{cm}^{3}$ are stable for a maximum of Io seconds, while the same nanoparticles at a number concentration of ${ }_{1} 0^{6}$ particles $/ \mathrm{cm}^{3}$ are stable for a maximum of $\mathrm{I}$ oo seconds (Kreyling, 2013). With longer travel times, the agglomerates increase in size. The speed and extent of agglomeration also depend on nanoparticle characteristics like surface charge, type of coating, and hygroscopicity. Particles with the same surface charge repel each other, whereas neutral particles more easily agglomerate. Similar, coatings can cause nanoparticles to repel or attract. Hygroscopicity describes particle response to water molecules in the environment, depending on the relative humidity. For example, they may attract molecules and grow many times their original size at increasing relative humidity (Varghese and Gangamma, 2009), or they may lose their own water content to evaporation, shrinking in size, at decreasing relative humidity.

When the influence of particle size is investigated, it should be measured as closely as possible to the site of exposure and not at the site of generation as a primary nanoparticle may constantly change its agglomerate size. After deposition of inhaled nanoparticles, agglomeration usually plays a minor role since the peripheral lung surface area is so large that the probability of two nanoparticles landing on each other is rather low for a diffusion-driven deposition. This is in contrast to deposition patterns of larger particles, which often congregate in 'hot-spots'. The binding kinetics of proteins as influenced by nanoparticle charge and other physicochemical properties of the surface is the more important mechanism after deposition. In animal studies the lung deposition can be measured, while in humans the deposition of particles in the different regions of the lungs can be modelled by the MPPD model. The results of the MPPD model should be interpreted with caution, as they are an estimation of what happens in reality.

\section{CLEARANCE OF NANOMATERIALS FROM THE LUNGS}

When nanoparticles are not exhaled, but deposited in the respiratory tract, there are several transport pathways to clear them. The sooner particles are cleared from the lungs, 
the smaller the likelihood that pulmonary inflammation will develop. The most prevalent mechanism for solid particle clearance in the alveolar region is mediated by alveolar macrophages, through phagocytosis (Oberdorster et al., 2005b). Once the macrophages have taken up particles, they move gradually toward the mucociliary escalator and are subsequently swallowed and cleared from the body through the gastrointestinal tract.

The retention time of particles in the lung depends on the deposition site and the interaction of particles with the inner lung surface. Particles that deposit in the conducting airways have a short retention time due to efficient mucociliary and cough clearance. The retention time increases when particles deposit deeper in the lungs, given the increased pathway length and decreased mucous velocity (Geiser and Kreyling, 20 I0). For microparticles, the retention half-time in the alveolar region is about 70 days in rats and up to 700 days in humans (Oberdorster et al., 2005b). For nanoparticles, the retention half-time tends to be longer because they can deposit in the alveolar region when their (agglomerate) size is between Io $\mathrm{nm}$ and $\mathrm{I}$ oo $\mathrm{nm}$. Even with a short retention time and complete phagocytosis by alveolar macrophages, pulmonary inflammation may still occur, as macrophages are well known for the release of pro-inflammatory mediators in response to the uptake of nanoparticles (Napierska et al., 20 I 2, Roberts et al., 20II).

If not cleared by phagocytosis, nanoparticles can reach pulmonary interstitial sites from which they are transported to the local lymph nodes. In addition, translocation of particles into the blood circulation can occur by crossing the lung barrier in the alveolar region (Borm et al., 2006). Subsequently, the particles are cleared from the body by the liver, gastrointestinal tract, or kidneys. However, translocated particles may be able to reach organs beyond the lung, where they can accumulate, and might cause damage. When the deposition of particles in the lung overwhelms the clearance mechanisms of the lung, this may result in a retained lung burden or accumulation of particles in the lung, which is greater than expected from linear kinetics. This situation is called lung particle overload (Morrow, I988, Morrow, I992, Pauluhn, 20 I I, Pauluhn, 20I0, Pauluhn, 2009b). In rats, particle overload may result in sustained inflammation, fibrosis and induction of lung tumours, but the evidence on whether this situation occurs in humans is inconclusive (ILSI, 2000).

For all these lung clearance and transport pathways, several nanomaterial characteristics are of influence.

\section{Clearance of nanomaterials: Particle size}

Size-dependent differences are important in the cascade of events leading to effective macrophage-mediated clearance. After deposition, agglomerates can disagglomerate into the primary particles (Oberdorster et al., 1994, Balasubramanian et al., 2013), or primary particles can agglomerate after contact with the lung lining fluid (Creutzenberg 
et al., 2012). At the site of deposition, the lung is thus exposed to large agglomerates, smaller agglomerates, or primary particles, as can be verified by transmission electron microscopy (TEM). Microparticles or large nanoparticle agglomerates, with a particle/ agglomerate size of $>\mathrm{I} \mu \mathrm{m}$, are easy for macrophages to phagocytize (Geiser and Kreyling, $2 \mathrm{O} \mathrm{IO}$ ), but single nanoparticles and small agglomerates are more difficult (Bakand et al., 20 I2, Muhlfeld et al., 2008a, Geiser, 2010), and the smaller the nanoparticles, the less efficient their clearance (Geiser et al., 2008). Within 24 hours after exposure, alveolar macrophages phagocytize only $20 \%$ of nanoparticles compared to $80 \%$ of microparticles (Oberdorster et al., 2005b). The nanoparticles that are not phagocytized are retained in the interstitium and in epithelial cells. Several in vivo inhalation studies report decreased clearance of nanoparticles from the lungs compared to larger-sized particles, resulting in increased retention time (Oberdorster et al., I994, Bakand et al., 20I 2, Muhlfeld et al., 2008a, Phalen et al., 2010). Increased retention gives nanoparticles the opportunity to translocate through the lung barrier. One study found low amounts $(<0.2 \%$ of the inhaled dose) of cerium oxide in secondary organs (Geraets et al., 20 I2). Another study reported no difference in clearance rate between $\mathrm{I} 5 \mathrm{~nm}$ and $80 \mathrm{~nm}$ radio-labelled iridium particles in rats. However, the translocation of the $\mathrm{I} 5 \mathrm{~nm}$ particles was higher compared to the $80 \mathrm{~nm}$ particles. Particles were found in secondary organs at fractions of $<0.002$ and $0.00 \mathrm{I}$ of deposited dose of the smaller and larger particles, respectively (Kreyling et al., 2002). This was confirmed in a follow-up study, in which translocation of $20 \mathrm{~nm}$ particles to secondary target organs was higher compared to $80 \mathrm{~nm}$ particles (Kreyling et al., 2009). Another group found that translocation of gold nanoparticles of $2 \mathrm{~nm}, 40$ $\mathrm{nm}$, and Ioo $\mathrm{nm}$ was very low, but greater for the $2 \mathrm{~nm}$ particles compared to the larger particles (Sadauskas et al., 2009). Similarly, after exposure to gold nanoparticles of $7 \mathrm{~nm}$ and $20 \mathrm{~nm}$ primary size (both having an agglomerate size of $45 \mathrm{~nm}$ ), the $7 \mathrm{~nm}$ particles were more subject to translocation and more heavily distributed in secondary organs than the larger particles. However, the $20 \mathrm{~nm}$ particles were detected at higher levels in the aorta and faeces (Balasubramanian et al., 20 I 3). In contrast to these findings, when rats were exposed via inhalation to aluminum oxyhydroxide nanoparticles of Io $\mathrm{nm}$ and $40 \mathrm{~nm}$ primary size ( $\mathrm{r} .7 \mu \mathrm{m}$ and $0.6 \mu \mathrm{m}$ aerodynamic size), the translocation of the larger particles was higher compared to the smaller particles. The particles translocated to the lung-associated lymph nodes but were not detected in any other secondary organ (Pauluhn, 2009a). The larger aerodynamic size of the Io nm particles probably resulted in a lower deposited fraction in the alveoli compared to the $40 \mathrm{~nm}$ particles, which might explain the higher translocation rate of the $40 \mathrm{~nm}$ particles.

Overall, single nanoparticles and agglomerates of $<100 \mathrm{~nm}$ are less efficiently phagocytized by alveolar macrophages compared to microparticles or large agglomerates of >I $\mu \mathrm{m}$ (Bakand et al., 20I2, Muhlfeld et al., 2008a, Phalen et al., 2010) and less efficiently cleared by mucociliary clearance (Geiser, 20 ro, Geiser et al., 2008). Increased retention of nanoparticles in the lung may damage the lungs or may result in the translocation of the nanoparticles to secondary organs. It is important to note that in general, clearance by translocation reported in the studies is very low, below $0.5 \%$ of the 
exposure concentration (Geraets et al., 20 I2, Kreyling et al., 2002, Kreyling et al., 2009, Sadauskas et al., 2009). Over time, this may still accumulate to significant amounts for persistent nanoparticles, but no studies are available to demonstrate this.

\section{Clearance of nanomaterials: Shape}

Clearance from the lungs is notably influenced by the shape of the particles (Yang et al., 2008). Rigid fibres may more readily be entrapped in the lungs compared to spherical particles (Vincent et al., 1985). In rat inhalation studies, longer fibres appeared to be more difficult to clear than shorter ones (Morgan et al., I 978). Fibres longer than about I 5-20 $\mu \mathrm{m}$ cannot be completely phagocytized by individual lung macrophages (McClellan and Henderson, 1995), resulting in frustrated phagocytosis in which adjacent cells attempt to phagocytize the same fibre (Donaldson et al., 20 I 2). In mice exposed to silver nanowires of different lengths via pharyngeal aspiration, shorter fibres with a length of 3,5 and Io $\mu \mathrm{m}$ could be completely phagocytized by alveolar macrophages whereas longer fibres with a length of I $4 \mu \mathrm{m}$ induced frustrated phagocytosis (Schinwald et al., 20 I 2a).

Exposure of mice to anatase titanium dioxide particles of various shapes (nanospheres, short nanobelts of $\mathrm{I}-5 \mu \mathrm{m}$, and long nanobelts of $4-\mathrm{I} 2 \mu \mathrm{m}$ ) resulted in a lung deposition of I $35 \mu \mathrm{g}$ both for the animals exposed to nanospheres and long nanobelts, but clearance was affected by particle shape. At I I 2 days after exposure, the lung burden was significantly lower in mice exposed to nanospheres $(45 \mu \mathrm{g}$ ) than in those exposed to long nanobelts (6o $\mu \mathrm{g}$ ) (Porter et al., 20 2 2b). Nanoplatelets can also impair clearance compared to spherical nanoparticles. After intrapleural instillation in mice, graphene nanoplatelets induced prolonged retention in the pleural space whereas carbon black did not (Schinwald et al., $20 \mathrm{I} 2 \mathrm{~b}$ ). The study shows that the aerodynamic diameter of graphene nanoplatelets is much smaller than the projected area diameter, giving the platelets the opportunity to deposit in the alveoli (Schinwald et al., 20I2b). In conclusion, long fibre-like or platelet nanoparticles are more readily entrapped in the lung and are more difficult to clear than shorter ones or spherical nanoparticles (Donaldson et al., 20 I 2, McClellan and Henderson, I995, Schinwald et al., 20 I2a, Porter et al., 20 I 2b, Schinwald et al., 20 I2b).

\section{Clearance of nanomaterials: Chemical composition}

Long-term exposure to diesel exhaust particles at concentrations of $0.8,2.5,4.5$ and 7 $\mathrm{mg} / \mathrm{m}^{3}$, carbon black at I I. $6 \mathrm{mg} / \mathrm{m}^{3}$, and titanium dioxide ( $80 \%$ anatase, $20 \%$ rutile) at $\mathrm{I} 0 \mathrm{mg} / \mathrm{m}^{3}$ resulted in impaired clearance from the lungs for all three types. After I 8 months of exposure and a recovery period of 3 months, lung clearance was still impaired and had not returned to normal levels. At that time point, the retained masses of the test materials were $47.7 \mathrm{mg} / \mathrm{lung}, 45.2 \mathrm{mg} / \mathrm{lung}$ and $37.8 \mathrm{mg} / \mathrm{lung}$ for diesel exhaust, carbon black and titanium dioxide, respectively. The strongest effect on lung clearance was caused by exposure to the highest concentration of diesel exhaust particles compared to similar concentrations of titanium dioxide and carbon black (Heinrich et 
al., 1995). The effects after short-term inhalation of 5 days were tested for two types of titanium dioxide (anatase or $80 \%$ anatase with $20 \%$ rutile), zirconium dioxide, cerium dioxide, zinc oxide, silicon dioxide, carbon black, and multi-walled carbon nanotubes (Landsiedel et al., 20 I0). All nanoparticles were tested at concentrations of 0.5, 2.5 and Io $\mathrm{mg} / \mathrm{m}^{3}$, except for titanium dioxide nanoparticles that were tested at 2 , IO and 50 $\mathrm{mg} / \mathrm{m}^{3}$ and multi-walled carbon nanotubes that were tested at $0.1,0.5$ and $2.5 \mathrm{mg} / \mathrm{m}^{3}$. Only exposure to anatase titanium dioxide, at the highest concentration tested, resulted in reduced lung clearance: the retained dose was $\mathrm{I} 635 \mu \mathrm{g}$ directly after 5 days inhalation and $\mathrm{I} 340 \mu \mathrm{g}$ at day $2 \mathrm{I}-29$ (Landsiedel et al., 2010). For the other nanoparticles tested, the retained doses in the lungs were at least a factor 4 lower, which might be explained by the lower exposure concentration.

Regarding translocation, the accumulation of carbon-iridium particles in secondary organs was lower compared to similar-sized iridium particles after inhalation (Kreyling et al., 2009). When rats were exposed to either $30 \mathrm{~nm}$ iron oxide particles $(8.5 \mathrm{mg} / \mathrm{kw} \mathrm{bw})$ or $20 \mathrm{~nm}$ zinc oxide particles $(2.5 \mathrm{mg} / \mathrm{kg} \mathrm{bw})$, there were differences in translocation rate and distribution in the body. After I 2 hours of exposure, zinc was detected in the liver, and after 36 hours, iron was detected in the liver and zinc was detected in the kidneys (Wang et al., 2010). These measurements made no distinction between particles and ions, but can likely be explained by differences in dissolution rates. Overall, clearance and translocation rates differ depending on the chemical composition of the nanoparticles.

\section{Clearance of nanomaterials: Surface charge}

Surface charge of nanoparticles may also influence their translocation rate. Endogenous proteins like albumin adsorb to the surface of charged nanoparticles, thereby increasing their hydrodynamic size, altering their surface charge (Lundqvist et al., 2008, Xia et al., 2006), and decreasing their surface reactivity and translocation rate (Choi et al., 20 Io). The higher the surface charge density, the more proteins are adsorbed (Gessner et al., 2002). On the other hand, zwitterionic or neutral organic coatings prevent adsorption of serum proteins (Choi et al., 2007). Examples are zwitterionic cysteine and polar PEG ligands that lead to rapid translocation of nanoparticles to the mediastinal lymph nodes (Choi et al., 20 Io). Overall, charged particles attract proteins and thereby reduce their translocation rate (Choi et al., 2010, Choi et al., 2007, Gessner et al., 2002). In addition, the attracted proteins can form a corona around the nanoparticles and alter their recognition and uptake by alveolar macrophages.

\section{Clearance of nanomaterials: Dissolution in physiological media}

Many nanoparticles are insoluble and retain their physical shape after deposition. Others, like zinc oxide, copper oxide, nickel oxide, iron oxide, silicon dioxide, and silver nanoparticles can dissolve at various rates. The epithelium of the respiratory tract is covered with a lining fluid, and materials that dissolve in this fluid are readily 
transferred to the blood (Kreyling, 1992). Only a few studies focused on the relation between particle dissolution and clearance from the lungs. One study suggests that after intratracheal instillation, the ability of metals to translocate from the lungs into the systemic circulation appears to be related to their solubility in water (Wallenborn et al., 2007). Another study showed that for cobalt oxide particles, the in vitro intracellular particle dissolution rate in alveolar macrophages was similar to the in vivo transfer to the blood (Kreyling, 1992). However, when the released ions precipitate and/or transform, they might not be readily transferred and remain in the lungs. Therefore, the correlation between intracellular particle dissolution and in vivo clearance by transfer to the blood is only valid for specific particles of which the intracellular dissolution rate is the limiting step in their clearance (Kreyling, I992). After deposition, some particles will be taken up by alveolar macrophages. Phagolysosomes inside the macrophages contain proteolytic enzymes, oxygen radicals, chelators, precipitators, and a low $\mathrm{pH}$ of about 5 , all of which may affect the engulfed particles. The low $\mathrm{pH}$ will increase particle dissolution. If particles release ions that destabilize the membrane of the lysosome, lysosomal content can leak and result in cell death, releasing the ions from the macrophages into the lungs. By affecting the barrier function of the lung epithelium, ions may enable intact nanoparticles to enter the bloodstream as well (Kreyling, I992, Koch and Stober, 200 I).

\section{PULMONARY INFLAMMATION INDUCED BY NANOMATERIALS}

After deposition in the alveoli, nanoparticles interact with the alveolar epithelium. Nanoparticles can escape clearance by alveolar macrophages resulting in prolonged interaction with the alveolar epithelium (Maynard et al., 2013). At a high deposited dose of nanoparticles, there is epithelial injury, the immune system will treat the presence of the particles as a threat and inflammation ensues. As a response to the epithelial injury, there is an influx of neutrophils into the alveolar region (Aarbiou et al., 2002, Zhou et al., 2012, Nicod, 1999). The epithelial cells generate chemotactic factors that stimulate the migration of macrophages (Barlow et al., 2005). A prolonged exposure of epithelial cells to nanoparticles may result in hyper-secretion of chemo-attractants into the alveolar space. It is possible that this may disrupt the normal chemotactic gradient within the lung and result in particle-laden macrophages remaining within the respiratory region instead of migrating to the mucociliary escalator for clearance (Donaldson and Borm, 2006).

The effect of nanomaterials after inhalation that is reported most often is that of pulmonary inflammation, characterized by an influx of polymorphonuclear neutrophils, which may be transient or persistent (Arts et al., 2007, Cho et al., 20I I, Cho et al., 20 I2a, Gosens et al., 2010, Heinrich et al., 1995, Ho et al., 20I I, Horie et al., 2012, Kobayashi et al., 2009, Landsiedel et al., 20 I0, Noel et al., 2012, Oberdorster et al., 2000, Pauluhn, 2009a, Porter et al., 20I2b, Reuzel et al., I99I, Roursgaard et al., 20 Io, Stoeger et al., 2006, van Ravenzwaay et al., 2009, Wang et al., 2010, Warheit et al., 2007b, Warheit et al., 2007c, Zhu et al., 2008, Gosens et al., 2013). At the 
cellular level, nanoparticle exposure can induce oxidative stress by the production of reactive oxygen species (ROS), which may be generated directly by particle structures in or near the cell or may arise more indirectly due to the effects of internalized particles on mitochondrial respiration (Xia et al., 2008) or the depletion of antioxidant species within the cell (Park et al., 2008). Oxidative stress can damage cells by peroxidising lipids, inducing inflammation, and altering proteins and DNA (Cheng et al., 2013). It can mediate a number of processes in the cells, such as apoptosis, DNA adduct formation, and pro-inflammatory gene expression (Donaldson and Poland, 20 I 2). All of these have been reported following exposure to some types and concentrations of nanoparticles (Donaldson et al., 20 iob, Moller et al., 20 io, Li et al., 2008, Landsiedel et al., 2009, Cho et al., 20 I 2a). Therefore, Ros production is considered the main underlying biochemical process in nanotoxicology, leading to inflammatory and other secondary processes that can ultimately cause cell damage and even cell death (Elsaesser and Howard, 20 I2, Nel et al., 2006, Cheng et al., 2013, Muhlfeld et al., 2008b). In the lung, persistent oxidative stress and inflammation after exposure to particulate matter are thought to cause fibrosis; in brain tissue, they are associated with neurodegenerative diseases (Brook et al., 20 Io, Simko and Mattsson, 2010).

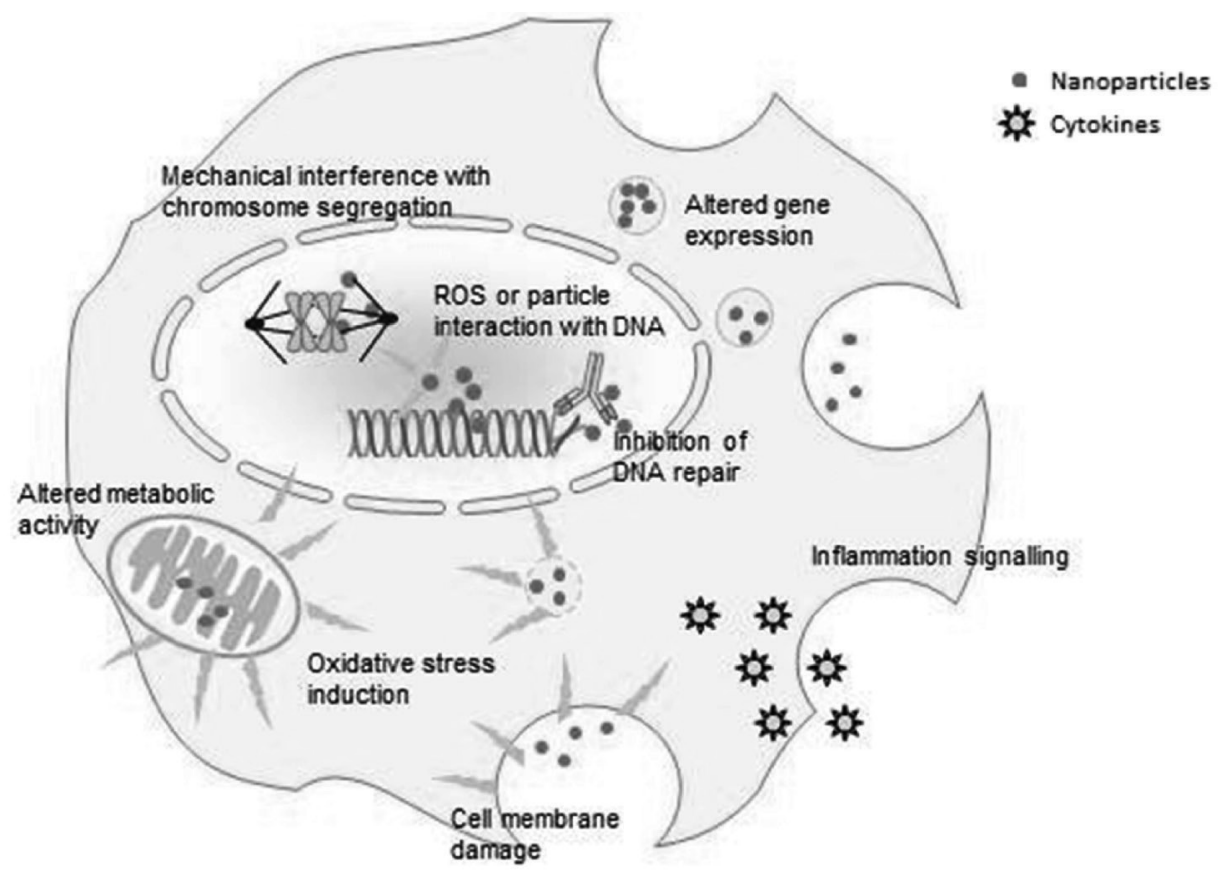

Figure 2. Suggested mechanisms underlying nanoparticle-induced responses at the cellular level. At sufficiently high or persistent levels nanoparticle-induced responses potentially lead to altered tissue function and damage. Uptake of nanoparticles by alveolar macrophages can result in the release of mediators and oxidative stress, which may lead to mitochondrial damage, damage to lipids and DNA, and inflammation (Colognato et al., 2012). 
The generation of ROs combined with proliferative signals at sites of persistent inflammation may also result in an accumulation of genetic defects. It is therefore important to determine which nanomaterial characteristics determine their persistence in the lung and their ability to cause inflammation. Figure 2 illustrates how nanoparticles can induce adverse effects at the cellular level. It should be noted that some of the mechanisms illustrated in figure 2 are based on in vitro and (in vivo) studies that use extremely high and therefore unrealistic concentrations. These results provide evidence for the mechanism behind the observed toxicity. However, they should be evaluated using lower concentrations that resemble realistic inhalation exposure conditions. The mechanistic pathways that operate at low realistic doses might be different from those operating at very high doses when the organism's defenses are overwhelmed (Oberdorster et al., 2005b).

\section{Pulmonary inflammation: Particle size and surface area}

Several in vivo studies compared the effect of particle size on pulmonary inflammation after inhalation or intratracheal instillation. Ultra-fine anatase titanium dioxide particles of $20 \mathrm{~nm}$ induced pulmonary inflammation after intratracheal instillation in rats and mice at lower mass concentrations compared to titanium dioxide particles of $250 \mathrm{~nm}$ (Oberdorster et al., 2000). Moreover, the onset of the inflammation was earlier in the $20 \mathrm{~nm}$ group. However, when the dose was expressed as surface area (measured by the method developed by Brunauer, Emmett, and Teller (BET) (Brunauer et al., I938)), the dose-response curves overlapped (Oberdorster et al., 2000), indicating that lung inflammation is determined by the total administered surface area. In another study, mice were intratracheally instilled with six types of carbon particles, with primary particle size ranging from to to $50 \mathrm{~nm}$ and specific surface area ranging from 30 to 800 $\mathrm{m}^{2} / \mathrm{g}$ (Stoeger et al., 2006). Results indicated that particle surface area, measured by BET method, is the best dose metric for responses induced by carbonaceous nanoparticles (Stoeger et al., 2006). In yet another study, however, the total surface area as measured by BET differed from the surface area calculated from particle size, resulting in different dose-response curves. The number of particles was thus considered a better dose metric to describe the effect of carbonaceous nanoparticles after inhalation, and not surface area (Wittmaack, 2007). These papers show that differences in measuring surface area of nanoparticles and differences in the analysis of experimental data can have a major influence on the results. Still, the studies show a clear effect of particle size in the induction of pulmonary inflammation, regardless if total surface area or particle number is the best dose metric to describe the dose-effect relationship.

Also in other studies, smaller particles caused more severe effects than larger particles when the same mass dose was administered. After intratracheal instillation at a concentration of $0.5 \mathrm{mg} / \mathrm{ml}$, nano-sized nickel oxide particles induced lung inflammation and oxidative stress, but micro-sized nickel oxide particles did not (Horie et al., 2012). In addition, several low-toxicity, low-solubility nanoparticles were tested in vivo by intratracheal 
instillation and in vitro on a human epithelial cell line. The data showed a clear relation between particle size and pulmonary inflammation as indicated by neutrophil influx and the induction of pro-inflammatory mediators; the smaller the particles, the greater the inflammatory response (Duffin et al., 2007).

To investigate the difference in effect between primary particle size and agglomerate particle size, anatase titanium dioxide particles of diverse primary and agglomerate sizes were tested. When primary particles of three sizes were intratracheally instilled in the lungs of rats at $1.5 \mathrm{mg} / \mathrm{kg} \mathrm{bw}$, smaller particles induced greater inflammation in the short-term, but for all groups the inflammation was resolved after one week, regardless of particle size. When anatase titanium dioxide particles with the same primary size but different agglomerate size were tested at $5 \mathrm{mg} / \mathrm{kg}$ bw, no clear relationship was observed (Kobayashi et al., 2009). When rats were intratracheally instilled with $50 \mathrm{~nm}$ primary gold particles, their agglomerates of $200 \mathrm{~nm}, 250 \mathrm{~nm}$ primary gold particles, or their agglomerates of $770 \mathrm{~nm}$, all four groups showed a mild inflammatory reaction at the tested concentration of $1.6 \mathrm{mg} / \mathrm{kg}$ bw. No differences were observed between single particles and their agglomerates (Gosens et al., 2010). However, when rats were exposed for 6 hours by inhalation to titanium dioxide particles with a primary size of $5 \mathrm{~nm}$ and an agglomerate size of $30 \mathrm{~nm}$ (small agglomerates) or $190 \mathrm{~nm}$ (large agglomerates), there was an effect of agglomerate size on pulmonary inflammation (Noel et al., 20 I 2). Exposures to both small and large agglomerates at $7 \mathrm{mg} / \mathrm{m}^{3}$ resulted in a lung burden of $5 \mathrm{I} .3$ and $5 \mathrm{I} .5 \mu \mathrm{g}$, respectively and induced increased lactate dehydrogenase (LDH) and oxidative stress markers. Exposure to the large agglomerates significantly increased the number of neutrophils in the lungs, while exposure to the small agglomerates did not (Noel et al., 2012).

As discussed earlier, the agglomerate size of nanoparticles can change over time. In one study, primary particles of ultra-fine Teflon fume increased in size over time while the particle number decreased, indicating agglomeration. The airborne time allowed 'aging,' and after 3.5 minutes of aging, agglomerated Teflon particles exceeded $100 \mathrm{~nm}$ and no longer caused toxicity; only freshly generated fumes caused pulmonary inflammation (Oberdorster et al., 2000).

Particles with a smaller primary size do not always induce more severe effects after inhalation than larger-sized particles in a similar dose. Ferric oxide particles of both 22 $\mathrm{nm}$ and $280 \mathrm{~nm}$ primary size induced dose-dependent pulmonary inflammation after intratracheal instillation of 0.8 and $20 \mathrm{mg} / \mathrm{kg} \mathrm{bw}$, and both sizes induced oxidative stress (Zhu et al., 2008). Aluminum oxyhydroxide particles of $\mathrm{I} 0 \mathrm{~nm}$ and $40 \mathrm{~nm}$ primary size and $\mathrm{I} .7 \mu \mathrm{m}$ and $0.6 \mu \mathrm{m}$ aerodynamic size both induced pulmonary inflammation after 4 weeks inhalation at the highest tested concentration $\left(28 \mathrm{mg} / \mathrm{m}^{3}\right.$ exposed concentration, I I $00 \mu \mathrm{g}$ of IO $\mathrm{nm}$ and $\mathrm{I} 800 \mu \mathrm{g}$ of $40 \mathrm{~nm}$ internal dose in the lungs), with no differences due to particle size (Pauluhn, 2009a). After exposure of mice to $50 \mu \mathrm{g}$ nano-sized and micro-sized quartz particles by intratracheal instillation, pulmonary inflammation was 
induced in both groups, without differences due to particle size (Roursgaard et al., 20 Io). Similarly, cerium oxide particles of 5 - $\mathrm{IO} \mathrm{nm}$ (I I mg/m $\left.\mathrm{m}^{3}\right), 40 \mathrm{~nm}\left(20 \mathrm{mg} / \mathrm{m}^{3}\right)$ and $<5000$ $\mathrm{nm}\left(55 \mathrm{mg} / \mathrm{m}^{3}\right)$ primary size induced dose-dependent pulmonary inflammation to the same extent after 28 days of inhalation, perhaps because their aerodynamic particle size was similar, at I.03 $\mu \mathrm{m} \mathrm{I.I7 \mu m} \mathrm{and} \mathrm{I.40} \mu \mathrm{m}$, respectively (Gosens et al., 20I3).

Overall, the relation between particle size and pulmonary inflammation is not straightforward, suggesting that other parameters also drive the response. With application of similar mass doses of microparticles and nanoparticles, the latter have a higher total surface area and total particle number, which may result in increased pulmonary inflammation. As discussed earlier particles of different sizes differ in patterns of lung deposition and clearance, which influence the actual internal dose that might have an adverse effect on the lungs. For some particles with the same chemical composition and probably within a limited size range, deposited particle surface area seems to be a better predictor for inflammation than exposure concentrations (Oberdorster et al., 2000, Stoeger et al., 2006, Horie et al., 20 I2, Duffin et al., 2007, Kobayashi et al., 2009).

\section{Pulmonary inflammation: Shape}

The high aspect ratio of long, thin and rigid carbon nanotubes has raised concern that these carbon nanotubes may induce pulmonary responses similar to asbestos (Castranova, 20 I I, Shvedova et al., 2005, Poland et al., 2008). Carbon nanotubes that are of a curly and tangled nature rather than being straight fibres will probably not induce these pulmonary responses. Several studies indicate that multi-walled carbon nanotubes (MWCNT) can induce severe pulmonary inflammation, possibly because of their fibre-shape (Porter et al., 20I 2a, Pauluhn, 2010, Ma-Hock et al., 2009, Mercer et al., 20I0, Porter et al., 2010, Ma-Hock et al., 2013). One group reported that highaspect-ratio single-walled carbon nanotubes were 23 -fold more inflammatory I day after aspiration in mice than an equal mass of spherical carbon black nanoparticles. As stated before, longer rigid fibres cannot be completely taken up by macrophages resulting in frustrated phagocytosis (Schinwald et al., 20 I2a, Donaldson et al., 20 I 2). This can lead to an inflammatory response by continuous release of pro-inflammatory mediators, recruitment of inflammatory cells, and generation of reactive oxygen species. In addition, it might disrupt the normal process of motility in the lungs, leading to accumulation of longer fibres in the lower respiratory tract (Donaldson et al., 20 Ioa, Coin et al., I994, Donaldson et al., 20 I2). In one study, only long nanofibres and long asbestos fibres elicited sustained inflammation in the pleural space, with extensive lesion formation and fibrosis along the parietal pleura (Murphy et al., 20 I I, Donaldson et al., 20I2). There is a cut-off value of $5 \mu \mathrm{m}$ for long fibres to induce effects in the pleural space (Schinwald et al., 20 I2c, Donaldson et al., 2012).

Besides fibres, other particle shapes can also influence the toxicity of nanoparticles. After pharyngeal aspiration in mice, at concentrations ranging from $\mathrm{I} .88$ to $30 \mu \mathrm{g}$, anatase 
titanium dioxide short nanobelts and long nanobelts induced dose- and time-dependent pulmonary inflammation while the nanospheres did not. In addition, there was some accumulation of long nanobelts in the interstitium suggesting increased interstitial access or impaired lymphatic clearance of particles with high aspect ratio (Porter et al., 20 I 2b). Wire-shaped silver particles induced a strong toxicity at similar particle mass, surface area and number compared to spherical particles on human epithelial cells in vitro. In contrast, the various lengths of wire did not affect the level of toxicity (Stoehr et al., 20II). These studies show that fibre-shaped, wire-shaped and nanobelt particles are more toxic to the lungs compared to spherical shaped nanoparticles (Shvedova et al., 2005, Porter et al., 2012b, Stoehr et al., 201 I).

\section{Pulmonary inflammation: Chemical composition}

It is plausible that, similar to conventional chemicals, the chemical composition of nanomaterials can influence their effect after inhalation exposure. Nanoparticles consisting of relatively toxic materials such as nickel and cobalt induce severe inflammation, as they have a high surface-specific activity and a large surface area per unit mass (Dick et al., 2003, Zhang et al., 1998). Ferric oxide and zinc oxide nanoparticles induced serious hepatic lesions in rats when sprayed directly into the nose twice daily over three days at 8.5 and $2.5 \mathrm{mg} / \mathrm{kg}$ bw, respectively. In general, the liver lesions were more severe in animals treated with zinc oxide than those treated with iron oxide. Pulmonary inflammation and lesions were likewise evident in both exposure groups and tended to be more severe in the group exposed to iron oxide (Wang et al., 2010). However, for both particle types, effects may have been caused at least in part by dissolved zinc or iron ions. In a long-term inhalation study, rats were exposed for 24 months and mice were exposed for I 2 months to similarsized aerodynamic particles of diesel exhaust at concentrations ranging from 0.8 to $7 \mathrm{mg} /$ $\mathrm{m}^{3}$, carbon black at I $1.6 \mathrm{mg} / \mathrm{m}^{3}$, or titanium dioxide at Io $\mathrm{mg} / \mathrm{m}^{3}$ (Heinrich et al., I995). After 24 months exposure, the retained doses in the lungs of rats were $63.9 \mathrm{mg} / \mathrm{lung}, 43.9$ $\mathrm{mg} /$ lung and $39.2 \mathrm{mg} / \mathrm{lung}$ for the highest exposed concentration of diesel exhaust, carbon black and titanium dioxide, respectively. Compared to controls, the mean lifetime of the rats was substantially shortened by exposure to carbon black and titanium dioxide, but not the diesel exhaust particles. Pulmonary inflammation and lesions were detected in all exposed animals. Particles of all three chemical compositions were detected in alveolar macrophages and in the alveolar region. After 6 months and 12 months of exposure, no lung tumours were found in the rats. After 24 months exposure and 6 months recovery, lung tumours were found in rats with all three exposures: $22 \%$ for diesel exhaust, $39 \%$ for carbon black, and $32 \%$ for titanium dioxide particles. It is remarkable that exposure to diesel exhaust resulted in the highest retained dose in the lungs but did not induce the highest tumour rate and did not shorten the lifetime of the rats. In mice, the tumour rate in the exposed groups did not differ from the controls (Heinrich et al., 1995).

After short-term inhalation of five days, differences were observed in the effects caused by nanoparticles of seven different chemical compositions (Landsiedel et al., 2010). 
All nanoparticles were tested at concentrations of $0.5,2.5$ and $\mathrm{IO} \mathrm{mg} / \mathrm{m}^{3}$, except for titanium dioxide nanoparticles that were tested at 2 , IO and $50 \mathrm{mg} / \mathrm{m}^{3}$ and multiwalled carbon nanotubes that were tested at $0.1,0.5$ and $2.5 \mathrm{mg} / \mathrm{m}^{3}$. Of these, titanium dioxide, cerium dioxide, zinc oxide, and multi-walled carbon nanotubes induced dosedependent pulmonary inflammation. For the first three, it was reversible at lower concentrations and partly reversible at the highest concentrations tested. The effects of multi-walled carbon nanotubes were irreversible and progressive. Exposure to zirconium dioxide, silicon dioxide, and carbon black induced no detectable inflammation at the tested concentrations. The nanoparticles that caused pulmonary inflammation were retained in the lungs at higher doses compared to the nanoparticles that did not induce pulmonary inflammation. After 5 days inhalation, the retained doses in the lungs were I $635 \mu \mathrm{g}$ titanium dioxide, $340 \mu \mathrm{g}$ cerium oxide, $428 \mu \mathrm{g}$ zinc oxide compared to 200 $\mu \mathrm{g}$ zirconium dioxide, and $93 \mu \mathrm{g}$ silicon dioxide, for the highest concentrations tested (Landsiedel et al., 2010). After intratracheal instillation at a concentration of $0.5 \mathrm{mg} / \mathrm{ml}$, nano-sized nickel oxide particles induced lung inflammation and oxidative stress while nano-sized titanium dioxide particles did not (Horie et al., 20I2). It must be noted that titanium dioxide nanoparticles occur in different crystal structures: anatase, rutile or a combination of both. Several in vivo and in vitro studies showed anatase titanium dioxide induce more adverse effects than rutile titanium dioxide (Hsiao and Huang, 20 I I, Warheit et al., 2007a, Sayes et al., 2006, Simon-Deckers et al., 2008, Grassian et al., 2007). According to these studies, chemical composition of the nanoparticles affects their potential to induce pulmonary inflammation, as would be expected from the different potency of conventional chemicals.

\section{Pulmonary inflammation: Surface charge}

Nanoparticles have different surface charges depending on the coatings, surfactants, and solvents used in production. In addition, they may acquire a corona of proteins after deposition in the lung. The surface charge can be measured by the zeta-potential, which is the electric potential created between the charged groups associated with the surface of a particle and the suspension medium. The zeta-potential reveals dynamic changes depending on the $\mathrm{pH}$ of the medium and the adsorption of proteins that form the corona. In most metal oxide nanoparticles, the zeta-potential is negative in a neutral $\mathrm{pH}$ of 7.4, predominantly positive in an acidic environment of $\mathrm{pH} 5.6$, and slightly negative when there is a corona of proteins or lung lining fluid (Donaldson et al., 20 I2). When nanoparticles encounter biological fluids containing macromolecules, they attract the oppositely charged ones to form the corona. The surface charge will change based on the adsorption of those molecules and proteins, thereby reducing the overall charge of the nanoparticles (Lundqvist et al., 2008, Xia et al., 2006). When they are phagocytized by alveolar macrophages, the proteolytic enzymes and acidic $\mathrm{pH}$ in the phagolysosomes may strip off all or part of the corona and reveal the naked surface of the particle, restoring its original zeta-potential (Wallace et al., I992). If the zeta-potential has a high positive value, the particle can bind to and damage membranes (Donaldson et al., 
20 I2). Positively charged nanoparticles are more easily taken up by lung cells, compared to neutral or negatively charged nanoparticles; they can thus remain in pulmonary cells for a long time, which may cause severe lung injury (Choi et al., 20ro, Zhang et al., 20I I). When nanoparticles with a high positive zeta-potential interact with the internal face of the lysosomal membrane, lysosomes can be destabilized, triggering cell death and inflammation (Hornung et al., 2008, Yazdi et al., 2010). Similarly, cationic nanoparticles are known to be more cytotoxic in vitro than neutral or anionic nanoparticles, causing lysosomal damage (Nel et al., 2009, Asati et al., 20ro, Cho et al., 20 I 2b, Nagy et al., 20I2). After intratracheal instillation of I 5 metal and metal oxide nanoparticles in mice at a concentration of $150 \mathrm{~cm}^{2} / \mathrm{rat}$, the ability of the particles to cause acute lung inflammation correlated linearly with their in vitro zeta-potential in an acidic environment (Cho et al., 20 I 2b). For the low-solubility particles, zeta-potential correlated best with the induced pulmonary inflammation (Cho et al., 20 I2b).

Overall, compared to neutral and negatively charged nanoparticles, positively charged nanoparticles can more easily be taken up by cells (Choi et al., 2010, Zhang et al., $20 \mathrm{II}$ ), leading ultimately to cell death and inflammation (Hornung et al., 2008, Yazdi et al., 2010).

\section{Pulmonary inflammation: Dissolution in physiological media}

Some nanoparticles can dissolve after deposition in the lungs, leading to formation of ions. When fast-dissolving nanoparticles are phagocytized by macrophages, the dissolution rate may be accelerated, leading to lysosomal destabilisation, cell death, and inflammation, dependent on the chemical identity of the ions that are released (Koch and Stober, 200I). For fast-dissolving nanoparticles, the effect is mainly driven by their chemical composition (Donaldson et al., 20 I2). Copper ions, zinc ions, and silver ions are known to have a toxic effect in vitro (Cho et al., 20 I I, Nel et al., 2009). Mice were intratracheally instilled with a panel of I 5 metal or metal oxide nanoparticles to relate their various physicochemical parameters to lung inflammation. Toxic ions, like copper and zinc caused destabilization of the lysosomal membrane (Cho et al., 20 I 2b). In the acidic conditions of phagolysosomes, nanoparticles of copper oxide, magnesium oxide, and zinc oxide showed rapid, complete dissolution, while nanoparticles like silver, cerium oxide, silica, and titanium dioxide showed minimal dissolution (Donaldson et al., 20I2). The pulmonary toxicity of nickel, zinc, and copper oxide nanoparticles and their aqueous extracts (containing only ions and no particles) were investigated both in vitro and in vivo (Cho et al., 20 I 2a). Results showed that the pulmonary inflammation induced by nickel oxide nanoparticles is caused by the particles and not by nickel ions in the aqueous extracts. For zinc oxide and copper oxide, the aqueous extracts induced effects similar to their corresponding nanoparticles in vitro. However, in vivo, zinc oxide and copper oxide nanoparticles caused particle-specific eosinophil recruitment that was not observed after administration of their aqueous extracts. In addition, exposure to the nickel and zinc oxide nanoparticles caused chronic effects that lasted up to four weeks. 
No aqueous extract caused such sustained inflammation, probably because soluble ions are rapidly cleared from the lungs.

It must be stressed that the dissolution rate of nanoparticles is not a constant factor but depends on particle size, coating, stability, manufacturing process, and biological environment. In vivo, released ions may be transported from the site of generation to other body parts, resulting in continued dissolution (and thus ion generation) of the residual nanoparticles. In vitro, dissolution may reach a maximum under static conditions. Especially for silver nanoparticles, the literature on their dissolution is contradictory. One study tested agglomeration, sedimentation, and dissolution of silver nanoparticles in biological media, finding that they did not dissolve in any of the tested fluids up to 96 hours incubation (Stebounova et al., 20I Ib). Other researchers report that silver nanoparticles indeed dissolve over time; the smaller the particles the faster they dissolve (Ma et al., 20I2). Whereas the effect of fast-dissolving nanoparticles probably depends on their chemical composition, the effects of slow- or partial-dissolving nanoparticles are more difficult to predict and will depend on the toxicity of the ions and of the particle that is retained (Donaldson et al., 2012).

\section{Pulmonary inflammation: Hydrophobicity}

Nanoparticles can be hydrophilic or hydrophobic, based mainly on their surface ligands, surfactants, or stabilizers (Nel et al., 2009). Hydrophobic nanoparticles are difficult to disperse in biological fluids and media, while hydrophilic particles easily disperse. However, hydrophobicity enhances the penetration ability of nanoparticles into cell membranes and nuclear pores through the hydrophobic effect (Naim et al., 2009, Cheng et al., 2013), which is the tendency of nonpolar substances to aggregate in aqueous solution and exclude water molecules (Chandler, 2005). To investigate the difference in effect between hydrophilic and hydrophobic nanoparticles, rats were exposed to three types of synthetic amorphous silica: $\mathrm{I} 2 \mathrm{~nm}$ particles of hydrophilic pyrogenic silica, I $2 \mathrm{~nm}$ particles of hydrophobic silica, and I $8 \mathrm{~nm}$ particles of hydrophilic precipitated silica. After inhalation exposure for I 3 weeks at I, 6 and $30 \mathrm{mg} / \mathrm{m}^{3}$, the most pulmonary inflammation was induced in the group exposed to $\mathrm{I} 2 \mathrm{~nm}$ hydrophilic silica, and the least inflammation in the group exposed to I $8 \mathrm{~nm}$ hydrophilic silica. This is interesting, as both particle types had similar specific surface areas. It must be noted that the $\mathrm{I} 2 \mathrm{~nm}$ hydrophilic silica dissolved quickly over time, which might have caused its inflammatory effects and subsequent fast clearance (Reuzel et al., I99I). In a 5-day inhalation study of three types of synthetic amorphous silica at I, 5 and $25 \mathrm{mg} / \mathrm{m}^{3}$, the pyrogenic silica (also known as fumed silica) induced the most pronounced pulmonary inflammation compared to silica gel and precipitated silica. The silica gel induced the least pulmonary inflammation. All three had a similar clearance rate (Arts et al., 2007). Like the I3week study, the 5 -day study showed that hydrophilic pyrogenic silica particles induced more severe pulmonary inflammation compared to other forms of silica, indicating that surface hydrophobicity/hydrophilicity can influence the effect of nanomaterials 
after inhalation. However, other particle characteristics, such as solubility, charge, and aggregation may also play a role.

\section{Pulmonary inflammation: Surface reactivity}

Chemical reactions and leakage of constituents occur at the surface of nanoparticles. The number of surface molecules increases exponentially when the particle decreases in diameter. Therefore, nanoparticles have a larger percentage of surface molecules compared to their 'bulk' counterparts (Oberdorster et al., 200 5b, Donaldson et al., 200 I). Surface reactivity is the potency of particles to react with the immediate environment by inducing reactive oxygen species (ROs), leakage of constituents, and other biochemical reactions. It depends on the chemical composition, shape, size, solubility, and surface area of particles (Knaapen et al., 2004, Moller et al., 2010), and is generally determined by measuring the induction of Ros, as pulmonary inflammation is thought to be caused by Ros generation at the nanoparticle surface (Elsaesser and Howard, 20 I 2, Nel et al., 2006, Cheng et al., 2013, Muhlfeld et al., 2008b).

In a 5-day inhalation study, rats were exposed to $20-30 \mathrm{~nm}$ titanium dioxide (mixture of $70 \%$ anatase and $30 \%$ rutile) at a concentration of $88 \mathrm{mg} / \mathrm{m}^{3}$, pigmentary $200 \mathrm{~nm}$ titanium dioxide (rutile) at a concentration of $274 \mathrm{mg} / \mathrm{m}^{3}$, or quartz particles at a concentration of $96 \mathrm{mg} / \mathrm{m}^{3}$. The exposure resulted in a retained dose in the lungs of 2025 $\mu \mathrm{g} 20-30 \mathrm{~nm}$ titanium dioxide, $9 \mathrm{I} 82 \mu \mathrm{g} 200 \mathrm{~nm}$ titanium dioxide, and $2190 \mu \mathrm{g}$ quartz at the end of exposure. The two titanium dioxide particles differ in their crystallinity and surface area; the pigmentary titanium dioxide and the quartz particles differ in chemical composition and surface reactivity. All three particle types induced pulmonary inflammation, but it was reversible after 14 days for both types of titanium dioxide while being not reversible for the quartz. The recovery from effects seemed faster for the smaller titanium dioxide nanoparticles compared to the larger ones, which could reflect the higher mass lung burden of the larger particles caused by their higher exposure mass concentration. Overall, the quartz particles induced the most pulmonary inflammation despite their deposited surface area being the smallest. Therefore, the authors conclude that surface reactivity is more important than surface area in nanoparticle toxicity (van Ravenzwaay et al., 2009). The same conclusion was reached when ultrafine titanium dioxide particles, differing in specific surface area and in surface reactivity, were tested in rats after intratracheal instillation at $\mathrm{I}$ and $5 \mathrm{mg} / \mathrm{kg}$ bw. Only the titanium dioxide particles with the highest surface reactivity induced pulmonary inflammation (Warheit et al., 2007c). However, those that induced no pulmonary inflammation were reduced in reactivity by a coating of silica or alumina. This finding implies that the rats were exposed to particles with different chemical composition and that toxicity is determined by the composition of the surface that comes in contact with a cell. Similar findings were reported in another study in which various types of quartz particles were intratracheally instilled in rats at $\mathrm{I}$ and $5 \mathrm{mg} / \mathrm{kg}$ bw. The intensity of the resulting pulmonary inflammation was wide-ranging and not dependent on particle size; surface 
reactivity determined the toxicity of nanoparticles rather than particle size (Warheit et al., 2007b). In vitro, quartz particles had a larger inflammatory potential compared to titanium dioxide and carbon black, although the quartz had a lower total surface area. The authors concluded that the greater ability of quartz to cause inflammation is related to its surface oxidative activity. For particles with a highly reactive surface like quartz, lower surface-area doses are required to induce pro-inflammatory responses (Monteiller et al., 2007).

Information on the surface reactivity of nanoparticles combines information on the effects of the chemical composition, shape, size, solubility, and surface area of the nanoparticles (Knaapen et al., 2004, Moller et al., 20 I0). In addition, several studies found a correlation between surface reactivity and pulmonary inflammation (van Ravenzwaay et al., 2009, Warheit et al., 2007b, Warheit et al., 2007c). Therefore, surface reactivity might be the most important nanoparticle characteristic determining their effect.

\section{Pulmonary inflammation: Methods to determine surface reactivity of nanomaterials}

Several methods are available to determine surface reactivity of nanomaterials. As pulmonary inflammation is thought to be caused by the generation of ROS at the nanoparticle surface (Elsaesser and Howard, 201 2, Nel et al., 2006, Cheng et al., 2013, Muhlfeld et al., 2008b), this process has been studied in both cell-free and cellular conditions. The oxidation potential of nanoparticles in cell-free conditions can be easily analysed by electron spin resonance (ESR) techniques. These techniques use a spintrapping agent to detect the nanoparticle-elicited generation of hydroxyl radicals in the presence of hydrogen peroxide. However, the process does not mimic the oxidation potential of the particles in the reducing environment of cells or extracellular fluid (Moller et al., 20I0). One study observed that the acellular potential of $20 \mathrm{~nm}$ silver nanoparticles to generate ROs was lower compared to larger silver nanoparticles, whereas its cellular potential was higher (Park et al., 20 I I). Another study observed that carbon black generated substantial amounts of Ros under cell-free conditions, but titanium dioxide nanoparticles did not. However, both showed a comparable dose-dependent capacity to produce intracellular ROs (Hussain et al., 2009). These results suggest that the generation of ROs might be an indirect effect of the interaction of the nanoparticles with cellular components. As it occurred in macrophages only at concentrations above those that reduce their metabolic activity, Ros generation may have been a secondary effect rather than causing the onset of cytotoxicity (Park et al., 20I I). Therefore, the inability of nanoparticles to produce Ros in cell-free systems does not rule out their potential to produce intracellular oxidative stress (Hussain et al., 2009). Measuring the intracellular induction of ROS after nanoparticle exposure in vitro might be a way to categorize nanoparticles into hazard groups. However, there is no validated in vitro assay available to test all types of nanomaterials. 
The intracellular induction of ROs can be measured using ESR techniques in combination with in vitro cellular exposure or using the 2'-7'-dichlorodihydrofluorecein diacetate (DCFH-DA) assay. The DCFH-DA assay uses a fluorescent probe to visualize the induction of ROs in cells after exposure to nanoparticles. Another method is the free radical analytical system (FRAS) assay that measures the formation of reactive oxygen metabolites (ROM) after exposure to nanoparticles. Besides measuring Ros generation, the surface reactivity of nanomaterials can be measured based on how well they serve the purpose for which they were designed. For example, some nanomaterials are added to a product for their catalytic potential, and others for their anti-bacterial activity or uv absorbance. For such nanomaterials, the surface reactivity required to catalyse reactions, kill bacteria, or absorb UV can be used to determine the actual surface reactivity of the materials. Other methods to measure surface reactivity are the erythrocyte haemolysis assay (Harington et al., I97I, Nolan et al., I98I) and the vitamin C yellowing assay (Warheit et al., 2007c, Rajh et al., 1999). The erythrocyte haemolysis assay measures the amount of haemoglobin released after exposure of red blood cells to nanoparticles, and the vitamin $\mathrm{C}$ assay measures the chemical reactivity of nanoparticles toward an anti-oxidant. Neither of the assays can measure the surface reactivity of all types of nanomaterials.

Box 1. Conclusions on the physicochemical characteristics of nanomaterials that affect pulmonary inflammation.

\section{Conclusions}

- The induction of pulmonary inflammation by nanomaterials depends largely on the extent of deposition in and clearance from the lungs.

- No single particle property can be identified as the most important in the induction of pulmonary inflammation by nanomaterials, as various properties affect different stages of the events leading to pulmonary inflammation.

- Surface reactivity might be the best predictor for a nanomaterial's potential to induce pulmonary inflammation.

- There is a lack of information on the potential effects of longterm inhalation exposure to persistent nanomaterials, in terms of a potential delayed onset of pulmonary inflammation and translocation to secondary organs. 


\section{SUMMARY AND CONCLUSIONS}

Although size has been put forward as an essential parameter to predict the pulmonary inflammation caused by nanomaterials, many other factors modulate the outcomes of toxicity studies. Our conclusions on the physicochemical characteristics of nanomaterials that affect pulmonary inflammation are shown in box I. One essential step in predicting the risk of adverse human health outcomes based on experimental studies is to elucidate the deposition and clearance of nanomaterials. These processes are largely driven by the physical characteristics of nanoparticles and need to be taken into account when investigating to what extent specific nanomaterial characteristics affect pulmonary inflammation. Agglomerate particle size and density are the most dominant of all the nanomaterial characteristics that affect lung deposition. Using these values as input, the dose of nanomaterials in the lung can quite accurately be predicted by the Multiple Path Particle Dosimetry (MPPD) model. However, it should be realised that other nanomaterial characteristics affecting lung deposition have not been investigated to the same extent, and results of the MPPD model should be interpreted with caution. Primary size, shape, chemical composition, charge, and dissolution rate can all affect clearance rate.

Many physicochemical characteristics of nanomaterials influence the severity of pulmonary inflammation, and no unifying metric can be identified based on the current available evidence. Some studies use rather high and unrealistic exposure concentrations, which might lead to lung particle overload conditions and severe adverse effects in laboratory animals, which will probably not occur under realistic in vivo exposure conditions in humans (ILSI, 2000). Results on the influence of primary particle size on pulmonary inflammation after inhalation are rather contradictory. Some studies report increased pulmonary inflammation after exposure to nanoparticles compared to larger micro-sized particles (Oberdorster et al., 2000, Stoeger et al., 2006, Horie et al., 20I 2, Kobayashi et al., 2009, Duffin et al., 2007), while others report no difference (Zhu et al., 2008, Pauluhn, 2009a). As discussed earlier, both the agglomerate size and the primary size of particles affect lung deposition, clearance, and translocation. Therefore, inhalation studies should measure the actual deposition and retention of particles in the lung, preferably as total mass, surface area, and number dose. By comparing the effects of these local doses for the different particle sizes, conclusions can be drawn about the role of size in pulmonary inflammation. For some particles of the same chemical composition (and probably within a limited size range), the deposited surface area seems to be a better predictor for inflammation than mass exposure concentrations (Oberdorster et al., 2000, Stoeger et al., 2006, Horie et al., 20 I 2, Duffin et al., 2007, Kobayashi et al., 2009). In addition, nanoparticles tend to agglomerate or even aggregate, changing particle size and available surface area. Information on the influence of particle agglomeration and aggregation on pulmonary inflammation is very limited. 


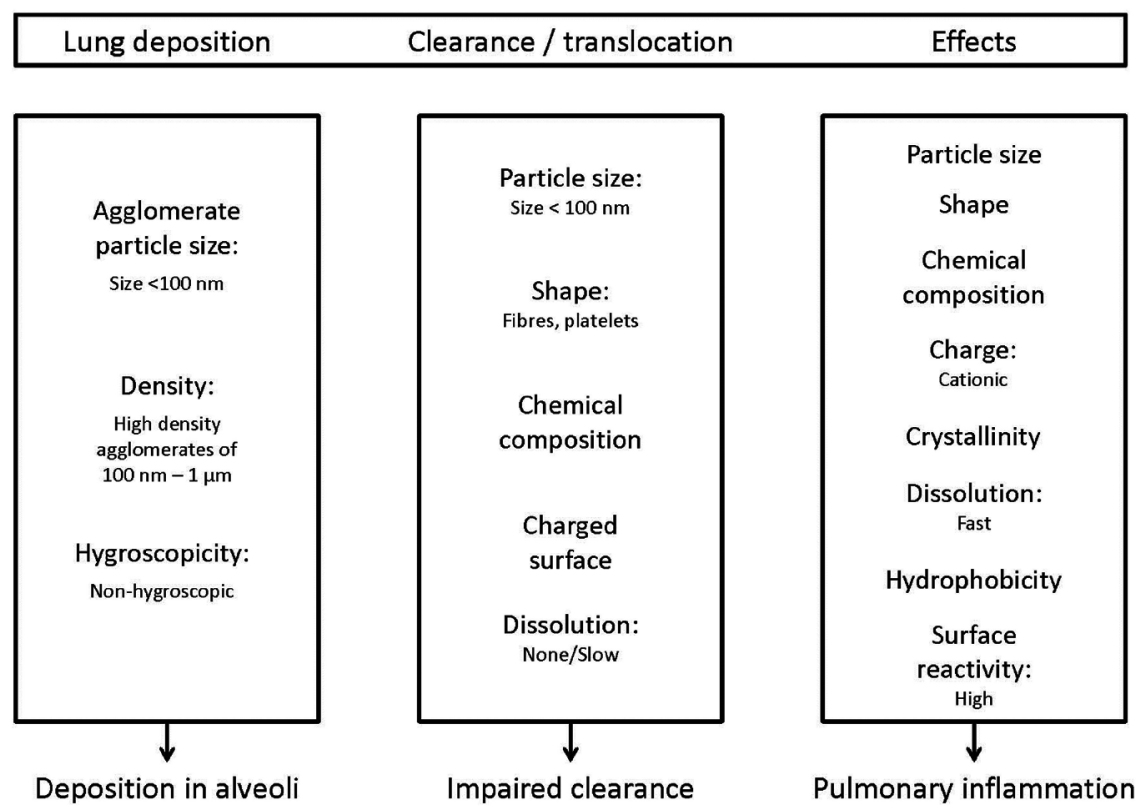

Figure 3. Nanomaterial characteristics resulting in increased lung deposition (in alveoli), impaired clearance rate, and the induction of pulmonary inflammation. The figure shows the physicochemical characteristics of nanoparticles that result in 1) Increased lung deposition in the alveoli (left panel): Nanoparticles with a primary/agglomerate size of $<100 \mathrm{~nm}$, or an agglomerate size between $100 \mathrm{~nm}$ and $1 \mu \mathrm{m}$ with a high density, will deposit efficiently in the alveolar region. Non-hygroscopic nanoparticles will not grow in size by water uptake, resulting in a higher chance to reach the alveoli. 2) Impaired clearance rate (middle panel): Particles/agglomerates of $<100 \mathrm{~nm}$ are less efficiently phagocytised, nano-fibres and platelets are less efficiently cleared compared to spheres, chemical composition influences clearance rate, charged nanoparticles attract proteins and reduce their clearance, and none or slowly dissolving nanoparticles are less efficiently cleared compared to fast dissolving nanoparticles. 3) The induction of pulmonary inflammation (right panel): After deposition of nanoparticles in the alveoli, the shown characteristics all influence the induction of pulmonary inflammation. Cationic particles are easily taken up by cells, fast dissolving nanoparticles can release toxic ions, and nanoparticles with a high surface reactivity can damage the lungs.

Besides particle size, other nanoparticle characteristics influence deposition, clearance, and induction of pulmonary inflammation (figure 3). All these characteristics affect different stages of the events leading to pulmonary inflammation; no single characteristic can be identified as the most important in the induction of pulmonary inflammation by nanomaterials. Measuring the surface reactivity of nanoparticles might be the best way to predict the toxicity of nanoparticles because it combines information on the effects of the chemical composition, shape, size, dissolution rate, and surface area of the particles (Knaapen et al., 2004, Moller et al., 2010). A nanoparticle's surface 
reactivity depends on the exposure medium, in which proteins or other macromolecules might attach to the surface of the nanoparticles. As nanomaterials can differ in all physicochemical characteristics, surface reactivity should be measured separately for each type of nanomaterial in the appropriate medium. Since there is no validated in vitro assay available to test all types of nanomaterials, a high-throughput in vitro assay should be developed and validated to determine the intracellular production of Ros after nanoparticle exposure. Still, animal studies are needed to validate the assays, as surface reactivity in vitro may differ from surface reactivity in vivo, when nanoparticles react with macromolecules in the body and where cellular concentrations are much lower.

If surface reactivity indeed turns out to be the best predictor for the toxicity of nanomaterials, it could be used to categorize nanomaterials into hazard groups. This might help reduce animal testing and speed up risk-assessment procedures, which are currently approached on a case-by-case basis. In addition, if a universal unit of surface reactivity can be defined, it can be included in the dose metric of nanomaterials. Still, while surface reactivity and the ability of nanomaterials to produce oxidative stress appear to be important predictors for toxicity in the lung, the effects of other nanomaterial characteristics should not be ruled out completely. Not all nanomaterial characteristics have been investigated to the same extent, and new types of nanomaterials are continuously being developed, taking on more complex forms that may be associated with new mechanisms of toxicity. A close collaboration between developers of nanomaterials and nanotoxicologists is necessary for the development of new nanomaterials with promising benefits and low risks.

Although very little is known on the exact clearance rates and retention of nanoparticles in the lungs, there is concern that non-soluble nanoparticles will be retained in the lungs and secondary organs for years. The phagocytosis of nanoparticles by macrophages is slower compared to microparticles (Bakand et al., 20I2, Muhlfeld et al., 2008a, Geiser et al., 2008), and nanoparticles deposit deeper in the lungs, where the clearance is slower due to increased pathway length and decreased mucous velocity (Geiser and Kreyling, 20 I0). A low-level but longer-lasting or repeated exposure might enable delayed or slowly developing pulmonary inflammation that is not resolved over time. Compared to larger particles, increased retention of nanoparticles may damage the lungs or may result in the translocation to secondary organs. It is important to note that in general, translocation to the systemic circulation is very low, below $0.5 \%$ of the exposure concentration (Geraets et al., 20I 2, Kreyling et al., 2002, Kreyling et al., 2009, Sadauskas et al., 2009). It is not clear if there is a cut-off point in particle size beyond which particles can no longer translocate; under overload conditions, even larger particles might translocate.

Long-term animal studies are very expensive; therefore, there is a great need for alternative methods or new ways of combining information to predict long-term accumulation and effects of nanomaterials in the body. Even for short-term effects, not all characteristics can be studied in detail in animal studies, because the variation 
in nanomaterials is just too large. Therefore, alternative methods need to be developed for high-throughput screening of nanomaterials. Some co-culture in vitro systems have been developed that mimic the lung barrier (Rothen-Rutishauser et al., 2005, Huh et al., 20 I O, Hermanns et al., 2004, Klein et al., 20 I I, Klein et al., 2013) and hold promise for testing nanomaterials with diverse characteristics, although parallel in vivo studies to demonstrate their predictive value are often lacking. Finally, human epidemiological studies conducted in the workplace, for example, can provide useful information on the effects of nanomaterials after inhalation.

Box 2. Recommendations to find the physicochemical characteristics of nanomaterials that affect pulmonary inflammation.

\section{Recommendations}

- The impact of a nanomaterial characteristic should be systematically tested in different experimental setups by using a number of different nanomaterials, varying only in a single physicochemical characteristic e.g. surface reactivity, and analysed by controlling for other variables such as size and chemistry.

- Surface reactivity should be investigated as a valuable predictor for a nanomaterial's potential to induce pulmonary inflammation.

- The data from studies that systematically tested a single nanomaterial characteristic can be combined into a large data set to carry out multivariate analysis to determine potential combinations of characteristics that are important in affecting pulmonary inflammation.

- Agglomerate particle size should be measured as closely as possible to the site of exposure in animal studies or in occupational exposure settings in order to accurately predict the lung deposition by the particle dosimetry models such as the MPPD model.

- Nanomaterial characteristics should be measured in the appropriate medium, because reaction with macromolecules in biological fluids will change nanoparticle characteristics and thereby their cellular uptake and effect.

- There is a need to generate data on biodistribution and accumulation of nanomaterials upon long-term exposure, as well as the induction of toxic effects, to assess which of the physicochemical properties have the largest influence on delayed or chronic pulmonary inflammation. 
Since no single nanoparticle property can be identified as the most important in the induction of pulmonary inflammation, we have some recommendations for future research (Box 2). The ultimate goal is to categorize nanomaterials according to their characteristics, which would be of immense value for the risk assessment of these wideranging and fast-developing products.

\section{OBJECTIVES AND OUTLINE OF THE THESIS}

Nanomaterials can vary in aspects such as size, shape, charge, crystallinity, chemical composition, and dissolution rate. Many physicochemical characteristics of nanoparticles affect their lung deposition, clearance, and pulmonary response that, in combination, ultimately determine whether pulmonary inflammation will occur and to what extent. With all these characteristics affecting different stages of the events leading to pulmonary inflammation, no unifying dose metric could be identified to describe pulmonary inflammation for all nanomaterials. Therefore, the main goal of this thesis is:

'to understand, assess, and implement in risk assessment the dose metric(s) that is (are) most suited for modelling the dose-effect relationship of nanomaterials for human health'

\section{To achieve this goal, the following objectives are formulated:}

I. Determination of the dose metric which provides the best descriptor for dose-response relationships of nanomaterials after inhalation exposure

In vivo inhalation studies are performed to determine the most suitable dose descriptor for nanomaterials after inhalation. For these studies, the relationship between external exposure and the internal dose is of importance as the dose at the site of action determines whether adverse effects occur. Therefore, we first describe the possibilities and limitations of available techniques to detect nanomaterials in biodistribution studies (chapter 2). Second, a pilot study with two different sizes of silver nanoparticles was performed to confirm the hypothesis that smaller nanoparticles induce more pronounced effects compared to larger particles after inhalation (chapter 3). Next, a larger study with four different sizes of silver nanoparticles was performed to obtain dose-response relationships that were analysed based on different dose metrics (chapter 4).

2. Evaluation of in vitro models to determine suitable dose metrics of nanomaterials and their bioavailability after inhalation exposure

Although animal studies provide unique information on the distribution and effects of nanomaterials, the use of animals is ethically debatable and should be reduced. In addition, giving the large variety in nanomaterials, it is impossible and economically not feasible to test all of them using in vivo studies. Therefore, many in vitro models have been developed to test the distribution and effects of nanomaterials. First, we evaluated the progress and future of in vitro models to study the translocation of nanomaterials (chapter 5). Second, 
we evaluated the use of an in vitro lung barrier to study the translocation of fluorescent polystyrene nanoparticles (chapter 6). Third, we used the in vitro lung barrier model under submerged conditions to determine the dose metric to describe the effect of silver nanoparticles in vitro and compared this to the in vivo results (chapter 7). Finally, we exposed the in vitro lung model to silver nanoparticles at the air-liquid interface to mimic more realistically inhalation exposure compared to submerged exposure (chapter 8).

3. Implementation of dose metrics in the risk assessment of nanomaterials after inhalation exposure, including a testing strategy

The main goal of this thesis is to determine and implement the most suitable dose metric to describe the dose-effect relationship of nanoparticles after inhalation. The applicability domain of the selected dose metrics will be discussed, i.e. whether it is applicable for different biological responses, different particles and external or internal doses (chapter 9). In addition, a testing strategy will be presented to assess the acute effects of nanomaterials after inhalation in which the use of dose metrics and in vitro methods play a central role. 
Adapted from: Applications of inductively coupled plasma mass spectrometry (ICP-MS) in biodistribution studies of gold, silver and titanium dioxide nanomaterials

Petra Krystek ${ }^{\mathrm{I},}{ }^{,}$, Hedwig M. Braakhuis ${ }^{2,3}$, Margriet V.D.Z. Park ${ }^{3}$, Wim H. de Jong ${ }^{3}$

Encyclopedia of Analytical Chemistry 2013; John Wiley \& Sons

${ }^{\text {I }}$ Philips Innovation Services, High Tech Campus I I, 5656 AE Eindhoven, the Netherlands

${ }^{2}$ Department of Toxicogenomics, Maastricht University, Po Box 6I6, 6200 MD Maastricht, the Netherlands

${ }^{3}$ National Institute for Public Health and the Environment (RIVM), PO Box I, 3720 BA

Bilthoven, the Netherlands 


\section{Applications of inductively coupled plasma mass spectrometry (ICP-MS) in biodistribution studies}




\section{ABSTRACT}

The biodistribution of engineered inorganic nanomaterials with size characteristics of one, two or three dimensions smaller than $100 \mathrm{~nm}$ is a fast growing analytical and toxicological research field. Exposure to NPs is possible via inhalation, injection, ingestion and skin contact. The distribution of NPs in the body is affected by their chemical/ elemental composition, size and size distribution, shape, coatings, surface properties and their stability under the selected circumstances. The evaluation of the presence of nanoparticles to determine their tissue distribution is not easy. Nanoparticle presence can be identified by electron microscopy but this is a time and labour consuming technique. So, instead of determining the presence of the nanoparticles themselves distribution studies can use elemental analysis by inductively coupled plasma mass spectrometry (ICP-MS) as indication for the tissue distribution of nanoparticles. In the field of analytical research, the suitable application of ICP-MS is crucial and discussed in detail. Other relevant analytical techniques are presented too.

\section{GENERAL INTRODUCTION}

The biodistribution of engineered inorganic nanomaterials is a fast growing analytical and toxicological research field. Nano-objects are defined as materials with at least one dimension in the nanoscale range below $100 \mathrm{~nm}$. Furthermore, the nano-objects are distinguished into nanoparticles (NPS) with three, nanofibres with two and nanoplates with one dimension in the nanoscale range (< IOO nm) (Krystek et al., 20 I I ). Exposure to NPS is possible via inhalation, injection, ingestion and skin contact. The distribution of NPS in the body is affected by their chemical/elemental composition, size and size distribution, shape, coatings, surface properties and their stability under the selected circumstances. For risk assessment purposes, information is needed on the actual dosage of NPs at the site of toxicity. Biodistribution studies provide information on the uptake and clearance of NPS in the body after exposure; they give insight in which organs and tissues the NPs accumulate. This information is important to determine which organs are at risk for suffering adverse effects from the NPS.

From an analytical point of view, inductively coupled plasma mass spectrometry (ICPMs) plays an important role in gaining knowledge on the distribution of NPs. Nowadays, the main application is the total determination of the consisting elements from the NPS of interest in various organ and body fluid samples (Krystek, 20 I2). Next to this, ICPMs has the potential to be applied as a technique for the detection of single particles (sp) and as an element-specific detector after the separation of NPs by size. For the size separation, systems based on the field flow fractionation (FFF) principle might be the technique of choice in the future. Additionally, electron microscopy (EM) techniques are used to gather more information about the more local particle distribution in tissues. This review gives an overview of the use of ICP-MS in distribution studies. 


\section{SAMPLING OF THE TISSUES AND BODY FLUIDS}

NPS can be administered to animals by inhalation, orally, intravenously, or via the skin. After exposure, the amount of NPS in the tissues and organs can be quantified by ICP-MS. For quantification of NPS in tissue samples by ICP-MS, the animals need to be sacrificed. Digests of tissue sample are measured by ICP-MS to determine the concentration. These raw data are used for calculating the amount of NPs per gram of tissue. Therefore, it is important that the organs and tissues of interest are isolated as a whole, with minimal fat around it, and carefully weighed. Organs that are mostly analysed in biodistribution studies with NPs are the liver, spleen, kidneys, lungs, heart, brain, blood, testis/ovaries and thymus (Khlebtsov and Dykman, 20 I I, De Jong et al., 2008, Lankveld et al., 20 I , Lankveld et al., 20 I0, Wang et al., 2007). For ICP-MS analysis, only a small amount of tissue is needed; 0.5 grams per organ or tissue is sufficient. To prevent selective choice/ collection of tissue samples, the organs must be carefully homogenized before taking a tissue sample. If needed, the samples can be stored at a temperature below $-\mathrm{I} 8^{\circ} \mathrm{C}$ before analysis.

\section{DISSOLUTION OF TISSUES OR BODY FLUIDS AND DILUTION OF BODY FLUIDS}

Within biodistribution studies, often larger series of samples must be analysed. Therefore, it is crucial to pay sufficient attention to developing a method which is not very timeconsuming during the performance or during pre-treatment steps, like cleaning a lot of lab ware articles (e.g. vials). In general, acid digestion is applied for the dissolution of tissues and particle containing body fluids (Krystek, 20I2, De Jong et al., 2008, Lankveld et al., 20I I, Lankveld et al., 20I0). Therefore a microwave system or open digestion procedures by heating block systems can be used. However, a closed digestion in a microwave lowers the risk of losses and/or contamination in comparison to open digestion procedures.

Depending on the analytical or distributional question of interest, different acid mixtures might be applied. Au NPs in tissues are generally dissolved in aqua regia while a heating block system for open digestions (Krystek, 20I2) is used. Comparable procedures for Au NPS in tissues are extended and the residual aqua regia is evaporated by heating at $126^{\circ} \mathrm{C}$ in an oil bath. The residue is then re-dissolved in $\mathrm{Io} \mathrm{mL}$ of $0.05 \mathrm{M}$ hydrochloric acid (Sonavane et al., 2008). Closed digestion by microwave is possible too (Krystek et al., 20 I I, Sadauskas et al., 2009). In this case, $5 \mathrm{~mL}$ nitric acid, $0.5 \mathrm{~mL}$ hydrogen peroxide are added and the samples were digestion for 60 minutes at 600 Watt, then I $\mathrm{mL}$ hydrochloric acid is added and additionally io minutes at 6ooWatt (Khlebtsov and Dykman, 20 I I). Ag NPs in tissues are often dissolved under comparable circumstances by nitric acid. This is the case when the general distribution of the engineered Ag NPs is the question of interest (Khlebtsov and Dykman, 20 I I). Nevertheless, in biological media Ag might also form other Ag compounds like Ag chloride or Ag sulphide (Huda et al., 
20I0). As long as Ag is not (totally) stabile under the various biological circumstances in the body's process, these compounds do not dissolve in nitric acid.

In several cases also other solvents are used to dissolve Ag NPs in tissues by microwave digestion. The settings are $250^{\circ} \mathrm{C}, 70$ bar for 90 minutes while nitric acid (van der Zande et al., 20I2) or aqua regia (Krystek and De Jong, 20I2) are used as solvents. Nevertheless, it is also found that for the digestion of the Ag NP suspension other digestion media like a mixture of concentrated hydrochloric and sulphuric acid (3:I) can be used by running the same microwave digestion procedure (van der Zande et al., 20 I 2). Normally, for urine samples a simple I: Io dilution with diluted acid is sufficient (Krystek and Ritsema, 2002, Krystek and Ritsema, 2009b). For blood samples, in several cases a I:20 dilution might be sufficient (De Boer et al., 2004). Nevertheless, as long as traces of non-dissolved NPS are of interest, the more intense microwave or heating block digestions are preferred.

For the dissolution of $\mathrm{TiO}_{2}$ NPs various acid mixtures are known. The digestion is possible with concentrated sulphuric acid. These samples are heated at $65^{\circ} \mathrm{C}$ in polypropylene or $250^{\circ} \mathrm{C}$ (borosilicate glass) for 0.5 to $\mathrm{I} 2$ hours until a clear solution was achieved (Lomer et al., 2000). Within another procedure a mixture of concentrated nitric acid and hydrofluoric acid (3:I) is used. The samples are heated slowly to $90^{\circ} \mathrm{C}$ in polypropylene tubes and are kept at this temperature until the biological sample material is totally dissolved. It is important to remember that glass or quartz tubes cannot be used due to the use of hydrofluoric acid which dissolves glass (Sarmiento-Gonzalez et al., 2009, www.nanogenotox.com, 20I2). Prior measurement these samples should be sufficiently diluted. Otherwise, for the ICP-MS a special sample introduction system which is resistant against hydrofluoric acid must be used.

\section{MEASUREMENT BY ICP-MS}

\section{Selected isotopes and possible interferences}

All digests of the biological samples as well as control or reference material and chemical blanks are measured by ICP-Ms (Montaser, I998, Jakubowski et al., I998). By using a collision cell (CC) or dynamic reaction cell (DRC) in combination with quadrupol (Q)ICP-MS or the high resolution (HR)-ICP-MS, an interference-free measurement should be possible (Khlebtsov and Dykman, 20I I). Because Au is a mono-isotopic element, the measurements must be carried out on ${ }^{197} \mathrm{Au}$. Fortunately, there is no frequently occurring interference on $\mathrm{m} / \mathrm{z}=\mathrm{I} 97$. Nevertheless, only unusual interference like ${ }^{157} \mathrm{Gd}^{40} \mathrm{Ar}^{+}$or ${ }^{18}{ }^{1} \mathrm{Ta}^{16} \mathrm{O}^{+}$(Audi and Wapstra, I995) might occur. Ag consists of two isotopes with the following abundances: ${ }^{107} \mathrm{Ag}(5 \mathrm{I} .84 \%)$ and ${ }^{109} \mathrm{Ag}(48.16 \%)$. On ${ }^{107} \mathrm{Ag}^{+}$possible interferences are: ${ }^{67} \mathrm{Zn}^{40} \mathrm{Ar}^{+},{ }^{71} \mathrm{Ga}^{36} \mathrm{Ar}^{+},{ }^{91} \mathrm{Zr}^{16} \mathrm{O}^{+}$and ${ }^{89} \mathrm{Y}^{18} \mathrm{O}^{+}$(Audi and Wapstra, I995). If HR-ICP-MS is used only ${ }^{89} \mathrm{Y}^{18} \mathrm{O}^{+}$still leads to interferences, all other interferences can 
be separated by using a sufficient high resolution mode. On ${ }^{109} \mathrm{Ag}^{+}$only ${ }^{69} \mathrm{Ga}^{40} \mathrm{Ar}^{+}$or ${ }_{93} \mathrm{Nb}^{16} \mathrm{O}^{+}$(Audi and Wapstra, I 995) might interfere. However, by using HR-ICP-MS the ${ }^{69} \mathrm{Ga}^{40} \mathrm{Ar}^{+}$may be overcome. Ti consist of five isotopes with the following abundances ${ }^{46} \mathrm{Ti}(8.00 \%),{ }^{47} \mathrm{Ti}(7.30 \%),{ }^{48} \mathrm{Ca}(73.80 \%),{ }^{49} \mathrm{Ti}(5.50 \%)$ and ${ }^{50} \mathrm{Ti}(5.40 \%)$ (Audi and Wapstra, I995). At $\mathrm{m} / \mathrm{z}=46$ and $\mathrm{m} / \mathrm{z}=48$ isobaric interferences from Ca exists. Also $\mathrm{m} /$ $\mathrm{z}=50$ is isobaric interfered by $\mathrm{Cr}$ and $\mathrm{V}$. Therefore, only $\mathrm{m} / \mathrm{z}=47$ and $\mathrm{m} / \mathrm{z}=49$ can be used but also here possible polyatomic interferences must be taken into account and an advanced measurement mode of HR-, CC- or DRC-ICP-MS must be used. On ${ }^{47} \mathrm{Ti}^{+}$ the most common interferences are ${ }^{31} \mathrm{P}^{16} \mathrm{O}^{+},{ }^{11} \mathrm{~B}^{36} \mathrm{Ar}^{+}$and ${ }^{15} \mathrm{~N}^{16} \mathrm{O}_{2}^{+}$. On ${ }^{49} \mathrm{Ti}^{+}{ }^{33} \mathrm{~S}^{16} \mathrm{O}^{+}$, ${ }^{31} \mathrm{P}^{18} \mathrm{O}^{+},{ }^{9} \mathrm{Be}^{40} \mathrm{Ar}^{+}$and ${ }^{14} \mathrm{~N}^{18} \mathrm{O}^{16} \mathrm{O}^{1} \mathrm{H}^{+}$might interfere (www.nanogenotox.com, 2012, Linglan et al., 2009).

\section{Quantification by external calibration}

With a state-of-the-art approach, external calibration will be always carried out by internal standard correction. For ${ }^{197} \mathrm{Au}^{+}$typically ${ }^{103} \mathrm{Rh}^{+}$(Krystek et al., 20 I I , Cho et al., 2009) ${ }^{20}{ }^{205} \mathrm{Tl}^{+}$(Krystek, 20I 2) are used as internal standard. ${ }^{107} \mathrm{Ag}^{+}$resp. ${ }^{109} \mathrm{Ag}^{+}$can be corrected by using ${ }^{103} \mathrm{Rh}^{+}$(Khlebtsov and Dykman, $\left.20 \mathrm{I} \mathrm{I}\right)$, for ${ }^{47} \mathrm{Ti}^{+}$or ${ }^{49} \mathrm{Ti}^{+{ }^{115}} \mathrm{In}^{+}$can be used (Sadauskas et al., 2007).

\section{Setup and relevant quality control experiments}

General checks like the blank of the used chemicals must be carried out. If there is a significant contribution of the elements of interest in the chemicals, it is self-evident that a correction must be carried out. It is also recommended to perform several independent duplicates. Furthermore, the information will be more significant if the duplicates are analysed in independent sequences. If a detailed method validation is required, the determination of the most relevant performance characteristics (like the limit of detection, repeatability, reproducibility, accuracy and measurement uncertainty) should be carried out in a state-of-the-art manner. One general example about the method validation is given in (Krystek and Ritsema, 2009a).

For accuracy control, it is essential to analyse a (certified) reference material (RM) within the same sequence. However, there are just a few suitable RMs for NPs in biomedical research commercially available, e.g. from the National Institute of Standards (NIST); e.g. Au spheres nominally io nm (NIST RM8OIO), $30 \mathrm{~nm}$ (NIST RM8OII) and $60 \mathrm{~nm}$ (NIST RM8OI2). For the other elements, general biological reference materials from a comparable concentration level of the total elemental concentration of interest should be at least used; e.g. products like trace elements in whole blood or urine and freezedried organs like e.g. liver (Krystek et al., 20 I I). 


\section{ADDITIONAL APPLIED TECHNIQUES FOR THE LOCALISATION OF NPS IN ORGANS OR TISSUES}

ICP-MS is a suitable technique to quantify the amount of NPS in organs or tissues. However, the technique only provides quantitative information about the presence of a particular chemical composition in the organs as a whole. There is no information about the state of the particles, i.e. whether they have aggregated/agglomerated or changed size or shape. In addition, there is no information about the more local distribution of NPS within the organs and tissues. Besides information on organs as a whole, information on the presence of NPs within specific cell types of the organs is valuable for the risk assessment of nanomaterials. Information on what types of cells are involved in the uptake of nanomaterials provides information on which processes might be induced by exposure to nanomaterials. The cell types that are mostly involved in the uptake of nanomaterials can be investigated in more detail in in vitro studies to determine the mechanism underlying the effects of nanomaterials.

There are various techniques available to localize NPs in tissues and organs; histology, autometallography, scanning electron microscopy (SEM), and TEM techniques are in common use (Krystek, 20I2). Histology and autometallography are light microscopic (LM) techniques and can be used to localize larger particles or aggregates of smaller particles in tissues. For histology, cells and tissues are fixed in formaldehyde and embedded in paraffin. Paraffin coupes can be stained with different staining protocols to colour cellular structures; for example, to determine tissue damage caused by nanomaterials. SEM and TEM are electron microscopy techniques. Compared to LM techniques, the resolution of EM techniques is better up to I nm resolution; structures at the nanoscale can be distinguished from another. These techniques require laborious tissue preparation before imaging. The electron beam of EM techniques requires a vacuum; therefore, samples should be completely fixed and dehydrated. SEM can use back-scattered electrons (BSE) that reflect a flat $2 \mathrm{D}$ surface within the tissue sample or secondary electrons (SE) that reflect the 3D surface of the tissue sample. TEM is the preferred technique to localize nanomaterials in tissues as the resolution is better compared to SEM and enables imaging of individual nanomaterials. Depending on the electron density of the nanomaterials and the concentration of the nanomaterials in the tissue, the NPs can be detected. For example, Au and Ag NPs are electron dense and can be easily recognized within the tissues, especially because $\mathrm{Au}$ and $\mathrm{Ag}$ are normally not present in human or animal tissues. In addition, the shape of the particles can also help to identify the nanomaterials. Spherical shapes are easier to recognize than irregular shaped particles. Also, particles with a small size distribution are easier to identify than particles with a wide size distribution. For less electron-dense NPs it can be more difficult to distinguish them from tissue organelles such as glycogen spheres. To verify if electron dense shapes in the tissue samples are the NPS that you are looking for, SEM or TEM can be combined with EDX to obtain elemental data. This technique measures the element-specific X-rays of the area of interest and identifies which elements are present in the tissue. 
A disadvantage of EM is that the technique provides information on only a minor part of the whole tissue. It is not possible to visualize the whole tissue of interest by EM; you need to know where to look. This is also why quantification of NPs using EM alone is complex if not impossible. Therefore, the combination of ICP-MS and EM is a valuable tool to first identify which organs or tissues contain the most nanomaterials and then localize the NPS at ultra-structural level using EM. Finally, EDX can be used to verify the results.

\section{FUTURE CHALLENGES OF ICP-MS IN BIODISTRIBUTION STUDIES}

Quantitative methods such as ICP-MS are used to study the biodistribution of nanomaterials. ICP-MS can detect the elements of nanomaterials and the evaluation of their biodistribution quantitatively. However, ICP-MS cannot distinguish between elements that are inherent to the nanomaterials and those that are cleaved or released from them. But, unlike ICP-MS, TEM can detect the presence of nanomaterials and identify their location within tissues and cells. To study the biodistribution of nanomaterials quantitatively methods such as ICP-MS should be used, as for e.g. TEM several hundred if sections might be necessary (Tsutsumi and Yoshioka, $20 \mathrm{I}$ I). There are various techniques and approaches which allow direct analysis of NPs by getting not only information on chemical composition but also size simultaneously (Ulrich et al., 2012). The particle size, which includes size distribution and aggregation state, is arguably one of the most important properties to quantify biodistribution of NPs and when conducting toxicity tests (Pace et al., 20I2). In relation to ICP-MS, other approaches for solution / diluted suspensions show potential to be applied:

a) Single particle (sp) mode ICP-MS

b) Hyphenated techniques with ICP-MS

\section{Single particle (sp) mode ICP-MS}

Depending on the element, the minimum detectable nanoparticle size in sp-ICP-Ms mode ranges from 20 to $30 \mathrm{~nm}$; e.g. a detection limit of $\mathrm{I} 8 \mathrm{~nm}$ for $\mathrm{Ag}$ NPs was recently reported (Laborda et al., 20II). The minimum detectable size limit is given by the relatively small number of atoms present in a single nanoparticle and the minimum element mass required to achieve ICP-MS signals from the single particle event distinguishable from the background (Gschwind et al., 20II). On the other hand, the upper size for direct particle analysis in ICP-MS is mainly given by the sample introduction system, especially by the cut-off of the used spray chamber ranging typically between I.4 and $2 \mu \mathrm{m}$. The chosen setting of the plasma power is of great importance for achieving a complete ionisation of particles up to the micrometer size (Krystek et al., 20 I I, Ulrich et al., 20 I 2). Beyond method sensitivity limitations, many current techniques have been shown to be well suited to characterise polydisperse systems that contain aggregates 
(Pace et al., 20I2). sp-ICP-MS is an emerging analytical technique that has the potential of addressing some of the major challenges faced by investigators when analyzing for particle size within toxicity tests. Both particle number concentration and particle size can be simultaneously determined by using sp-ICP-Ms (Degueldre et al., 2006a, Degueldre et al., 2006b, Mitrano et al., 2012).

Despite all these advantages, the ability of use sp-ICP-MS to analyse environmental samples still faces many hurdles. For instance, given significant quantities of chloride are present in the sample, the appearance of a second peak in the particle size distribution is hypothesized to be $\mathrm{AgCl}$ precipitates forming in the media following dissolution of the Ag NPs (Pace et al., 20I2). Due to the similar main composition of biological matrices this information is also relevant for samples from biodistribution studies. Especially by the mentioned approaches the stability of the NPs in the related biological matrix is relevant. The colloidal stability and the agglomeration behaviour depend on the nanoparticle-type, the coating or functionalization agent and on the surrounding media. The stability of charge stabilized NPs is especially sensitive to changes of $\mathrm{pH}$ or ionic strength and sometimes even to dilution. Although the stability of sterically stabilized NPs is typically less affected by the mentioned factors, agglomeration can still occur (Ulrich et al., 20 I2).

However, while sp-ICP-MS offers some selectivity, a major disadvantage to sp-ICP-MS at present is that it is not able to distinguish between particles with the different elemental composition. Due to the current limitations of sp-ICP-Ms, where only one isotope can be monitored at a time, it was not possible to differentiate between these two particle types. Experiments about the determination of Ag NPs in tissues and gastrointestinal content by sp-ICP-MS are reported. At first the biological matrix is enzymatic a digested by adding a digestion buffer of $\mathrm{I}$ o $\mathrm{mM}$ Tris, I\% Triton X-ıoo, and I mM calcium acetate; $\mathrm{pH}$ 9.5. By vortexing the samples and due to the addition of proteinase $\mathrm{K}$ $\left(32 \mathrm{U} / \mathrm{mL}\right.$ ) the samples were digested at $55^{\circ} \mathrm{C}$ (van der Zande et al., 20 I2). Typically settings of the sp mode for the two $\mathrm{Ag}$ isotopes $\mathrm{m} / \mathrm{z}=107$ and $\mathrm{I} 09$ are the time-resolved analysis with a dwell time of $3 \mathrm{~ms}$ and an acquisition time of $60 \mathrm{~s}$. The particle sizes are calculated from the measured $\mathrm{Ag}$ masses by assuming a spherical shape of the particles. Also in this study a lower size detection limit of $20 \mathrm{~nm}$ was confirmed (van der Zande et al., 20I2). In the future, approaches of single particle mode in combination with fast scanning options might help to deliver multiple-elemental information about the species composition of inorganic NPS.

\section{Hyphenated techniques with ICP-MS}

In the general approach regarding to the analysis of NPS, ICP-MS is also used as an element specific detector after applying a suitable size separation technique for NPS. In this case, two or more instruments are often only on-line coupled. Hydrodynamic chromatography (HDC) allows to achieve size fractionation of colloidal particles in 
the range of a few hundred Angstrom to a few microns in diameter; HDC-ICP-MS is employed for the characterization of Ag and Au NPs in aqueous matrices (Tiede et al., 20I0). Studies of the selected inorganic NPs in biological matrices are not known to the authors of this paper.

The most favourite technique for the separation of NPs is the field flow fractionation technique (FFF) which is applicable for liquid samples (Giddings, I973, Wahlund and Giddings, 1987). FFF is often coupled to several detectors like UV-detector, detectors for the determination of the particle size by dynamic light scattering (DLS) or multiple angle light scattering (MALS) and ICP-MS for the elemental composition. In the field of FFF, different separation principles are available based on thermal, symmetric or asymmetric flow, electrostatic force, sedimentation, gravitational, or magnetic field force as well as their combinations (see also Table I) (Krystek et al., 20I I). The two mainly used FFF principles are the sedimentation (Sed) and the asymmetric flow field flow fractionation (AF4). SedffF has been used e.g. for analysis of inorganic colloids, pigments, viruses, cells, liposomes as well as DNA or RNA (Kirkland et al., I980, Chianea et al., 2000). AF4 is the most established fFF technique in bio-analysis e.g. for the analysis of polysaccharides, proteins and protein complexes, viruses, nano- and microsized beads or sub-cellular units (Krystek et al., 20I I, Silveira et al., 2005, Liu et al., 2006, Ratanathanawong et al., 2006, Lee et al., 200I). Also for the characterization of Au NPS in a complex biological matrix, AF4 with various detectors is applied (Krystek et al., 20I2). Examples for hyphenated systems of FFF with mass spectrometric detection are published; e.g. the bacterial analysis method for $P$. putida and E. Coli by coupling the flow field-flow fractionation (flow FFF) separation technique with detection by matrix-assisted laser desorption/ionization time-of-flight mass spectrometry (MALDI-TOF-MS) (Lee et al., 2003). Hollow-fiber flow field-flow fractionation ( $\left.\mathrm{HF}_{4}\right)$ is applied to untreated, whole human blood serum and the HF4 is on-line coupled to MALDI-TOF-MS of serum fractions shows mass signals in the $<30,000 \mathrm{M}(\mathrm{r})$ range (Zattoni et al., 2008). The development of applications with FFF-ICP-MS is coming up slowly. One of the first examples in the biomedical field is the speciation analysis of plasma proteins by asymmetricflow field flow fractionation (AF4)-ICP-MS (Herault et al., 20I2). However, about the application of FFF-ICP-MS for biodistribution studies of inorganic NPs as described above, articles were not be found in the literature up to now.

\section{CONCLUSION}

The use of ICP-MS in biodistribution studies gives a lot of valuable, quantitative information for the risk assessment of inorganic nanomaterials. With the classical approach of digestion and the determination of the total concentration of the element of interest, larger series of tissue samples can be analysed within a reasonable period of time, allowing for sufficient information about the distribution in various organs and body fluids of interest. New challenges and possibilities lie ahead for the application of 
ICP-MS in biodistribution studies, e.g. the use of sp-ICP-MS or hyphenated techniques like FFF-ICP-MS. These techniques show more selectivity for nanoparticles, but must be further developed for routine application in this field. Additional analytical challenges involve the analysis of multi-element nanomaterials like CdSe or silica-coated silver NPs.

Table 1. Overview about fFF techniques (Krystek et al., 2011)

\begin{tabular}{|c|c|c|c|c|c|}
\hline $\begin{array}{l}\text { Separation } \\
\text { Force }\end{array}$ & Types & Abbreviation & Size range & Application fields & Adapted detectors \\
\hline Cross flow & $\begin{array}{l}\text { Symmetrical } \\
\text { flow FFF }\end{array}$ & SyF4 & $1 \mathrm{~nm}-1 \mu \mathrm{m}$ & $\begin{array}{l}\text { - } \text { biopolymers e.g. } \\
\text { polysaccharides and } \\
\text { proteins } \\
\text { - } \text { DNA } \\
\text { - Cells } \\
\text { - Virus }\end{array}$ & $\begin{array}{l}\text { UV, FLD, FC, soft- } \\
\text { impact mass } \\
\text { spectrometry } \\
\text { MALLS, FLD }\end{array}$ \\
\hline Cross flow & $\begin{array}{l}\text { Asymmetrical } \\
\text { flow FFF }\end{array}$ & AF4 & $1 \mathrm{~nm}-1 \mu \mathrm{m}$ & $\begin{array}{ll}\text { - } & \text { Inorganic colloids and } \\
\text { - } & \text { Pigs } \\
\text { - } & \text { Inorganic polymers } \\
\text { - } & \text { Lattice polymers } \\
\text { - } & \text { Proteins } \\
\text { - } & \text { DNA, RNA } \\
\text { - } & \text { Cells } \\
\text { - } & \text { Virus }\end{array}$ & $\begin{array}{l}\text { UV, FLD, MALLS, } \\
\text { DLS, RI, ICP-OES, } \\
\text { ICP-MS }\end{array}$ \\
\hline Cross flow & $\begin{array}{l}\text { Hollow fiber- } \\
\text { FFF }\end{array}$ & $\begin{array}{l}\text { HF-FLFFF; } \\
\text { HF5 }\end{array}$ & & $\begin{array}{ll}\text { - } & \text { Blood } \\
\text { - } & \text { Cells } \\
\text { - } & \text { Proteins }\end{array}$ & Mass spectrometry \\
\hline Sedimentation & $\begin{array}{l}\text { Sedimentation } \\
\text { FFF }\end{array}$ & SedFFF & $\begin{array}{l}50 \mathrm{~nm} \text { up to } \\
\text { several } \mu \mathrm{m}\end{array}$ & $\begin{array}{ll}\text { - } & \text { Inorganic colloids } \\
\text { - } & \text { Pigments } \\
\text { - } & \text { Virus } \\
\text { - } & \text { Liposoms } \\
\text { - } & \text { DNA, RNA }\end{array}$ & $\begin{array}{l}\text { UV, LS, GFAAS, ICP- } \\
\text { OES, ICP-MS }\end{array}$ \\
\hline Electrostatic & & ElecFFF & & - $\quad$ Proteins & \\
\hline Thermal & & TFFF & & $\begin{array}{ll}\text { - } & \text { Linear polystyrenes } \\
\text { - } & \text { Lypophilic polymers } \\
& \text { Materials of raffinery } \\
\text { industry }\end{array}$ & \\
\hline Gravitation & & GdFFF & & $\begin{array}{ll}\text { - } & \text { Blood, plasma } \\
\text { - } & \text { polystyrene (PS) } \\
\text { microbeads }\end{array}$ & \\
\hline Magnetic & & MagFFF & & $\begin{array}{l}\text { - Magnetic polymer beads } \\
\text { - Magnetic NPs }\end{array}$ & \\
\hline
\end{tabular}



Hedwig M. Braakhuis ${ }^{1,2^{*}}$, Ilse Gosens ${ }^{2}$, Petra Krystek ${ }^{3}$, A. John F. Boere ${ }^{2}$, Flemming R. Cassee $^{2,4}$, Paul H.B. Fokkens ${ }^{2}$, Jan Andries Post ${ }^{5}$, Henk van Loveren ${ }^{1,2}$, Margriet V.D.Z. Park ${ }^{2}$

Particle and Fibre Toxicology 20 I 4 September I7; I I:49

${ }^{\text {I }}$ Department of Toxicogenomics, Maastricht University, po Box 6I6, 6200 MD Maastricht, the Netherlands

${ }^{2}$ National Institute for Public Health and the Environment (RIVM), PO Box I, 3720 BA

Bilthoven, the Netherlands

${ }^{3}$ Philips Innovation Services, High Tech Campus I I, 5656 AE Eindhoven, the Netherlands

${ }^{4}$ Institute of Risk Assessment Sciences, Utrecht University, PO Box 80.163, 3508 TD Utrecht, the Netherlands

'Cellbiology, Biology Department, Faculty of Science, University of Utrecht, po box 80.056, 3508 тв Utrecht, the Netherlands 


\section{Particle size dependent deposition and pulmonary inflammation after short- term inhalation of silver nanoparticles}




\section{ABSTRACT}

Background: Although silver nanoparticles are currently used in more than 400 consumer products, it is not clear to what extent they induce adverse effects after inhalation during production and use. In this study, we determined the lung burden, tissue distribution, and the induction and recovery of adverse effects after short-term inhalation exposure to $\mathrm{I} 5 \mathrm{~nm}$ and $4 \mathrm{IO} \mathrm{nm}$ silver nanoparticles.

Methods: Rats were nose-only exposed to clean air, I $5 \mathrm{~nm}$ silver nanoparticles ( $179 \mu \mathrm{g}$ / $\mathrm{m}^{3}$ ) or 4IO nm silver particles $\left(\mathrm{I} 67 \mu \mathrm{g} / \mathrm{m}^{3}\right) 6$ hours per day, for four consecutive days. Tissue distribution and the induction of pulmonary toxicity were determined at 24 hours and 7 days after exposure and compared with the internal alveolar dose. Presence of silver nanoparticles in lung cells was visualized by transmission electron microscopy (TEM).

Results: Exposure to $15 \mathrm{~nm}$ silver nanoparticles induced moderate pulmonary toxicity compared to the controls, indicated by a 175 -fold increased influx of neutrophils in the lungs, a doubling of cellular damage markers in the lungs, a 5 -fold increase in proinflammatory cytokines, and a I. 5 -fold increase in total glutathione at 24 hours after exposure. All the observed effects disappeared at 7 days after exposure. No effects were observed after exposure to 4IO nm silver particles. The internal alveolar mass dose of the I $5 \mathrm{~nm}$ nanoparticles was 3.5 times higher compared to the 4 IO $\mathrm{nm}$ particles, which equals to a 66,00o times higher particle number. TEM analysis revealed I $5 \mathrm{~nm}$ nanoparticles in vesicles and nuclei of lung cells, which were decreased in size to $<5 \mathrm{~nm}$ at 24 hours after exposure. This demonstrates substantial dissolution of the silver nanoparticles.

Conclusion: The results show a clear size-dependent effect after inhalation of similar mass concentrations of I $5 \mathrm{~nm}$ and 4IO nm silver (nano)particles. This can be partially explained by the difference in the internal alveolar dose between the $15 \mathrm{~nm}$ and $4 \mathrm{IO} \mathrm{nm}$ silver (nano)particles as well as by a difference in the release rate of silver ions.

\section{BACKGROUND}

Nanomaterials are used in a rapidly increasing number of products including consumer products (Nanotechnologies, 20I4). However, there are concerns these nanomaterials might introduce health risks upon occupational and consumer exposure. Although many nanomaterials are produced, handled and present in fluids, aerosolization may occur during energetic processes, such as vortexing, weighing, sonication, mixing and blending (Maynard and Kuempel, 2005). In addition, nanomaterials may be released from their matrix during its use. Therefore, inhalation is considered a relevant route of exposure (Maynard and Kuempel, 2005, Christensen et al., 2010). Currently, silver nanoparticles are the most common nanoparticles mentioned in product descriptions. 
According to the Nanotechnology Consumer Products Inventory, silver nanoparticles are currently claimed to be used in more than 400 consumer products (Nanotechnologies, 20I4). Silver nanoparticles have antimicrobial activity and are used in food packaging material, food supplements, odour-preventing textiles, cosmetics, kitchen utensils, toys, electronics, wound dressings, and room sprays (Nanotechnologies, 20I4, Wijnhoven et al., 2009). Silver (nano)particles and released ions exert antimicrobial properties by binding to sulphur- and phosphorous-containing biomolecules such as proteins and DNA, thereby potentially also causing damage to mammalian cells (Sotiriou and Pratsinis, 20I I, Sotiriou and Pratsinis, 2010, Lok et al., 2007, Morones et al., 2005, Xiu et al., 20I2). Besides worker exposure, the extensive use of silver nanoparticles in products might also lead to consumer exposure by inhalation of the silver nanoparticles that are used in spray applications. Although silver nanoparticles are currently claimed to be used in many products, it is not clear to what extend they induce adverse effects after inhalation during production and use.

Silver nanoparticles have been studied in several in vivo inhalation studies showing diverse outcomes. Some studies showed no induction of adverse effects (Sung et al., $20 \mathrm{IIb}$, Ji et al., 2007), while other studies reported adverse effects varying from a minimal inflammatory response to the presence of inflammatory lesions in the lungs (Stebounova et al., 20ria, Song et al., 20r2, Sung et al., 2008, Sung et al., 2009). Regarding the tissue distribution of the silver nanoparticles, some studies report a dosedependent increase in the silver concentration in the lungs and in the liver (Ji et al., 2007, Song et al., 2012, Sung et al., 2009). Two of these studies report a rather high amount of silver detected in the brain and the olfactory bulb (Ji et al., 2007, Sung et al., 2009), causing concerns that silver nanoparticles might induce toxicity in the brain.

The before mentioned inhalation studies show that silver nanoparticles can induce pulmonary inflammation and can decrease lung function, depending on the exposure time and dosage (Sung et al., 20 I Ib, Ji et al., 2007, Stebounova et al., 20 I Ia, Song et al., 20I 2, Sung et al., 2008, Sung et al., 2009). However, all of these studies focused on a single particle size. The previous studies did not take particle size and surface area into account as explaining variable, whereas these affect the internal dose and the interaction probability with cells. For particles to induce pulmonary inflammation, they must deposit in the alveolar region. The deposition of (nano)particles depends mostly on their (agglomerate) size. Nanoparticles with a primary or agglomerate particle size between IO and I0O $\mathrm{nm}$ will deposit more efficiently in the alveolar region compared to particles with an agglomerate particle size between O.I and I $\mu \mathrm{m}$ (Asgharian et al., 2009, ICRP, I994, Carvalho et al., 20 I I, Cassee et al., 2002, Braakhuis et al., 20I4b). At a similar mass based exposure dose, particles of different sizes will have a different deposition pattern in the lungs, and the deposited dose in the alveoli ultimately determines the extent of the pulmonary toxic effects. The previous studies did not link the deposited dose in the alveoli to the observed effects (Ji et al., 2007, Song et al., 2012, Stebounova et al., 20 I ra, Sung et al., 2009, Sung et al., 20 I ib, Sung et al., 2008). 
Until now, the formulation in which silver nanoparticles induce toxicity remains unclear. The effects might be caused by the silver nanoparticles itself, the released silver ions, or a combination of both. Next to this, it remains unclear to what extend the geometric size of silver particles affect the induction of pulmonary inflammation. Since particle size is the most important particle characteristic that determines the deposited dose in the lungs and is of influence on the dissolution rate of silver nanoparticles, the aim of this study is to investigate the influence of particle size on pulmonary toxicity of silver nanoparticles. We hypothesize that small silver nanoparticles will induce more prominent pulmonary toxicity compared to larger silver particles because of the larger deposited dose in the alveoli and the higher dissolution rate. In the present study, we tested the effects of a similar mass exposure concentration of I $5 \mathrm{~nm}$ and 4 IO $\mathrm{nm}$ silver (nano)particles after short-term nose-only inhalation exposure. The total lung burden was measured and used together with the exposure measurements as an input for the multiple path particle dosimetry (MPPD) model to estimate the alveolar dose. Transmission electron microscopy (TEM) was used to localize silver particles in the lung tissue and tissue distribution was measured to determine any differences in the kinetics of the silver particles. To determine the toxicity induced by $\mathrm{I} 5 \mathrm{~nm}$ and $4 \mathrm{IO} \mathrm{nm}$ silver (nano)particles, body weight, total blood cell counts, inflammatory and cell damage markers in the bronchoalveolar lavage fluid (BALF) and histology of the lungs were analysed. All endpoints were determined at 24 hours and 7 days after the last exposure to investigate the possible recovery of adverse effects.

\section{METHODS}

\section{Test material and characterization}

The test atmosphere was produced by mixing silver nanoparticles with HEPA filtered and conditioned $\left(50 \% \mathrm{RH}, 2 \mathrm{I}^{\circ} \mathrm{C}\right)$ compressed air. Depending on the target particle size, one of two types of particle generators was used. The silver nanoparticles were produced by a Palas GFG ı ooo (Palas GmbH, Karlsruhe, Germany) spark generator fitted with silver tipped copper electrodes. To generate the $15 \mathrm{~nm}$ nanoparticles the output of the generator was immediately diluted in conditioned air. Oxygen was added to the dilution air to compensate for the argon flow from the Palas, a final concentration of $20 \%$ oxygen in the airflow was maintained. The particle number concentration was controlled by setting the Palas spark frequency. The final condition of the aerosol $\left(55 \% \mathrm{RH}, 2 \mathrm{I}^{\circ} \mathrm{C}\right)$ was set by adjusting the relative humidity of the mixing air (figure I). $200 \mathrm{~nm}$ silver particles were purchased by NanoComposix, inc. San Diego, USA. The $200 \mathrm{~nm}$ silver particles were polyvinylpyrrolidone (PVP)-coated and supplied in solution of $\mathrm{I} \mathrm{mg} / \mathrm{ml}$ in MilliQ water. The silver particle solution was forced through a small nozzle of a Schlick spray nozzle where it was broken up in small droplets (nebulized) by compressed air. The flow of the solution was controlled using a $20 \mathrm{ml}$ Terumo syringe and a syringe pump (TSE model 540200, Bad homburg, Germany). The nebulized flow was mixed with 
compressed air in a heated mixing tube. After mixing and drying, the aerosol passed through a tube oven $\left(700^{\circ} \mathrm{C}\right)$ to fuse the aggregates into a single particle. The heat of the oven removed the PVP-coating of the silver particles. After the oven, the aerosol diluted further with conditioned air and was allowed to cool. The mass concentration was controlled by adjusting the syringe pump motor speed and thus the flow of the solution. The final condition of the aerosol $\left(55 \% \mathrm{RH}, 2 \mathrm{I}^{\circ} \mathrm{C}\right)$ was set by adjusting the relative humidity of the final mixing air (figure 2).

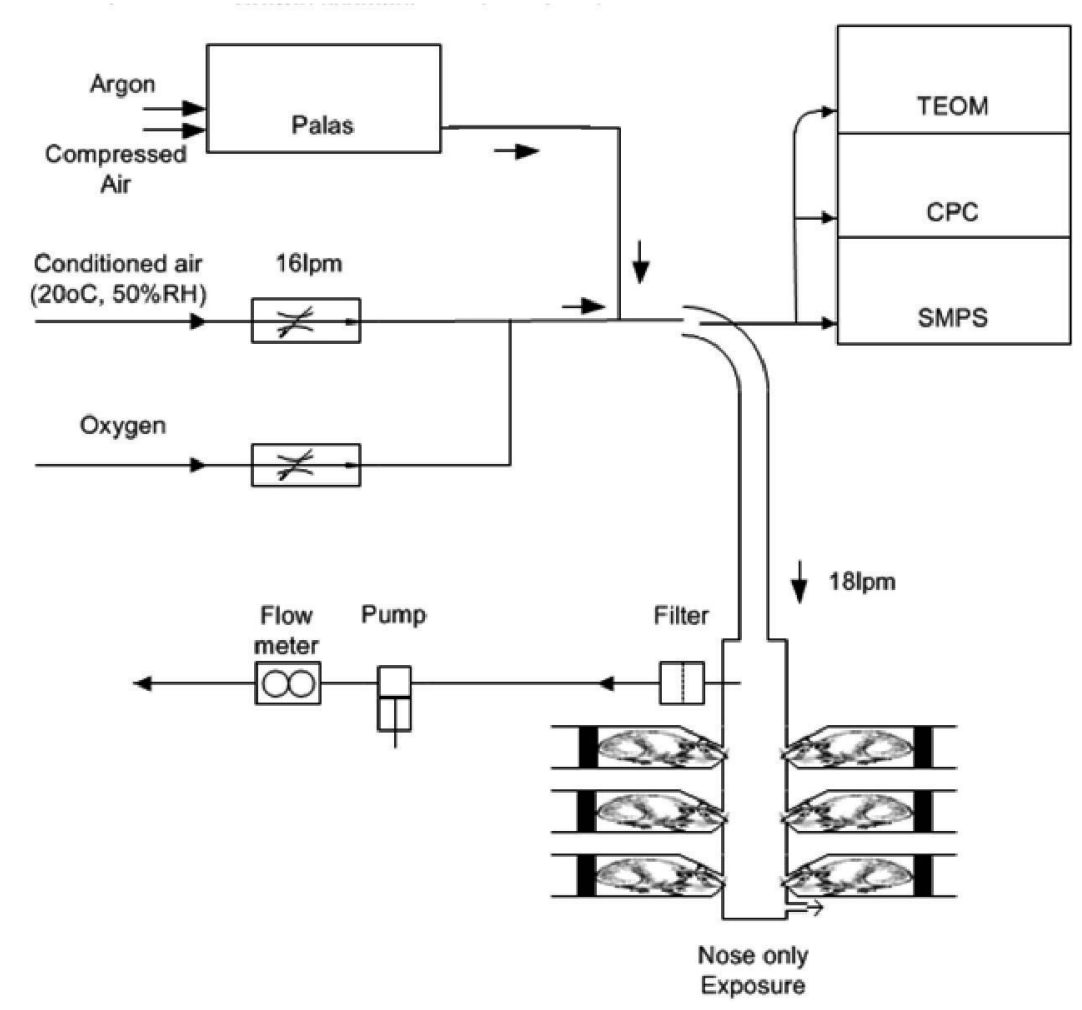

Figure 1. Palas spark generator setup for the nose-only exposure to $15 \mathrm{~nm}$ silver nanoparticles. The silver nanoparticles are generated by the Palas spark generator and subsequently mixed with conditioned air and oxygen. Particle size, number and mass are continuously monitored.

Several measurements of the test atmosphere were performed during the exposure. In the main flow upstream of the nose only unit particle mass concentration, number concentration, and particle size distribution were measured. The particle mass concentration was determined by time aggregated gravimetric with Teflon R2PJ047 filter (Pall corp., Ann Arbor MI, USA) and by tapered element oscillating microbalance (теом) seriesi 400 (Rupprecht \& Patashnick, New York, USA). The particle number 
concentration was measured over time by a condensation particle counter (CPC) 3022 (TSI inc., St Paul MN, USA). Particle size distribution was monitored over time by an OPS 3330 (TSI inc., St Paul MN, USA), a scanning mobility particle sizer (sMPS) 3080 with 3085 Nano Dma (TSI inc., St Paul mN, USA) and a mor Model No. i io (mSP corp, Minneapolis MN, USA). Temperature and relative humidity were determined by a Vaisala Mr7o (Vaisala Oyj, Helsinki, Finland). The particle size was confirmed by scanning electron microscopy (SEM) using a Nova Nanolab 600 Dualbeam (FEI, Eindhoven, the Netherlands) (figure I). In addition, the gravimetric mass concentration was determined. A Sartorius MC-5 microbalance (Sartorius, Goettingen, Germany) was used in controlled relative humidity $(40-45 \%)$ and temperature $\left(2 \mathrm{I}-23^{\circ} \mathrm{C}\right)$ conditions to do the mass measurements, the filters were weighed before and after each exposure. Laboratory and field blanks were used for quality assurance. The filter volume flow was measured with dry gas meters (Gallus 2000 Gi.6, Actaris Meterfabriek B.V., Dordrecht, the Netherlands).

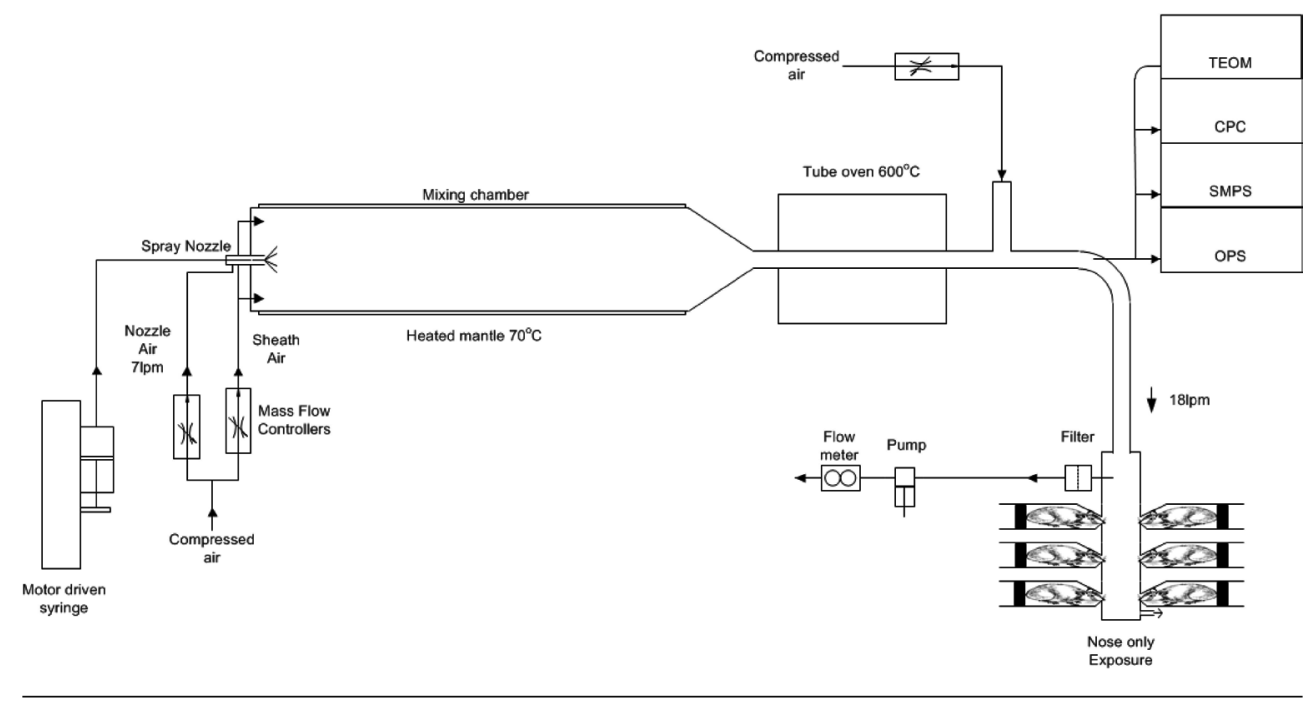

Figure 2. Spray nozzle setup for the nose-only exposure to $410 \mathrm{~nm}$ silver particles. The silver particles are in solution and sprayed into a heated mixing chamber. Subsequently, the particles are dried in an oven and mixed with compressed air. Particle size, number and mass are continuously monitored.

\section{Animals}

The Animal Ethical Committee of the National Institute for Public Health and the Environment (Bilthoven, the Netherlands) approved the experimental design of the animal study at the $6^{\text {th }}$ of July 20I2. Healthy male Fischer rats $\left(\mathrm{F}_{344} / \mathrm{DuCrl}\right)$ were supplied by Charles River (Sulzfeld, Germany). The animals were kept under specific pathogen free (SPF) conditions. After an acclimatization period of two 
weeks, the animals were ro weeks old at the start of the experiment. The rats were randomly allocated to the control and the test groups. During exposure, the animals were restrained in nose-only tubes ( $\mathrm{CH}$ technologies, Westwood NJ, USA) fixed to the inhalation system. When not exposed, animals were housed up to 3 animals in macrolon III cages with filtertops to prevent dust entering the cages (bedding material: Lignocel S8-I 5, Altromin Spezialfutter GmbH \& Co. KG). The animal room was airconditioned with a $\mathrm{I} 2$ hours light- $\mathrm{I} 2$ hours dark cycle, the temperature ranging from 20 to $24{ }^{\circ} \mathrm{C}$ with relative humidity ranging from $30-70 \%$. Except during exposure, certified feed CRM (sDs Diets, UK) and water were available ad libitum. Before start of the exposure period, all animals were acclimatized to the nose only tubes on 3 consecutive days for I hour per day. At 24 hours and 7 days after the last exposure, rats were weighed, anesthetized by a single intraperitoneal injection of ketamine (75 $\mathrm{mg} / \mathrm{kg}$ ) and xylazine ( $\mathrm{r} 0 \mathrm{mg} / \mathrm{kg}$ ) and subsequently exsanguinated via the abdominal aorta.

\section{Experimental design}

The rats were nose-only exposed for 6 hours per day, 4 consecutive days to fresh air, I $5 \mathrm{~nm}$ silver nanoparticles or $4 \mathrm{IO} \mathrm{nm}$ silver nanoparticles, I 2 rats per exposure group. Of each exposure group, 6 rats were sacrificed 24 hours after exposure and the other 6 rats were sacrificed 7 days after exposure. During nose-only inhalation, rats have a breathing pattern that results in a more realistic internal exposure than a single high dose in intratracheal instillation and oropharyngeal aspiration or possible additional oral exposure in whole-body exposure chambers.

\section{Quantification of silver in tissues by high resolution inductively coupled plasma mass spectrometry}

The presence of silver in the lungs, liver, spleen, kidneys, brain, testis and lung associated lymph nodes was determined by high resolution inductively coupled plasma mass spectrometry (HR-ICP-MS) (Krystek et al., 20 I 3, Krystek, 2O I 2). Samples of homogenized tissue (max. $0.5 \mathrm{~g}$ ) were completely dissolved on a block heater (Stuart SBH200D, supplied by Omnilabo, Breda, The Netherlands) for 24 hours at $120^{\circ} \mathrm{C}$ after the direct addition of aqua regia $(0.5 \mathrm{~mL} 60 \%$ nitric acid, ultrapure, and $\mathrm{I} .5 \mathrm{~mL} 30 \%$ hydrochloric acid, ultrapure) and afterwards diluted. The silver concentration in the digests was determined using high resolution inductively coupled plasma mass spectroscopy (HRICP-MS; Element XR, Thermo Fisher Scientific, Bremen, Germany). Both silver isotopes $\left({ }^{107} \mathrm{Ag}\right.$ and ${ }^{109} \mathrm{Ag}$ ) were measured in the low-resolution mode. External calibration curves of the interference-free silver isotopes were used for quantification. On-line addition and correction with an internal standard (rhodium with the measured isotope ${ }^{103} \mathrm{Rh}$ in the low-resolution mode) was applied too. The results of ${ }^{107} \mathrm{Ag}$ were reported and the results of ${ }^{109} \mathrm{Ag}$ were used for control. Sample pre-treatment and analysis were carried out in a cleanroom facility class Ioo ooo. 


\section{Estimated deposited dose in lungs using Multi Path Particle Dosimetry (MPPD) model}

To estimate the fractions of deposited dose in the head, tracheobronchial, and alveolar region of the rats, the multiple path particle dosimetry (MPPD) model was used (Asgharian et al., 2009). We used the default parameters of the model for rats, i.e., a forced respiratory capacity of $4 \mathrm{ml}$, head volume of $0.42 \mathrm{ml}$, nasal breathing, tidal volume of $2.1 \mathrm{ml}$, and a breathing frequency of $\mathrm{IO}_{0} / \mathrm{min}$. The inspiratory fraction was 0.5 , and no pause was entered. Calculations were done using the count median diameter (CMD), geometric SD, the mass concentration, and a density of $10.49 \mathrm{~g} / \mathrm{cm}^{3}$.

\section{Electron microscopy of the lung}

Of two animals of each group, the left lung was cut out with the bronchus attached and fixed by pressure ( $2 \mathrm{kPa}$ for I hour) with half-strength Karnovsky fixative (0.08 M Sodium-cacodylate buffer, $2.5 \%$ glutaraldehyde, $0.025 \mathrm{mM} \mathrm{CaCl}_{2}, 0.05 \mathrm{mM} \mathrm{MgCl}_{2}$ ) for electron microscopy. After two days, the fixative was replaced with $2 \%$ para-formaldehyde in sodium-cacodylate buffer for storage. Of each lung, three slices of about I - I.5 mm were cut out at the top, middle and bottom of the lung for further analysis. The tissue slices were post-fixed by osmium potassium ferro-cyanide solution (o.I M sodium-cacodylate buffer, I \% osmium tetra-oxide, I. $5 \%$ potassium ferro-cyanide). Next, the tissue slices were dehydrated in aceton series, cut in smaller pieces and subsequently embedded in epon. The epon was polymerized at $60^{\circ} \mathrm{C}$. The epon blocks were trimmed prior to sectioning. During sectioning, ultrathin sections of $70 \mathrm{~nm}$ were sectioned and put on $3 \mathrm{~mm}$ hexagonal copper grids coated with a formvarfilm. The sections were analysed by transmission electron microscopy (TEM) (Tecnai Io, IookV, FEI, Eindhoven, the Netherlands).

\section{Silver enhancement}

A silver enhancement procedure was used to increase the particle size of the silver nanoparticles in the lung tissue to make it possible to detect the silver nanoparticles with electron microscopy. The silver enhancement reagents were purchased at Aurion and performed on grid according to manufacturer's instructions (Wageningen, the Netherlands).

\section{Clinical examinations}

The animals were examined three times on exposure days (before, during and after exposure) and once daily during the periods before and after exposure. The clinical examination included examination of attitude, animal fur, activity level, food and water intake, and faeces and urine production. The body weight of the animals was determined upon arrival, at the start of the exposure period and at the day of sacrifice. 


\section{Haematology analysis}

Blood samples were collected in EDTA-containing tubes and in citrate vials for analysis of cell types and inflammatory markers in the blood. The level of Fibrinogen in the citrate plasma was measured using a rat fibrinogen ELISA kit (GenWay Biotech, Inc., San Diego, USA) according to manufacturer's instructions.

\section{Histopathology}

The lungs fixed with half-strength Karnovsky fixative were also used for histopathology. Of these lungs a thin slice was made over the length of the tissue, embedded in paraffin and stained by haematoxylin-eosin for histopathology analysis.

\section{Bronchoalveolar lavage fluid analysis}

The left lung of the rats was bound just below the bifurcation of the trachea and the right lung was cannulated via the trachea. Bronchoalvelar lavage was performed in situ by infusing the right lung three times with $27 \mathrm{ml} / \mathrm{kg}$ physiological saline. The retrieved bronchoalveolar lavage fluid (BALF) was kept on ice and centrifuged for Io minutes at $400 \mathrm{~g}$. The pellet was resuspended in physiological salt for analysis of the total cell number and cell differentials. Cytospin preparations were stained and evaluated microscopically for macrophages, polynuclear macrophages, polymorph nuclear neutrophils, lymphocytes, monocytes, eosinophils, and atypical cells. At least 400 cells were counted on each slide. The cell-free supernatant was collected to assess cell damage by measurement of total protein content and the release of lactate dehydrogenase (LDH) by Beckman Coulter autoanalyser Synchron LX2o (Beckman Coulter, Inc.) and to assess the induction of pro-inflammatory cytokines. The remainder of the supernatant was stored at $-80^{\circ} \mathrm{C}$.

\section{Measurement of pro-inflammatory cytokines}

The presence of pro-inflammatory cytokines IL-I beta, IL-6, TNF-alfa, IFN-gamma, IL-I 3 , GM-CSF, MCP-I, IL-I 2P7O, IL-I 8, MIP-Ia, MIP-2 and RANTES in the supernatant of the BALF was measured by a Bio-Plex Pro assay for rat cytokines according to manufacturer's instructions (Bio-Rad Laboratories, Inc.).

\section{Measurement of oxidative stress}

The induction of oxidative stress was determined by measuring the amount of reduced, oxidized and total glutathione in the lungs. The rinsed right lung was homogenized on ice in $4 \mathrm{ml}$ of Phosphate/EDTA buffer $(\mathrm{pH} 7.5)$. Subsequently, the homogenate was centrifuged at $600 \mathrm{~g}$ for 10 minutes. To remove the proteins from the samples, the supernatant was mixed I:I with metaphosphorid acid, incubated for Is minutes on 
ice, and centrifuged at $4000 \mathrm{rpm}$ for Io minutes. The amount of reduced, oxidized and total glutathione was measured by Beckmann Coulter Autoanalyser in the supernatant.

\section{Statistical analysis}

The raw data of the BALF was corrected for retrieved fluid. To compare the different exposure groups, the data was analysed by the Kruskal Wallis nonparametric test (Graphpad Prism). Statistical significance is indicated with $\mathrm{a}^{*}(p=<0.05)$. In all graphs, error bars represent the standard deviation of the mean.

\section{RESULTS}

\section{Nanoparticle characteristics}

The particle characteristics are summarized in table $\mathrm{I}$. The silver nanoparticles had a count median diameter of $\mathrm{I} 5 \mathrm{~nm}$ (figure 3 ) and the exposed dosage was $\mathrm{I} 79 \mu \mathrm{g} / \mathrm{m}^{3}$ air. For the larger silver particles, the $200 \mathrm{~nm}$ primary silver particles agglomerated to a count median diameter of $4 \mathrm{IO} \mathrm{nm}$ (figure 3) and the exposed dosage was $\mathrm{I} 67 \mu \mathrm{g} / \mathrm{m}^{3}$ air. While the mass dosage was similar for both particle groups, the particle number dosage was $3.8 \times \mathrm{IO}^{6}$ particles per $\mathrm{cm}^{3}$ for the $\mathrm{I} 5 \mathrm{~nm}$ nanoparticles and $2.0 \times 1 \mathrm{IO}^{4}$ particles per $\mathrm{cm}^{3}$ for the 4 IO $\mathrm{nm}$ particles.
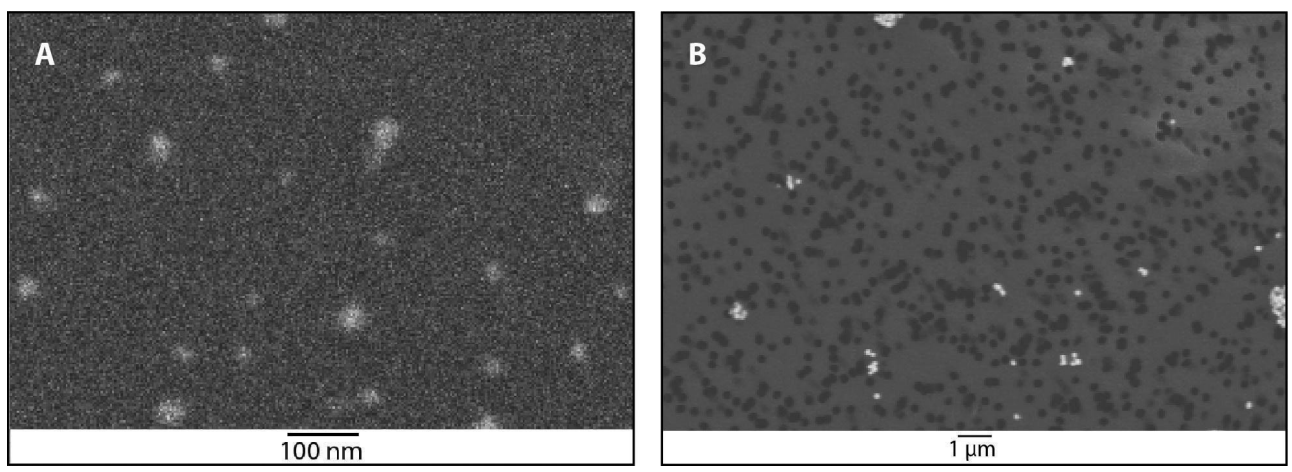

Figure 3. Scanning electron microscopy (sEM) pictures of the $15 \mathrm{~nm}$ silver nanoparticles (A) and the $410 \mathrm{~nm}$ silver particles (B) captured on polycarbonate filters with $0.22 \mu \mathrm{m}$ pores (Pictures are made in back-scatter mode). 
Table 1. Nanoparticle characteristics. Particle size is given as count median diameter (CMD) with the geometric standard deviation (GSD). Mass, particle number and surface area shown as mean with a $95 \%$ confidence interval.

\begin{tabular}{|c|c|c|c|}
\hline Agglomerate size $[\mathrm{nm}]$ & Mass $\left[\mathrm{\mu g} / \mathrm{m}^{3}\right]$ & $\begin{array}{c}\text { Particle number } \\
{\left[\# / \mathrm{cm}^{3}\right]}\end{array}$ & $\begin{array}{c}\text { Surface area } \\
{\left[\mathrm{nm}^{2} / \mathrm{cm}^{3}\right]}\end{array}$ \\
\hline $15.3(1.26)$ & $179(107-252)$ & $3.8 \times 10^{6}(2.6-5.0)$ & $6.72 \times 10^{9}(3.97-9.49)$ \\
\hline $410(1.43)$ & $167(147-187)$ & $2.0 \times 10^{4}(0.43-3.7)$ & $2.33 \times 10^{8}(0.28-2.61)$ \\
\hline
\end{tabular}

\section{Experimental design}

Since male rats showed more sensitivity towards induction of pulmonary inflammation compared to female rats after 90 days inhalation exposure (Song et al., 2012, Sung et al., 2008), we chose to expose male rats. The rats were nose-only exposed for 6 hours per day, 4 consecutive days to fresh air, I $5 \mathrm{~nm}$ silver nanoparticles or $4 \mathrm{IO} \mathrm{nm}$ silver particles, I 2 rats per exposure group. Of each exposure group, 6 rats were sacrificed 24 hours after exposure and the other 6 rats were sacrificed 7 days after exposure.

\section{Quantification of silver in tissues by HR-ICP-MS}

The presence of silver was measured with HR-ICP-MS in the lungs, liver, spleen, kidneys, brain, testis and lung-associated lymph nodes. Of these tissues, silver was detected in the lungs and liver (figure 4). At 24 hours after exposure, the lungs of the rats exposed to $\mathrm{I} 5 \mathrm{~nm}$ nanoparticles contained $3.4 \mu \mathrm{g}$ silver per gram lung, whereas the lungs of the rats exposed to $4 \mathrm{IO} \mathrm{nm}$ particles contained $6.0 \mu \mathrm{g}$ silver per gram lung. This equals a total lung deposit (TLD) of $5.5 \mu \mathrm{g}$ of the $\mathrm{I} 5 \mathrm{~nm}$ and $8.5 \mu \mathrm{g}$ of the $4 \mathrm{IO} \mathrm{nm}$ silver particles, respectively. Because the silver nanoparticles are spherical, we could use these measurements to estimate the number of silver nanoparticles deposited in the lungs. A total lung capacity (TLC) of I $3.7 \mathrm{ml}$ (Asgharian et al., 2009) was used that results in 2.0 $\mathrm{x} \mathrm{IO^{7 }}$ particles per $\mathrm{mm}^{3}$ of $\mathrm{I} 5 \mathrm{~nm}$ nanoparticles and $\mathrm{I} .6 \mathrm{x} \mathrm{IO}^{3}$ particles per $\mathrm{mm}^{3}$ of $4 \mathrm{IO}$ $\mathrm{nm}$ particles in the lungs. In the lung-associated lymph nodes, silver could be detected in one animal exposed to I $5 \mathrm{~nm}$ silver nanoparticles. In the other animals, the level of silver in the lymph nodes was below the detection limit. In addition, the level of silver in the other tested tissues (spleen, kidneys, testis, brain) was below the detection limit of 0.O I $\mu \mathrm{g} / \mathrm{g}$ tissue. At 7 days after exposure, the silver content in the lungs decreased to I.3 $\mu \mathrm{g} / \mathrm{g}$ tissue $(2 . \mathrm{I} \mu \mathrm{g}$ TLD) for the rats exposed to I $5 \mathrm{~nm}$ silver nanoparticles and $3.9 \mu \mathrm{g} / \mathrm{g}$ tissue $(5.9 \mu \mathrm{g}$ TLD) for the rats exposed to 4 I O nm silver particles. In the liver, silver was detected in the rats exposed to I $5 \mathrm{~nm}$ silver nanoparticles at a level of $0.06 \mu \mathrm{g} / \mathrm{g}$ tissue (0.53 $\mu \mathrm{g}$ total) at 24 hours after exposure and o.o I $\mu \mathrm{g} / \mathrm{g}$ tissue (0.095 $\mu \mathrm{g}$ total) at 7 days after exposure, indicating removal of silver. No silver was detected in the liver of rats exposed to 4IO nm silver particles at either time points. 
Ag in lung and liver $(\mu \mathrm{g} / \mathrm{g})$

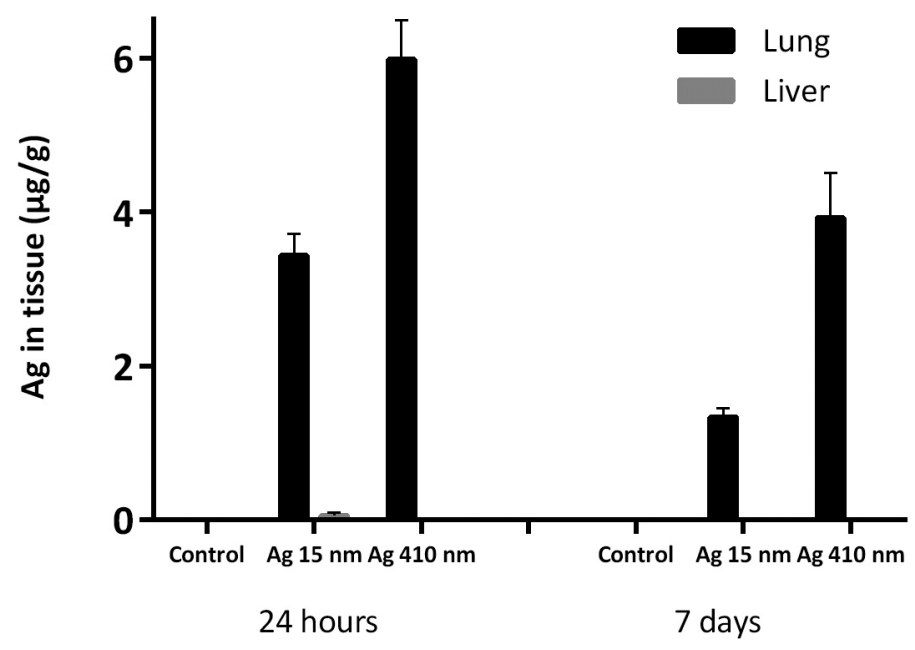

Figure 4. Amount of silver detected in the lungs and liver by HR-ICP-MS at 24 hours and 7 days after exposure. In the liver, silver could be detected in the animals exposed to $15 \mathrm{~nm}$ silver nanoparticles and not in the animals exposed to $410 \mathrm{~nm}$ silver particles. For both particle sizes, the amount of silver in the lungs at 7 days after exposure was significantly lower compared to 24 hours after exposure.

\section{Estimated deposited dose in lungs using Multiple Path Particle Dosimetry (MPPD) Model}

According to the MPPD model, the total deposited fraction in the lungs is similar for both particle sizes (Asgharian et al., 2009) at 0.69 for the $15 \mathrm{~nm}$ nanoparticles, and 0.67 for the 4IO $\mathrm{nm}$ particles. However, according to our HR-ICP-MS measurements, the TLD was $5.5 \mu \mathrm{g}$ for the $\mathrm{I} 5 \mathrm{~nm}$ nanoparticles and $8.5 \mu \mathrm{g}$ for the 4IO $\mathrm{nm}$ particles at 24 hours after exposure. The lower silver content in the lungs of the animals exposed to $15 \mathrm{~nm}$ particles might be explained by clearance of silver in the time between the end of the exposure and the sacrifice and after each day of exposure. The deposition in the alveoli differs between the two particle sizes: the deposited fraction in the alveoli is 0.27 for the $15 \mathrm{~nm}$ nanoparticles and 0.048 for the 4IO nm particles (Asgharian et al., 2009). Based on the HR-ICP-MS results, we estimated the deposited mass in the alveolar region was $2 . \mathrm{I} \mu \mathrm{g}$ for the $15 \mathrm{~nm}$ nanoparticles and $0.6 \mu \mathrm{g}$ for the $4 \mathrm{IO} \mathrm{nm}$ particles at 24 hours after exposure, which equals $7.9 \times 10^{6}$ particles per $\mathrm{mm}^{3}$ for the $\mathrm{I} 5 \mathrm{~nm}$ nanoparticles and I 8 particles per $\mathrm{mm}^{3}$ for the $4 \mathrm{IO} \mathrm{nm}$ particles. Based on mass, the deposited dose in the alveoli was 3.5 times higher in the animals exposed to $15 \mathrm{~nm}$ silver nanoparticles compared to the animals exposed to $4 \mathrm{IO} \mathrm{nm}$ silver particles at 24 hours after exposure. Based on particle number, the deposited dose in the alveoli was 66,000 times higher for the $15 \mathrm{~nm}$ compared to the 4IO $\mathrm{nm}$ particle group. 

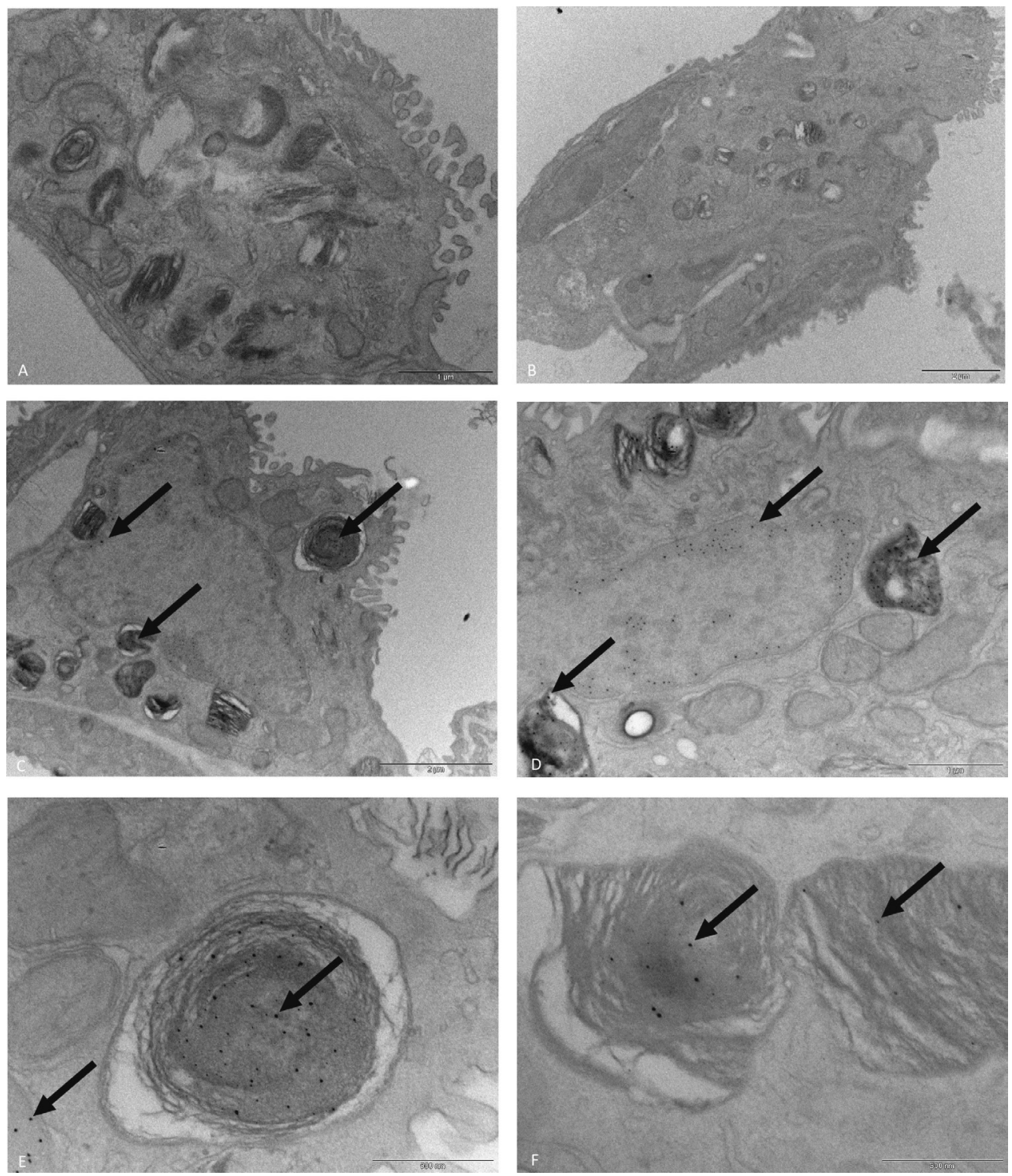

Figure 5. TEM pictures of lung tissue of rats exposed to clean air ( $A$ and $B$ ) or $15 \mathrm{~nm}$ silver nanoparticles ( $C, D, E$ and $F$ ), afterwards followed by silver enhancement. The pictures show the presence of silver nanoparticles in the nucleus, lamellar bodies and lysosomes of lung cells (indicated by the black arrows) at 24 hours after exposure.

\section{Electron microscopy of the lung}

As reported above, the estimated number of silver particles in the alveoli was $7.8 \times 10^{6}$ particles per $\mathrm{mm}^{3}$ for the I $5 \mathrm{~nm}$ nanoparticles and I 8 particles per $\mathrm{mm}^{3}$ for the $4 \mathrm{IO} \mathrm{nm}$ 
particles. The ultrathin TEM coupes are $70 \mathrm{~nm}$ thick; therefore, the expected number of particles per $\mathrm{mm}^{2}$ is 8 for the $4 \mathrm{I}$ O $\mathrm{nm}$ particles, which may be too few to be detected. In contrast, for the $15 \mathrm{~nm}$ nanoparticles, the expected number is much higher: $5.5 \times 10^{5}$ nanoparticles per $\mathrm{mm}^{2}$. Despite of this relatively high number neither I5 nor 4IO nm silver particles could be detected in TEM sections of the in epon embedded lung tissue.

Dissolution of silver nanoparticles is known to occur and will clearly result in a decrease in the size of the silver nanoparticles. Silver nanoparticles below $5 \mathrm{~nm}$ are difficult to detect in a biological sample by EM. Therefore, we employed the silver enhancement technique, a well-known approach to detect for instance I $\mathrm{nm}$ gold particles, normally not detectable in a biological sample by TEM. Silver enhancement also intensifies the EM signal of the silver nanoparticles by homogenous deposition of metallic silver on the particles surface, thereby increasing the particle size of the silver nanoparticles in the processed tissue. After silver enhancement on grids containing ultrathin lung sections, electron dense structures were detected by TEM in the lungs of the animals exposed to I $5 \mathrm{~nm}$ silver nanoparticles (figure 5 ), whereas they were almost absent in ultrathin lung sections of control animals. Two different grids containing ultrathin lung sections of control animals and three different grids containing ultrathin lung sections of animals exposed to $15 \mathrm{~nm}$ silver nanoparticles were examined for the presence of silver nanoparticles; at least 50 cell profiles per ultrathin section were examined. After silver enhancement, in the lung sections of the control animals silver dots were detected in $2 \%$ of the examined cells, whereas in the lung sections from the animals exposed to is $\mathrm{nm}$ silver nanoparticles silver dots were detected in $56 \%$ of the examined cells. The silver nanoparticles were both present in alveolar macrophages and lung epithelial cells (figure 5). The nanoparticles were located mostly in vesicle-like structures and in the nucleus (figure 5). However, in some cells the silver dots seemed randomly spread. The size of these deposits after silver enhancement was about $\mathrm{I} 5-20 \mathrm{~nm}$. This indicates that the original particle size of the silver nanoparticles in the lung tissue, without deposition of metallic silver, was smaller than $5 \mathrm{~nm}$ (P. van de Plas, Aurion, Wageningen, the Netherlands; personal communication).

Table 2. Animal weights given in grams. Data shown as mean with a $95 \%$ confidence interval.

\begin{tabular}{|c|c|c|c|c|}
\hline Group & Arrival & Start of exposure & $\begin{array}{c}\text { 24 hours after } \\
\text { exposure }\end{array}$ & $\begin{array}{c}\mathbf{7} \text { days after } \\
\text { exposure }\end{array}$ \\
\hline Control & $187(178-197)$ & $235(218-253)$ & $232(220-243)$ & $250(237-263)$ \\
\hline $15 \mathrm{~nm} \mathrm{Ag}$ & $188(179-197)$ & $237(228-245)$ & $231(222-240)$ & $248(243-253)$ \\
\hline $410 \mathrm{~nm} \mathrm{Ag}$ & $187(177-197)$ & $240(228-253)$ & $234(221-247)$ & $243(229-258)$ \\
\hline
\end{tabular}




\section{Clinical examinations}

Exposure to silver nanoparticles of $\mathrm{I} 5 \mathrm{~nm}$ and silver particles of $4 \mathrm{IO} \mathrm{nm}$ did not induce any premature mortality. There were no effects on animal attitude, fur, activity pattern, food and water consumption and faeces and urine production, and no effects on body weights and organ weights throughout the study (table 2).

\section{Haematology}

In the blood, the total amount of white blood cells and the amount of neutrophils and lymphocytes were all increased in the group exposed to the $15 \mathrm{~nm}$ silver nanoparticles compared to the other groups at 24 hours after exposure (figure 6). However, the differences were not statistically significant. At 7 days after exposure, there was no increase in any of the measured blood cells. There were no increases in inflammatory blood cells in the group exposed to 4IO nm silver particles at both time points. The level of Fibrinogen in the plasma did not differ between the controls and the exposed groups at both time points (data not shown).

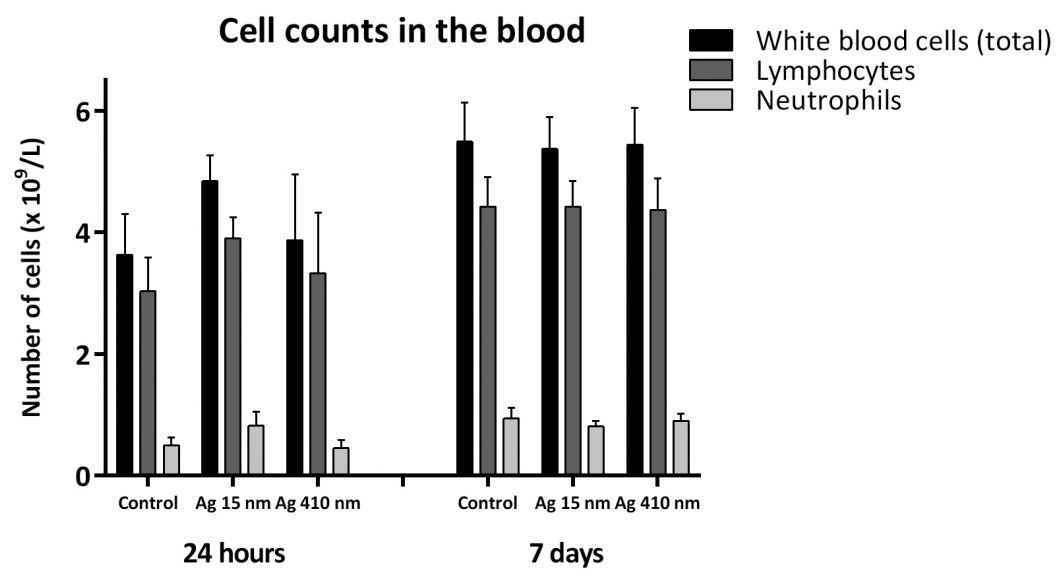

Figure 6. Number and type of cells in the blood after exposure to $15 \mathrm{~nm}$ and $410 \mathrm{~nm}$ silver particles. At 24 hours after exposure, the total amount of white blood cells was slightly increased in the group exposed to $15 \mathrm{~nm}$ silver nanoparticles compared to the controls as well as the number of neutrophils and lymphocytes. The differences were not statistically significant.

\section{Histopathology}

The histopathology analysis showed no differences between the groups. No lesions were observed in any of the lungs (results not shown). 


\section{Cell counts in the BALF}

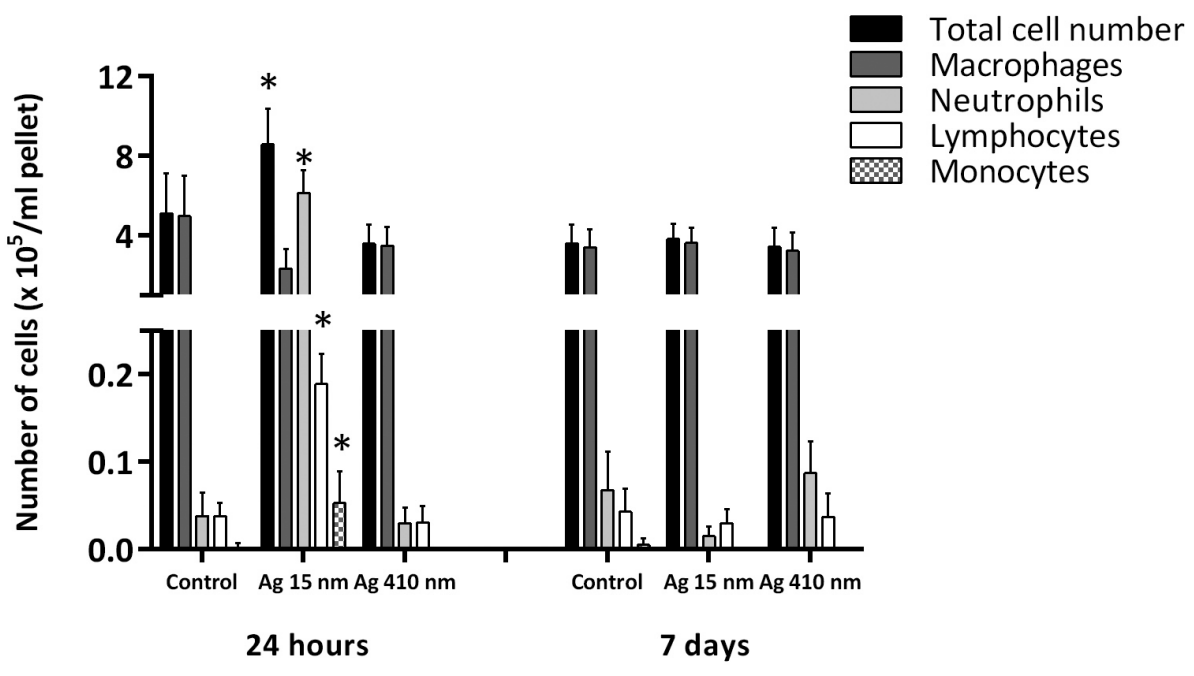

Figure 7. Cell counts in the BALF of rats. The group exposed to $15 \mathrm{~nm}$ silver particles had an increased number of total cells $(p=0.06)$, neutrophils $(p<0.01)$, lymphocytes $(p<0.05)$ and monocytes $(p<0.01)$ compared to the control group at 24 hours after exposure. Compared to the group exposed to $410 \mathrm{~nm}$ silver particles, the increased numbers of total cells ( $p<$ $0.05)$, neutrophils $(p<0.01)$, lymphocytes $(p<0.05)$ and monocytes $(p<0.001)$ were also significant. The increased cell numbers returned to normal at 7 days after exposure.

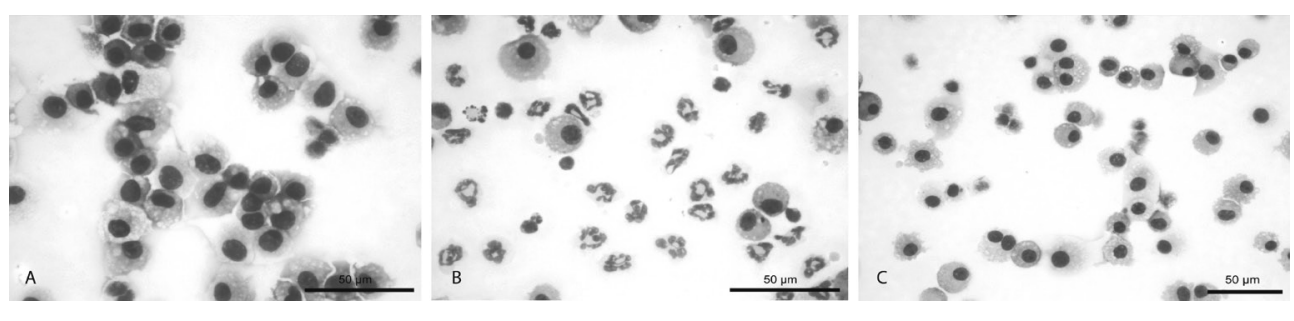

Figure 8. Light microscopic images of cells in the broncho-alveolar lavage fluid of the rats at 24 hours after exposure. A. Animal exposed to clean air. B. Animals exposed to $15 \mathrm{~nm}$ silver nanoparticles. C. Animals exposed to $410 \mathrm{~nm}$ silver particles. There is a significant influx of neutrophils in the lung fluid of the animals exposed to $15 \mathrm{~nm}$ silver nanoparticles. 


\section{Total cell counts and differential cell counts in the BALF}

The cell counts in the bronchoalveolar lavage fluid (BALF) are shown in figure 7. The total cell number was increased 24 hours after exposure to I $5 \mathrm{~nm}$ silver nanoparticles compared to control $(p=0.063)$ and significantly increased compared to the group exposed to 4IO $\mathrm{nm}$ silver particles $(p<0.05)$. The increased cell number is caused by a neutrophil influx (figure 8). 24 hours after exposure, there was an average of $3.95 \times 10^{5}$ neutrophils per $\mathrm{ml}$ cell pellet in the group exposed to $\mathrm{I} 5 \mathrm{~nm}$ silver nanoparticles compared to $2.3 \times \mathrm{IO}^{3}$ neutrophils per $\mathrm{ml}$ pellet in the controls $(p<0.0 \mathrm{I})$ and 2.I x $\mathrm{IO}^{3}$ neutrophils per $\mathrm{ml}$ pellet in the $4 \mathrm{IO} \mathrm{nm}$ silver particles exposed animals $(p<0 . \mathrm{OI})$. In addition, the number of lymphocytes in the BALF was increased after exposure to $\mathrm{I} 5 \mathrm{~nm}$ silver nanoparticles ( $p<$ 0.05 compared to control, $p<0.05$ compared to 4 IO $\mathrm{nm}$ silver). The number of monocytes in the BALF 24 hours after exposure was also increased: 3300 monocytes per $\mathrm{ml}$ pellet in the group exposed to $15 \mathrm{~nm}$ silver nanoparticles compared to 150 in the controls and o in the group exposed to $4 \mathrm{IO} \mathrm{nm}$ silver particles ( $\mathrm{p}<$ o.oI and $\mathrm{p}<0.00 \mathrm{I}$ ). Remarkably, the number of macrophages in the BALF was decreased 24 hours after exposure to $15 \mathrm{~nm}$ silver nanoparticles compared to control, although not statistically significant. There were no differences between the groups in number of multi-nuclei macrophages. There were no eosinophils detected in any of the exposed groups 24 hours after exposure.

The cell counts in the BALF at 7 days after exposure were completely different from those at 24 hours after exposure. At 7 days after exposure, most observed effects disappeared. There were no differences in the number of lymphocytes, macrophages and monocytes between the exposure groups. There was a slight decrease in the number of neutrophils in the BALF of rats exposed to I $5 \mathrm{~nm}$ silver nanoparticles compared to both control and 4IO nm silver particles, although not statistically significant.

For the groups exposed to $15 \mathrm{~nm}$ silver nanoparticles, the cell counts were significantly different between 24 hours and 7 days after exposure, for all cell types. Compared to 24 hours after exposure, the total cell number, neutrophils, lymphocytes and monocytes all significantly decreased and the number of macrophages, multi-nuclei macrophages significantly increased at 7 days after exposure.

\section{LDH and total protein in the BALF}

The supernatant of the BALF was used to determine lactate dehydrogenase (LDH) release and total protein as markers for cell damage (figure 9). At 24 hours after exposure, the groups exposed to I5 $\mathrm{nm}$ silver nanoparticles and 4IO $\mathrm{nm}$ silver particles both showed significantly increased levels of total protein in the BALF compared to the controls $(p<$ 0.01 and $\mathrm{p}<0.05$ respectively). The level of $\mathrm{LDH}$ was doubled at 24 hours after exposure to I $5 \mathrm{~nm}$ silver nanoparticles compared to both control $(p=0.05)$ and compared to $4 \mathrm{IO}$ $\mathrm{nm}$ silver particles exposed animals $(p<0.05)$. At 7 days after exposure, the levels of both total protein and LDH in the BALF of the exposed animals decreased to control levels. 


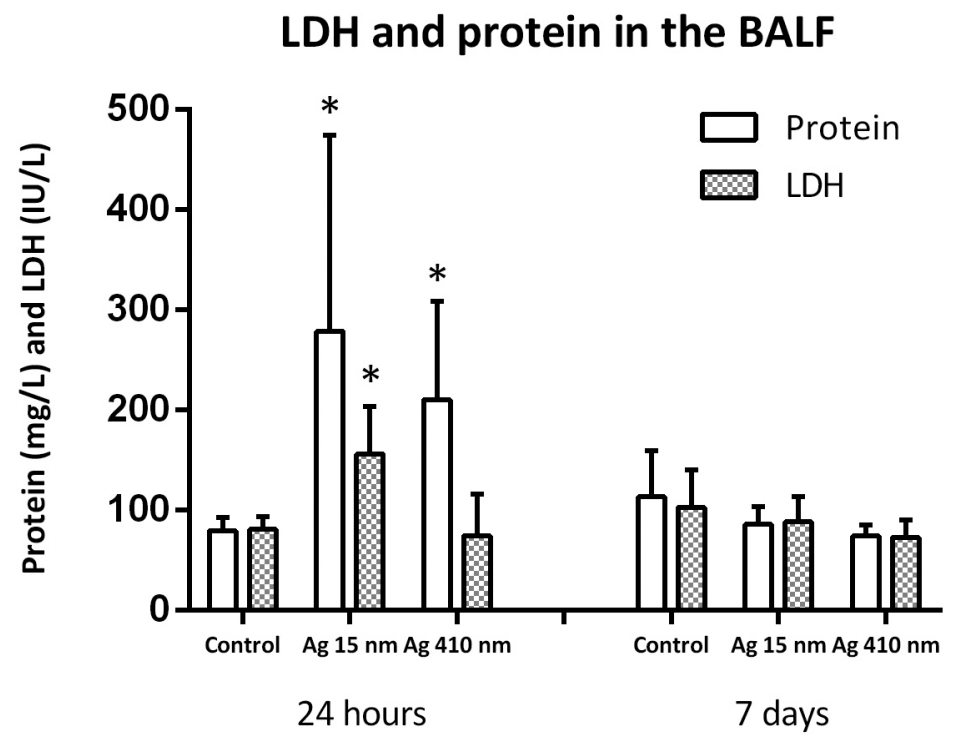

Figure 9. The level of total protein and LDH in the supernatant of the broncho-alveolar lavage fluid. At 24 hours after exposure, both the animals exposed to $15 \mathrm{~nm}$ silver particles and 410 $\mathrm{nm}$ silver particles showed a significant increase in the level of total protein compared to control ( $p<0.01$ and $p<0.05$, respectively). The total protein levels returned to normal at 7 days after exposure. The level of LDH was also significantly increased in the animals exposed to $15 \mathrm{~nm}$ silver nanoparticles at 24 hours after exposure compared to the control $(p<0.05)$ and to the animals exposed to $410 \mathrm{~nm}$ silver particles $(p<0.05)$.

\section{Pro-inflammatory cytokines in the BALF}

The supernatant of the BALF was used to determine the level of pro-inflammatory cytokines. I 2 different pro-inflammatory cytokines were selected: IL-I $\beta$, IL-6, TNF- $\alpha$, IFN- $\gamma$, IL-I 3 , GM-CSF, MCP-I, IL-I 2P7O, IL-I 8, MIP-Ia, MIP-2 and RANTES. Of these cytokines, only ILI $\beta$, MCP-I and MIP- 2 could be measured; all the other cytokines were and stayed below or around the detection level (figure IO). Both IL-I $\beta$ and MCP-I were significantly increased 24 hours after exposure to I $5 \mathrm{~nm}$ silver nanoparticles. IL-I $\beta$ was detected at $30.2 \mathrm{pg} / \mathrm{ml}$ for the I $5 \mathrm{~nm}$ nanoparticles exposed group compared to $5.5 \mathrm{pg} / \mathrm{ml}$ for the controls $(p<$ $0.05)$ and $4.9 \mathrm{pg} / \mathrm{ml}$ for the 4 IO $\mathrm{nm}$ particles exposed group $(p<0.05)$. In addition, the level of MCP-I was increased in the rats exposed to I $5 \mathrm{~nm}$ nanoparticles at 24 hours after exposure: $382.7 \mathrm{pg} / \mathrm{ml}$ compared to $6 \mathrm{I} .6 \mathrm{pg} / \mathrm{ml}$ of the controls $(p=0.063)$ and 42.8 in rats exposed to $4 \mathrm{IO} \mathrm{nm}$ silver particles $(p<0.0 \mathrm{I})$. The level of MIP-2 was significantly decreased to $26.5 \mathrm{pg} / \mathrm{ml}$ after 24 hours after exposure to I $5 \mathrm{~nm}$ silver nanoparticles compared to the level of $60.2 \mathrm{pg} / \mathrm{ml}$ in the controls $(p<0.0 \mathrm{I})$. The changes in IL-I $\beta$, MCP-I and MIP-2 levels at 24 hours after exposure to I $5 \mathrm{~nm}$ silver nanoparticles recovered 
at 7 days after exposure, when no significant differences were observed in any of the cytokine levels measured between the different exposure groups. There were no significant differences between the group exposed to 4IO nm silver particles and the controls at both 24 hours and 7 days after exposure.

\section{Pro-inflammatory cytokines in the BALF}

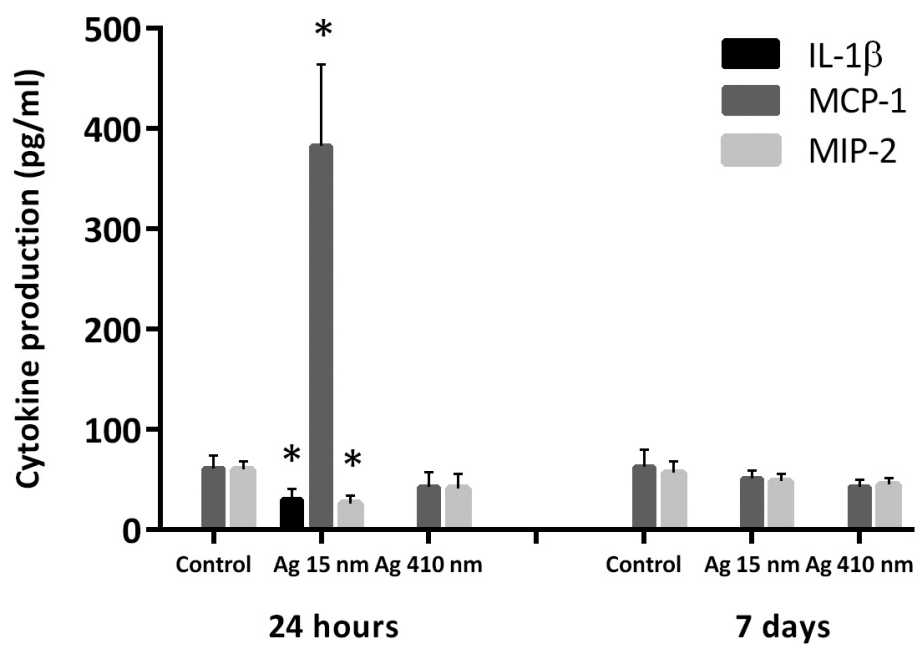

Figure 10. Cytokine level in the broncho-alveolar lavage fluid. The animals exposed to $15 \mathrm{~nm}$ silver nanoparticles had significantly increased levels of IL-1 $\beta(p<0.05)$, MCP-1 $(p=0.06)$ and MIP-2 $(p<0.01)$ in the BALF at 24 hours exposure compared to the controls. At 24 hours after exposure, the levels of IL-1 $\beta(p<0.05)$ and MCP-1 $(p<0.01)$ were also significantly increased compared to the animals exposed to $410 \mathrm{~nm}$ silver particles. At 7 days after exposure, the cytokine levels returned to normal.

\section{Oxidative stress in the lungs}

The levels of total glutathione, oxidized (GSSG) and reduced (GSH) glutathione were measured in the homogenate of the rinsed right lung of all the rats (figure II). The level of total glutathione was significantly increased at 24 hours after exposure to $15 \mathrm{~nm}$ silver nanoparticles compared to the controls $(p<0.0 \mathrm{I})$ and compared to the group exposed to 4IO $\mathrm{nm}$ silver particles $(p<0.05)$. The level of reduced glutathione was also significantly increased at 24 hours after exposure to $\mathrm{I} 5 \mathrm{~nm}$ silver nanoparticles compared to the controls $(p<0.0 \mathrm{I})$ and the 4IO nm exposed group $(p<0.05)$, whereas the level of oxidized glutathione was not. The ratio of GSH/GssG was not significantly different between any of the exposure groups. 7 days after exposure, the level of total glutathione and reduced glutathione in the group exposed to $15 \mathrm{~nm}$ silver nanoparticles returned to control levels. 


\section{Glutathione level in the lungs}

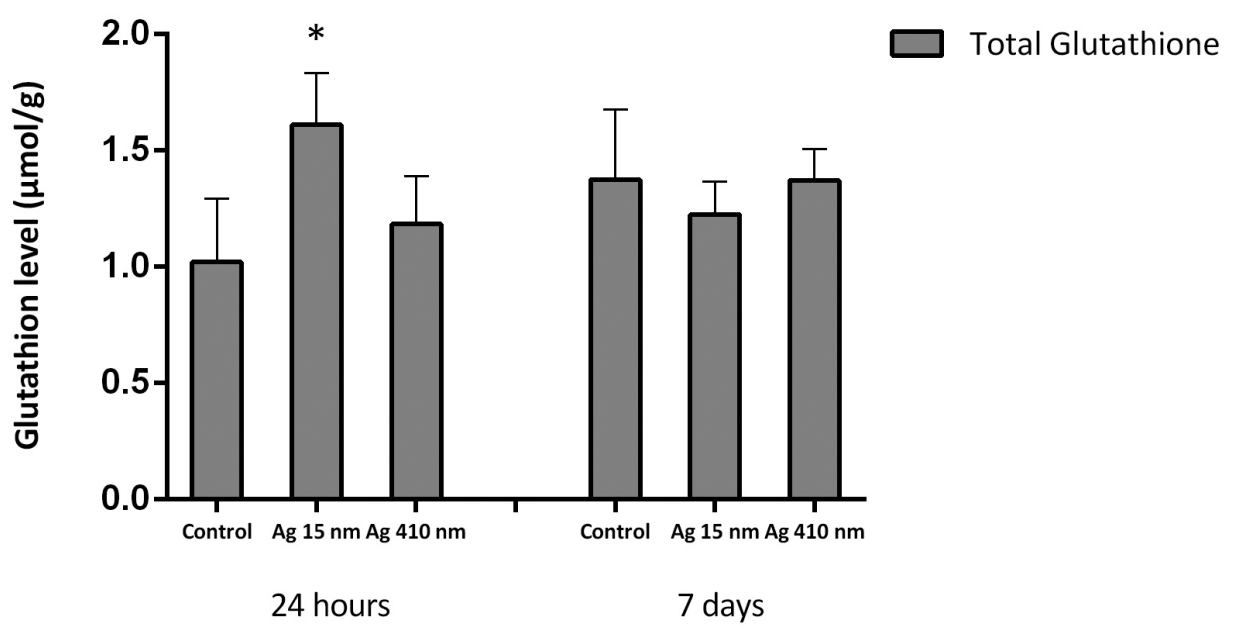

Figure 11. Glutathione production in the lungs of rats. At 24 hours after exposure, the total glutathione production in the $15 \mathrm{~nm}$ silver nanoparticles exposed group was significantly elevated compared to the controls $(p<0.01)$ and the $410 \mathrm{~nm}$ silver particles exposed group $(p<0.05)$.

\section{DISCUSSION}

In this short-term inhalation study, silver nanoparticles of two different sizes were tested to determine the differences in the lung deposition, tissue distribution and adverse effects. Exposure to $15 \mathrm{~nm}$ silver nanoparticles induced a moderate inflammatory response at 24 hours after the end of the exposure period. All the observed effects disappeared at 7 days after exposure. The most pronounced observed effect was the influx of neutrophils at 24 hours after exposure to $15 \mathrm{~nm}$ silver nanoparticles, which was about 175 times larger compared to the controls. In addition, we found release of pro-inflammatory cytokines, an increase in total glutathione and the presence of silver nanoparticles inside lung cells. In contrast, the 4IO nm particles induced none of these effects in the lung, clearly demonstrating a particle size dependent difference in the induction of pulmonary inflammation.

There are two main reasons to explain the noted differences in effects by the two silver particles. First, the differences in effects may be attributed to the differences in lung deposition of the smaller versus the larger silver particles. The total lung deposit as measured by HR-ICP-MS was $5.5 \mu \mathrm{g}$ for the $\mathrm{I} 5 \mathrm{~nm}$ silver nanoparticles and $8.5 \mu \mathrm{g}$ for the $4 \mathrm{IO} \mathrm{nm}$ silver particles at 24 hours after the end of the exposure period. According to the MPPD model, the fraction that reaches the alveoli is about 5.5 times higher for the 
I $5 \mathrm{~nm}$ nanoparticles compared to the $4 \mathrm{IO} \mathrm{nm}$ particles. Based on the HR-ICP-MS results we estimated the amount of I $5 \mathrm{~nm}$ silver nanoparticles present in the alveoli was $7.9 \mathrm{x}$ $\mathrm{IO}^{6}$ particles per $\mathrm{mm}^{3}$ (2.I $\mu \mathrm{g}$ mass or $5.8 \times \mathrm{IO}^{\mathrm{I2}} \mathrm{nm}^{2} / \mathrm{cm}^{3}$ surface area) and the number of $4 \mathrm{IO} \mathrm{nm}$ silver particles was I I 8 particles per $\mathrm{mm}^{3}\left(0.6 \mu \mathrm{g}\right.$ mass or $6.2 \mathrm{x} \mathrm{IO}^{\mathrm{IO}} \mathrm{nm}^{2} / \mathrm{cm}^{3}$ surface area). Therefore, the number of bioavailable $\mathrm{I} 5 \mathrm{~nm}$ nanoparticles in the alveoli is about 66,000 times larger compared to the $4 \mathrm{IO} \mathrm{nm}$ particles, resulting in a higher interaction probability with cells.

Comparing same mass deposits of microparticles and nanoparticles, the latter contains a much higher number of particles that need to be cleared. In addition, the smaller silver nanoparticles deposit deeper in the lungs where they are less efficiently cleared compared to the larger particles that deposit more in the conducting airways where they are more easily cleared (ICRP, I 994, Asgharian et al., 2009, Carvalho et al., 20 i I, Geiser and Kreyling, 2010, Oberdorster, I989, Oberdorster et al., I994, Geraets et al., 2012). Moreover, the phagocytosis of nanoparticles by macrophages is less efficient (Phalen et al., 20 Iо, Oberdorster and Pott, I987, Oberdorster et al., 2000) and slower compared to microparticles (Bakand et al., 20 I 2, Muhlfeld et al., 2008a, Geiser et al., 2008). Our results, however, show a clearance from the lungs of $62 \%$ of the $15 \mathrm{~nm}$ nanoparticles compared to $3 \mathrm{I} \%$ of the $4 \mathrm{IO} \mathrm{nm}$ particles at 7 days after exposure. The higher clearance rate of the smaller particles might be explained by translocation from the lungs of the smaller silver nanoparticles or the released ions to the blood or interstitium. This is further supported by the detection of silver in the liver of animals at 24 hours after exposure to I $5 \mathrm{~nm}$ silver nanoparticles, indicating systemic availability of silver particles or ions. This was not detected after exposure to the $4 \mathrm{IO} \mathrm{nm}$ silver particles.

Previous inhalation studies with nanosilver showed a dose-dependent increase in silver concentration in the liver (Ji et al., 2007, Song et al., 20 I 2, Sung et al., 2009) and even a low translocation to the brain and olfactory bulb (Ji et al., 2007, Sung et al., 2009). In one study, the amount of silver in the brain and olfactory bulb was $2.2 \mathrm{ng} / \mathrm{g}$ and $6.97 \mathrm{ng} / \mathrm{g}$, respectively, after exposure to the highest dose tested of $6 \mathrm{I} \mu \mathrm{g} / \mathrm{m}^{3}$ (Ji et al., 2007). In the other study, the amount of silver in the brain and olfactory bulb was I $8.63 \mathrm{ng} / \mathrm{g}$ and $30.48 \mathrm{ng} / \mathrm{g}$ at the highest dose tested of $5 \mathrm{I} 5 \mu \mathrm{g} / \mathrm{m}^{3}$ (Sung et al., 2009). In both studies, the tissue distribution of silver was detected by atomic absorption spectrophotometer. The reported level of silver is too low to detect with the HR-ICP-MS, which has a detection limit of o.o I $\mu \mathrm{g} / \mathrm{g}$, possibly explaining why we did not detect any silver in the brain or olfactory bulb. It should be noted that both detection methods do not distinguish between silver particles and silver ions.

The second reason for the observed differences in effects between the $\mathrm{I} 5 \mathrm{~nm}$ and $4 \mathrm{I} 0 \mathrm{~nm}$ silver particles is the difference in particle dissolution. It is known that smaller particles dissolve faster compared to larger particles because of the high surface to volume ratio (Ma et al., 2012). Several in vitro studies determined the dissolution rate of silver nanoparticles. In the study of Ma et al. (20 I I), the dissolution of silver nanoparticles 
ranged from $\mathrm{I} \%$ for the larger particles of $80 \mathrm{~nm}$ to $60 \%$ for the smallest particles of $5 \mathrm{~nm}$ at $\mathrm{pH} 8$ during three and two months incubation, respectively. However, there was no constant release of silver ions per unit of surface area, indicating that surface area alone did not explain the dissolution of silver nanoparticles (Ma et al., 2012). According to this and other studies (Ma et al., 20I2, Leo et al., 2013, Stebounova et al., 20I Ib, Kent and Vikesland, 20I2, Zook et al., 20I I), the dissolution of silver nanoparticles depends on their particle size, the $\mathrm{pH}$ of the solution, the ions present in the solution (e.g. sulphate, chloride and phosphate causing precipitations with silver ions or catalysing dissolution), aggregation state and the incubation time. Giving these in vitro results, the dissolution rate of the $15 \mathrm{~nm}$ silver nanoparticles will probably be higher compared to the 4IO $\mathrm{nm}$ silver particles resulting in an increased ion release. None of these studies report complete dissolution of silver nanoparticles, the effects observed after exposure to silver nanoparticles can be induced by the released ions, the silver nanoparticles itself or a combination of both.

In a study of Pratsinis et al (2013), the toxicity of silver nanoparticles in the presence of their released ions, and the released ions alone was tested in vitro. For small silver nanoparticles with a size below Io $\mathrm{nm}$, the released silver ions from the surface dominated the cytotoxicity whereas for larger silver nanoparticles the interactions with silver particles dominated the toxicity (Pratsinis et al., 2013). According to Beer et al. (20I2), at silver ion fractions $\leq 2.6 \%$ the silver nanoparticles contribute to the observed toxicity while at silver ion fractions $\geq 5.5 \%$ the silver nanoparticles do not add additional toxicity in vitro (Beer et al., 20I2). In a recent published study (Wang et al., $20 \mathrm{I} 4 \mathrm{~b}$ ), oropharyngeal aspiration in mice of $20 \mathrm{~nm}$ citrate-coated silver nanoparticles caused pulmonary inflammation indicated by neutrophil influx and the release of proinflammatory cytokines, whereas I Io nm citrate-coated silver particles caused a smaller neutrophil influx, only at the highest dose tested, and no release of pro-inflammatory cytokines. At 2 I days after exposure, the silver content in the lungs did not differ between $20 \mathrm{~nm}$ and I I $\mathrm{nm}$ silver particles. In addition, for both particle sizes, there was no silver detected in any of the other selected organs and tissues. Solubility test showed that about $4 \%$ of the $20 \mathrm{~nm}$ nanoparticles and $2 \%$ of the I I $\mathrm{nm}$ particles dissolved in 24 hours. Therefore, the authors concluded the larger pulmonary inflammation caused by the $20 \mathrm{~nm}$ silver nanoparticles is due to the higher bioavailability of silver ions. However, the effects of $20 \mathrm{~nm}$ silver nanoparticles were stronger compared to the effects of silver nitrate indicating the particles itself contributed to the observed effects. In contrast to these results, the I I $\mathrm{nm}$ silver particles caused mild sub-chronic effects in the lungs at 2I days after exposure, which might be caused by a slower and more persistent silver ions release (Wang et al., 20I4b). Similar to these studies, it is likely that the release of silver ions contributed to the observed effects after exposure to I 5 $\mathrm{nm}$ silver nanoparticles in the present study. To further elucidate the mechanism of the silver nanoparticle toxicity, the dissolution behaviour of silver nanoparticles inside cells should be investigated in detail. 
In our study, we could detect by TEM silver nanoparticles in the lung tissue of rats at 24 hours after exposure to $\mathrm{I} 5 \mathrm{~nm}$ silver nanoparticles. At 24 hours after exposure to $4 \mathrm{IO}$ $\mathrm{nm}$ silver particles, we could not detect silver particles in the lungs because the number of particles was too low. The detected silver nanoparticles had a particle size of $15-20$ $\mathrm{nm}$ after silver enhancement, indicating their size in the lung tissue was smaller than 5 $\mathrm{nm}$ before enhancement. In combination with the literature on the dissolution of silver nanoparticles, these EM results are an indication that the silver nanoparticles probably partially dissolved after deposition, resulting in a decrease in particle size and the release of silver ions. The dissolution might occur extra-cellular in the lung lining fluid and intracellular in vesicles in epithelial cells or macrophages. Besides the release of silver ions, we believe the silver nanoparticles itself may also have contributed to the observed effects because the TEM images show the presence of silver nanoparticles inside lung cells. The I $5 \mathrm{~nm}$ silver nanoparticles in our study dissolved over time resulting on the one hand in the release of toxic silver ions and on the other hand in a decrease in particle size to $<5 \mathrm{~nm}$ which gives the particles the opportunity to pass the cellular membranes and the nuclear pores. Previous studies report that particles smaller than $5 \mathrm{~nm}$ possibly can pass the nuclear pores (Singh et al., 20Io), while particles of $20 \mathrm{~nm}$ do not reach the nucleus and could be detected in vesicles and lamellar bodies in vitro (Herzog et al., 2013). Once inside the cells and eventually inside the nucleus, silver nanoparticles are a continued source of ion release, leading to more damage compared to silver ions released from silver nitrate (Lubick, 2008). The intracellular ion release has been reported previously as the Trojan-horse type mechanism (Park et al., 20 Io, Limbach et al., 2007); nanoparticles can more easily enter the cells compared to the ions itself. The higher dissolution rate, in combination with the higher alveolar deposition of the $15 \mathrm{~nm}$ nanoparticles, might explain why the I $5 \mathrm{~nm}$ nanoparticles caused toxicity and the $4 \mathrm{IO} \mathrm{nm}$ particles did not. Based on the results of the present study, it is not possible to quantify how much of the observed effects can be attributed to the difference in alveolar deposition and how much of the effects can be attributed to cellular uptake and ion release.

Findings of particle size related pulmonary inflammation are not limited to silver nanoparticles. Other inhalation studies with titanium dioxide, carbonaceous, nickel oxide, zinc oxide, ferric oxide, aluminium oxyhydroxide, quartz and cerium oxide particles also focused on the influence of particle size on the induction of pulmonary inflammation (Oberdorster et al., 2000, Stoeger et al., 2006, Horie et al., 20 I 2, Duffin et al., 2007, Kobayashi et al., 2009, Ho et al., 20 I I, Zhu et al., 2008, Pauluhn, 2009a, Roursgaard et al., 2010, Gosens et al., 2013). Indeed, several studies report that smaller nanoparticles caused a greater inflammatory response compared to larger particles when the same mass dosage was administered (Oberdorster et al., 2000, Stoeger et al., 2006, Horie et al., 20I2, Duffin et al., 2007, Kobayashi et al., 2009, Ho et al., 20 I I). However, although particle size will affect the site and amount of deposited material, it seems that the local dose alone cannot explain the differences in toxicity. Solubility will at least be one of the other variables in an equation that would allow predicting adverse effects of nanoparticles after inhalation. 


\section{CONCLUSION}

The results of the present short-term inhalation study of uncoated silver particles indicate that particle size is an important characteristic that determines the induction of pulmonary inflammation. Exposure to I $5 \mathrm{~nm}$ silver nanoparticles induced moderate pulmonary inflammation at 24 hours after exposure, whereas 4IO $\mathrm{nm}$ silver particles did not. The lung deposition of the 4IO nm silver particles was mainly in the upper airways; the number of particles deposited in the alveoli was calculated to be 66,000 times higher for the $15 \mathrm{~nm}$ silver nanoparticles. TEM analysis showed the presence of silver nanoparticles in lung cells that were decreased in particle size, demonstrating the in vivo dissolution of silver nanoparticles. Altogether, these findings strongly suggest that size-related silver nanoparticle induced pulmonary inflammation is a consequence of both size related lung deposition and dissolution rate.

\section{ACKNOWLEDGEMENTS}

This work was supported by the project 'Integrated Risk Assessment of Nanomaterials' from the National Institute for Public Health and the Environment and by the NanoNextNL program 'Risk Analysis and Technology Assessment: Human Health Risks'. We would like to thank Liset J.J. de la Foneteyne for the BALF and haematology cells counts, Piet K. Beekhof for the analysis of the total protein, LDH and glutathione, and Henny W. Verharen, Hans J.C. Strootman, Ron F. Vlug, Christine M.R. Soputan, Jan Bos, Jolanda Rigters for their excellent technical assistance. In addition, we thank Karin E. M. Vocking, Elly G. van Donselaar for their assistance with the TEM imaging, Jose van den Dungen for assistance with sample pretreatments and D. A. Matthijs de Winter for the assistance with the sEM imaging. 

Hedwig M. Braakhuis ${ }^{1,2^{*}}$, Flemming R. Cassee ${ }^{2,3}$, Paul H.B. Fokkens ${ }^{2}$, Liset J.J. de la Fonteyne ${ }^{2}$, Agnes G. Oomen ${ }^{2}$, Petra Krystek ${ }^{4}$, Wim H. de Jong ${ }^{2}$, Henk van Loveren ${ }^{\mathrm{I}, 2}$, Margriet V.D.Z. Park $^{2}$

Nanotoxicology 20 I 5 February 23: I-I I

${ }^{I}$ Department of Toxicogenomics, Maastricht University, Po Box 6I6, 6200 MD Maastricht, the Netherlands

${ }^{2}$ National Institute for Public Health and the Environment (RIVM), PO Box I, 3720 BA

Bilthoven, the Netherlands

${ }^{3}$ Institute of Risk Assessment Sciences, Utrecht University, po Box 80.163, 3508 TD Utrecht, the Netherlands

${ }^{4}$ Philips Innovation Services, 5656 AE Eindhoven, the Netherlands 


\section{Identification of the appropriate dose metric for pulmonary inflammation of silver nanoparticles in an inhalation toxicity study




\section{ABSTRACT}

A number of studies have shown that induction of pulmonary toxicity by nanoparticles of the same chemical composition depends on particle size, which is likely in part due to differences in lung deposition. Particle size mostly determines whether nanoparticles reach the alveoli, and where they might induce toxicity. For the risk assessment of nanomaterials, there is need for a suitable dose metric that accounts for differences in effects between different sized nanoparticles of the same chemical composition. The aim of the present study is to determine the most suitable dose metric to describe the effects of silver nanoparticles after short-term inhalation. Rats were exposed to different concentrations (ranging from $4 \mathrm{I}$ to I IOS $\mu$ g silver $/ \mathrm{m}^{3}$ air) of I $8 \mathrm{~nm}, 34 \mathrm{~nm}, 60 \mathrm{~nm}$ and $\mathrm{I} 60 \mathrm{~nm}$ silver particles for four consecutive days and sacrificed at 24 hours and 7 days after exposure. We observed an exposure concentration dependent increase in pulmonary toxicity parameters like cell counts and pro-inflammatory cytokines in the bronchoalveolar lavage fluid. All results were analysed using the measured exposure concentrations in air, the measured internal dose in the lung and the estimated alveolar dose. In addition, we analysed the results based on mass, particle number and particle surface area. Our study indicates that using the particle surface area as a dose metric in the alveoli, the dose-response effects of the different silver particle sizes overlap for most pulmonary toxicity parameters. We conclude that the alveolar dose expressed as particle surface area is the most suitable dose metric to describe the toxicity of silver nanoparticles after inhalation.

\section{INTRODUCTION}

Nanomaterials are composed of primary and agglomerated particles that can vary in size, shape, charge, crystallinity, chemical composition and other characteristics, and this variety will increase even further in the future (SCENIHR, 20Io). Of these characteristics, size has been put forward as an essential parameter to predict the pulmonary inflammation caused by nanomaterials (Oberdorster et al., 2000, Stoeger et al., 2006, Horie et al., 2012, Kobayashi et al., 2009, Duffin et al., 2007), although many other factors also modulate the outcome of toxicity studies (Braakhuis et al., 20I4b, Bakand et al., 2012, Cho et al., 2012b, Landsiedel et al., 2010, Ma-Hock et al., 2013, Schinwald et al., 20 I 2b, van Ravenzwaay et al., 2009, Warheit et al., 2007b, Warheit et al., 2007c). In inhalation studies using similar mass exposure concentrations of microparticles ( $>\mathrm{I} \mu \mathrm{m}$ ) and nanoparticles, differences in effects may be the result of differences in total surface area and total particle number, but also in lung deposition and clearance (Asgharian et al., 2009). In a recent study, silver nanoparticles of two different sizes showed a clear size-dependent effect after short-term in vivo inhalation; I $5 \mathrm{~nm}$ particles induced moderate pulmonary toxicity while 4IO nm particles did not (Braakhuis et al., 20I4a). The observed effects were likely caused by the difference in the internal alveolar dose between the $15 \mathrm{~nm}$ and 4IO $\mathrm{nm}$ silver particles and by the release 
of silver ions from the (nano)particle surface. Several other in vivo studies also showed that smaller nanoparticles caused more pronounced adverse effects after inhalation compared to larger particles of the same chemical composition when applying the same mass concentrations (Oberdorster et al., 2000, Stoeger et al., 2006, Horie et al., 20 I2, Duffin et al., 2007, Kobayashi et al., 2009). For nanoparticles studied in those experiments, the deposited particle surface area seems to be a better predictor for the induction of pulmonary inflammation than mass exposure concentrations. In other in vivo studies no correlations between decreasing primary particle size and increasing pulmonary inflammation were obtained (Zhu et al., 2008, Pauluhn, 2009a, Roursgaard et al., 20Io, Gosens et al., 20I4, Warheit et al., 2006). In two of these studies, the agglomerate particle size was similar for particles of different primary size, resulting in similar deposition patterns in the lungs (Gosens et al., 2014, Pauluhn, 2009a) and possibly explaining the absence of a primary particle size related effect.

For risk assessment purposes, for example to derive exposure limits, there is need for a suitable dose metric of nanomaterials that accounts for differences in effects between different sized nanoparticles of the same chemical composition. In the present study, we chose to focus on silver nanoparticles as these are currently the most common mentioned in product descriptions because of their antimicrobial activity (Nanotechnologies, 20I4, Wijnhoven et al., 2009). The aim of this study is to determine the most suitable dose metric to describe the effect of silver nanoparticles after inhalation irrespective of the size of the primary particle. From previous studies, it is known that silver nanoparticles can release silver ions from their surface (Ma et al., 20 2, Leo et al., 20 I3, Stebounova et al., 20IIb, Kent and Vikesland, 20I2, Zook et al., 20II) that contribute to the observed toxicity after inhalation (Pratsinis et al., 20I3, Beer et al., 20 I2, Wang et al., $2014 \mathrm{~b})$. Because of their high surface to volume ratio and the release of silver ions from the surface, we hypothesize that deposited particle surface area might be a suitable dose metric to predict the pulmonary toxicity of silver nanoparticles after inhalation.

To address this hypothesis, rats were exposed by inhalation to four different concentrations of I $8 \mathrm{~nm}, 34 \mathrm{~nm}, 60 \mathrm{~nm}$, and I60 nm silver (nano)particles for four consecutive days to obtain dose-response data. We measured the total lung burden and estimated the alveolar dose using a multiple path particle dosimetry (MPPD) model (Asgharian et al., 2009). We subsequently correlated various dose metrics with a suit of toxicity markers determined at 24 hours and 7 days after the last exposure.

\section{METHODS}

\section{Test material generation}

The test atmosphere was produced by mixing silver nanoparticles with HEPA filtered and conditioned $\left(50 \% \mathrm{RH}, 2 \mathrm{I}^{\circ} \mathrm{C}\right)$ compressed air. The silver nanoparticles were produced 
by a Palas GFG Iooo (Palas GmbH, Karlsruhe, Germany) spark generator fitted with silver tipped electrodes. To generate the smallest aerosol size (I 5-20 nm CMD), the output of the generator was immediately diluted in conditioned air. To generate the two intermediate aerosol sizes of $30-35 \mathrm{~nm}$ and $60-65 \mathrm{~nm}$, a longer residence time of spark generated particles in mixing chambers was used to lead the aerosol into the exposure chamber. This increased residence time resulted in aggregation, which is a function of time. In order to obtain spherical particles, the aerosol was heated by passing it via tubing through an oven at $700{ }^{\circ} \mathrm{C}$, which resulted in fusing of aggregates. Oxygen was added to the dilution and cooled air to a final concentration of $20 \%$. The particle number concentration was controlled by the spark generator frequency. The final condition of the aerosol $\left(55 \% \mathrm{RH}, 2 \mathrm{I}^{\circ} \mathrm{C}\right)$ was set by adjusting the relative humidity of the mixing air. It is not possible to use this spark generation method to produce silver nanoparticles larger than $60-65 \mathrm{~nm}$. Therefore, we used another generation method to generate larger silver nanoparticles.

$80 \mathrm{~nm}$ silver particles were purchased from NanoComposix, inc. (San Diego, USA). The $80 \mathrm{~nm}$ silver particles were polyvinylpyrrolidone (PVP)-coated and supplied in solution of I $\mathrm{mg} / \mathrm{ml}$ in MilliQ water. The silver particle suspension was passed through a small nozzle of a Schlick spray nozzle (Düsen-Schlick GmbH, Untersiemau / Coburg, Germany) to obtain an aerosol. The nebulized particles were mixed with compressed air in a heated mixing tube. After mixing and passing the aerosol through a diffusion dryer to get rid of the water, the aerosol was passed through an oven as described above. At temperatures above $500{ }^{\circ} \mathrm{C}$, pVP completely decomposes (Peniche et al., I993, Du et al., 2006). The high temperature of $700{ }^{\circ} \mathrm{C}$ removed the PVP-coating of the silver particles. After the oven, the aerosol was diluted with conditioned air and cooled down. The final condition of the aerosol $\left(55 \% \mathrm{RH}, 2 \mathrm{I}^{\circ} \mathrm{C}\right)$ was set by adjusting the relative humidity.

\section{Test atmosphere characterization}

The particle mass concentration was determined by gravimetric analysis of particles collected on Teflon R2PJo47 filter (Pall corp., Ann Arbor MI, USA). Mass concentrations were also monitored using a tapered element oscillating microbalance (TEOM) series 400 (Rupprecht \& Patashnick, New York, USA). The particle number concentration was measured by a condensation particle counter CPC 3022 (TSI inc., St Paul MN, USA). Particle size distribution was monitored by an optical particle sizer (OPS) 3330 (TSI inc., St Paul MN, USA), a scanning mobility particle sizer (SMPS) 3080 with 3085 Nano differential mobility analyser (DMA) (TSI inc., St Paul MN, USA) and a micro-orifice impactor (MOI) Model No. I Io (MSP corp, Minneapolis MN, USA). Temperature and relative humidity were determined continuously during the exposures using a Vaisala Mı70 (Vaisala Oyj, Helsinki, Finland). The particle size and spherical appearance was confirmed by scanning electron microscopy (SEM) using a Nova Nanolab 60o Dualbeam (FEI, Eindhoven, the Netherlands), about 300 particles per particle size were analysed using SEM. The measurements of particle mass, number, and particle size (count median 
diameter) were used to estimate the particle surface area in the external exposure air. This is assuming that the particles were spherical, which was confirmed by the SEM images of the particles (figure $S_{\mathrm{I}}$ ). For the internal doses, we measured the mass of the silver present in the lungs with high resolution inductively coupled plasma mass spectrometry (HR-ICP-MS). In combination with the particle size measured during exposure, we could estimate the particle surface area. For the alveolar dose, we used the MPPD model to estimate the mass fraction that would reach the alveoli and used the HR-ICP-MS results to calculate the mass that would reach the alveoli. Again, by using the particle size in combination with the mass we could estimate the particle surface area.

\section{ANIMALS}

Male Fischer rats ( $\left.\mathrm{F}_{344} / \mathrm{DuCrl}\right)$, specific pathogen free (SPF) were supplied by Charles River (Sulzfeld, Germany). After an acclimatization period of two weeks, the animals were Io weeks old at the start of the experiment. All animals were free from clinical signs of disease. The rats were randomly allocated to the control and the test groups. During exposure, the animals were restrained in nose-only tubes ( $\mathrm{CH}$ technologies, Westwood NJ, USA) fixed to the inhalation system. When not exposed, animals were housed up to 3 animals in macrolon III cages with filtertops to prevent dust entering the cages (bedding material: Lignocel S8-I 5, Altromin Spezialfutter GmbH \& Co. KG). The animals' room was air-conditioned with a I 2 hours light- I 2 hours dark cycle, the temperature ranging from 20 to $24^{\circ} \mathrm{C}$ with relative humidity ranging from $30-70 \%$. Except during exposure, certified feed CRM (sDs Diets, Essex, UK) and water were available ad libitum. Before start of the exposure period, all animals were acclimatized to the nose only tubes on 3 consecutive days for I hour per day. At 24 hours and 7 days after the last exposure, rats were weighed, anesthetized by a single intraperitoneal injection of ketamine ( 75 $\mathrm{mg} / \mathrm{kg}$ ) and xylazine ( $10 \mathrm{mg} / \mathrm{kg}$ ) and subsequently exsanguinated via the abdominal

aorta. Husbandry conditions were maintained according to all applicable provisions of the national laws, Experiments on Animals Decree and Experiments on Animals Act.

\section{Experimental design}

The experiments were conducted at the National Institute for Public Health and the Environment (RIVM, Bilthoven, The Netherlands) according to a protocol approved by the local Ethics Committee for Animal Experiments and according to European and national laws regulating the use of laboratory animals. The experimental design is shown in figure I. To obtain dose-response data, rats were exposed to different concentrations of the silver (nano)particles by exposing them during different lengths of time. Rats were nose-only exposed for 45 minutes, 90 minutes, 3 hours or 6 hours per day for 4 consecutive days to particle free air, or atmospheres containing either I $8 \mathrm{~nm}, 34 \mathrm{~nm}, 60$ $\mathrm{nm}$ or $\mathrm{I} 60 \mathrm{~nm}$ silver nanoparticles, 3 rats per group. All rats were sacrificed 24 hours after exposure to determine the induction of pulmonary toxicity and the tissue distribution. 
Besides the dose-response data, we investigated whether possible effects were persistent. Per particle size, rats were exposed for 6 hours per day for 4 consecutive days to either clean air or silver nanoparticles, 5 rats per group, and sacrificed 7 days after exposure.

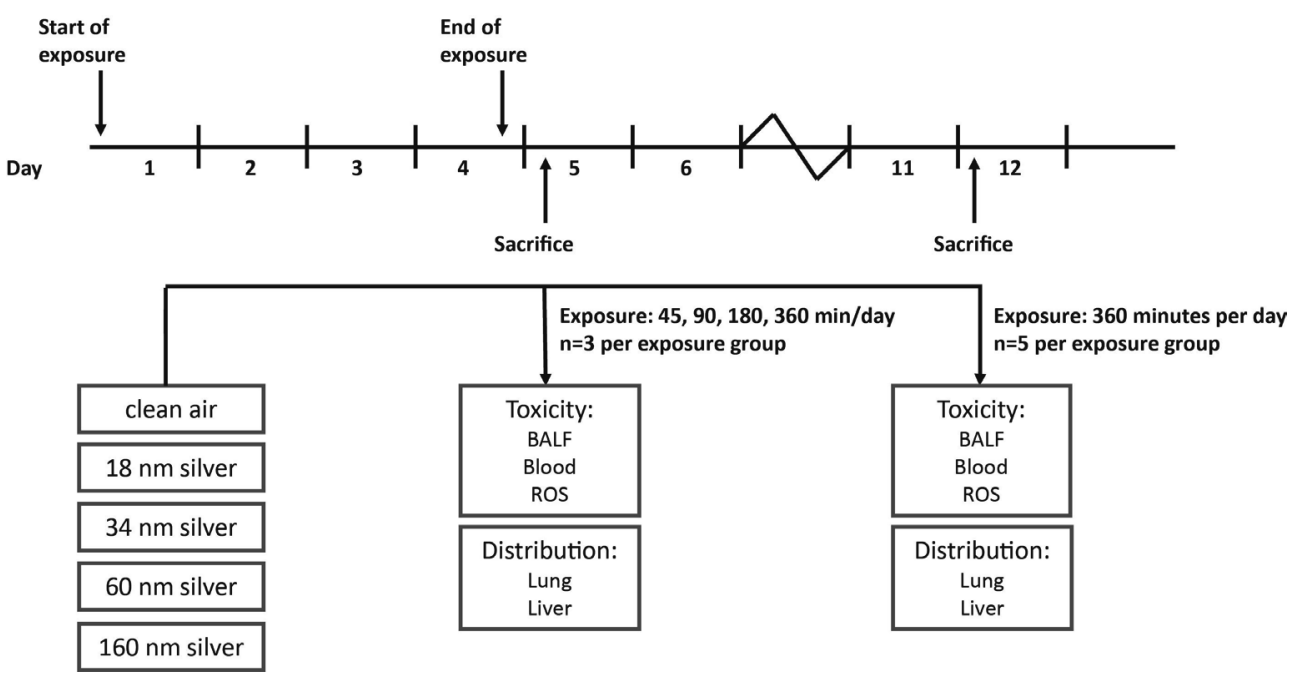

Figure 1. Experimental design of the short-term inhalation study. Rats were nose-only exposed for 45 minutes, 90 minutes, 3 hours or 6 hours per day for 4 consecutive days to particle free air, or atmospheres containing either $18 \mathrm{~nm}, 34 \mathrm{~nm}, 60 \mathrm{~nm}$ or $160 \mathrm{~nm}$ silver nanoparticles, 3 rats per group. All rats were sacrificed 24 hours after exposure to determine the induction of pulmonary toxicity and the tissue distribution. In addition, rats were exposed for 6 hours per day for 4 consecutive days to either clean air or silver nanoparticles, 5 rats per group, and sacrificed 7 days after exposure.

\section{Quantification of silver in tissues by high resolution inductively coupled plasma mass spectrometry}

The presence of silver in the left lung and liver was determined by high resolution inductively coupled plasma mass spectrometer (HR-ICP-MS) (Krystek et al., 20 I3, Krystek, 20 I2). In the other collected organs (spleen, kidneys, testis and brain), we did not measure the level of silver because the amount of silver in these organs was expected to be below the detection limit of $0.0 \mathrm{I} \mu \mathrm{g} / \mathrm{g}$ tissue based on previous finding using $\mathrm{I} 5 \mathrm{~nm}$ and $4 \mathrm{IO}$ $\mathrm{nm}$ silver particles (Brakhuis et al., 20I4a). Samples of homogenized tissue (max. 0.5 g) were completely dissolved on a block heater (Stuart SBH200D, supplied by Omnilabo, Breda, The Netherlands) for 24 hours at $\mathrm{I} 20^{\circ} \mathrm{C}$ after the direct addition of aqua regia ( 0.5 $\mathrm{mL} \mathrm{60 \%} \mathrm{nitric} \mathrm{acid,} \mathrm{ultrapure,} \mathrm{and} \mathrm{I} .5 \mathrm{~mL} \mathrm{30 \%} \mathrm{hydrochloric} \mathrm{acid,} \mathrm{ultrapure;} \mathrm{Merck,}$ Darmstadt, Germany) and afterwards diluted. The silver concentration in the digests was determined using high resolution inductively coupled plasma mass spectroscopy (HR- 
ICP-MS; Element XR, Thermo Fisher Scientific, Bremen, Germany). External calibration curves of the interference-free silver isotopes were used for quantification. Both silver isotopes $\left({ }^{107} \mathrm{Ag}\right.$ and $\left.{ }^{109} \mathrm{Ag}\right)$ were measured in the low-resolution mode. On-line addition and correction with an internal standard (rhodium with the measured isotope ${ }^{103} \mathrm{Rh}$ in the low-resolution mode) was applied as well. The results of ${ }^{107} \mathrm{Ag}$ were reported and the results of ${ }^{109} \mathrm{Ag}$ were used for control. Sample pre-treatment and analysis were carried out in a cleanroom facility class Ioo ooo.

\section{Estimated deposited dose in lungs using multiple path particle dosimetry (MPPD) model}

To estimate the fractions of deposited dose in the head, tracheobronchial, and alveolar region of the rats, the latest online version of the multiple path particle dosimetry model V2.I I (MPPD model) was used (Asgharian et al., 2009, Cassee et al., 2002) (http://www. ara.com/products/mppd.htm). We used the default parameters of the model for rats, i.e., a forced respiratory capacity of $4 \mathrm{ml}$, head volume of $0.42 \mathrm{ml}$, nasal breathing, tidal volume of 2. I ml, and a breathing frequency of I02/min (Anjilvel and Asgharian, I 995, Asgharian et al., 2009, Yeh et al., 1979). The inspiratory fraction was 0.5 , and no pause was entered. Calculations were done using the count median diameter (CMD), geometric SD, the mass concentration, and a density of $10.49 \mathrm{~g} / \mathrm{cm}^{3}$. Calculations included both deposition of the silver nanoparticles in various regions of the respiratory system (extrathoracic, tracheobronchial, and pulmonary region) and the estimated clearance.

\section{Clinical examinations}

The animals were examined once daily throughout the study period. On exposure days, the animals were examined three times per day (before, during and after exposure). The clinical examination included examination of attitude, animal fur, activity level, food and water intake, and faeces and urine production. The body weight of the animals was determined at arrival, at the start of the exposure period and at the day of sacrifice.

\section{Haematology analysis}

Blood samples were collected in K3EDTA-containing tubes for analysis of cell types and concentrations (ADVIA I 20 Hematology Analyzer, Siemens, Deerfield, IL), and in citrate vials for analysis of inflammatory markers in the blood. The level of fibrinogen in the citrate plasma was measured using a rat fibrinogen ELISA kit (GenWay Biotech, Inc., San Diego, USA) according to manufacturer's instructions.

\section{Bronchoalveolar lavage fluid analysis}

The left lung of the rats was bound just below the bifurcation of the trachea and the right lung was cannulated via the trachea. Bronchoalvelar lavage was performed in situ 
by infusing the right lung three times with $27 \mathrm{ml} / \mathrm{kg}$ physiological saline. The retrieved bronchoalveolar lavage fluid (BALF) was kept on ice and centrifuged for Io minutes at $400 \mathrm{~g}$. The pellet was suspended in physiological saline for analysis of the total cell number (Coulter Counter Z2, Coulter Electronics, Mijdrecht, The Netherlands) and cell differentials. Cytospin preparations were stained according to May-Grünwald and Giemsa and evaluated microscopically for macrophages, polynuclear macrophages, neutrophils, lymphocytes, monocytes, and eosinophils. At least 400 cells were counted, 200 cells per slide. The cell-free supernatant was collected to assess cell damage by measurement of total protein content and the release of lactate dehydrogenase (LDH) by Beckman Coulter autoanalyser Unicel DxC80o Clinical Systems (Beckman Coulter, Inc.), and to assess the induction of pro-inflammatory cytokines. The remainder of the supernatant was stored at $-80^{\circ} \mathrm{C}$.

\section{Measurement of pro-inflammatory cytokines}

The presence of pro-inflammatory cytokines IL-I beta, IL-6, TNF-alfa, GM-CSF, MCP-I, ILI 2p70, MIP-2 and RANTES in the supernatant of the BALF was measured by a Bio-Plex Pro assay for rat cytokines according to manufacturer's instructions (Bio-Rad Laboratories, Inc.).

\section{Measurement of oxidative stress}

The induction of oxidative stress was determined by measuring the amount of reduced, oxidized and total glutathione in the lungs. The rinsed right lung was homogenized on ice in $4 \mathrm{ml}$ of Phosphate buffer ( $\mathrm{pH}$ 7.2). Subsequently, the homogenate was centrifuged at $600 \mathrm{~g}$ for Io minutes. To extract the proteins from the samples, the supernatant was mixed I:I with metaphosphorid acid, incubated for I 5 minutes on ice, and centrifuged at $4000 \mathrm{rpm}$ for Io minutes. The amount of reduced, oxidized and total glutathione was measured by Beckmann Coulter Autoanalyser (Beckmand Coulter, Inc.) in the supernatant.

\section{Dose metrics}

The dose-response data was analysed using different dose metrics to determine the most suitable dose metric to describe the effects of silver nanoparticles after inhalation. The exposure was analysed in three different ways: $\mathrm{I}$ ) as the external silver concentration in air, measured during exposure, 2 ) as the internal lung dose of silver, measured by HRICP-MS, and 3) as the alveolar dose, calculated using the exposure concentration and the internal lung dose in combination with the MPPD model. In addition, the data was visualized and analysed based on mass, particle number and total surface area for the external, internal and alveolar dose. In total, nine different graphs were produced and analysed for each chosen effect parameter. In the graphs representing the internal lung and alveolar dose, the dose is different for each animal. 


\section{Statistical analysis}

The raw data of the BALF was corrected for retrieved fluid. All experimental data were combined in one dataset, which was analysed using the dose-response modelling software PROAST (RIVM, Bilthoven, the Netherlands) (Slob, 2002, RIVM, 20I4), according to methods described earlier (Park et al., 2009). PROAST is benchmark dose software that can be used for dose-response analysis (Barlow et al., 2009, EFSA, 2009). Briefly, the PROAST software selects the optimal data fitting model from an exponential family of models using the likelihood-ratio criterion (Slob, 2002). To correct for the different within-group variances between the different exposure groups, the data was log-transformed (Slob, 20 I 4 a, Slob, 20 I 4 b). For the comparison of particles, the particle identification ( 18,34 , 60,160 ) was defined as a covariate, which reflects the potency of the administered agent. A p-value of $<0.05$ for the associated likelihood-ratio test was considered as a statistically significant effect of particle size. The potential differences between particle sizes was further quantified by the critical effect dose of silver nanoparticles $\operatorname{CED}_{20}$, defined as the (nominal) dose resulting in a $20 \%$ response compared to the control group. We chose a critical effect size of $20 \%$ based on the magnitude of effect in previous studies with ambient particulate matter or nanoparticles (Stoeger et al., 2006, Gosens et al., 20I4, Gerlofs-Nijland et al., 2007) and expert judgement on physiologically relevant changes. In addition, the data was analysed by the Kruskal Wallis nonparametric test (Graphpad Prism). Statistical significance is indicated with a ${ }^{*}(p=<0.05)$. In all graphs, error bars represent the standard deviation of the mean.

Table 1. Nanoparticle characteristics. Particle size is given as count median diameter (CMD) with the geometric standard deviation (GSD). Mass, particle number and surface area shown as mean (SD).

\begin{tabular}{|l|l|l|l|}
\hline Agglomerate size $[\mathrm{nm}]$ & Mass $\left[\mu \mathrm{g} / \mathrm{m}^{3}\right]$ & Particle number $\left[\# / \mathrm{cm}^{3}\right]$ & Surface area $\left[\mathrm{nm}^{2} / \mathrm{cm}^{3}\right]$ \\
\hline $18.1(1.24)$ & $438(130)$ & $1.3 \times 10^{7}\left(4.0 \times 10^{6}\right)$ & $1.4 \times 10^{10}\left(4.1 \times 10^{9}\right)$ \\
\hline $34.5(1.3)$ & $325(50)$ & $6.6 \times 10^{6}\left(1.0 \times 10^{5}\right)$ & $5.4 \times 10^{9}\left(9.0 \times 10^{8}\right)$ \\
\hline $60.3(1.32)$ & $341(21)$ & $3.6 \times 10^{5}\left(2.1 \times 10^{4}\right)$ & $3.2 \times 10^{9}\left(1.1 \times 10^{8}\right)$ \\
\hline $160(1.55)$ & $1109(46)$ & $5.3 \times 10^{4}\left(1.7 \times 10^{3}\right)$ & $1.4 \times 10^{9}\left(4.3 \times 10^{8}\right)$ \\
\hline
\end{tabular}

\section{RESULTS}

\section{Nanoparticle characteristics}

The particle characteristics are summarized in table $\mathrm{I}$. The generated particle sizes were I8.I $\mathrm{nm}$ (GSD I.24), $34.5 \mathrm{~nm}$ (GSD I.30), $60.3 \mathrm{~nm}$ (GSD I.32), and I60 nm (GSD I.55) and confirmed by SEM (figure $S_{I}$ ). The $160 \mathrm{~nm}$ particles probably consisted of three to five agglomerated silver nanoparticles of $80 \mathrm{~nm}$ that were fused into single spherical 
particles in the oven. The exposure mass concentrations were similar for the particles of 18,34 and $60 \mathrm{~nm}$ and about 3 times higher for the $160 \mathrm{~nm}$ particles to obtain total particle surface area concentrations in the same range. The particle size distributions and number concentrations are shown in figure $S_{2}$.

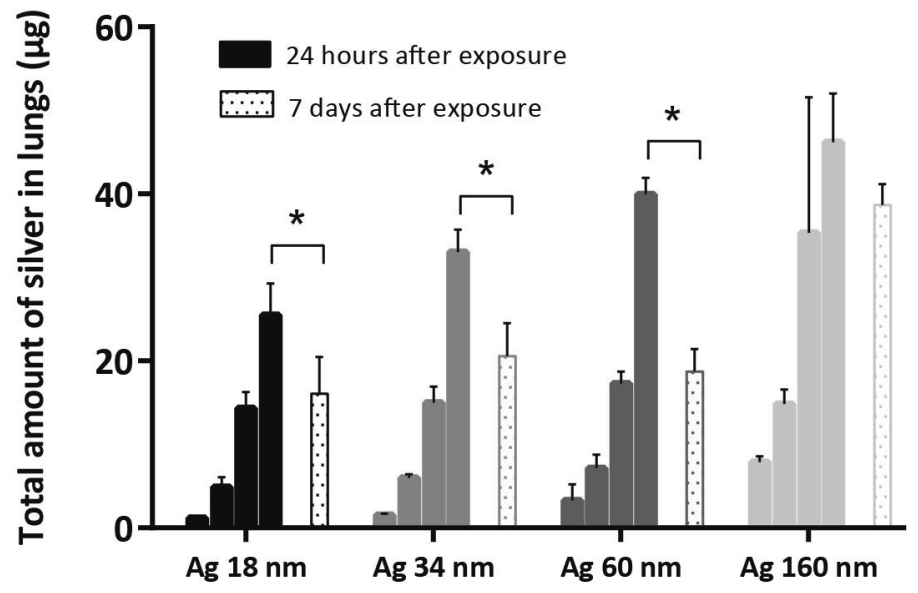

Figure 2. Amount of silver detected in the lungs by HR-ICP-MS at 24 hours and 7 days after exposure. Columns show doses administered from left to right based on control air, and 45 minutes, 90 minutes, 3 hours or 6 hours inhalation exposure to silver nanoparticles per day. At 24 hours after exposure, the level of silver in the lungs correlated with the exposure concentrations, showing a dose-response with increasing total lung deposition upon increasing exposure concentrations. For the $18 \mathrm{~nm}, 34 \mathrm{~nm}$ and $60 \mathrm{~nm}$ particles, the amount of silver in the lungs at 7 days after exposure was significantly lower compared to 24 hours after exposure $(p<0.05, p<0.01, p<0.0001$, respectively).

\section{Quantification of silver in lung and liver by HR-ICP-MS}

In both the lungs and the liver, the level of silver showed an exposure concentration dependent increase at 24 hours after exposure. The level of silver measured in the left lung was normalized to the total lung weight to calculate the total amount of silver in the lungs. For the highest concentration tested, the total lung deposition of silver was $25.5 \mu \mathrm{g}$ of the I $8 \mathrm{~nm}$ particles, $33.0 \mu \mathrm{g}$ of the $34 \mathrm{~nm}$ particles, $40.0 \mu \mathrm{g}$ of the $60 \mathrm{~nm}$ particles, and $46 . \mathrm{I} \mu \mathrm{g}$ of the $160 \mathrm{~nm}$ particles (figure 2). At 7 days after exposure, the amount of silver in the lungs decreased to I6.I $\mu \mathrm{g}$ for the $\mathrm{I} 8 \mathrm{~nm}$ particles, $20.6 \mu \mathrm{g}$ for the $34 \mathrm{~nm}$ particles, I $8.7 \mu \mathrm{g}$ for the $60 \mathrm{~nm}$ particles, and $38.7 \mu \mathrm{g}$ for the $\mathrm{I} 60 \mathrm{~nm}$ particles. The clearance from the lungs was the lowest for the $\mathrm{I} 60 \mathrm{~nm}$ particles; about $\mathrm{I} 6 \%$ of the total mass burden was cleared at 7 days after exposure compared to a clearance of about $37 \%$ for the $\mathrm{I} 8 \mathrm{~nm}$ and $34 \mathrm{~nm}$ particles and even $53 \%$ for the $60 \mathrm{~nm}$ particles. In the liver, the amount of detected silver was $0.52 \mu \mathrm{g}$ for the I $8 \mathrm{~nm}$ particles, $0.75 \mu \mathrm{g}$ for the $34 \mathrm{~nm}$ particles, $0.7 \mathrm{I} \mu \mathrm{g}$ for the $60 \mathrm{~nm}$ particles and 0.24 $\mu \mathrm{g}$ for the $\mathrm{I} 60 \mathrm{~nm}$ particles for the highest concentration tested at 24 hours after exposure. 
Compared to the total lung burden, the relative amount of silver in the liver was the highest for the $34 \mathrm{~nm}$ particles and the lowest for the $160 \mathrm{~nm}$ particles (figure 3). The fraction of total deposited silver in the lungs that reached the liver decreased with increasing total lung burden. At 7 days after exposure, the level of silver in the liver decreased to $0.07 \mu \mathrm{g}, 0.3 \mathrm{I}$ $\mu \mathrm{g}$, $0.17 \mu \mathrm{g}$, and $0.17 \mu \mathrm{g}$ for the $\mathrm{I} 8 \mathrm{~nm}, 34 \mathrm{~nm}, 60 \mathrm{~nm}$, and $\mathrm{I} 60 \mathrm{~nm}$ particles, respectively.
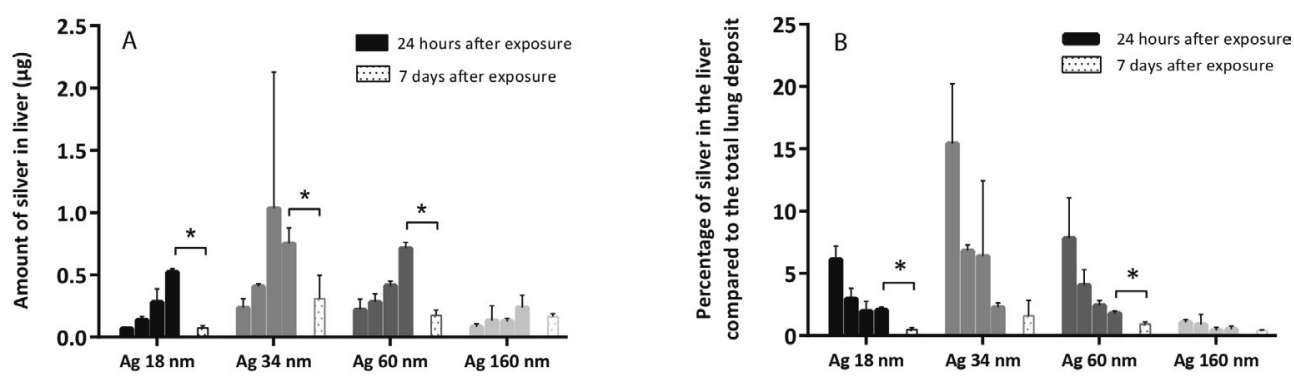

Figure 3. Amount of silver detected in the liver by HR-ICP-MS at 24 hours and 7 days after exposure, expressed as total amount of silver $(A)$ and as percentage of the deposited dose in the lungs (B). The total amount of silver in the liver showed an increase upon increasing exposure concentrations in air (A). For the $18 \mathrm{~nm}, 34 \mathrm{~nm}$, and $60 \mathrm{~nm}$ particles, the total amount of silver in the liver at 7 days after exposure was significantly lower compared to 24 hours after exposure ( $p<0.001$ for all three particle sizes). When expressed as fraction of the deposited dose in the lungs, smaller fractions of silver reach the liver upon increasing deposited doses in the lungs (B). For the $18 \mathrm{~nm}$ and $60 \mathrm{~nm}$ particles, the fraction of silver in the liver at 7 days after exposure was significantly lower compared to 24 hours after exposure ( $p<0.01$ for both particle sizes).

\section{Estimated deposited alveolar dose in lungs using Multiple Path Particle Dosimetry (MPPD) model}

According to the MPPD model, the deposited fraction of the inhaled dose of the silver nanoparticles in the tracheobronchial and alveolar region of the lungs was 0.66 for the I $8 \mathrm{~nm}$ particles, 0.5 for the $34 \mathrm{~nm}$ particles, 0.37 for the $60 \mathrm{~nm}$ particles and 0.39 for the $160 \mathrm{~nm}$ particles. The remaining fraction can be exhaled or may deposit in the nasal cavity. For the alveolar region, the deposition fraction is 0.28 for both the $18 \mathrm{~nm}$ and 34 $\mathrm{nm}$ particles, 0.22 for the $60 \mathrm{~nm}$ particles and o. I o for the $\mathrm{I} 60 \mathrm{~nm}$ particles. Based on the exposure concentration and the HR-ICP-MS results in combination with the MPPD model, we estimated the deposited dose in the alveolar region. Based on mass, the alveolar dose was calculated to be I I $\mu \mathrm{g}$ for the I $8 \mathrm{~nm}$ particles, I $9 \mu \mathrm{g}$ for the $34 \mathrm{~nm}$ particles, $24 \mu \mathrm{g}$ for the $60 \mathrm{~nm}$ particles and $\mathrm{I} 2 \mu \mathrm{g}$ for the $\mathrm{I} 60 \mathrm{~nm}$ particles for the highest concentration tested at 24 hours after exposure (figure 4 ). This equals to a particle number of $2.1 \times 1 \mathrm{O}^{1 \mathrm{I}}$,

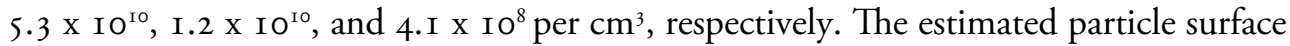
area dose in the alveoli is $2.2 \times \mathrm{IO}^{14} \mathrm{~nm}^{2} / \mathrm{cm}^{3}$, I.9 $\mathrm{x} \mathrm{IO}^{14} \mathrm{~nm}^{2} / \mathrm{cm}^{3}, \mathrm{I} .4 \times \mathrm{IO}^{14} \mathrm{~nm}^{2} / \mathrm{cm}^{3}$, and $3.3 \times \mathrm{IO}^{13} \mathrm{~nm}^{2} / \mathrm{cm}^{3}$ for the $18 \mathrm{~nm}, 34 \mathrm{~nm}, 60 \mathrm{~nm}$, and $\mathrm{I} 60 \mathrm{~nm}$ particles, respectively, 
at the highest concentration tested at 24 hours after exposure. When expressed as mass, the internal alveolar dose is does not differ that much between the different particle sizes. When expressed as particle number or particle surface area, the internal alveolar dose is much higher for the smaller particles compared to the larger particles. For the $\mathrm{I} 8 \mathrm{~nm}$ particles, the particle number in the alveoli is about 5 Io times higher compared to the $\mathrm{I} 60 \mathrm{~nm}$ particles and the particle surface area is about 6.5 times higher.
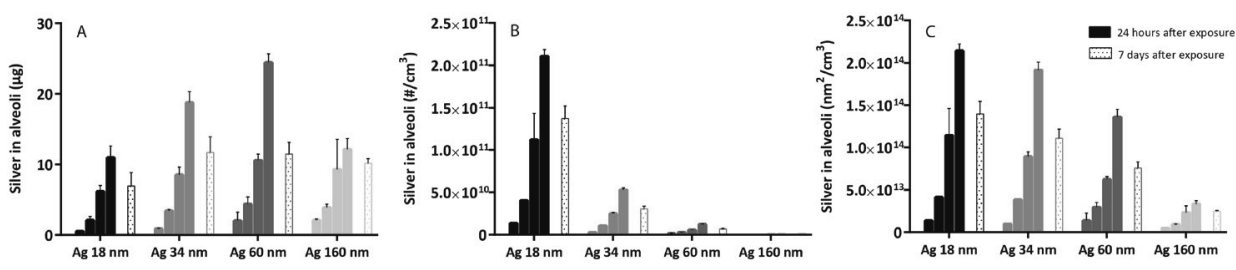

Figure 4. Estimated amount of silver in the alveoli based on the exposure concentrations, HR-ICP-MS measurements and the MPPD model. Results are shown as the estimated mass $(A)$, particle number $(B)$ and particle surface area $(C)$ in the alveoli. Columns show doses administered from left to right based on control air, and 45 minutes, 90 minutes, 3 hours or 6 hours inhalation exposure to silver nanoparticles per day.

\section{Clinical examinations}

Exposure to silver nanoparticles of $\mathrm{I} 8 \mathrm{~nm}, 34 \mathrm{~nm}, 60 \mathrm{~nm}$ and $\mathrm{I} 60 \mathrm{~nm}$ did not induce any premature mortality. There were no effects on animal attitude, fur, activity pattern, food and water consumption and faeces and urine production, and no effects on body weights and organ weights throughout the study (table $S_{I}$ ).

\section{Haematology}

In blood, measured parameters (erythrocytes, leukocytes, leukocyte differentiation, platelets and reticulocytes, haemoglobin, haematocrit, $\mathrm{MCV}, \mathrm{MCH}$ and $\mathrm{MCHC}$ ) did not change at 24 hours or 7 days after exposure to silver nanoparticles (data not shown). There were no differences in the haematological endpoints among the groups of rats exposed to the different particle sizes. In addition, there were no differences detected in the level of fibrinogen in the plasma between the controls and the exposed animals (data not shown).

\section{Total cell counts and differential cell counts in the BALF}

For all particle sizes, the total cell number showed an exposure concentration dependent increase at 24 hours after the end of the exposure period. The increased total cell number was mainly caused by a neutrophil influx (figure $S_{3}$ ). The number of neutrophils 
increased exposure concentration-dependently for all particle sizes. For the $18 \mathrm{~nm}$ and the $34 \mathrm{~nm}$ silver nanoparticles, the number of lymphocytes and monocytes also showed an exposure concentration dependent increase at 24 hours after exposure. For the $60 \mathrm{~nm}$ and $\mathrm{I} 60 \mathrm{~nm}$ particles, there was no increase of lymphocytes and monocytes. The number of macrophages and eosinophils was not affected by the exposure to silver nanoparticles at 24 hours after exposure (data not shown).

At 7 days after exposure, the total cell number decreased for all particle sizes compared to 24 hours after exposure. However, for the animals exposed to $60 \mathrm{~nm}$ silver nanoparticles the total cell number was still significantly increased compared to the controls $(p<0.05)$ (figure $\mathrm{S}_{4}$ ). Although the number of neutrophils decreased at 7 days after exposure compared to 24 hours after exposure, for the animals exposed to $34 \mathrm{~nm}$ and $60 \mathrm{~nm}$ silver nanoparticles the number of neutrophils was still significantly increased compared to the controls ( $p<0.00 \mathrm{I}$ and $p<0.0 \mathrm{I}$, respectively). In addition, the number of lymphocytes in the BALF was significantly increased in the animals exposed to $34 \mathrm{~nm}$ and $60 \mathrm{~nm}$ silver nanoparticles at 7 days after exposure compared to the controls $(p<0.0$ I and $p<0.05$, respectively). There was no significant difference between the exposed groups and the controls in the number of macrophages and monocytes at 7 days after exposure. These results show a prolonged inflammation in the lungs after exposure to $34 \mathrm{~nm}$ and $60 \mathrm{~nm}$ silver nanoparticles.

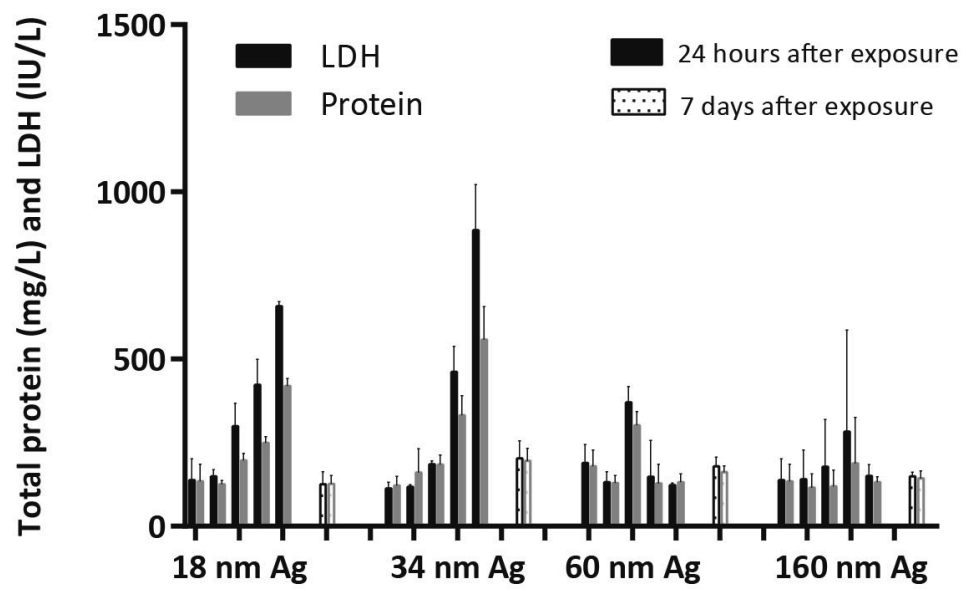

Figure 5. The level of protein and LDH in the BALF at 24 hours and 7 days after exposure to different sizes of silver nanoparticles based on the mass exposure concentrations. Exposure to $18 \mathrm{~nm}$ and $34 \mathrm{~nm}$ silver particles induced an exposure concentration dependent increase in the level of protein and LDH in the BALF, whereas exposure to $60 \mathrm{~nm}$ and $160 \mathrm{~nm}$ silver particles do not. Columns show doses administered from left to right based on control air, and 45 minutes, 90 minutes, 3 hours or 6 hours inhalation exposure to silver nanoparticles per day. 


\section{LDH and total protein in the BALF}

Exposure to $18 \mathrm{~nm}$ and $34 \mathrm{~nm}$ silver nanoparticles induced an exposure concentration dependent increase in LDH and total protein in the BALF, indicating the induction of cellular damage in the lungs at 24 hours after exposure. Exposure to $60 \mathrm{~nm}$ and $\mathrm{I} 60$ $\mathrm{nm}$ silver particles did not induce an increase in LDH and total protein in the BALF at 24 hours after exposure (figure 5). At 7 days after exposure, both the levels of LDH and total protein decreased. The level of $\mathrm{LDH}$ in animals exposed to $34 \mathrm{~nm}$ silver nanoparticles was still significantly increased compared to the controls $(p<0.05)$. The level of total protein did not significantly differ between the controls and the exposed groups at 7 days after exposure.

\section{Pro-inflammatory cytokines in the BALF}

Of the selected cytokines, IL-I $\beta$, TNF- $\alpha$, MCP-I and RANTES showed an exposure concentration dependent increase for all particle sizes. The results of IL-I $\beta$ and MCP-I are shown in figure $S_{5}$. IL-6, IL-I 2 P7O and GM-CSF were below the detection limit. MIP-2 was detectable, but did not correlate with the exposure to silver particles.

\section{Oxidative stress in the lungs}

The induction of oxidative stress was determined by measuring the level of glutathione in the lungs of the rats exposed to silver nanoparticles. The level of total glutathione did not differ between the controls and the exposed groups. In addition, exposure to silver nanoparticles did not induce any significant changes in the level of reduced (GSH) and oxidized (GSSG) glutathione. The ratio of GSH/GssG was also not affected by the exposure to silver nanoparticles (data not shown).

\section{Dose metrics}

We selected the total cell number, the number of neutrophils, and the level of ILI $\beta$ and MCP-I in the lung fluid at 24 hours after the end of the exposure period to determine the most suitable dose metric for silver nanoparticles after inhalation as these were significantly affected by the test atmospheres. The data was analysed using I) the external silver concentration in air as measured during exposure, 2) the internal lung dose of silver as measured by HR-ICP-MS, and 3) the alveolar dose as calculated using the exposure concentration and the internal lung dose in combination with the MPPD model. In addition, the data is graphically presented based on mass, particle number and total surface area for external, internal and alveolar dose. For each of the chosen effect parameters, nine different graphs were produced (figure 6) and they all showed that when the particle surface area that reaches the alveoli is used as a dose metric, the dose-response curves of the various particle sizes almost completely overlap (figure 7). 

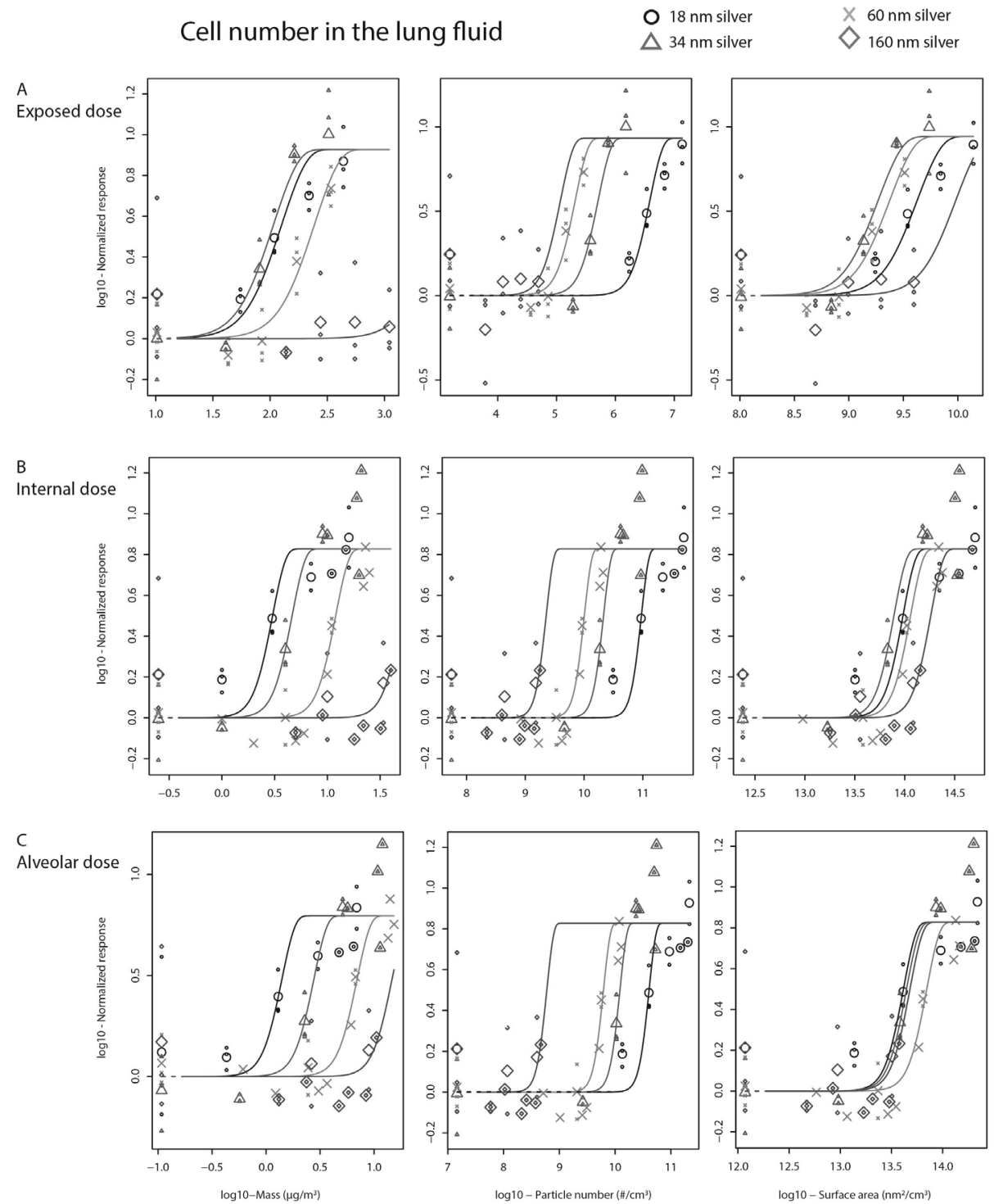

Figure 6. Total cell number in the BALF after inhalation exposure to different sizes of silver nanoparticles. The data is presented based on the measured silver concentration in the exposed air (A), the measured silver amount in the lungs (B), and the estimated silver amount in the alveoli (C). In addition, the data is presented based on mass (left), particle number (middle), and particle surface area (right). The small symbols represent individual animals and the larger symbols represent mean values of animals within the same dosegroup. 

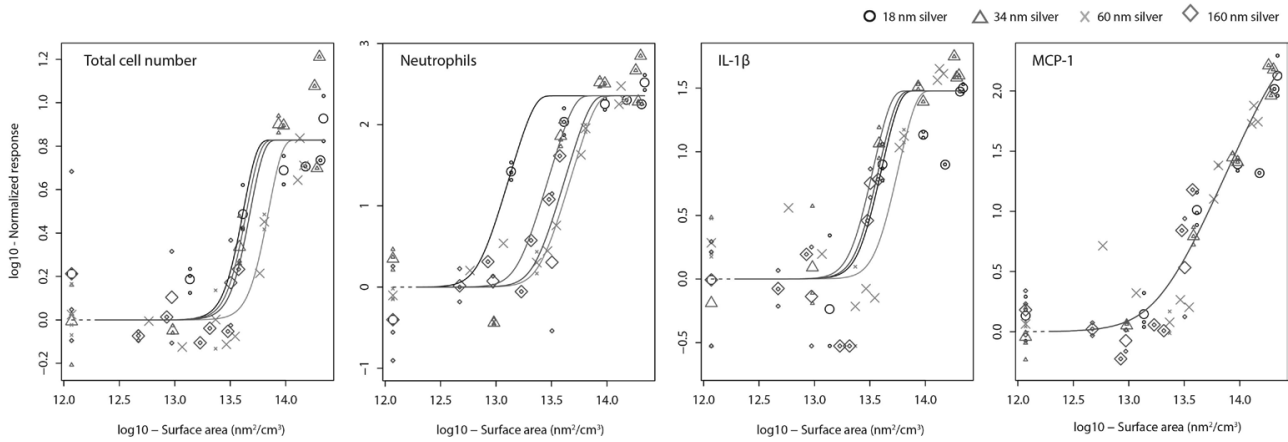

Figure 7. Total cell number, number of neutrophils, IL-1 $\beta$ and MCP-1 in the BALF at 24 hours after inhalation exposure to different sizes of silver nanoparticles based on the particle surface area dose in the alveoli. All particle sizes show overlapping dose-dependent increases in the total cell number, number of neutrophils, IL-1 $\beta$ and MCP-1 in the BALF. The small symbols represent individual animals and the larger symbols represent mean values of animals within the same dose-group.

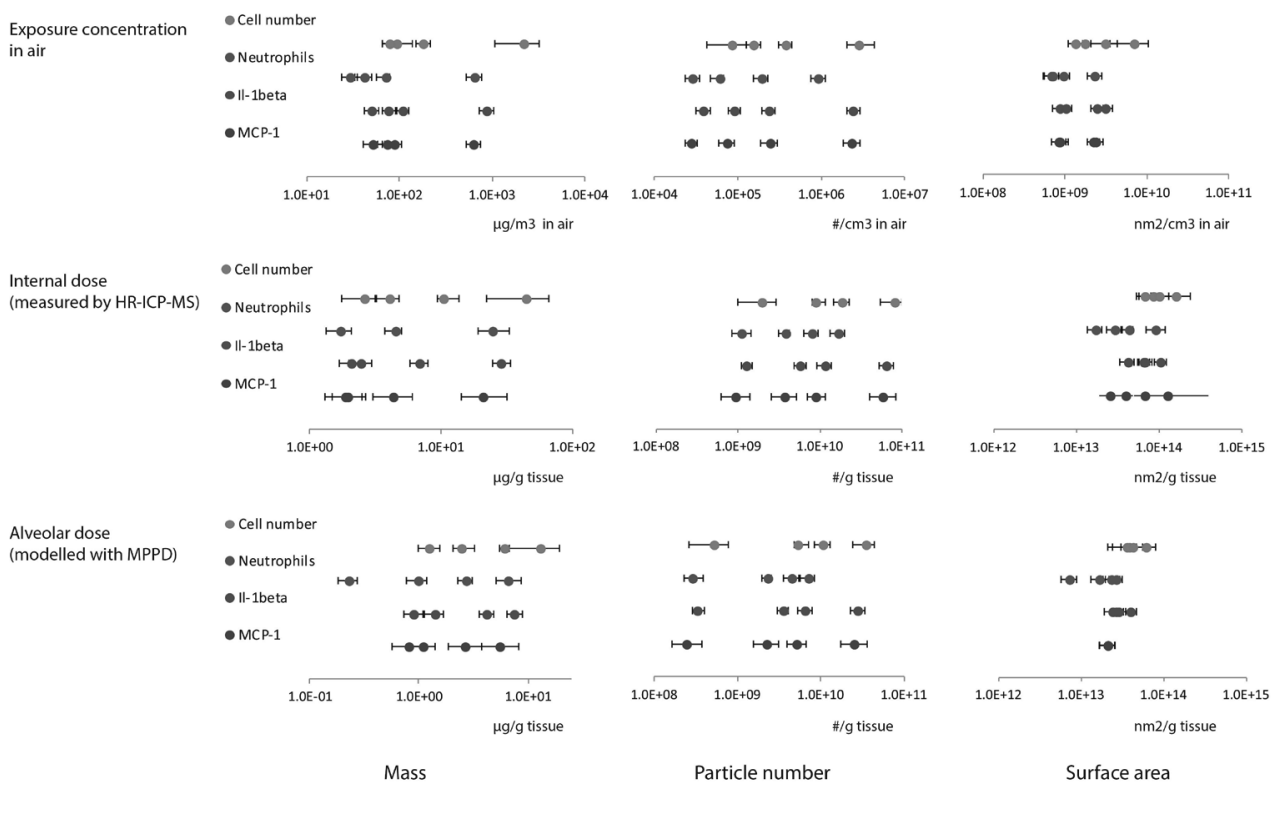

Figure 8. Critical effect doses for silver nanoparticles inducing a $20 \%$ increase in the total cell number, the number of neutrophils and the level of IL-1 $\beta$ and MCP-1 in the bronchoalveolar lavage fluid compared to the controls ( $\mathrm{CED}_{20}$ ). The error bars represent a $95 \%$ confidence interval of the $\mathrm{CED}_{20}$. The figure shows that the $\mathrm{CED}_{20}$ of different sized silver nanoparticles after inhalation is similar when the dose is expressed as the total surface area of the silver nanoparticles that reach the alveoli. 
Using PROAST software, we calculated the critical effect concentration or dose (CED) that would induce a $20 \%$ response compared to the unexposed control animals ( $\mathrm{CED}_{20}$ ) (figure 8). Based on the exposure concentration, the $\mathrm{CED}_{20}$ of the different sized silver nanoparticles do not overlap when expressed as mass or particle number. When expressed as surface area, the critical effect concentrations are more close to each other, but do not overlap. The same happens for the internal dose in the lungs; mass and particle number doses result in different $\mathrm{CED}_{20}$ for the different particle sizes, while surface area brings them more towards each other. Based on the alveolar dose, however, the $\mathrm{CED}_{20}$ of the different particle sizes do overlap when the dose is expressed as surface area (figure 8). Therefore, in the alveoli, particle surface area seems the best dose metric to describe the effect of silver nanoparticles on the total cell number, the number of neutrophils and the level of IL-I $\beta$ and MCP-I after short-term inhalation.

\section{DISCUSSION}

The results of the present study show that after short-term exposure by inhalation the effects of silver nanoparticles (based on a set of markers for inflammation) can best be predicted by the total surface area of the silver nanoparticles that reach the alveoli. There are several reasons why the alveolar dose expressed as particle surface area is a suitable dose descriptor for the pulmonary toxicity of silver nanoparticles. I) The alveoli are the site of the observed toxicity. 2) Silver nanoparticles of different sizes have a different deposition pattern in the lungs, including the alveoli. 3) Silver nanoparticles can release silver ions from their surface, possibly causing the observed effects, as has been reported previously (Pratsinis et al., 2013, Beer et al., 2012, Wang et al., 20I4b, Lubick, 2008, Park et al., 20 Iо). The release of ions increases with decreasing particle size due to their relatively larger surface area.

There are two important points of consideration regarding the interpretation of the results of this study. First, the particle surface area in this study was estimated and not measured. Using Palas spark generation, particles are generated with a size distribution. By using the median particle size to estimate the particle surface area, the estimation introduces some uncertainties. Second, the silver nanoparticles in this study were generated using two different methods to be able to include a larger particle size than $65 \mathrm{~nm}$. The larger particles of $\mathrm{I} 60 \mathrm{~nm}$ were generated by nebulizing a dispersion with PVP coated $80 \mathrm{~nm}$ particles, while the smaller particles of $\mathrm{i} 8 \mathrm{~nm}, 34 \mathrm{~nm}$, and $60 \mathrm{~nm}$ were spark generated. As explained in the methods section, the larger particles were led through an oven which removed the PVP coating (Peniche et al., I993, Du et al., 2006) and melted the particles into the single spherical particles depicted in figure $S_{I}$. However, the difference in generation method might have influenced the chemical composition of the nanoparticles by for example differences in the oxidation of the particles. Silver can oxidize in air, although at a slow rate. We believe it is unlikely that silver oxide was formed in the Palas spark generation setup, but we cannot exclude the possibility. There was no oxygen present at the position 
of the electrodes, it was added later on. In addition, at temperatures above $195^{\circ} \mathrm{C}$, the silver oxidation is reversed and silver oxide splits up in silver and oxygen (Franek, 200I), and the temperature of the oven was $700{ }^{\circ} \mathrm{C}$. Therefore, we assume the particles were all similar apart from their diameter. Even though we estimated the particle surface area and we used two different generation methods to produce silver nanoparticles of 4 different sizes, the results of all the different sizes (including the largest particle size) show that the effects of silver nanoparticles after short-term inhalation can best be predicted by their total surface area that reaches the alveoli.

The data presented here confirms previous reports in which the total administered surface area appeared to be the best predictor for particles with the same chemical composition but of different size (Oberdorster et al., 2000, Stoeger et al., 2006, Duffin et al., 2007, Horie et al., 20I2, Kobayashi et al., 2009, Braakhuis et al., 20I4b, Ho et al., 20II). These papers show some evidence that based on the exposed dose; surface area might be a better dose metric to describe the effect of a range of nanoparticles, with the same chemical composition but differing in size, compared to mass. However, they did not take the internal dose in the lungs into account. For intratracheal instillation, one could assume the total bolus reaches the lungs, but in nose-only or whole-body chamber inhalation, the internal dose in the lungs should be measured. It should be noted that high dose rate methods such as intratracheal instillation, elicit significantly greater inflammation compared to low dose rate delivery such as nose-only or whole body inhalation (Baisch et al., 20I4). In the present study, based on the exposure concentration, surface area does bring the dose-response curves of the different particle sizes more towards each other, but the alveolar dose gives almost completely overlapping dose-response curves when surface area as a dose metric is used (figure 7), showing the importance of using the alveolar dose instead of the external exposure concentrations.

To confirm whether the estimated alveolar dose expressed as surface area is a suitable dose descriptor also in other studies, we compared the results of the present study with the results of a previous short-term inhalation study with silver nanoparticles (Braakhuis et al., 20I4a). In the previous study, I $5 \mathrm{~nm}$ silver nanoparticles induced a doubling in the total cell number, a 175 -fold increase in the number of neutrophils, and a 5 -fold increase in the level of IL-I $\beta$ and MCP-I at 24 hours after the end of the exposure period (Braakhuis et al., 20I4a). The mass exposure concentration was measured at $179 \mu \mathrm{g} / \mathrm{m}^{3}$ and the estimated alveolar particle surface area dose was $5.8 \times \mathrm{IO}^{\mathrm{I2}} \mathrm{nm}^{2} / \mathrm{cm}^{3}$ particles per $\mathrm{mm}^{3}$ in the lungs. When compared to the results of the present study, the $18 \mathrm{~nm}, 34$ $\mathrm{nm}, 60 \mathrm{~nm}$, and $\mathrm{I} 60 \mathrm{~nm}$ particles induced these effects at mass exposure concentrations of I I 5, 9I, I 26, and >I IOO $\mu \mathrm{g} / \mathrm{m}^{3}$, respectively, and at alveolar doses of $5.4 \times \mathrm{IO}^{\mathrm{I2}} \mathrm{nm}^{2} /$ $\mathrm{cm}^{3}$ particles per $\mathrm{mm}^{3}$ in the lungs (ranging from $5.0-5.8 \times \mathrm{IO}^{12} \mathrm{~nm}^{2} / \mathrm{cm}^{3}$ ). The alveolar doses expressed as particle surface area are similar in both studies, confirming that this is a suitable dose metric. Therefore, we recommend that internal alveolar doses are used in studies aimed at finding suitable dose metrics. 
We measured the amount of silver in the lungs at 24 hours and 7 days after the end of the exposure period. Probably, there was some clearance from the lung of the (nano) particles between the end of exposure and the sacrifice at 24 hours after exposure and after each day of exposure. According to literature, single nanoparticles and agglomerates of $<\mathrm{IOO} \mathrm{nm}$ are less efficiently phagocytized by alveolar macrophages compared to microparticles or large agglomerates of $>\mathrm{I} \mu \mathrm{m}$ (Bakand et al., 20 I2, Muhlfeld et al., 2008a, Oberdorster and Pott, I987, Phalen et al., 2010) and less efficiently cleared by mucociliary clearance (Geiser and Kreyling, 2010, Geiser et al., 2008, Oberdorster and Pott, 1987). Our results show similar amounts of silver (based on mass) in the lungs at 24 hours after exposure to all four particle sizes. When we compare the amount of silver at 7 days after exposure to the amount at 24 hours after exposure, we can calculate the clearance rate for the different particle sizes. In contrast to the literature that reports slower clearance of poorly soluble nanoparticles compared to larger particles, the clearance from the lungs was the lowest for the $\mathrm{I} 60 \mathrm{~nm}$ particles, i.e. the largest particles in our experiments. This discrepancy might be explained by a) increased clearance from the lungs of the smaller particle sizes by translocation and b) by (size/surface area dependent) dissolution of silver nanoparticles into ions that are more easily cleared compared to particles. The larger particles were generated by nebulizing a dispersion with PVP coated $80 \mathrm{~nm}$ particles, while the smaller particles were spark generated. One could argue that this may account for some of the differences found in the effects between the particles. However, all particles were led through an oven which removed the PVP coating (Peniche et al., I993, Du et al., 2006) and melted the particles into the single spherical particles depicted in figure $S_{\mathrm{I}}$. We assume therefore that the particles were all similar apart from their diameter. The difference in diameter will influence the dissolution rate of the silver particles since the dissolution rate and release of ions is proportional to the diameter of the particles (Ma et al., 20I2). We also noted a size dependent translocation of silver into the liver, with the smallest amounts for the largest particles. In the present study, the amount of silver in the liver decreased at 7 days after exposure compared to 24 hours after exposure, indicating that silver is cleared from the liver and probably only accumulates to a limited extend, depending on the frequency of exposure. The potency of silver to accumulate in organs or tissues of the body should be investigated in experiments using long-term exposure to low concentrations of silver nanoparticles, resembling realistic exposure scenarios for humans.

The present study focused on the short-term inhalation of silver (nano)particles and showed that alveolar dose expressed as particle surface area seems the most suitable dose descriptor for the acute effects of silver nanoparticles across a range of sizes. For the risk assessment of silver nanoparticles, this dose metric can be used to predict the acute effects of comparable, uncoated silver nanoparticles that not have been tested in inhalation studies. Regarding long-term inhalation or chronic effects, the dose descriptor might be different since the kinetics of the silver nanoparticles and dissolution behaviour will play a role in the distribution of silver to other organs or tissues of the body where they might induce toxicity that is not directly correlated to the internal alveolar dose. For 
nanoparticles that differ from the silver nanoparticles tested in this study, for example other chemical composition, shape or coating, the most suitable dose descriptor can be different because of differences in kinetics and mechanisms that might induce toxicity. In other words, it needs to be determined to what extend this results can be generalized.

Here, the dose descriptor was identified by distinguishing kinetics and toxicity. First, the particles that reach the effect site, i.e. the alveoli, were estimated. Subsequently, effects were related to different dose metrics of the alveolar dose. It may be that this approach is much broader applicable: some particle characteristics may have a major impact on kinetic processes, whereas other particle characteristics are important for inducing an effect. As a consequence, when the dose metric is investigated in view of external dose, different findings may occur, based on the interaction between kinetics and toxicity. Distinguishing kinetics and toxicity by determination of the particle characteristics at the effect site may help in understanding the mechanisms underlying a suitable dose metric, and thus aid in understanding and applying such information.

\section{CONCLUSION}

According to the results of the present study, the most suitable dose metric to describe the effect of silver nanoparticles after short-term inhalation is the surface area dose that reaches the alveoli. This dose metric can be used in the risk assessment of silver nanoparticles to predict the acute effects of silver nanoparticles with unknown pulmonary toxicity and to derive exposure limits.

\section{ACKNOWLEDGEMENTS}

This work was supported by the project 'Integrated Risk Assessment of Nanomaterials' from the National Institute for Public Health and the Environment and by the NanoNextnl program 'Risk Analysis and Technology Assessment: Human Health Risks'. We would like to thank Ilse Gosens and John Boere for their help with the experimental design and Harry van Steeg for critical review of the manuscript. We thank Piet K. Beekhof for the analysis of the total protein, LDH and glutathione, and Henny W. Verharen, Hans J.C. Strootman, Ron F. Vlug, Christine M.R. Soputan, Jan Bos, Jolanda Rigters for their excellent technical assistance. In addition, we thank Jose van den Dungen for assistance with sample pretreatments and Chris T. W. M. Schneijdenberg for the assistance with the sEM imaging. 

Adapted from: Progress and future of in vitro models to study translocation of nanoparticles

Hedwig M. Braakhuis ${ }^{\mathrm{I}, 2}$, Samantha Kloet ${ }^{3}$, Sanja Kezic ${ }^{4}$, Frieke Kuper ${ }^{5}$, Margriet V.D.Z. Park ${ }^{2}$, Susann Belmann', Meike van der Zande ${ }^{6}$, Severine Le Gac ${ }^{7}$, Petra Krystek ${ }^{8}$, Ruud Peters ${ }^{6}$, Ivonne Rietjens ${ }^{3}$, Hans Bouwmeester ${ }^{6}$

Archives of Toxicology 2015 Sep; 89(9): 1469-95

Affiliation

I Department of Toxicogenomics, Maastricht University, po Box 6I6, 6200 MD Maastricht, the Netherlands

${ }^{2}$ Centre for Health Protection, National Institute for Public Health and the Environment (RIVM), Po Box I, 3720 BA Bilthoven, the Netherlands

${ }^{3}$ Division of Toxicology, Wageningen University, Tuinlaan 5, 6703 HE Wageningen, the Netherlands

${ }_{4}^{4}$ AMC, Coronel Institute of Occupational Health, Academic Medical Centre, University of Amsterdam, i I05 AZ Amsterdam, the Netherlands

5 TNO, Utrechtseweg 48, 3704 HE Zeist, the Netherlands

${ }^{6}$ RIKILT- Wageningen UR, PO Box 230, 6700 AE Wageningen, the Netherlands

${ }^{7}$ Ut BIOs, Lab on a Chip Group, MESA+ Institute for Nanotechnology, University of Twente, the Netherlands

${ }^{8}$ Philips Innovation Services, High Tech Campus I I, 5656 AE Eindhoven, the Netherlands 


\section{Progress and future of in vitro lung models to study translocation of nanoparticles}




\section{ABSTRACT}

The increasing use of nanoparticles in products likely results in increased exposure of both workers and consumers. Because of their small size, there are concerns that nanoparticles unintentionally cross the barriers of the human body. Several in vivo rodent studies show that, dependent on the exposure route, time, and concentration, and nanoparticle characteristics, nanoparticles can cross the lung barrier. This review aims to evaluate the performance of in vitro lung models that mimic the lung-blood barrier of the human body. In vitro models of varying complexity are available, ranging from single cell type monolayer to multi cell (3D) models. Only a few studies are available that allow comparison of the in vitro translocation to in vivo data. This situation could change since the availability of analytical detection techniques is no longer a limiting factor for this comparison. We conclude that to further develop in vitro models to be used in risk assessment, the current strategy to improve the models to more closely mimic the human situation by using co-cultures of different cell types and microfluidics approaches, are essential. At the current state-of-the-art the in vitro models do not yet allow prediction of absolute transfer rates but they do support the definition of relative transfer rates and can thus help to reduce animal testing by setting priorities for subsequent in vivo testing.

\section{GENERAL INTRODUCTION}

Nanoparticles (NPS) have attractive and novel properties compared to their bulk counterparts and are therefore used in an increasing number of consumer products (Nanotechnologies, 20I4). Examples are zinc oxide and titanium dioxide nanoparticles in sunscreens and silver nanoparticles in food packaging material, textiles, and cosmetics, but many more have been identified (Bouwmeester et al., 2014). The increasing use of nanoparticles in products likely results in increasing exposure of both workers and consumers. Because of the unique properties of nanoparticles that are related to their small size, concerns arise that nanoparticles would unintentionally cross the barriers of the human body, which would result in internal exposure to NPs potentially leading to adverse effects. Several in vivo studies have been performed to assess the distribution of NPs after inhalation (Braakhuis et al., 20 I 4a, Creutzenberg et al., 20I2, Elder et al., 2006, Geraets et al., 20 I 2, Kreyling et al., 2002, Kreyling et al., 2009, Ma-Hock et al., 20 I 2, Oberdorster et al., 2004, Sadauskas et al., 2009, Semmler et al., 2004, Takenaka et al., 200I). They show that, depending on the exposure time and concentration, as well as on their characteristics, NPS can cross the lung barrier.

Information on the kinetics of NPs in the human body is essential for risk assessment purposes, because of their potency to accumulate with normal use. The overall resultant of absorption, distribution, metabolism and excretion (ADME), i.e. internal exposure, will determine target tissue doses and will be critical for the ultimate systemic adverse 
health effects (Geraets et al., 20I4). The current risk assessment of NPs (and chemicals) mainly relies on in vivo studies using animal models (EFSA, 20I I). Whilst these in vivo studies provide unique information on the distribution of NPs in a whole organism, the number of animal studies should be reduced as much as possible for several reasons (Hartung et al., 2013). First, the use of animals is ethically debatable. Secondly, animal models do not fully simulate the physiology of humans. Lastly, given the great number of different and the variety in NPs it is impossible and economically not feasible to test all of them through in vivo studies. Therefore, in vitro models have been developed to study the translocation of NPS (Hartung et al., 2013), which can be used to assess their internal exposure. However, before such in vitro models can reliably be used in risk assessment of NPs, they need to be well described and validated (Kandarova and Letasiova, 20I I, Worth and Balls, 2004) using in vivo data (Genschow et al., 2002).

To obtain reliable NP kinetics data from in vitro or in vivo studies, robust analytical detection methods should be used in the experiments. Over the last couple of years the quality of NP characterization methods used in kinetic studies has been improved, but much is to be gained here. Therefore, we reviewed the current state of the knowledge on analytical detection methods and propose directions for further improvement and incorporation in in vitro or in vivo studies.

In this review we aimed to evaluate the existence and performance of in vitro models that mimic the lung barrier. Where possible we compare the observed translocation in vitro to the in vivo translocation to validate to what extent the in vitro results mimic the in vivo situation. We first describe the different lung in vitro models that are currently in use to study the transfer of NPs via inhalation. In vitro models that are used only to assess the toxicity of NPs, but did not measure translocation, are excluded from this review. After defining the in vitro models, we compare the results of the in vitro models with available in vivo data and discuss their predictive value. Finally, we give recommendations for the future development of relevant in vitro models.

\section{Introduction to the lung barrier}

The main function of the lungs is to transport oxygen from the atmosphere into the bloodstream and to release carbon dioxide from the bloodstream into the atmosphere. During inhalation, air travels from the mouth or nose through the nasopharynx, oropharynx, larynx and trachea. The trachea divides into two main bronchi, which branch to the left and right lungs and subsequently subdivide into a system of bronchi and bronchioles until the alveoli where the gas-exchange takes place. The airways are lined by ciliated respiratory epithelium, which is covered by a mucus layer. The mucociliary movement is an important clearance mechanism, especially to remove inhaled (nano) particles. Deeper in the airways, the clearance is slower, given the increased pathway length and decreased mucous velocity (Geiser and Kreyling, 2010). Nanoparticles, especially soluble ones like $\mathrm{ZnO}$, may be able to translocate the mucus layer and reach the 
epithelial cells and thus cause local damage (Vandebriel and De Jong, 201 2, Landsiedel et al., 20I4a, Frieke Kuper et al., 2015). They may also be able to cross the epithelial barrier and reach underlying interstitium with its blood and lymph vasculature.

The alveoli are lined by a single epithelial layer under which is an interstitium with extracellular matrix, blood capillaries, and stromal cells. The epithelial layer is covered by surfactant at the alveolar luminal side. Alveolar type I cells form the structure of the alveolar wall, these cells are very thin to improve the gas-exchange. In addition, type I cells have tight junctions to prevent chemicals and particles from entering the bloodstream. Besides type I cells, alveolar type II cells secrete pulmonary surfactant to lower the surface tension. Finally, for clearance of particles and pathogens from the lungs, alveolar macrophages are present (Möller et al., 20 Io, Klein et al., 20 I I).

To cross the lung-blood barrier, NPs must deposit in the alveolar region. The deposition of inhaled particles depends on the morphology of the lungs, the respiratory conditions, and the physicochemical properties of the particles. The most important physicochemical properties of inhaled particles that influence deposition are (agglomerate) size, size distribution, density, shape, charge, and hygroscopicity (Pilcer and Amighi, 2010, Carvalho et al., 20II, Braakhuis et al., 2014b). When the agglomerate size of NPs is $<$ IOO $\mathrm{nm}$ but >IO nm, a considerable part will deposit in the alveolar region (about $30 \%$ of the particles) (Oberdorster, I989, ICRP, I994, Asgharian et al., 2009). Below $30 \mathrm{~nm}$, the deposition shifts from the alveoli more towards to tracheobronchial region (Braakhuis et al., 2014b).

Once deposited in the alveoli, NPs can be cleared from the lungs by alveolar macrophages. However, single NPS and agglomerates of $<$ IOO nm are less efficiently phagocytized by alveolar macrophages compared to microparticles or large agglomerates of $>\mathrm{I} \mu \mathrm{m}$ (Bakand et al., 20I2, Muhlfeld et al., 2008a, Phalen et al., 20I0). After uptake of the particles, macrophages can move gradually upward by the mucociliary escalator and are subsequently swallowed and enter the gastrointestinal tract. If not cleared by phagocytosis, NPs can be taken up by the alveolar epithelium and reach pulmonary interstitium from which they are transported to the local lymph nodes, or reach the blood circulation (Borm et al., 2006). Translocated particles may subsequently reach organs where they can be taken up, and might cause damage (Braakhuis et al., 20I4b).

Overview of currently used in vitro lung models to study translocation of nanoparticles Many different in vitro models have been developed to study the translocation of nanoparticles. Most in vitro barrier models culture cells on Transwell ${ }^{\circ}$ inserts, which consist of a permeable membrane separating an apical and a basolateral compartment. Transwells can be used to quantify both uptake of nanoparticles into the cells from the apical compartment and efflux from the cells to the basolateral compartment as a measure of translocation (figure I). Fluorescent polystyrene NPs are the most commonly employed because of their easy detection. 


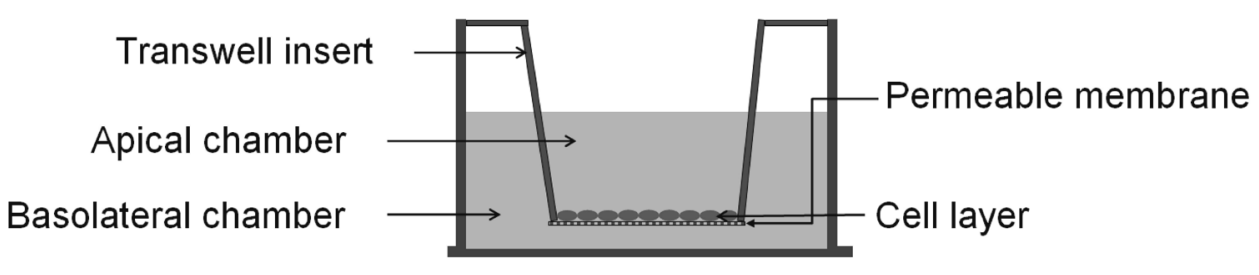

Figure 1. The two-compartment cell culture system contains a permeable cell culture insert, separating two compartments in a Transwell ${ }^{\circledR}$. Cells are seeded and cultured on the inserts to form a barrier between the two compartments.

Table I presents an overview of in vitro barrier models currently used to study the translocation of NPs after inhalation. The most frequently used lung epithelial cells lines are A549, CALU-3, H44I and I6HBei40- (I6HBe). Of these cell lines, CALU-3, H44I and I6HBE form tight junctions, but A549 do not (Lehmann et al., 20I I, Chowdhury et al., 2010, Geys et al., 2006, Hermanns et al., 2004, George et al., 2015). In in vitro translocation studies, primary rat alveolar cells have also been frequently used (Fazlollahi et al., 20 I I, Geys et al., 2009, Yacobi et al., 2008).

Besides differences in cell types, in vitro lung barrier models differ in whether they are submerged or cultured at the air-liquid interface. Submerged models have the advantage of being technically simple. However, the culture medium can alter the properties of the NPs, and subsequently their uptake and effects. Air-liquid models mimic more realistically the inhalation exposure, therefore many air-liquid models have been developed recently (Blank et al., 2006, Brandenberger et al., 20 Iob, Frohlich et al., 2013, Herzog et al., 2013, Holder and Marr, 2013, Lenz et al., 2013, Lenz et al., 2009, Raemy et al., 201 I, Rothen-Rutishauser et al., 2009, Savi et al., 2008, Xie et al., 2012). Disadvantages are the complexity of the system to maintain constant temperature and humidification, and the high costs in comparison with submerged models.

In recent years, co-culture models containing more than one cell type are used to mimic the lung barrier more closely compared to mono-cultures (Klein et al. $20 \mathrm{I} \mathrm{I}$ ). Most models use lung epithelial cells as a basis, those being either primary cells or immortalized cell lines. To obtain co-culture models, different cell types are added to the basic model. The first type of co-culture models include endothelial cells to mimic the alveolar-capillary barrier (Bermudez et al. 2002; Birkness et al. 1995; Chowdhury et al. 2010; Hermanns et al. 20 I0; Hermanns et al. 2004; Papritz et al. 2010). This type of model can be extended by the addition of alveolar macrophages, mast cells, and/or type II alveolar cells. The second type of models does not include endothelial cells, but add dendritic cells and macrophages to the epithelial cell layer (Brandenberger et al. 20 Io; Muller et al. 20io; Rothen-Rutishauser et al. 2008; Rothen-Rutishauser et al. 2005). This type of model can also be completed with type II alveolar cells. The third type of models includes fibroblasts instead of endothelial cells, which can be extended by adding dendritic cells. 
A limited number of NPs have been studied to test translocation across the in vitro lung barrier. These include polystyrene NPs, titanium dioxide NPs, quantum dots, cerium oxide NPS, gold NPS, silica NPs, diesel particles and single-walled carbon nanotubes (table I). The translocation rate of NPS is higher in models with cells that do not form tight junctions compared to cells that do (Geys et al., 2006, Hermanns et al., 2004, George et al., 2015). Probably, the higher translocation rate is a consequence of paracellular transport, which will not occur in a healthy lung but might occur in a damaged or inflamed lung. The pore size of the permeable membrane of the inserts also influences the translocation rate of NPs; the larger the pore size, the higher the translocation rate (Geys et al., 2006).

Ex vivo tissues as model for the lung are not addressed here. The precision cut lung slices (PCLS) taken from human and rodent lungs have been used to study translocation and toxicity of nanomaterials. The use of pCLs for translocation of nanoparticle-mediated drug delivery is recently reviewed by Paranje and Mueller-Goymann (Paranjpe and Muller-Goymann, 20I4). 
Table 1. Overview of in vitro lung models used to study translocation of nanoparticles.

\begin{tabular}{|c|c|c|c|c|c|c|c|c|}
\hline 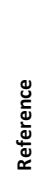 & 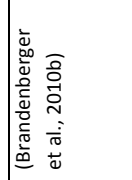 & 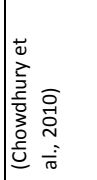 & 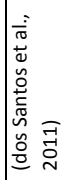 & 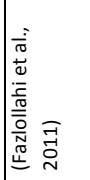 & 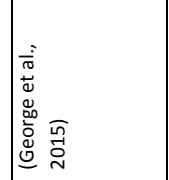 & 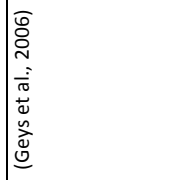 & 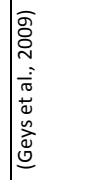 & 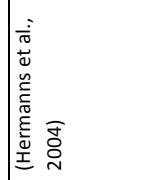 \\
\hline 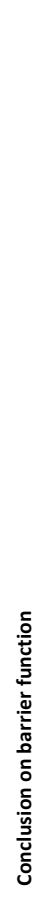 & 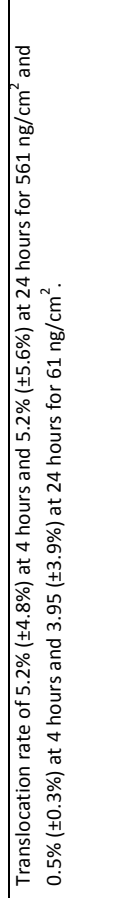 & 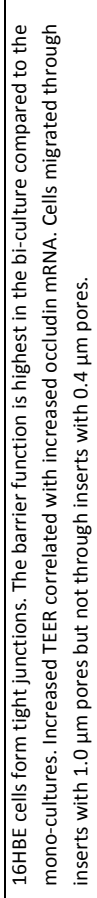 & 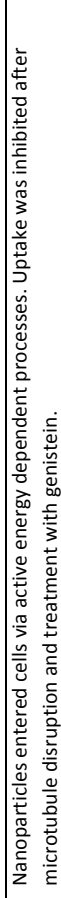 & 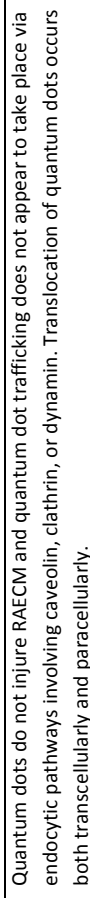 & 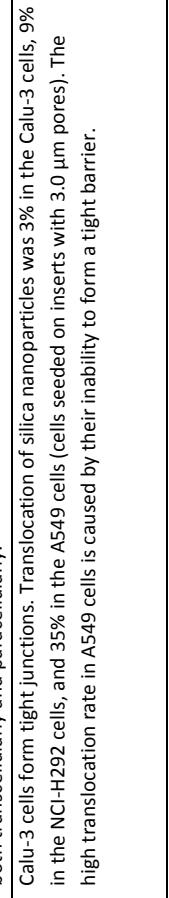 & 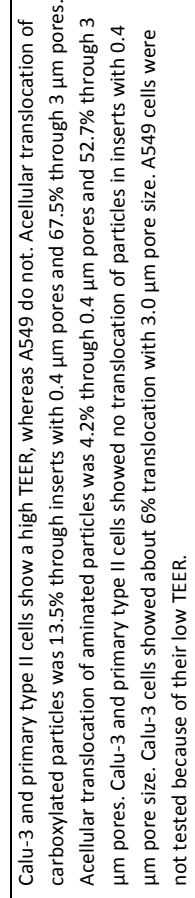 & 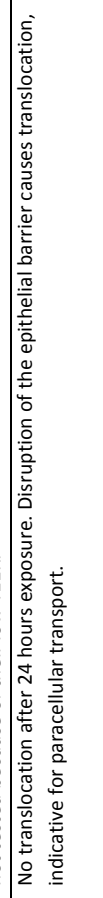 & 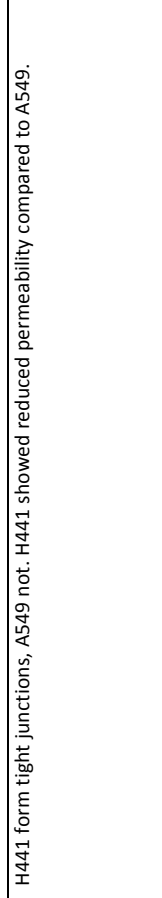 \\
\hline & 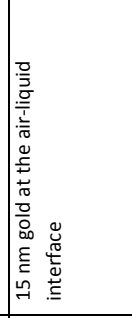 & 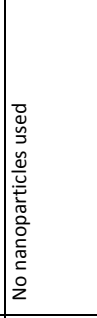 & 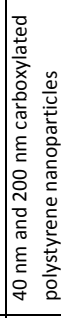 & 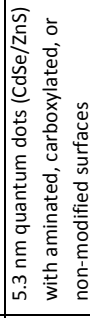 & 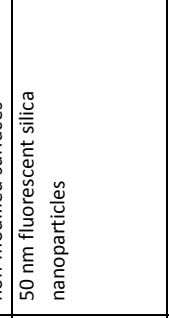 & 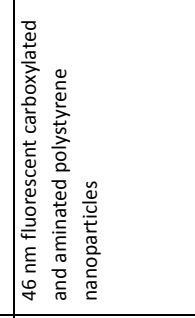 & 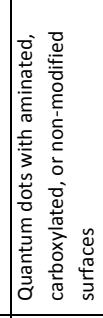 & 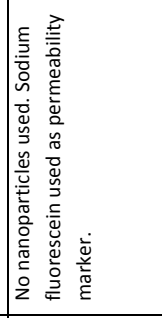 \\
\hline 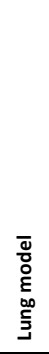 & 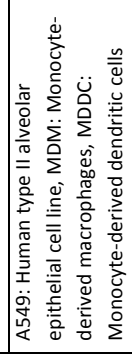 & 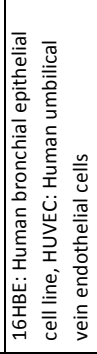 & 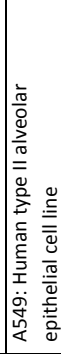 & 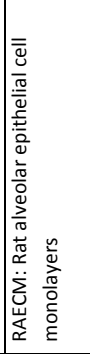 & 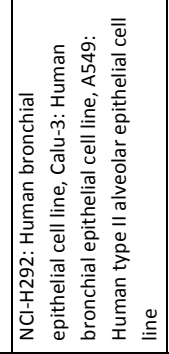 & 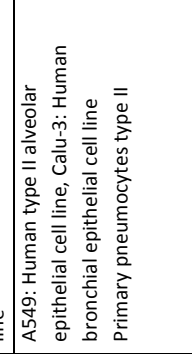 & 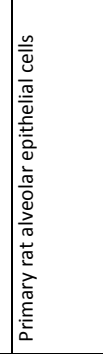 & 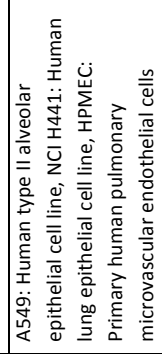 \\
\hline
\end{tabular}




\begin{tabular}{|c|c|c|c|c|c|c|}
\hline & 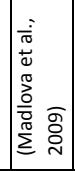 & 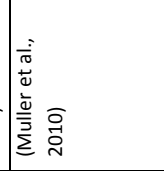 & 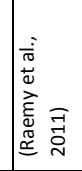 & 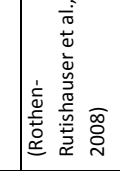 & 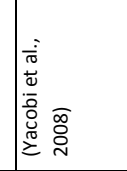 & 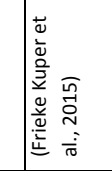 \\
\hline 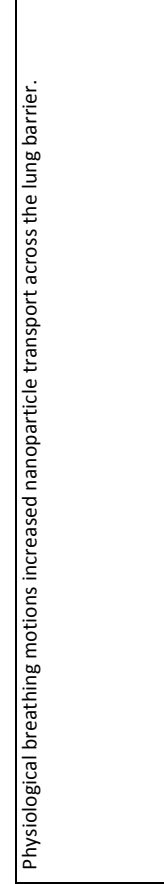 & 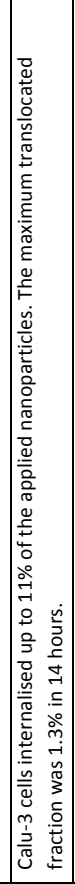 & 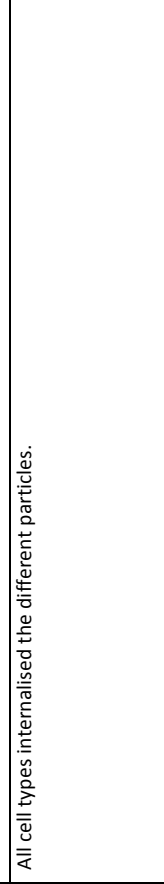 & 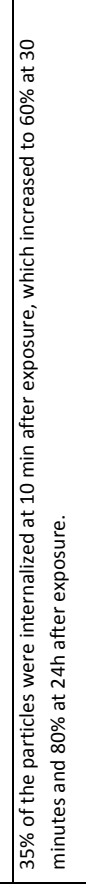 & 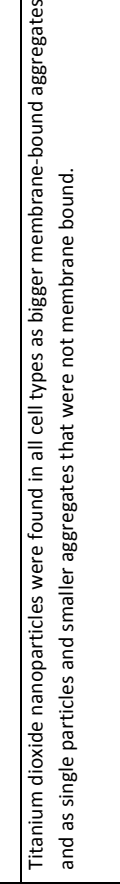 & 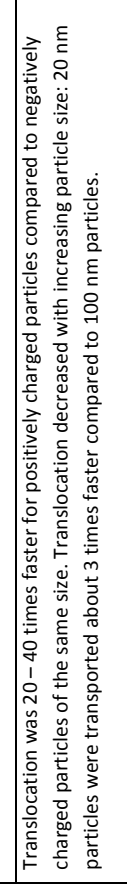 & 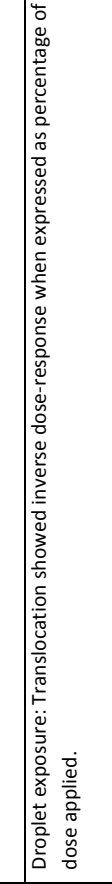 \\
\hline 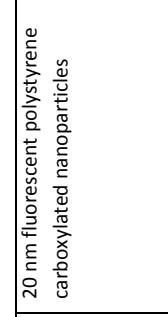 & 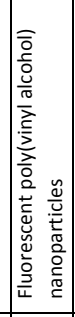 & 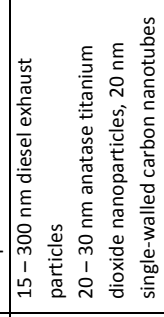 & & 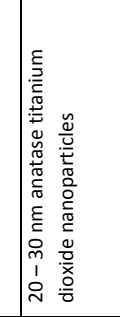 & 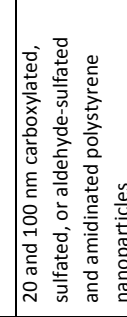 & 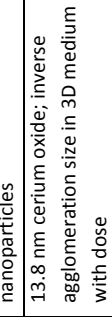 \\
\hline 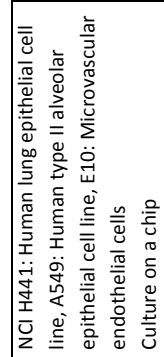 & 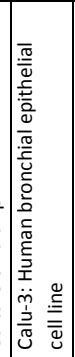 & 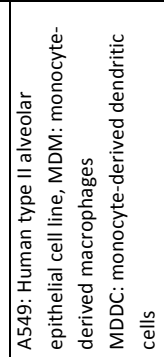 & 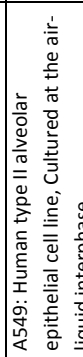 & 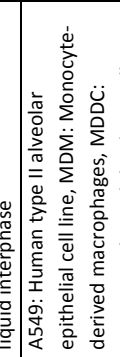 & 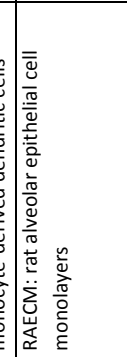 & 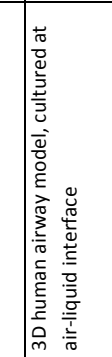 \\
\hline
\end{tabular}


General remarks on currently used in vitro models to study translocation of nanoparticles Currently used in vitro models to study translocation of NPs are mostly based on cell culturing on Transwell $^{\oplus}$ inserts or on the use of ex vivo tissues. Transwell ${ }^{\circ}$ inserts can differ in the type of plastic they are made of and in the pore size of the permeable membrane. These differences between the transwell inserts influence the translocation rate (Cartwright et al., 2012, Geys et al., 2006). Transwell ${ }^{\circ}$ inserts with a larger pore size give increased translocation rates compared to inserts with smaller pore sizes. In addition, some types of plastic of the Transwell ${ }^{\circ}$ inserts yield decreased translocation due to nanoparticle adsorption on the material surface. Therefore, for each single type of nanoparticles, the translocation across the different types of inserts should be tested without cells to assess whether the particles are not withheld by the insert itself.

Next to this, different cell types are used, which clearly influences the translocation and the mechanism of translocation. When cell types lack the formation of tight junctions, NPs can easily pass between the cells resulting in a higher translocation rate compared to cell types that do form tight junctions. To prevent paracellular transport, epithelial cells forming tight junctions should be preferred. Also, the addition of surfactant or surfactant-producing cells can influence the translocation. When NPs deposit in the lungs, they first come in contact with the pulmonary surfactant. This layer might be a very important barrier for NPS because of electrostatic repulsion (for negatively charged NPs) and mucus entrapment (for positively charged NPS) as has been observed after oral exposure (Lai et al., 2007, Norris et al., I998, Szentkuti and Lorenz, I 995, Hussain et al., 200 I). Lastly, the use of specific cells types in co-culture on top of an epithelial layer can alter the translocation rate of the NPS. For instance, alveolar macrophages in lung models can decrease the translocation rate by uptake of NPs. The addition of these specific cell types can improve the in vitro models by more closely mimicking the in vivo situation in which for example the lungs are protected from particles via phagocytosis by alveolar macrophages.

Some reported in vitro models use primary cells, while others use immortalized cell lines. On the one hand, primary cells have a more differentiated phenotype compared to cell lines. On the other hand, the isolation of primary cells is often experimentally challenging: the cells dedifferentiate after isolation, differ from batch to batch and proliferate very slow. Cell lines are easy to work with, well characterized and more homogenous, but they show only few characteristics of differentiated cells. Overall they only poorly represent the in vivo situation (Hartung et al., 2002, Klein et al., 20 I I). In in vitro translocation studies, primary rat alveolar cells have also been frequently used (Fazlollahi et al., 20 I I, Geys et al., 2009, Yacobi et al., 2008). Ex vivo models maintain the complexity of the physiological barriers (Saunders, 2009). However, transport studies in ex vivo models are technically challenging, time consuming and can require large amounts of the test substance.

Advances in the field of miniaturized and microfluidic devices have recently led to the concept of organ-on-a-chip models (Bhatia and Ingber, 20 I 4, Huh et al., 20 I 2, Moraes 
et al., 20I 2, van der Meer and van den Berg, 20I2), which can be seen as hybrid devices combining cells and micro-fabricated structures aiming to recapitulate the dynamic physical, cellular and functional features of human tissues (Huh et al., 20 Io, Ardavin et al., 200 I, Huh et al., 2013, Schimek et al., 2013, Wagner et al., 2013). These devices first provide a high control on the cell microenvironment (e.g., physical and chemical parameters) together with dynamic culture conditions, since they are embedded in a microfluidic format (Whitesides, 2006). Furthermore, these devices can include active elements, which enable to expose cells to mechanical stimuli and surface strains, by stretching the substrate on which they are grown (Sinha et al., 2015). These approaches mostly rely on 3D cell culture conditions, which are acknowledged to better mimic the in vivo conditions compared to conventional monolayer models (Harink et al., 2013).

Over the years, examples of organ-on-a-chip models in the literature have diversified, and for instance include gut (Kim and Ingber, 20 I3), lung (Huh et al., 20 IO, Nalayanda et al., 2010, Huh et al., 2007), or even blood-brain barriers (Huh et al., 2013, Wolff et al., 2015, Griep et al., 2013). These models can be of particular interest for the field of nanotoxicology. Huh et al., for instance, have developed a complex lung-on-a-chip model, which recapitulates movements associated with breathing that is accompanied by cyclic stretching of cells (Huh et al., 20I0). This device has been applied for nanoparticle testing, showing that breathing motion is essential for such nanotoxicological assays. Finally, organ-on-a-chip devices are amenable to parallelization and automation; they are easily coupled to virtually any detection technique, and different organ models can even be combined on one device (Wagner et al., 2013) to eventually mimic the whole body/organism. The availability of such complex and in vivo-like models is expected to facilitate the implementation of the 3 R's legislation regarding animal experimentation (Marx et al., 20I2, van de Stolpe and den Toonder, 2013).

\section{Accuracy of current in vitro translocation models compared to in vivo data}

In vitro translocation studies show mostly limited transport of NPs across the lungs. In the sections below, we discuss the comparison between in vitro data and available in vivo data. We included in vivo studies that measured the bioavailability of similar NPs compared to their in vitro counterparts. However, most in vivo data come from separate studies in which not exactly the same type of nanoparticle is used: for instance, the NPs differ in size. Therefore, we first compared data from studies using NPs of the same chemical composition although they sometimes differed for other characteristics. In table 2, we included studies that used exactly the same NPs in both an in vivo and an in vitro design.

The translocation rate between in vitro lung barrier models and in vivo inhalation studies differs depending on the tested NPs (table 2). In addition, some studies determined the cellular uptake and not the translocation rate of NPs. The information on their localization can also be used in the comparison between in vitro and in vivo data. 
For the most frequently used polystyrene NPS, the translocation rate in both in vitro and in vivo is very low at I day after exposure. Depending on the pore size of the inserts, the translocation is $0-6 \%$ in vitro against $0.05-2 \%$ in vivo. However, one in vivo study shows accumulation over time of polystyrene NPs in the thymus (Sarlo et al., 2009), which cannot be demonstrated in an in vitro lung model. One in vitro study showed a size-dependent translocation rate (Yacobi et al., 2008), which is in line with the size-dependent distribution shown after oropharyngeal aspiration (Sarlo et al., 2009). Also for gold NPs, the in vitro translocation rate that ranges from $0.5 \%$ to $5.2 \%$ (Brandenberger et al., 20Iob) is comparable to the in vivo translocation to the liver of $0.7 \%$ to $2.8 \%$ (Sadauskas et al., 2009, Sung et al., 20 I ra, Takenaka et al., 2006, Yu et al., 2007). For cerium oxide, quantum dots, silica, and titanium dioxide NPs, different translocation rates are found in vitro and in vivo. For cerium oxide, the translocation rate in vivo is very low, and only about $10 \%$ of the inhaled cerium oxide was detected in the lungs (Geraets et al., 20 I2), while $80 \%$ of the particles was internalized in cells in vitro (Raemy et al., 20I I). After inhalation exposure, quantum dots could be detected in the liver and kidney at relative high rates of $15 \%$ and $5 \%$, respectively (Ma-Hock et al., 2012). However, in vitro, no translocation of quantum dots across primary rat alveolar epithelial cells was observed (Fazlollahi et al., 20 I I, Geys et al., 2009). Silica NPs were able to translocate across monolayers of CALU-3, NCI-H292 and A549 cells in vitro (George et al., 2015), but were not detected in tracheobronchial lymph nodes after inhalation exposure (Arts et al., 2007). However, the silica content was not measured in any other tissue or organ besides the lung and lymph nodes, so there might have been some in vivo translocation to the blood, liver and other organs. Finally, titanium dioxide NPs were internalized by A549 epithelial cells, monocyte-derived macrophages, and monocyte-derived dendritic cells in vitro (Muller et al., 2010, Rothen-Rutishauser et al., 2008), while the in vivo studies showed that most NPs were remained in the alveolar space in the lung lining fluid directly after exposure, and were found later on mostly inside alveolar macrophages (Creutzenberg et al., 20 I 2, Geiser et al., 2005, Oberdorster et al., 1994).

The above-mentioned studies did not test exactly the same NPs for the in vitro and in vivo setups. Arai et al. (2014) did test the same silver NPs in vitro and in vivo. However, they did not measure the in vitro NP translocation but the presence of silver material in cells. They concluded that, in vivo, silver ions appear to translocate from the lungs to other tissues rapidly. In vitro, $\mathrm{Ag}$ from $\mathrm{AgNO}_{3}$ binds to metallothioneins, whereas agNPs accumulate in lysosomes (Arai et al., 2014). Multi-walled carbon nanotubes (MwCNTs) were jointly tested in vitro and in vivo. However, this study focused on the development of pulmonary fibrosis and did not measure tissue distribution or translocation. The results did show however that MWCNTs are taken up by alveolar macrophages both in vivo and in vitro (Taylor et al., 2014). 


\section{Analytical techniques to quantify and characterize nanoparticle translocation in vitro and in vivo}

Meaningful interpretation and comparison of the results obtained using different in vitro experiments and extrapolation to in vivo data requires reliable characterization of the NPs and their aggregates, as well as matrix-based influences on the NPs. Therefore, appropriate analytical techniques should be applied to determine the nanoparticle sizedistribution, composition and concentration in the experimental samples.

Widely used methods to detect NPs in liquid dispersions are dynamic light scattering (DLS), centrifugal liquid sedimentation (CLS) (Braun et al., 20 I I, Murdock et al., 2008, Nickel et al., 20I4, Powers et al., 2006, Cascio et al., 2015) and nanoparticle tracking analysis (NTA) (Filipe et al., 20I0, Vasco et al., 20 Io). These methods allow determining an average size or size distribution-related to the measured intensity signal. NTA is able to count and size NPs in aqueous media at $\mu \mathrm{g} / \mathrm{L}$ to $\mathrm{mg} / \mathrm{L}$ concentrations (Filipe et al., 20 Io, Vasco et al., 20IO). Both DLS and NTA are highly dependent on the material properties of the particles since the scattered light of the individual particles must be sufficiently strong for detection. CLS is more robust since particles are size-separated before their actual detection and sizing. Transmission and scanning electron microscopy (TEM, SEM) are other alternative techniques to visualize NPs (Zhang et al., 20 I 2, Dudkiewicz et al., 20 I I). If pure nanoparticle dispersions are analysed, EM is currently the only technique that reliably covers the entire size range down to I $\mathrm{nm}$. In cells or tissues, the minimal particle size that can be detected is around $20 \mathrm{~nm}$, depending on the electron density of the NPs (De Jong et al., 20I0). Furthermore, EM distinguishes size aggregates and primary particles. Other imaging techniques are atomic force microscopy (AFM)(Brown et al., 20I3) and particle-Induced X-ray Emission (PIXE) spectroscopy (Lozano et al., 20 I 2, Lozano et al., 2013).

Elemental information about the sample can also be obtained by atomic spectrometry methods like inductively coupled plasma optical emission spectrometry (ICP-OES) (Elzey et al., 20I2) and ICP mass spectrometry (ICP-Ms) (Krystek, 20 I2, Krystek et al., 20I3), especially in single particle mode (sp ICP-Ms) (Laborda et al., 20I4, Pace et al., 20I I, Peters et al., 20 I 4a). The smallest particle sizes that can be determined are around $20 \mathrm{~nm}$ for silver and gold NPs. For $\mathrm{TiO}_{2}$ and $\mathrm{SiO}_{2}$, NPs size detection limits are around 50 and $200 \mathrm{~nm}$; however, recent experiments suggest that the size detection limit may become lower in the next few years. Another promising possibility is to combine size-specific techniques, that separate particles from each other, like hydrodynamic chromatography (HDC) or field-flow-fractionation ( $\mathrm{FFF}$ ) with atomic spectroscopy techniques, that characterize particles, like ICP-MS (Hassellov et al., 2008, Peters et al., 20 I4b, Striegel and Brewer, 20I2, Von der Kammer et al., 20II), (Bednar et al., 2013). Currently, asymmetric flow-field flow fractionation (AF4) is the most successfully used variant of FFF (Zattoni et al., 20I4). In in vitro testing, AF4 coupled to ICP-Ms becomes a powerful tool to investigate for example time dependent uptake of medium-sized silver NPS 
(Krystek et al., 2015). Laser ablation (LA)-ICP-MS has been used to quantify gold NPs in single cells (Wang et al., 2014a).

Most of the detection methods described above generally require sample preparation procedures. However, only little information concerning sample preparation techniques is available in the literature (Szakal et al., 20I4). Aqueous media containing nanoparticles only need limited sample preparation; samples may be sonicated or tip-sonicated to suspend materials and proteins like bovine serum albumin (BSA), or detergents like sodium dodecylsulfate (SDS) may be added to stabilize nanoparticle suspensions (Jensen et al., 20II). For other matrices, matrix removal or nanoparticle isolation from the matrix can be achieved by physical processes such as centrifugation, filtration, column techniques or cloud point extraction, or by chemical or enzymatic destruction of the matrix (Loeschner et al., 20I4, Peters et al., 20 I4a, Peters et al., 20 I4b).

Overall, different techniques are available to determine the nanoparticle size-distribution, composition and concentration in experimental samples. Attention should be paid to the limits of the chosen techniques and to adequate sample preparation.

\section{CONCLUSIONS ON COMPARISON OF IN VITRO TRANSLOCATION MODELS WITH IN VIVO DATA}

While comparing the in vitro translocation to in vivo data, we noticed that very few studies tested exactly the same nanoparticles in in vitro and in in vivo settings. It seems that most studies are performed either in vitro or in vivo. Therefore, we focused our comparison on the nanoparticle chemical composition although the NPs studied sometimes differ in other particle characteristics like size. Interestingly, this approach revealed that for some NPs, the in vitro translocation is similar to the in vivo translocation. An example is the translocation of polystyrene and gold NPs in in vitro lung barrier models. However, for other NPs, major differences appear to exist between the in vitro translocation rate and the in vivo translocation.

These differences in translocation may be caused by the many differences existing between the in vitro barrier models and the in vivo study designs. First of all, the in vitro models mostly use an acute exposure of maximum 24 hours and a short postexposure time, while for example in vivo inhalation studies vary from I hour to I 3 weeks inhalation exposure, 5 days a week, 6 hours per day with post-exposure periods of up to I year. Long-term exposures are not conceivable in an in vitro setup, although this would be a more realistic exposure scenario. Second, exposure concentrations between the in vitro and in vivo studies differ. In in vitro experiments, high particle concentrations are sometimes used to be able to detect the NPs in the basolateral compartment, especially when fluorescent NPs are used. These high concentrations might increase the agglomeration state of the NPS and damage the epithelial barriers resulting in a different 
translocation mechanism compared to the in vivo situation. Third, in vitro models have a single basolateral compartment, while in vivo, NPs can translocate to various tissues and organs. In the in vitro setup, saturation might occur while under in vivo conditions NPS can continue translocating, since they are taken up in organs and removed from the blood. This distribution of NPs to various tissues can hamper translocation studies in vivo. Fourth, in vitro models are all static, while in vivo exposure is dynamic. Fifth, NPS adsorb proteins and/or phospholipids in biological fluids such as serum or lunglining fluid (Landsiedel et al., 20 I4b). These proteins or phospholipids form a corona around the particles that affects their uptake and bioavailability (Lesniak et al., 20I2). The proteins that are encountered in in vitro models, for example in foetal calf serum, are completely different from the proteins in vivo. Sixth, many in vitro lung models are submerged, which might alter the nanoparticle characteristics and thus the translocation rate, while most in vivo studies rely on inhalation exposure. 
Table 2. Comparison in vitro and in vivo translocation of nanoparticles

The table shows studies in which similar nanoparticles are tested both in vivo and in vitro.

\begin{tabular}{|c|c|c|}
\hline & & 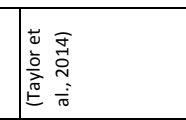 \\
\hline & 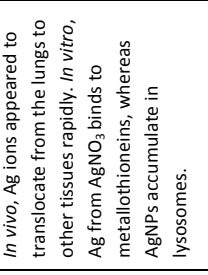 & 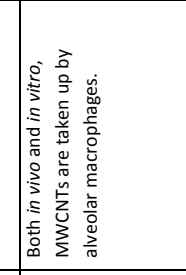 \\
\hline & 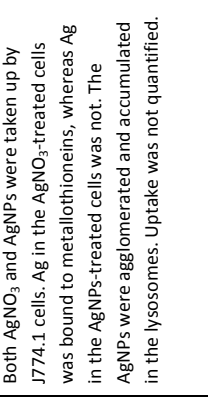 & 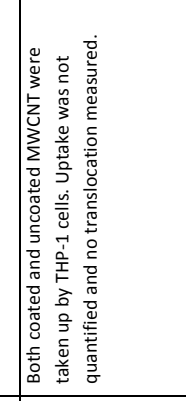 \\
\hline & 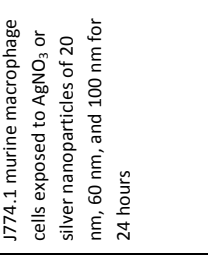 & 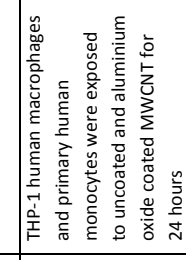 \\
\hline & 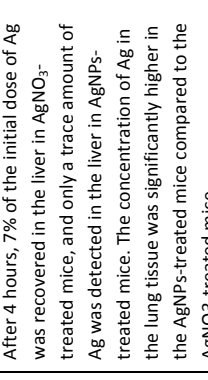 & 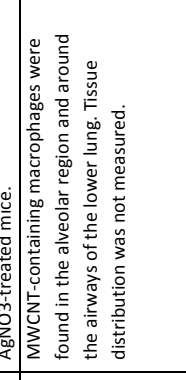 \\
\hline & 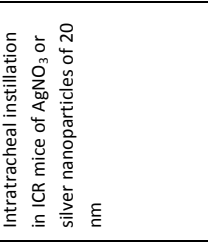 & 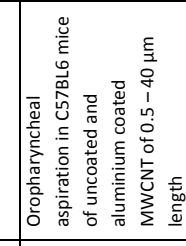 \\
\hline 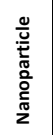 & & 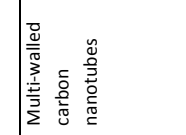 \\
\hline
\end{tabular}




\section{CONCLUSIONS}

The aim of this review was to evaluate the performance of in vitro models that mimic the lung barrier of the human body by comparing -when possible- the in vitro translocation of NPs to their in vivo translocation. A great variety of in vitro models are available to evaluate the translocation of NPs, ranging in complexity from single cell type monolayer to multi-cell (3D) models. Many studies that use in vitro models on inserts focus on the toxicity of NPs and do not include their translocation, while these models might also be suitable for this purpose. In recent years, the availability of analytical detection methods to quantify and characterize the NPs in in vitro settings improved considerably, which provide high quality data that are valuable in studying the relationship between physiochemical properties of the NPs and their translocation. The improved analytical chemicals detection methods also contributed to an increase in in vivo uptake data (bioavailability) of NPS.

Here, while comparing the in vitro translocation to in vivo data, we noticed that very few studies tested exactly the same materials in both settings. Comparing data obtained using NPS of the same chemical composition; we found that for some NPs, the in vitro translocation is similar to the in vivo bioavailability. Examples are the translocation of polystyrene and gold NPS that are in line with the in vivo data. However, for other NPS, major differences were found between the in vitro and the in vivo translocation rate. As discussed in the sections above, many differences exist in the experimental setup between in vitro and in vivo study design that probably account for the poor correlation between these two types of studies. Especially, the changes in the physicochemical characteristics of the NPS caused by presence of lung lining fluid, serum proteins and lipoproteins that form a corona should be taken into account, as these dramatically alter their recognition, uptake and translocation (Lesniak et al., 20I2, Treuel et al., 20I3).

Risk assessment of NPS (as is true for chemicals in general) still heavily relies on in vivo studies. However, the latter must be reduced as far as possible, for numerous reasons. Therefore, there is an urgent need to validate in vitro models using data from animal models, although these animal models do not fully simulate the physiology of humans. How can we establish an in vitro barrier model that has value for the risk assessment of NPs for humans? Ideally, in vitro models reflect the key mechanisms of corresponding in vivo end points, which is not always accomplishable and maybe not required if in vitro models reliably detect NPs that are of concern in vivo (Landsiedel et al., 20I4b). The predictive value of in vitro models can be better assessed by testing exactly the same NPs in the in vitro and in vivo assays. If the ranking of the tested NPs from the lowest translocation rate to the highest translocation rate is the same in both cases, the in vitro models provide information on the internal exposure, which is critical for the ultimate systemic adverse effects. Then, the in vitro models can be considered as suitable for the risk assessment of NPS and will in addition help to reduce animal testing by setting priorities for subsequent in vivo testing. In vitro models with a high predictive value 
do not necessarily have to be complex, but can consist of a single cell type, as long as they give a similar ranking of NPS as obtained in the in vivo situation. However, the mechanism in these models might be completely different from the in vivo situation. To obtain more insight into the mechanisms behind nanoparticle uptake and translocation, in vitro models should be further developed to become physiologically very close to the human body. Such in vitro models must be more complex because the human lung barrier consist of multiple cells types, which are exposed to a low concentration of NPs. A physiologically realistic model will increase the confidence in the NP testing outcome, but it will also be elaborate and expensive to develop and maintain.

When in vitro models are available that have either a high predictive value or are physiologically similar to the human situation, experimental data can be used to develop in silico models that will eventually be able to predict the in vivo bioavailability of NPS from their in vitro translocation rate.

We conclude that the current in vitro models to study the translocation of NPs do not (yet) correlate to the reported in vivo translocation because of many differences between the in vitro and in vivo study designs. However, the use of in vitro models is very promising since they are currently further improved to mimic the in vivo situation more closely by, for example, using co-cultures of different cell types and implementing them in a microfluidic format. When these models are further validated by testing exactly the same NPs in an in vivo setup as in the in vitro model, then, they can be used to determine the internal exposure (bioavailability) of NPS and to set priorities for NPs testing. This will help to reduce the number of animal studies as the risk assessment of nanoparticles, which is now mostly based on in vivo data, can use the data of these validated in vitro barrier models.

\section{ACKNOWLEDGEMENT}

This work is supported by NanoNextNL, a micro- and nanotechnology consortium of the Government of The Netherlands and I 30 partners. 
Hedwig M. Braakhuis ${ }^{1,2,}{ }^{,}$, Marleen Rodenburg ${ }^{3}$, Wim H. de Jong ${ }^{2}$, Margriet V. D. Z. Park ${ }^{2}$

${ }^{I}$ Department of Toxicogenomics, Maastricht University, Po Box 6I6, 6200 MD Maastricht, the Netherlands

${ }^{2}$ National Institute for Public Health and the Environment (RIVM), Po Box I, 3720 BA Bilthoven, the Netherlands

${ }^{3}$ University of Amsterdam, po Box 19568, Io 22 wx Amsterdam, the Netherlands 


\section{Critical evaluation of different insert membranes to study translocation of nanoparticles}




\section{ABSTRACT}

There are concerns that nanoparticles become systemically available after human exposure by crossing biological barriers. Different in vitro models can be used to determine the translocation of nanoparticles. Here, we evaluate the applicability of four different insert membranes for in vitro translocation testing using fluorescent polystyrene NPS with a neutral, positive or negative surface charge. Translocation of NPS was tested in four different types of Transwell ${ }^{\bullet}$ insert membranes; polyester membranes with $0.4 \mu \mathrm{m}$ or 3.0 $\mu \mathrm{m}$ pores, and polycarbonate membranes with $0.4 \mu \mathrm{m}$ or $3.0 \mu \mathrm{m}$ pores. Without cells, the translocation was the highest for the different polystyrene NPs in the polycarbonate insert membranes with a pore size of $3.0 \mu \mathrm{m}$. We chose this type of insert membranes to culture a lung model consisting of epithelial cells and macrophages at the apical side and endothelial cells on the basolateral side. The positively charged polystyrene NPS showed higher translocation and cytotoxicity towards the lung model compared to the neutral and negatively charged ones. For many of the tested NPs concentrations, the translocation was below the fluorescence signal detection limit of $4 \mu \mathrm{g} / \mathrm{ml}$ for the neutral and negatively charged polystyrene NPs and $\mathrm{I} 6 \mu \mathrm{g} / \mathrm{ml}$ for the positively charged ones. In conclusion, the use of different membrane materials and pore sizes influences the quantity of translocated polystyrene NPs. Overall, based on the results with fluorescent NPs and standard fluorometric equipment, the level of translocation needs to be very high in order to detect the fluorescence signal. The determination of realistic translocation levels of NPs in vitro requires the use of highly sensitive techniques and possibly the use of alternative membrane inserts.

\section{INTRODUCTION}

Nanoparticles (NPs) have attractive and novel properties compared to their bulk counterparts and are therefore used in an increasing number of consumer products (Nanotechnologies, 2014, Braakhuis et al., 2015c). However, there are concerns that these NPs might introduce health risks upon occupational and consumer exposure, especially after inhalation exposure (Maynard and Kuempel, 2005, Christensen et al., 20 Io, Braakhuis et al., 20I4a). Apart from potential adverse effects in the lung, concerns arise that due to their small size, NPs could cross some of the biological barriers of the human body, resulting in systemic exposure to NPS, potentially leading to adverse systemic effects (Braakhuis et al., 2015c). To reduce animal testing, several in vitro models have been developed to test the translocation of NPs. We previously evaluated the existence and performance of in vitro models that mimic the barriers of the human body (Braakhuis et al., 20I 5c). Most in vitro barrier models culture cells on Transwell ${ }^{\circ}$ inserts that can be used to quantify the percentage of NPs that cross the insert as a measure of translocation (Chowdhury et al., 2010, Hermanns et al., 2004, Hermanns et al., 20 Iо, Klein et al., 20 I I, Papritz et al., 20 I0, Rothen-Rutishauser et al., 2008, RothenRutishauser et al., 2005, Geys et al., 2006, George et al., 2015). Transwell ${ }^{\oplus}$ inserts can 
be made of different types of plastics and are manufactured with different pore sizes of the permeable membrane. These differences between the Transwell ${ }^{\circ}$ inserts influence the translocation rate (Cartwright et al., 20 I2, Geys et al., 2006). Transwell ${ }^{\circ}$ inserts with a larger pore size result in higher translocation rates compared to inserts with smaller pore sizes (Geys et al., 2006). In contrast, some types of plastics give a lower translocation rate due to nanoparticle adsorption on the material surface (Braakhuis et al., 2015c, Cartwright et al., 20I2). In addition, differences in inserts membranes influence the growth and barrier integrity of the cells cultured on it. Most reported in vitro models do not explain their choice for a specific insert type although this might influence the cell growth and nanoparticle translocation. Different insert membranes that vary in both the pore size and the type of plastic have never been compared in detail to evaluate their applicability for NPs translocation testing. In this study, we evaluate the applicability of four different insert membranes to grow cells with good barrier integrity and for in vitro translocation testing using fluorescent polystyrene NPs with different surface charges.

\section{MATERIALS AND METHODS}

\section{Nanomaterials}

Fluorescent polystyrene nanoparticles of an average nominal diameter of $50 \mathrm{~nm}$ were purchased from Magsphere (Pasadena, USA). The NPs had a red fluorophore core (Ex/ Em: 544/630) and a neutral, aminated or carboxylated surface modification giving them a neutral, positive or negative surface charge, respectively. The NPS were supplied in the form of stock dispersions of $26.25 \mathrm{mg} / \mathrm{ml}$ in Milli-Q ultrapure water (MQ). All particles were negative for endotoxin contamination in the limulus amebocyte lysate (LAL) test.

\section{Nanomaterial characterization}

Nanoparticle size distribution and surface charge were determined in MQ and in complete tissue culture medium of DMEM/FI 2 and RPMI in ratio IO:I (for details see below) without foetal calf serum (FCS) in a concentration of I mg/ml after 24 hours incubation using the dynamic light scattering (DLS) technique. The DLs measurements were performed using a Malvern Zeta Sizer (Worchestershire, uK). The hydrodynamic diameter was calculated based on the average of ro runs of the intensity weighted $\mathrm{Z}$-average performed at $25^{\circ} \mathrm{C}$ using the cumulative fit function performed in triplicate.

\section{Insert membranes}

Four different types of Transwell $^{\circ}$ insert membranes were used; polyester membranes with $0.4 \mu \mathrm{m}$ pores, polycarbonate membranes with $0.4 \mu \mathrm{m}$ pores, polyester membranes with $3.0 \mu \mathrm{m}$ pores and polycarbonate membranes with $3.0 \mu \mathrm{m}$ pores (Costar, Corning Inc., NY, USA). 


\section{Cells and cell culture conditions}

Human bronchial epithelial cells I6HBEI 40-cells (I6HBE) were kindly provided by Dr. Gruenert (University of California, San Francisco, USA) and cultured in DMEM/FI2 medium supplemented with $5 \%$ FCS, IOO $\mathrm{U} / \mathrm{ml}$ penicillin, Ioo $\mu \mathrm{g} / \mathrm{ml}$ streptomycin, 2 $\mathrm{mM} \mathrm{L}$-glutamine and $2.5 \mu \mathrm{g} / \mathrm{ml}$ fungizone (further referred to as complete DMEM/FI2 medium).

Human umbilical vein endothelial cells (HUVEC) were purchased from Life Technologies (Thermo Fisher Scientific Inc., the Netherlands) and cultured in Mzoo supplemented with Io $\mathrm{ml}$ low serum growth supplement (LSGS), Ioо U/ml penicillin, Io० $\mu \mathrm{g} / \mathrm{ml}$ streptomycin and $0.5 \mu \mathrm{g} / \mathrm{ml}$ fungizone (further referred to as complete M200 medium). Human acute monocytic leukemia cells (THP-I) were purchased from American Type Culture Collection (ATCC, Rockville, USA) and cultured in RPMI medium supplemented with IO\% FCS, I00 $\mathrm{U} / \mathrm{ml}$ penicillin, and $100 \mu \mathrm{g} / \mathrm{ml}$ streptomycin (further referred to as complete RPMI medium). To differentiate the THP-I monocytes into macrophages, the cells were seeded in a $75 \mathrm{~cm}^{2}$ culture flask in a density of 500.000 cells $/ \mathrm{ml}$ in the presence of $30 \mathrm{ng} / \mathrm{ml}$ PMA for 5 subsequent days. At day 5 , the medium was removed and replaced by full culture medium for 3 days.

The cells were incubated at $37{ }^{\circ} \mathrm{C}$ in a $100 \%$ humidified atmosphere containing $5 \%$ $\mathrm{CO}_{2}$. When cells reached confluency, the epithelial and endothelial cells were released enzymatically (0.05\% trypsin-eDta for I6HBE and HUVEC, Accutase for THP-I) and transferred to a new culture flask of $75 \mathrm{~cm}^{2}$. The medium was renewed every 2-3 days.

\section{Preparation of the lung model}

A lung model (modified from the model recently published by Luyts et al. (2015) (Luyts et al., 2015)) was prepared on non-coated polycarbonate Transwell ${ }^{\circ}$ insert membranes with $3.0 \mu \mathrm{m}$ pores and a surface area of $0.33 \mathrm{~cm}^{2}$ (inserts fit in a 24-wells plate). HUVEC cells were seeded in a density of 50.000 cells $/ \mathrm{cm}^{2}$ on the basolateral side of the transwell inserts. After 2 hours, the transwell inserts were returned to their original orientation and $16 \mathrm{HBE}$ cells were seeded in a density of 100.000 cells $/ \mathrm{cm}^{2}$ on the apical side. After 7 days, THP-I macrophages were added to the bi-culture model at the apical side in a density of $50.000 \mathrm{cell} / \mathrm{cm}^{2}$ (I THP-I cell per IO I6HBE cells). A schematic representation of the lung model is shown in figure $\mathrm{I}$.

\section{Measurements of transepithelial electrical resistance (TEER)}

For measurements of the barrier integrity, the lung model was prepared on all four different insert membranes. The transepithelial electrical resistance (TEER) was measured using the Evom2 Voltohmmeter supplemented with an EndOhm-6 chamber (World Precision Instruments, Inc., FL, USA). The TEER values were corrected for the resistance of a cell-free transwell insert and for the surface area of the transwell insert. 
A

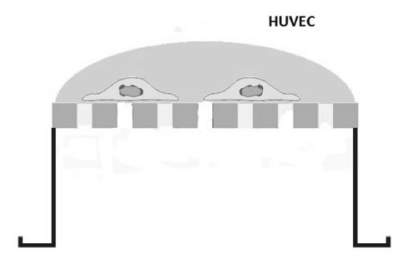

B

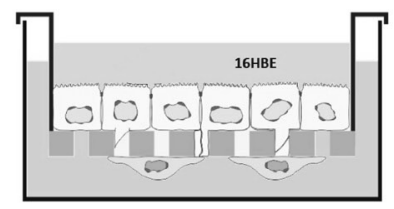

C

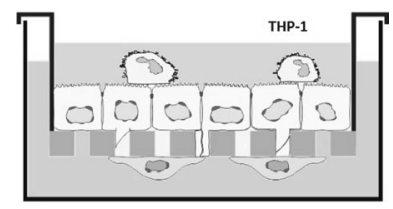

Figure 1. Schematic representation of the lung barrier model. HuvEcs were seeded at the basolateral side of the Transwell ${ }^{\circledR}$ insert membrane (A), after 2-3 hours 16 HBE cells were seeded at the apical side of the insert membrane (B), finally differentiated THP-1 cells were added to the apical side of the bi-culture (C). Figure adapted from Rothen-Rutishauser et al. 2005 (Rothen-Rutishauser et al., 2005).

\section{Metabolic activity}

To determine the potential toxicity of polystyrene NPs towards the lung model, cell metabolic activity was evaluated using the wsT-I cell Proliferation Reagent (Roche, Almere, the Netherlands). The metabolic conversion of wST-I by the cells and the ability of polystyrene NPS to scatter or absorb light or to interfere with the WST-I reagent were assessed in 96-wells plates with a co-culture of I6HBE and THP- I according to methods described earlier. Briefly, cells were exposed to polystyrene NPs for 24 hours and incubated with wST-I reagent for the last 3 hours of the exposure. Absorbance was measured using a SpectraMax I90 scanning multiwell spectrophotometer (Molecular Devices, Sunnyvale, USA) at a wavelength of $440 \mathrm{~nm}$ and a reference wavelength of $620 \mathrm{~nm}$. The absorbance of polystyrene NPs in tissue culture medium, measured in the absence of cells, was subtracted from the total absorbance of the nanoparticle treated cells.

\section{Nanoparticle translocation}

The translocation of fluorescent polystyrene NPS was first determined in the different Transwell ${ }^{\bullet}$ inserts without cells to investigate whether the NPs are not withheld by the insert itself and are able to cross the inserts membranes. NPS were diluted in complete culture medium of DMEM/FI 2 and RPMI in ratio IO:I without FCS and without phenol red $($ IOO $-1000 \mu \mathrm{g} / \mathrm{ml})$. Next, $200 \mu \mathrm{l}$ of the NP suspension was added to the apical side of the Transwell ${ }^{\circ}$ insert and incubated for 24 hours at $37^{\circ} \mathrm{C}$. After incubation, the culture medium from the apical and the basolateral side of the insert membranes was transferred to a dark-sided clear-bottom 96 well plate. Subsequently, the fluorescence signal was measured using a Tristar ${ }^{2}$ Multimode Reader LB 942 (Berthold Technologies) at $544 / 630 \mathrm{Ex} / \mathrm{Em}$ wavelengths.

Next, the lung model consisting of lung epithelial cells, macrophages and endothelial 
cells was prepared as described above on polycarbonate inserts with $3.0 \mu \mathrm{m}$ pores to determine the translocation rate of fluorescent polystyrene NPS $($ I OO $-400 \mu \mathrm{g} / \mathrm{ml})$ with cells. The lung model was not prepared on the other insert types because they showed no detectable translocation without cells.

\section{Statistical analysis}

All experiments were performed in triplicate. The data was analysed by the Kruskal Wallis nonparametric test (Graphpad Prism). Statistical significance is indicated with $\mathrm{a}^{*}(p=<0.05)$. In all graphs, error bars represent the standard deviation of the mean.

\section{RESULTS}

\section{Nanomaterial characterization}

The nanoparticle characteristics are summarized in table $\mathrm{I}$. The neutral, positive and negative charged NPs had an average hydrodynamic size of 50.4, 49.6 and 49.1 nm in MQ and a size of 57.5, 50.2 and $48.3 \mathrm{~nm}$ in culture medium of DMEM/FI 2 and RPMI in ratio IO:I without FCS, respectively. In MQ, the zeta potential of the aminated and carboxylated NPS confirmed their positive and negative surface charge. The neutral NPS had a negative surface charge in MQ. In culture medium without FCS, the differences in zeta potential between the different particles were decreased.

Table 1. Size and Zeta potential of fluorescent polystyrene NPS.

\begin{tabular}{|c|c|c|c|c|}
\hline Nanoparticles & $\begin{array}{c}\text { Average size }(\mathbf{n m}) \\
\text { in MQ }\end{array}$ & $\begin{array}{c}\text { Zeta potential }(\mathbf{m V}) \\
\text { in MQ }\end{array}$ & $\begin{array}{c}\text { Average size } \\
(\mathbf{n m}) \text { in culture } \\
\text { medium w/o FCS }\end{array}$ & $\begin{array}{c}\text { Zeta potential } \\
(\mathbf{m V}) \text { in culture medium } \\
\mathbf{w} / \mathbf{o} \text { FCS }\end{array}$ \\
\hline Neutral & 50.4 & -58.1 & 57.5 & -28.8 \\
Aminated & 49.6 & 41.0 & 50.2 & 22.1 \\
Carboxylated & 49.1 & -67.4 & 48.3 & -28.7 \\
\hline
\end{tabular}

\section{Measurements of transepithelial electrical resistance (TEER)}

In each of the four insert membranes, the TEER was measured to monitor cell layer confluence and barrier integrity of the lung model at day 3, 5, 8, 9 and Io after seeding (figure 2). The TEER values were the highest for the polyester inserts with $0.4 \mu \mathrm{m}$ pores that increased up to an average of $1782 \Omega^{*} \mathrm{~cm}^{2}$ at Io days after seeding. The TEER values decreased when using inserts with larger pores. The lowest TEER values were measured in the polycarbonate inserts with $3.0 \mu \mathrm{m}$ pores that had an average TEER value of I 67 $\Omega^{*} \mathrm{~cm}^{2}$ at Io days after seeding. 


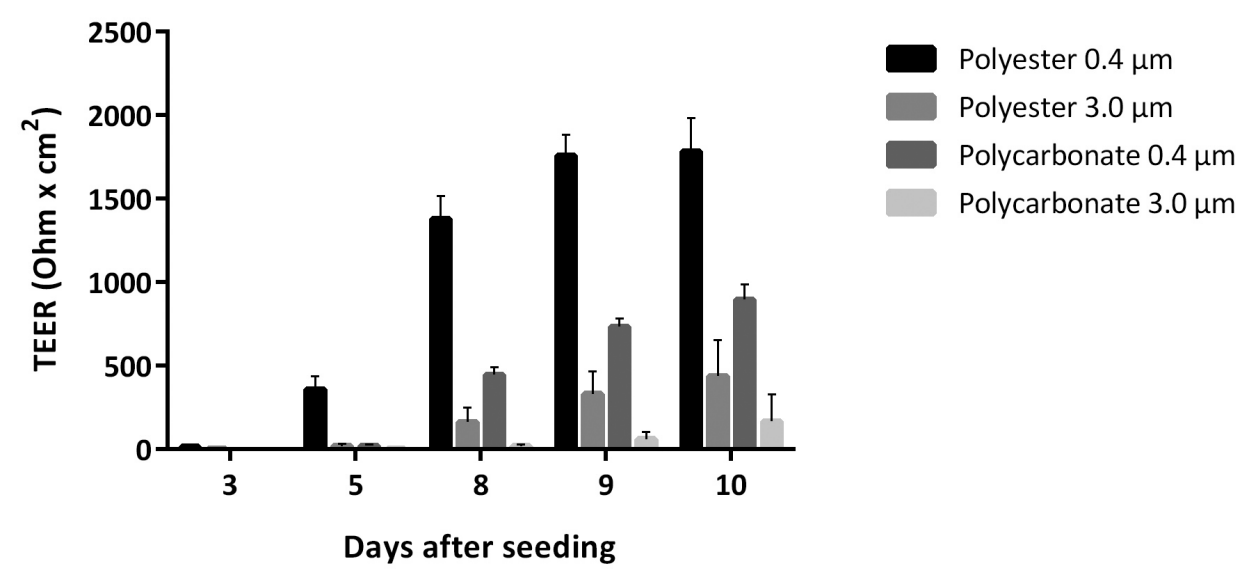

Figure 2. Trans-epithelial electrical resistance (TEER) in a co-culture of endothelial cells, epithelial cells and alveolar macrophages cultured on different types of Transwell inserts $(n=3)$. The TEER values are the highest in the polyester insert membranes with the smaller pore size of $0.4 \mu \mathrm{m}$.
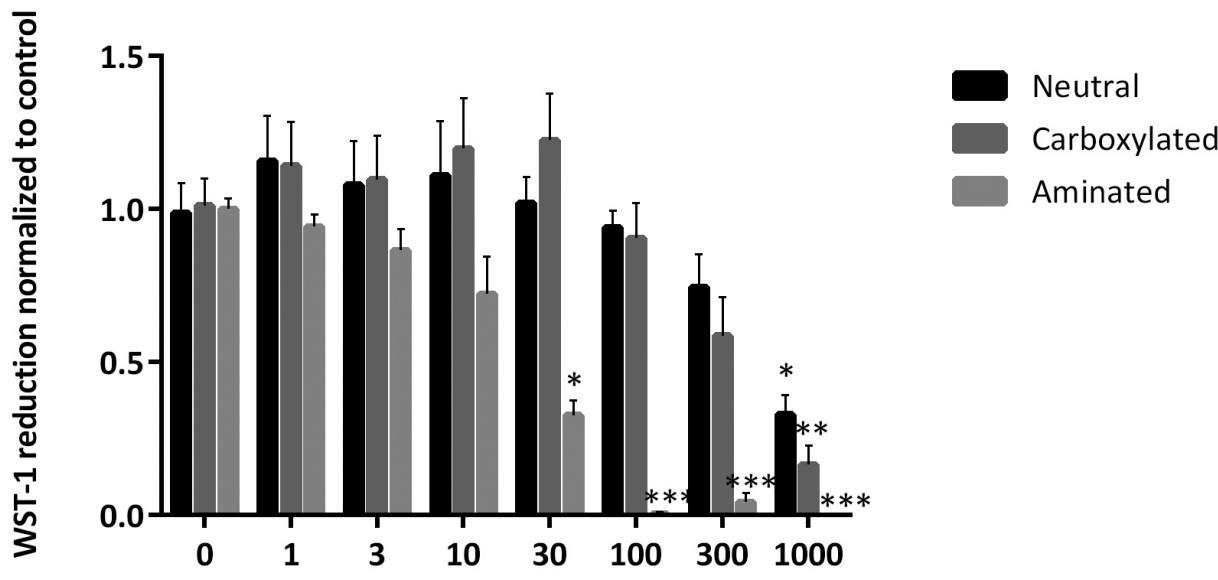

Aminated

\section{Particle concentration $(\mu \mathrm{g} / \mathrm{ml})$}

Figure 3. Metabolic activity of the lung barrier model after 24 hours exposure to polystyrene nanoparticles with different surface charges. Bars represent mean values with their standard deviation $(\mathrm{n}=3$ ). After exposure to $30 \mu \mathrm{g} / \mathrm{ml}$ and above of positively charged polystyrene NPS and after exposure to $1000 \mu \mathrm{g} / \mathrm{ml}$ of neutral and negatively charged polystyrene NPS, the metabolic activity significantly decreased $\left({ }^{*}=p<0.05,{ }^{* *}=p<0.01,{ }^{* * *}=p<0.001\right)$. 


\section{Metabolic activity}

The metabolic activity of the lung barrier model after exposure to differently charged polystyrene NPs is shown in figure 3. Exposure to neutrally and negatively charged fluorescent polystyrene NPs for 24 hours resulted in a significant decrease in metabolic activity at the highest concentration tested of $\mathrm{I} 000 \mu \mathrm{g} / \mathrm{ml}(\mathrm{p}=0.0 \mathrm{I} 2$ and $\mathrm{p}=0.009$, respectively). At the lower concentrations, there was no effect of the neutral and negative polystyrene NPs on cell viability. Exposure to positively charged fluorescent polystyrene NPs for 24 hours resulted in a significant decrease in metabolic activity at concentrations of $30 \mu \mathrm{g} / \mathrm{ml}(\mathrm{p}=0.0 \mathrm{I} 2)$ and above. Positively charged polystyrene NPS cause more cytotoxicity to a co-culture of I6 $\mathrm{HBE}$ and THP-I cells compared to neutral or negatively charged polystyrene NPS.

Table 2. Detection limits of the neutral, positive and negative polystyrene NPS, given as concentration $(\mu \mathrm{g} / \mathrm{ml})$ and as the percentage of the apical concentration (\%) when measured at the basolateral side of the insert membrane.

\begin{tabular}{|c|c|c|c|c|c|c|c|}
\hline $\begin{array}{c}\text { Polystyrene } \\
\text { nanoparticles }\end{array}$ & $\begin{array}{c}\text { Detection } \\
\text { limit }\end{array}$ & \multicolumn{6}{|c|}{$\begin{array}{c}\text { Translocation needed to reach the detection limit (given as \% of } \\
\text { the apical concentration) for measuring fluorescence signal at } \\
\text { basolateral side of insert membrane using different apical } \\
\text { concentrations }(\boldsymbol{\mu g} / \mathbf{m l})\end{array}$} \\
\hline & & $\mathbf{1 0 0}$ & $\mathbf{2 0 0}$ & $\mathbf{4 0 0}$ & $\mathbf{6 0 0}$ & $\mathbf{8 0 0}$ & $\mathbf{1 0 0 0}$ \\
\hline Neutral & $4 \mu \mathrm{g} / \mathrm{ml}$ & $20 \%$ & $10 \%$ & $5 \%$ & $3.3 \%$ & $2.5 \%$ & $2 \%$ \\
\hline Positive (aminated) & $16 \mu \mathrm{g} / \mathrm{ml}$ & $80 \%$ & $40 \%$ & $20 \%$ & $13.3 \%$ & $10 \%$ & $8 \%$ \\
\hline Negative (carboxylated) & $4 \mu \mathrm{g} / \mathrm{ml}$ & $20 \%$ & $10 \%$ & $5 \%$ & $3.3 \%$ & $2.5 \%$ & $2 \%$ \\
\hline
\end{tabular}

\section{Nanoparticle translocation}

First, we determined the detection limit of the differently charged polystyrene NPs by measuring the fluorescence signal of a standard curve with known particle concentrations. Using fluorescence detection, we could detect a signal above the background when at least $4 \mu \mathrm{g}$ per $\mathrm{ml}$ of NPs was present of the neutral and negative polystyrene NPs. At the apical side of the insert membrane, the exposure concentration was applied in a total volume of $200 \mu \mathrm{l}$. At the basolateral side of the membrane, there was a total volume of I $000 \mu \mathrm{l}$ culture medium. When adding concentration ranges of Io० to $\mathrm{I} 000 \mu \mathrm{g} / \mathrm{ml}$ at the apical side in $200 \mu \mathrm{l}$, the absolute amounts per well are 20 to $200 \mu \mathrm{g}$. This means that for the lowest amount tested of $20 \mu \mathrm{g}$ (equals $100 \mu \mathrm{g} / \mathrm{ml}$ ) at least $20 \%$ should translocate to be able to detect any signal. For the positively charged polystyrene NPS, the detection limit was even higher at $\mathrm{I} 6 \mu \mathrm{g} / \mathrm{ml}$. For these NPs, at least $80 \%$ should translocate to be able to detect any signal at the lowest apical concentration of $100 \mu \mathrm{g} /$ $\mathrm{ml}$. Table 2 shows the detection limits of the polystyrene NPs for each of the tested nanoparticle concentrations, and the percentage of translocation of the polystyrene NPS to obtain values equal to the detection limit. 
Second, we determined the translocation amount of fluorescent polystyrene NPs in the different Transwell ${ }^{\circ}$ inserts without cells to investigate whether the NPs can cross the insert membranes or are withheld by the inserts itself (figure 4). In figure 4, we excluded all measurements that were below the detection limit. For all NPs, the translocation in the polyester membranes with $0.4 \mu \mathrm{m}$ pores was below the detection limit for all tested concentrations. Increasing the pore size to $3.0 \mu \mathrm{m}$ increased the translocation percentage above the detection limit for the positively and negatively charged NPs. In addition, using the polycarbonate membranes also increased translocation compared to the polyester membranes. For the positively charged NPS, the translocation was below the detection limit at particle concentrations of $\mathrm{IOO}$ and 200 $\mu \mathrm{g} / \mathrm{ml}$. However, they showed more translocation compared to the neutral and negatively charged NPS at the higher tested particle concentrations of $400 \mu \mathrm{g} / \mathrm{ml}$ and above. The NPS with a neutral surface charge showed translocation above the detection limit when using polycarbonate inserts with $3.0 \mu \mathrm{m}$ pores; in the other insert types the translocation was below the detection limit. The translocation could be the best detected when using membranes of polycarbonate with a pore size of $3.0 \mu \mathrm{m}$.
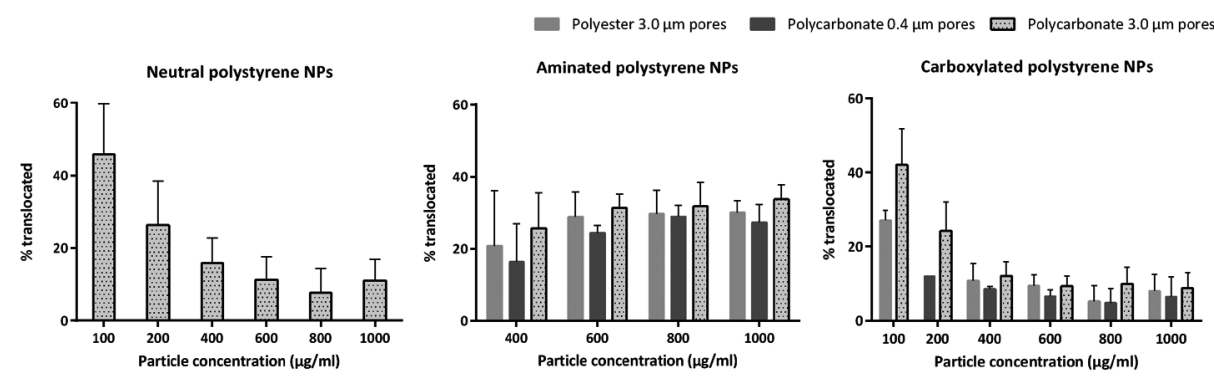

Figure 4. Translocation of neutral (left), positively charged (middle) and negatively charged (right) fluorescent polystyrene NPS in different insert membranes without cells. Bars represent mean values with their standard deviation $(n=3)$. In the polyester membranes with $0.4 \mu \mathrm{m}$ pores, the translocation was below the detection limit for all polystyrene NPS. For the positively charged NPS, the translocation was below the detection limit at particle concentrations below $400 \mu \mathrm{g} / \mathrm{ml}$. The translocation could be the best detected when using membranes of polycarbonate with a pore size of $3.0 \mu \mathrm{m}$.

Finally, the complete lung model was prepared as described above on polycarbonate inserts with $3.0 \mu \mathrm{m}$ pores to determine the translocation rate of fluorescent polystyrene NPs with cells. For these experiments, we chose nanoparticle concentrations of I00, 200 and $400 \mu \mathrm{g} / \mathrm{ml}$ because translocation in polycarbonate membranes with $3.0 \mu \mathrm{m}$ was observed for these concentrations while there was no decrease in metabolic activity for the neutral and negatively charged NPS. TEER values were measured to determine whether the polystyrene NPS affect the barrier integrity (figure 5). Exposure to the neutral and positively charged polystyrene NPs did significantly affect the barrier integrity, while exposure to the negatively charged polystyrene NPs did not. 


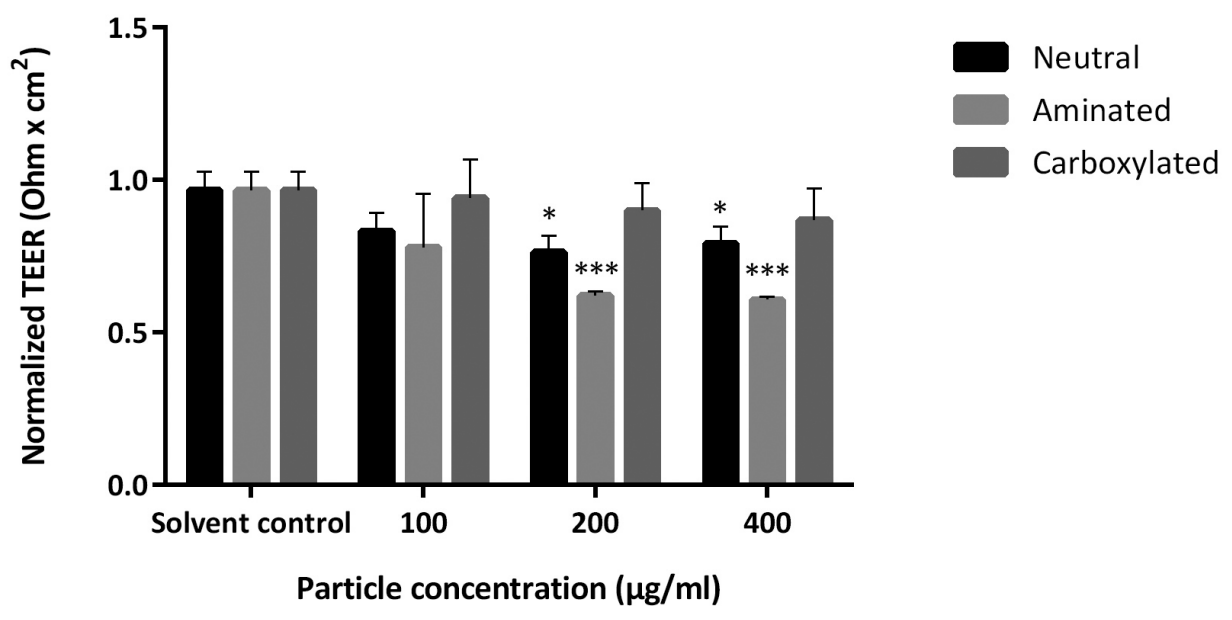

Figure 5. Trans-epithelial electrical resistance (TEER) in a co-culture of endothelial cells, epithelial cells and alveolar macrophages cultured on polycarbonate insert membranes with a pore size of $3.0 \mu \mathrm{m}$ after 24 hours exposure to polystyrene NPS. Bars represent mean values with their standard deviation $(n=3)$. After exposure to neutral and positively charged polystyrene NPS, the TEER is significantly decreased. $\left(^{*}=p<0.05,{ }^{* * *}=p<0.001\right)$.
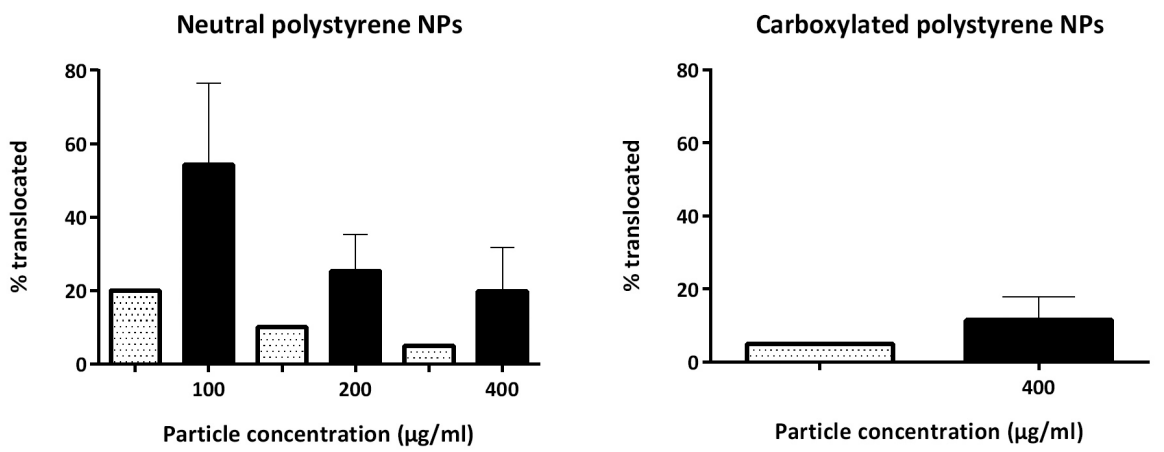

Figure 6. Translocation of neutral (left) and negatively charged (right) fluorescent polystyrene NPS in the lung model cultured on polycarbonate membranes with $3.0 \mu \mathrm{m}$ pores. Bars represent mean values with their standard deviation $(n=2)$. Translocation of positively charged fluorescent polystyrene NPS was below the detection limit. The observed translocation in the lung model is similar to the translocation without cells.

For the positively charged NPS, the translocation in the lung model cultured on polycarbonate inserts with $3.0 \mu \mathrm{m}$ pores was below the detection limit for the tested concentrations of IOO, 200 and $400 \mu \mathrm{g} / \mathrm{ml}$. Translocation across the lung model 
could be detected for the neutral and negatively charged NPs (figure 6). In figure 6, all measurements below the detection limit are excluded. For the negatively charged NPs, translocation was above the detection limit at the highest concentration tested of $400 \mu \mathrm{g} / \mathrm{ml}$. The translocation in the lung barrier model with cells cultured on polycarbonate insert membranes with $3.0 \mu \mathrm{m}$ pores is similar compared to the empty insert membranes when using neutral polystyrene NPs (table 3 ). The positively charged NPs have the highest detection limit and can therefore not be detected in both the insert model with and without cells. However, at $400 \mu \mathrm{g} / \mathrm{ml}$, we measured translocation in the inserts without cells while we did not in the inserts with cells. Similarly, translocation of the negatively charged NPs could be detected in the polycarbonate insert membranes with $3.0 \mu \mathrm{m}$ pores without cells, while we did not detect translocation in the insert membranes with cells (table 3 ).

Table 3. Translocation of differently charged fluorescent polystyrene NPS in polycarbonate insert membranes with $3.0 \mu \mathrm{m}$ pores without cells and with the full lung barrier model consisting of lung epithelial cells, macrophages and endothelial cells. Values represent the mean in \% of the apical concentration with the standard deviation $(n=3)$.

\begin{tabular}{|c|c|c|c|c|}
\hline $\begin{array}{c}\text { Polystyrene } \\
\text { Nanoparticles }\end{array}$ & $\begin{array}{c}\text { Apical } \\
\text { concentration } \\
(\boldsymbol{\mu g} / \mathrm{ml})\end{array}$ & $\begin{array}{c}\text { Translocation } \\
\text { needed to reach } \\
\text { detection limit }\end{array}$ & $\begin{array}{c}\text { Translocation in empty } \\
\text { insert membrane } \\
\text { without cells }\end{array}$ & $\begin{array}{c}\text { Translocation in lung } \\
\text { barrier model with cells }\end{array}$ \\
\cline { 2 - 5 } & 100 & $20 \%$ & $45.8 \%(13.9 \%)$ & $54.3 \%(22.2 \%)$ \\
\cline { 2 - 5 } Neutral & 200 & $10 \%$ & $26.3 \%(12.2 \%)$ & $25.3 \%(9.9 \%)$ \\
\hline \multirow{2}{*}{$\begin{array}{c}\text { Positive } \\
\text { (aminated) }\end{array}$} & 400 & $5 \%$ & $15.8 \%(6.9 \%)$ & $19.8 \%(12 \%)$ \\
\cline { 2 - 5 } & 100 & $80 \%$ & $0 \%$ & $0 \%$ \\
\hline \multirow{2}{*}{$\begin{array}{c}\text { Negative } \\
\text { (carboxylated) }\end{array}$} & 100 & $40 \%$ & $0 \%$ & $0 \%$ \\
\cline { 2 - 5 } & 200 & $20 \%$ & $25.6 \%(9.9 \%)$ & $0 \%$ \\
\cline { 2 - 5 } & 400 & $10 \%$ & $24.2 \%(7.8 \%)$ & $0 \%$ \\
\hline
\end{tabular}

\section{DISCUSSION}

The aim of this study was to evaluate the applicability of the different types of insert membranes for in vitro translocation testing using fluorescent polystyrene NPs. The use of different membrane materials and pore sizes influences the trans-epithelial electrical resistance (TEER) and the translocation of polystyrene NPS. The TEER is the highest when cells are cultured on the $0.4 \mu \mathrm{m}$ polyester insert membranes; indicating optimal barrier integrity can be accomplished using this insert type. The translocation of polystyrene NPs in these inserts without cells is below the detection limit, while the translocation in the other insert membranes is not. When using insert membranes with a larger pore size of $3.0 \mu \mathrm{m}$, the translocation increases but the TEER of the biological barrier prepared on these membranes decreases, indicating that the lung cells are struggling to maintain a 
confluent barrier. Overall, the translocation without cells was the highest for the different polystyrene NPs in the polycarbonate insert membranes with a pore size of $3.0 \mu \mathrm{m}$. This is in line with a previously reported study showing that polystyrene NPs can adhere to polyester membranes and thereby reduce their translocation (Cartwright et al., 20 I2). Another study showed that significant translocation was observed using $3.0 \mu \mathrm{m}$ pores and no translocation was observed using $0.4 \mu \mathrm{m}$ pores (Geys et al., 2006). None of the tested insert membranes seems optimal for testing translocation of NPs as they either provide good barrier integrity but at the same time adsorb NPs, or they adsorb NPs to a lesser extent but provide poor barrier integrity. Still, previous in vitro studies used one type of insert membranes to study translocation of NPs without evaluation of other types of inserts (Chowdhury et al., 20 I o, George et al., 20 I 5, Hermanns et al., 20 I0, RothenRutishauser et al., 2008, Walczak et al., 20I4).

Besides differences in insert membranes, we tested toxicity and translocation of polystyrene NPS with different surface charges. In complete culture medium without FCS, the surface charge of the neutral and carboxylated NPs was both negative, while the surface charge of the aminated NPS was positive. The positively charged polystyrene NPs induced cytotoxicity in the lung model at lower concentrations compared to the other polystyrene NPS. In addition, the positively charged polystyrene NPs showed a higher translocation over insert membranes without cells compared to the neutral and negatively charged polystyrene NPS even though the detection limit of these particles was 4 times higher compared to the other polystyrene NPs. The higher translocation of positively charged polystyrene NPs compared to negatively charged ones was observed before (Yacobi et al., 2008). Generally, positively charged NPs are found to be more cytotoxic in vitro compared to neutral or negatively charged NPs (Nel et al., 2009, Asati et al., 20 Io, Cho et al., 20 2b, Nagy et al., 20 I 2). Regarding the translocation however, in vivo studies have generally found that charged particles attract proteins and thereby reduce their uptake and translocation rate (Choi et al., 20 Io, Choi et al., 2007, Gessner et al., 2002). Because we used complete tissue culture medium without serum in our translocation experiments, there was no formation of a protein corona that could reduce the translocation rate. Probably, the negative charge of the neutral and carboxylated NPS in culture medium caused the NPs to stick to the plastic of the insert membranes while the positive charge of the aminated NPs prevented this.

Fluorescent NPs have the advantage of being easily detectable at low costs. However, the detection limit of the fluorescence signal of $4 \mu \mathrm{g} / \mathrm{ml}$ for the neutral and negative NPS and $\mathrm{I} 6 \mu \mathrm{g} / \mathrm{ml}$ for the positive NPs is very high and the fluorescence signal fades over time (Riehemann et al., 2009). Walczak et al. (2014) did not report similar high detection limits in their study (Walczak et al., 20 I4). In an intestinal model cultured on polyester insert membranes with $3.0 \mu \mathrm{m}$ pores, the translocation of $50 \mathrm{~nm}$ fluorescent polystyrene NPS with a neutral, positive and negative surface charge was $7.8 \%, \mathrm{I} \%$ and ○\%, respectively (Walczak et al., 20 I 4). In the present study, the detection limit (in \%) of the fluorescent polystyrene NPs decreased when using higher apical concentrations. 
This resulted in the detection of positively charged polystyrene NPS in the basolateral compartment after exposure to $400,600,800$ and $1000 \mu \mathrm{g} / \mathrm{ml}$ in both the polycarbonate membranes and in the polyester membranes with the larger pore size. However, these high concentrations affect the viability of the lung model. In vivo, the translocation of negatively charged polystyrene NPs is very low (Mohammad et al., 20I3, Sarlo et al., 2009). After acute aspiration to 44, 276 and $390 \mu \mathrm{g}$ polystyrene NPs of 20 , IO0 and $1000 \mathrm{~nm}$ respectively, most NPs were found in the lungs at I day after exposure (Sarlo et al., 2009). The polystyrene NPs caused mild pulmonary toxicity. All particle sizes were detected in the liver at concentrations below $0.5 \%$. The $20 \mathrm{~nm}$ NPs were detected in the blood, brains and kidneys at concentrations below $0.5 \%$, the other particle sizes were not (Sarlo et al., 2009). In another study, the distribution of 50, I00, 250 , and $900 \mathrm{~nm}$ negatively charged polystyrene NPs was determined after pharyncheal aspiration to a dose of I $575 \mu \mathrm{g}$. Histology of the lungs revealed little inflammation. At 3 hours after exposure, translocation of polystyrene NPs to the liver was minimal with a maximum of I.I\% for the $50 \mathrm{~nm}$ NPs (Mohammad et al., 2013). The highest amount of polystyrene NPs was detected in the lymph nodes at 5 days after exposure; I $2.3 \%$ of the administered dose of the $50 \mathrm{~nm}$ NPS and $6.9 \%, 8 \%$, and $6.2 \%$ of the I00, 250 and $900 \mathrm{~nm}$ NPs, respectively (Mohammad et al., 20I3). Also for other NPs, the reported translocation after inhalation exposure is very low, below $0.5 \%$ of the exposure concentration (Geraets et al., 20 I2, Kreyling et al., 2002, Kreyling et al., 2009, Sadauskas et al., 2009, Braakhuis et al., 20I4b). The comparison between in vivo and in vitro translocation is difficult as for polystyrene NPs no studies are available that tested exactly the same NPs in vivo and in vitro. The results of in vitro studies, provided that they are adequately controlled, can be used to identify relative transfer rates of NPS with different physicochemical characteristics and thus help priority setting for NPs testing; positively charged NPs will probably have a higher systemic bioavailability compared to negative and neutral NPS.

The results of previously performed in vivo studies with polystyrene NPs showed translocation up to I.I\% of a single administered dose, when measured in the first hours after the exposure (Sarlo et al., 2009, Mohammad et al., 2013). In the present study, the detection limit of the red fluorescent NPs is far above this I.I\%, even when applying higher concentrations at the apical side, and therefore the use of fluorescent nanoparticles detection by fluorescence measurement as performed in the present study is not suitable to determine translocation in vitro. Fluorescent NPs of other manufacturers or other colours might improve their detection, but probably more sensitive detection techniques should be used. To detect fluorescent NPs in vitro, conventional fluorescence microscopy or fluorescence correlation spectroscopy can be used (Geys et al., 2006, Ostrowski et al., 2015) that are able to detect aggregates or densely packed NPs. Using fluorescence correlation spectroscopy, translocation of positively and negatively charged polystyrene NPs $(25 \mu \mathrm{g} / \mathrm{ml})$ in polyester insert membranes with $3.0 \mu \mathrm{m}$ pores without cells was $52.7 \%$ and $67.5 \%$, respectively (Geys et al., 2006). These measurements are more reliable compared to the values reported in this study because in our study an 
apical concentration of $25 \mu \mathrm{g} / \mathrm{ml}$ would have to show a translocation of $80 \%$ for the neutral and negatively charged NPs and for the positively charged NPs, the signal would still be below the detection limit even if ı $10 \%$ of the particles translocated. Fluorescence correlation spectroscopy can be optimized using confocal laser scanning microscopy (van Schooneveld et al., 2008). For detection of metal NPS, atomic spectrometry methods like inductively coupled plasma optical emission spectrometry (ICP-OEs) (Elzey et al., 2OI 2) and ICP mass spectrometry (ICP-MS) can be used (Krystek, 20I2, Krystek et al., 20I3). However, they are more expensive compared to fluorescence measurements. Using conventional ICP-MS, the detection limit of most metal NPs is about O.OI $\mu \mathrm{g}$ per gram of tissue and $0.0 \mathrm{I} \mu \mathrm{g} / \mathrm{ml}$ in liquids (Krystek et al., 2013). Another option is to label NPS with an appropriate radioisotope (Gibson et al., 20I I). Radioisotopes of silver, carbon and indium have been used to label NPs to study their distribution in the body (Ostrowski et al., 2015, Al-Sid-Cheikh et al., 2013, Al-Hallak et al., 2012, Lee et al., 2006). The radiation of these isotopes is usually detected and quantified by making autoradiographs (Ostrowski et al., 2015). By adjusting autoradiography for light or electron microscopy, the sensitivity of the technique can be increased (Holzhausen et al., 2013, Groger et al., 2013 , Kennel et al., 2008). A disadvantage of using radioactive materials is that it requires dedicated laboratory facilities, strict safety precautions and it generates radioactive waste.

In conclusion, both the type of insert membrane and the particle characteristics influence the translocation of NPs, indicating the importance of testing the appropriate insert membrane for each different nanoparticle. None of the tested insert membranes seems optimal for testing translocation of NPs; either good barrier integrity is provided but NPs are adsorbed to the membrane, or NPs are adsorbed to a lesser extent but the barrier integrity is flawed. The results of studies using these membranes should therefore be interpreted with caution. A solution might be to develop a thin insert membrane that does not withhold NPS and is suitable to test all types of NPs. Such a model should be validated using in vivo data. Using a fluorescence spectrophotometer to detect fluorescent nanoparticles is not suitable to determine translocation in vitro because of the poor detection limit. To evaluate the translocation of NPs in vitro, more sensitive detection techniques should be used.

\section{ACKNOWLEDGEMENTS}

This work was supported by the project 'Integrated Risk Assessment of Nanomaterials' from the National Institute for Public Health and the Environment and by the NanoNextnl program 'Risk Analysis and Technology Assessment: Human Health Risks'. We would like to thank Christina Giannakou for the characterization of the nanoparticles and Ilse Gosens for the critical evaluation of the manuscript. 

Published as: Simple in vitro models can predict pulmonary toxicity of silver nanoparticles

Hedwig M. Braakhuis ${ }^{1,2^{*}}$, Christina Giannakou ${ }^{\mathrm{I}, 2}$, Willie J.G.M. Peijnenburg ${ }^{2,3}$, Jolanda Vermeulen $^{2}$, Henk van Loveren ${ }^{1,2}$, Margriet V.D.Z. Park ${ }^{2}$

${ }^{\mathrm{I}}$ Department of Toxicogenomics, Maastricht University, Po Box 6I 6, 6200 MD Maastricht, the Netherlands

${ }^{2}$ National Institute for Public Health and the Environment (RIVM), Po Box I, 3720 BA Bilthoven, the Netherlands

${ }_{3}^{3}$ University Leiden, Centre for Environmental Sciences, Po Box 95 I 8, 2300 RA Leiden, the Netherlands

Nanotoxicology: Accepted Nov 2015 


\section{Use of in vitro models of increasing complexity to predict pulmonary toxicity of silver nanoparticles}




\section{ABSTRACT}

To study the effects of nanomaterials after inhalation, a large number of in vitro lung models have been reported in literature. Although the in vitro models contribute to the reduction of animal studies, insufficient data exists to determine the predictive value of these in vitro models for the in vivo situation. The aim of this study was to determine the correlation between in vitro and in vivo data by comparing the dose metrics of silver nanoparticles in an in vitro lung model of increasing complexity and in an in vivo inhalation study. In vivo, the alveolar dose expressed as particle surface area was found to be the most suitable dose metric to describe the toxicity of silver nanoparticles after inhalation. The results of the present study show that particle surface area is a suitable dose metric to describe the effects of silver nanoparticles when using a simple monolayer of lung epithelial cells. The dose metric shifted from particle surface area to particle mass when adding an increasing number of macrophages. In addition, a co-culture of endothelial cells, epithelial cells and macrophages on a Transwell ${ }^{\circ}$ insert correlated less well to the in vivo results compared to the epithelial monolayer. We conclude that for studying the acute pulmonary toxicity of nanoparticles simple in vitro models using an epithelial monolayer better predict the in vivo response compared to complex co-culture models.

\section{INTRODUCTION}

Nanomaterials are used in an increasing number of products like electronic devices, textiles, sprays, paints and cosmetics (Nanotechnologies, 20 I 4). There are concerns that the increasing use of nanomaterials might lead to health risks for both workers and consumers. Especially inhalation of nanomaterials is of concern since aerosolization may occur during energetic processes such as vortexing, weighing, sonication, mixing and blending (Maynard and Kuempel, 2005). Several in vivo inhalation studies have been performed the past years to determine the effects of nanomaterials after inhalation (Arts et al., 2007, Braakhuis et al., 20I 5a, Braakhuis et al., 20I4a, Gosens et al., 20I4, Grassian et al., 2007, Ho et al., 20 I I, Ma-Hock et al., 20I3, Pauluhn, 20 Io, Sayes et al., 20ro, Sung et al., 2009, Sung et al., 20I rb, Warheit et al., 2007c). These studies provide valuable information on the distribution and effects of the tested nanomaterials after inhalation exposure. However, the use of animals is ethically debatable and it is not feasible to test all different types and varieties of nanomaterials in animal models. Therefore, in vitro models have been developed to study the effects of nanomaterials.

Regarding inhalation exposure, a large number of in vitro lung models have been reported in literature ranging in complexity from a single cell type to co-culture models on a chip (Braakhuis et al., 20I 5 c). Most in vitro lung models use lung epithelial cells as a basis. The most frequently used lung epithelial cells lines are A549, CALU-3, H44I and I6HBEI4O- (I6HBE). Of these cell lines, CALU-3, H44I and I6HBE form tight junctions, but A5 49 cells do not (Lehmann et al., 201 I, Chowdhury et al., 2010, Geys et al., 2006, 
Hermanns et al., 2004, George et al., 201 5 ). To obtain co-culture models, different cell types are added to the basic model, for example endothelial cells, alveolar macrophages, dendritic cells, mast cells, type II alveolar cells, fibroblasts (Brandenberger et al., 20 Ioa, Chowdhury et al., 20 io, dos Santos et al., 20 I , Frieke Kuper et al., 20 I 5, George et al., 20 I 5 , Geys et al., 2006, Hermanns et al., 2004, Huh et al., 20 I0, Madlova et al., 2009, Muller et al., 20 I0, Raemy et al., 20 I , Rothen-Rutishauser et al., 2008).

Although the in vitro models aim to contribute to the reduction of animal studies, it remains unclear how the results of these in vitro models correlate to the in vivo situation and how these results can be used in the risk assessment of nanomaterials. In addition, many in vitro lung models are based on the co-culture of different cell types to mimic more closely the in vivo situation compared to the use of a single cell type. However, only few studies evaluated the predictive value of those more complex co-culture models compared to single cell models. The aim of this study was to determine the correlation between results obtained in an in vitro lung model of increasing complexity compared to results obtained in a previously performed in vivo inhalation study (Braakhuis et al., 20 I 5a). The results of our previous study show that the alveolar dose expressed as particle surface area is a suitable dose metric to describe the toxicity of silver nanoparticles after inhalation (Braakhuis et al., 2015a). By exposing in vitro lung models to silver nanoparticles of different sizes, we can analyse the effects in a similar way and determine if the dose metric found in vivo can also be found in vitro. To test in vitro lung models of increasing complexity, we started with a simple monolayer of human lung epithelial cells (I6HBE). To increase the resemblance of the model to the in vivo situation, we extended the monolayer by adding differentiated human alveolar macrophages (THP-I) in different cell densities. Finally, we tried to mimic the lung-blood barrier by culturing different cell types on a Transwell ${ }^{\circ}$ insert; human lung epithelial cells with alveolar macrophages at the apical side and human umbilical vein endothelial cells (HUVEC) at the basolateral side. After exposure to silver nanoparticles of different sizes, cytotoxicity and the induction of inflammatory cytokines and reactive oxygen species (ROS) were measured in the in vitro models. The results were analysed based on the mass, particle number and surface area dose and compared to the results from the previous in vivo study using silver nanoparticles of different sizes (Braakhuis et al., $2015 \mathrm{a}$ ).

\section{MATERIALS AND METHODS}

\section{Nanomaterials}

BioPure uncoated, spherical silver nanoparticles (NPs) of average nominal diameters of IO, 20, 50, and Ioo $\mathrm{nm}$ were purchased from NanoComposix, Inc. (San Diego, CA, USA). The NPS were supplied in the form of stock dispersions of $\mathrm{I} \mathrm{mg} / \mathrm{ml}$ in aqueous $2 \mathrm{mM}$ citrate. All particles were negative for endotoxin contamination in the limulus amebocyte lysate (LAL) test. 


\section{Nanomaterial characterization}

To confirm the primary particle size of the silver NPs, TEM images were acquired using a Tecnai Io apparatus (Fei, the Netherlands) at an acceleration voltage of $\mathrm{I} 00 \mathrm{kV}$ and a Mega View III digital camera (Soft Imaging System, GmbH, Munchen, Germany). The silver NPs were diluted in $2 \mathrm{mM}$ citrate buffer and droplets of $3 \mu$ l were placed on TEM grids till the fluid was vaporized.

Nanoparticle size distribution was determined in $2 \mathrm{mM}$ citrate buffer and in complete culture medium without foetal calf serum (FCS) in a concentration of I and IO $\mu \mathrm{g} / \mathrm{ml}$ after 5 minutes, 24 hours and 7 days incubation using both the dynamic light scattering (DLS) technique and the centrifugal particle sedimentation (CPS) technique. The DLS measurements were performed using a Malvern Zeta Sizer (Worchestershire, uK). Hydrodynamic diameter was calculated based on the average of Io runs of the intensity weighted Z-average performed at $25^{\circ} \mathrm{C}$ using the cumulative fit function performed in triplicate. The CPS measurements were performed using a CPs Disc Centrifuge Model DC 24000 (CPS Instruments, Inc.) This technique uses centrifugal forces to separate and count particles according to their size. Particle separation, sedimentation and measurement were done in an $8-24 \%(\mathrm{w} / \mathrm{w})$ sucrose density gradient and at disc rotation speed of $24000 \mathrm{rpm}$. Prior to each sample measurement the instrument was calibrated using PVC nanosphere standards $(476 \mathrm{~nm})$. Data collection and analysis were performed using the CPS Disc Centrifuge DC Control System Vi I software.

Silver ion release from the silver NPs was determined using an ion selective electrode. This method is based on the activity of the ions, which can be related to their concentration. First, a concentration range of $\mathrm{AgNO}_{3}$ (ranging from $0.000 \mathrm{I}$ to $0 . \mathrm{I} \mathrm{mM}$, equal to $0.00 \mathrm{IO} 8$ to $1.0787 \mu \mathrm{g} / \mathrm{ml}$ ) was prepared to obtain a standard curve. Next, the dissolution of the silver NPS (Io $\mu \mathrm{g} / \mathrm{ml}$ ) was determined in culture medium without FCS, in artificial lysosomal fluid (prepared according to the article from Stopford et al. (Stopford et al., 2003)) and in a $\mathrm{HNO}_{3}$ solution of $\mathrm{pH} 3.43$ and $\mathrm{pH} 4.60$.

\section{Cells and cell culture conditions}

Human bronchial epithelial cells I6HBEI40- cells (I6HBE) were kindly provided by Dr. Gruenert (university of California, San Francisco, USA) and cultured in DMEM/FI 2 medium supplemented with $5 \%$ FCs, I $00 \mathrm{U} / \mathrm{ml}$ penicillin, IOO $\mu \mathrm{g} / \mathrm{ml}$ streptomycin, $2 \mathrm{mM} \mathrm{L}$-glutamine and $2.5 \mu \mathrm{g} / \mathrm{ml}$ fungizone. Human umbilical vein endothelial cells (HUVEC) were purchased from Life Technologies (Thermo Fisher Scientific Inc., the Netherlands) and cultured in M200 supplemented with low serum growth supplement (LSGS), IOO U/ml penicillin, IO० $\mu \mathrm{g} / \mathrm{ml}$ streptomycin and $0.5 \mu \mathrm{g} / \mathrm{ml}$ fungizone. Human acute monocytic leukemia cells (THP-I) were purchased from American Type Culture Collection (ATCC, Rockville, USA) and cultured in RPMI medium supplemented with IO\% FCS, IOO $\mathrm{U} / \mathrm{ml}$ penicillin, and IO० $\mu \mathrm{g} / \mathrm{ml}$ streptomycin. To differentiate the THP-I 
monocytes into macrophages, the cells were seeded in a density of 500.000 cells $/ \mathrm{ml}$ in the presence of $30 \mathrm{ng} / \mathrm{ml}$ PMA for 5 subsequent days. At day 5 , the medium was removed and replaced by full culture medium for 3 days. All culture media and supplements were purchased from Life Technologies (Thermo Fisher Scientific Inc., the Netherlands).

The cells were incubated at $37{ }^{\circ} \mathrm{C}$ in a $100 \%$ humidified atmosphere containing $5 \%$ $\mathrm{CO}_{2}$. When confluent, the epithelial and endothelial cells were released enzymatically (0.05\% trypsin-EDTA) and transferred to a new culture flask. The medium was renewed every 2-3 days.

\section{Preparation of the lung models}

The simple lung model consisted of a monolayer of I6HBE cells cultured in a density of I00.000 cells $/ \mathrm{cm}^{2}$. The monolayer was cultured for 7 days prior to exposure, to give the I6HBE cells the opportunity to form a tight barrier. To mimic the in vivo situation more closely, differentiated THP-I cells were added on top of the I6HBE cells (cultured in a density of I00.000 cells $/ \mathrm{cm}^{2}$ for 7 days) in different cell densities: $3 . \mathrm{I} 25,6.250$, I2.500, $25.000,50.000$ and $100.000 \mathrm{cells} / \mathrm{cm}^{2}$. These densities resulted in a ratio between I6HBE and THP-I cells of I60:I, 80:I, 40:I, 20:I, IO:I, and 5:I. Both the I6HBE monolayer and the co-culture with THP-I cells were cultured in 96-wells plates with a growth area of $0.33 \mathrm{~cm}^{2}$. The complete lung model, consisting of three different cell types, was prepared on non-coated polyester Transwell ${ }^{\ominus}$ insert membranes with $0.4 \mu \mathrm{m}$ pores and a growth area of $0.33 \mathrm{~cm}^{2}$ (inserts fit in a 24-wells plate). HUvEC cells were seeded in a density of $50.000 \mathrm{cells} / \mathrm{cm}^{2}$ on the basolateral side of the Transwell ${ }^{\circ}$ inserts. After 2 hours, the inserts were returned to their original orientation and I $6 \mathrm{HBE}$ cells were seeded in a density of 100.000 cells $/ \mathrm{cm}^{2}$ on the apical side. After 7 days, THP-I macrophages were added to the bi-culture model at the apical side in a density of $50.000 \mathrm{cell} / \mathrm{cm}^{2}$ (I THP-I cell per IO I 6 HBE cells).

\section{Measurements of transepithelial electrical resistance (TEER)}

Transepithelial electrical resistance (TEER) was measured using the Evom2 Voltohmmeter supplemented with an EndOhm-6 chamber (World Precision Instruments, Inc., FL, USA). The TEER values were corrected for the resistance of a cell-free Transwell ${ }^{\circ}$ insert and for the surface area of the insert.

\section{Metabolic activity}

Cell metabolic activity was evaluated using the wST-I cell Proliferation Reagent (Roche, Almere, the Netherlands). The metabolic conversion of WST-I by the cells and the ability of silver NPS to scatter or absorb light or to interfere with the WST-I reagent were assessed in 96-wells plates with the I6HBE alone, in 96-wells plates with a co-culture of I6HBE and THP-I and in the tri-culture model on Transwell ${ }^{\circ}$ inserts with a co-culture 
of HUVEC, I $6 \mathrm{HBE}$ and THP-I according to methods described earlier. Briefly, cells were exposed to silver NPS for 24 hours and incubated with WST-I reagent for the last 3 hours of the exposure. Absorbance was measured using a SpectraMax I 90 scanning multiwell spectrophotometer (Molecular Devices, Sunnyvale, USA) at a wavelength of $440 \mathrm{~nm}$ and a reference wavelength of $620 \mathrm{~nm}$. The absorbance of silver NPS in tissue culture medium, measured in the absence of cells, was subtracted from the total absorbance of the nanoparticle treated cells.

\section{Acellular reactive oxygen species}

The potential of the silver NPs to generate hydroxyl radical formation was evaluated using an Electron Spin Resonance (ESR) spectroscopy method. Silver NPs were incubated with O. I $\mathrm{M} \mathrm{H}_{2} \mathrm{O}_{2}$ and $25 \mathrm{mM}$ of the spin trap 5,5-dimethyl-I-pyrolline N-oxide (DMPO, Sigma). Samples of this solution were taken up in glass capillaries ( $50 \mu$, Hirschmann Ringcaps, Hirschmann Laborgeräte GmbH \& Co, Eberstadt, Germany) after an incubation of I $5 \mathrm{~min}$ at $37^{\circ} \mathrm{C}$. After sealing, the capillary was immediately placed in the resonator of the ESR spectrometer (Magnettech MiniScope Ms 400, Freiberg Instruments GmbH, Freiberg, Germany). ESR spectra were recorded at room temperature.

Instrumental conditions for the recorded spectra were as follows: magnetic field, 3410 G; sweep width, Ioo G; modulation amplitude, 2 G; receiver gain, 9 x IO $^{2}$; scan time, $30 \mathrm{~s}$; number of scans, 3. Quantification of the characteristic DMPO-trapped hydroxyl radical signal (4 peaks splitting constant I $4.9 \mathrm{G}$, surface pattern I:2:2:I) was performed using MiniScopeControl v2.5.I (Magnettech GmbH, Berlin, Germany).

\section{Cellular reactive oxygen species}

Cellular generation of ROs in the lung model after exposure to $0.03-30 \mu \mathrm{g} / \mathrm{cm}^{2}$ silver NPS was measured using the $\mathrm{H}_{2}$ DCF-DA assay in 96 -well plates. Cells were washed with Hanks' balanced salt solution (HBss) and loaded with Iо $\mu \mathrm{M} \mathrm{H}_{2}$ DCF-DA probe (Molecular Probes, Life Technologies, Oregon, USA). After $45 \mathrm{~min}$, cells were washed with HBSs and fluorescence was measured in HBss $(t=0)$ at an excitation wavelength of $485 \mathrm{~nm}$ and an emission wavelength of $535 \mathrm{~nm}$ using a Tristar ${ }^{2}$ Multimode Reader LB 942 (Berthold Technologies, Belgium). Subsequently, cells were exposed to silver NPs in culture medium without phenol red and without FCS. I $\% \mathrm{H}_{2} \mathrm{O}_{2}$ was used as a positive control. Fluorescence was measured at I hour, 4 hours and 24 hours after exposure. The fluorescence of silver NPs alone, measured in the absence of cells, was subtracted from the total absorbance of the nanoparticle treated cells. Results were obtained from three independent experiments.

\section{Inflammation markers}

Release of inflammation markers MCP-I and IL-8 were measured in supernatant of cells after 24 hours of exposure to silver NPS $\left(0.03-30 \mu \mathrm{g} / \mathrm{cm}^{2}\right)$ using ELISA (eBioscience, 
Inc.). Samples were collected from three independent experiments. Cytokine analysis was performed according to the manufacturer's instructions. Absorbance was measured using a SpectraMax 190 scanning multiwell spectrophotometer (Molecular Devices, Sunnyvale, USA) at a wavelength of $450 \mathrm{~nm}$ and a reference wavelength of $570 \mathrm{~nm}$.

\section{Cellular uptake of silver nanoparticles}

To investigate the uptake and intracellular fate of silver NPs, differentiated THP-I cells were cultured on Aclar pieces in a 6-wells plate. The THP-I cells were exposed to silver NPS of $20 \mathrm{~nm}$ at a concentration of $3 \mu \mathrm{g} / \mathrm{cm}^{2}$ (equals Io $\mu \mathrm{g} / \mathrm{ml}$ ) for 6 hours and fixed either directly or after a resting period of $\mathrm{I} 8$ hours. Cells were fixed with half-strength Karnovsky fixative (0.08 M Sodium-cacodylate buffer, $2.5 \%$ glutaraldehyde, $0.025 \mathrm{mM}$ $\mathrm{CaCl}_{2}, 0.05 \mathrm{mM} \mathrm{MgCl}_{2}$ ) overnight for electron microscopy. The next day, the fixative was replaced with $2 \%$ para-formaldehyde in sodium-cacodylate buffer for storage. The cells were post-fixed by osmium potassium ferro-cyanide solution (o. I M sodiumcacodylate buffer, I\% osmium tetra-oxide, I. $\% \%$ potassium ferro-cyanide). Next, the Aclar pieces containing cells were dehydrated in ethanol series and subsequently embedded in epon. Before the final embedding in freshly prepared epon, the Aclar pieces were cut into small pieces and put in a mold. The epon was polymerized at 60 ${ }^{\circ} \mathrm{C}$. The epon blocks were trimmed prior to sectioning. During sectioning, ultrathin sections of $70 \mathrm{~nm}$ were sectioned and put on $3 \mathrm{~mm}$ hexagonal copper grids coated with a formvarfilm. The sections were analysed by transmission electron microscopy (TEM) (Tecnai Io, IookV, fEI, Eindhoven, the Netherlands). To investigate whether the silver NPs dissolved intracellularly, the exact particle size of about 400 silver NPS was determined.

\section{Statistical analysis}

To derive an appropriate dose metric, we used the method described by Delmaar et al. 2015 (Delmaar et al., 2015). According to this method, dose response data from experiments are analysed to determine, for each of the investigated NPs, the number of NPs per unit of volume of the test medium needed to induce a predefined response. These doses are referred to as "equi-response doses". For example, for the silver NPs in this study, the number of particles needed to induce a $20 \%$ decrease in cell metabolism is $N 1$ particles $/ \mathrm{ml}$ for particles with diameter $d 1$ and $N 2$ particles $/ \mathrm{ml}$ for particles with diameter $d 2$. Then the doses ' $N 1$ particles $/ \mathrm{ml}$ of diameter $d 1$ ' and ' $N 2$ particles $/ \mathrm{ml}$ of diameter $d 2$ ' are equi-response doses. By plotting the diameter of the particles on the $\mathrm{x}$-axis and the number of particles that induce a predefined response on the $\mathrm{y}$-axis, an equi-response curve can be constructed. This equi-response curve, constructed from dose-response data, can be used to determine the appropriate dose metric. In the case that the equi-response curve is used to test whether any of the simple dose metrics, administered surface area, volume or number of particles is appropriate, the equiresponse curve on a double log scale should be a straight line. If the slope of the equi- 
response curve in the $\log (N)$ versus $\log (d)$ plot derived from experimental data equals -2 , surface area is an adequate description of the dose. If, on the other hand, the slope equals -3 , volume is an adequate dose description, and if it equals $\mathrm{o}$, the adequate dose description is total number of particles. Of course there is the possibility that the slope of the equi-response curve may not equal $-2,-3$ or 0 at all. In this case, the function of the curve may still be used to derive an adequate dose metric consisting of both parameters $N$ and $d$ (Delmaar et al., 201 5). A more detailed explanation of this method and the rationale behind it is described in Delmaar et al. 2015 .

All experimental data were combined in one dataset, which was analysed using the dose-response modelling software PROAST (RIVM, Bilthoven, the Netherlands) (Slob, 2002, RIVM, 20I4), according to methods described earlier (Park et al., 2009). PROAST is benchmark dose software that can be used for dose-response analysis (Barlow et al., 2009, EFSA, 2009). Briefly, the PROAST software selects the optimal data fitting model from an exponential family of models using the likelihood-ratio criterion (Slob, 2002). For the comparison of particles, the particle identification (I0, 20, 50, I00) was defined as a covariate for the slope $b$, which reflects the potency of the administered agent. A $\mathrm{p}$-value of $<0.05$ for the associated likelihood-ratio test was considered as a statistically significant effect of particle size. The potential differences between particle sizes were further quantified by the critical effect dose of silver $\mathrm{NPS}_{\mathrm{CED}}{ }_{20}$, defined as the (nominal) dose resulting in a $20 \%$ response compared to the control group. We chose a critical effect size of $20 \%$ based on the magnitude of effect in previous studies with ambient particulate matter or NPs (Stoeger et al., 2006, Gosens et al., 20 I 4, Gerlofs-Nijland et al., 2007) and expert judgement on physiologically relevant changes. In addition, the data was analysed by the Kruskal Wallis nonparametric test (Graphpad Prism). Statistical significance is indicated with $\mathrm{a}^{*}(p=<0.05)$. In all graphs, error bars represent the standard deviation of the mean.

\section{RESULTS}

\section{Nanomaterial characterization}

The primary particle size of I0, 20, 50 and $100 \mathrm{~nm}$ of the silver NPs was confirmed by TEM (figure I). The agglomerate particle size in citrate buffer and culture medium without FCS was measured using both DLS and CPS (figure 2). Using DLS, a small amount of larger agglomerates can dominate the intensity distribution. In addition, for the IO $\mathrm{nm}$ silver NPs, the polydispersity index (PdI) was sometimes greater than 0.7 indicating that the sample was probably not suitable for the DLS technique. CPS does not have these limitations when measuring the agglomerate size of silver NPs. Therefore, we assume the CPS measurements are more accurate compared to the DLS measurements. Using DLS, the mean agglomerate size of the silver NPs in $2 \mathrm{mM}$ citrate buffer (buffer) was 32.3 , $22.2,300.2$, and $885.1 \mathrm{~nm}$ for the I0, 20, 50 and $100 \mathrm{~nm}$ silver NPs, respectively. In 
culture medium without FCS (medium), the agglomerate size was I997, I4 I 6.7, I455.7, I054.9 $\mathrm{nm}$, respectively. Using CPS, the median agglomerate size of the silver NPS in buffer was 9.62, $17.6,41.0$, and $82.56 \mathrm{~nm}$ for the 10, 20, 50, and I00 nm silver NPs, respectively. In medium, the median agglomerate size was 77.86, 92.17, 106.82, and I65.7, respectively. The agglomerate size in medium was stable over time. After 24 hours incubation, the agglomerate particles size of the 10, 20, 50 and $100 \mathrm{~nm}$ silver NPs was 74.42, 71.93, I24.67, and $199.04 \mathrm{~nm}$, respectively. After 7 days of incubation, the agglomerate size was I I0.53, 92.4, I 35.4, and I86.66, respectively. There was no decrease in agglomerate size that is indicative of the release of silver ions. There were no differences in the size distribution measurements between I and Io $\mu \mathrm{g} / \mathrm{ml}$ concentrations.

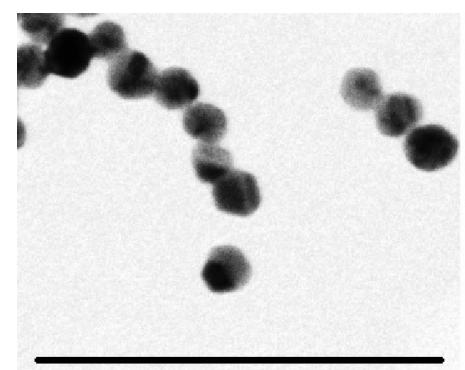

$100 \mathrm{~nm}$

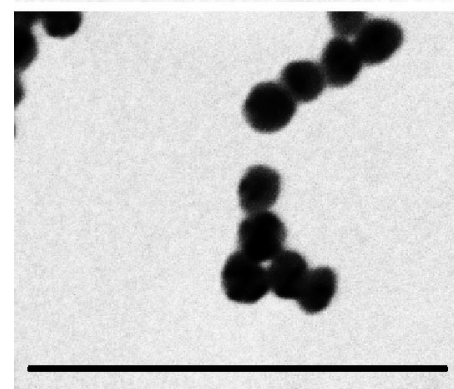

$500 \mathrm{~nm}$

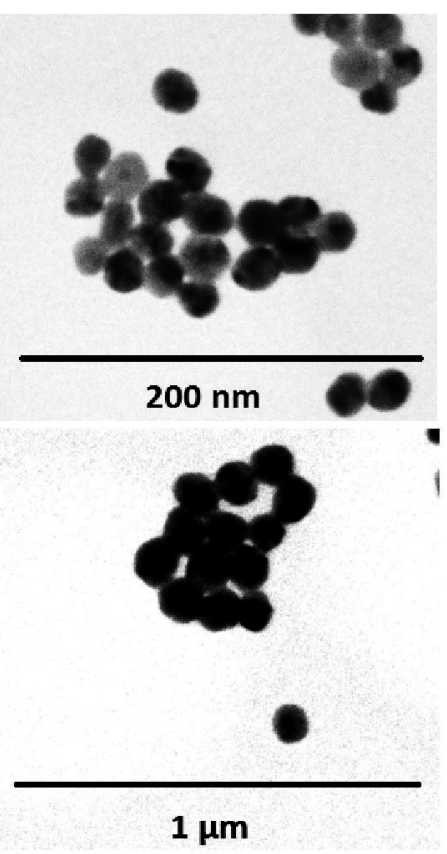

Figure 1. TEM pictures of the silver NPS of $10 \mathrm{~nm}$ (top left), $20 \mathrm{~nm}$ (top right), $50 \mathrm{~nm}$ (bottom left) and $100 \mathrm{~nm}$ (bottom right). The silver NPs did not release ions in culture medium or artificial lysosomal fluid (ALF) of $\mathrm{pH} 4.6$ up to 24 hours as measured using the ion selective electrode (data not shown). Visible precipitates containing silver formed after incubation of the silver NPS with ALF. They did dissolve completely in an acidic $\mathrm{HNO}_{3}$ solution of $\mathrm{pH} 3.43$ within $\mathrm{I}_{5}$ minutes and in an acidic $\mathrm{HNO}_{3}$ solution of $\mathrm{pH}$ 4.6 within 5 hours. 

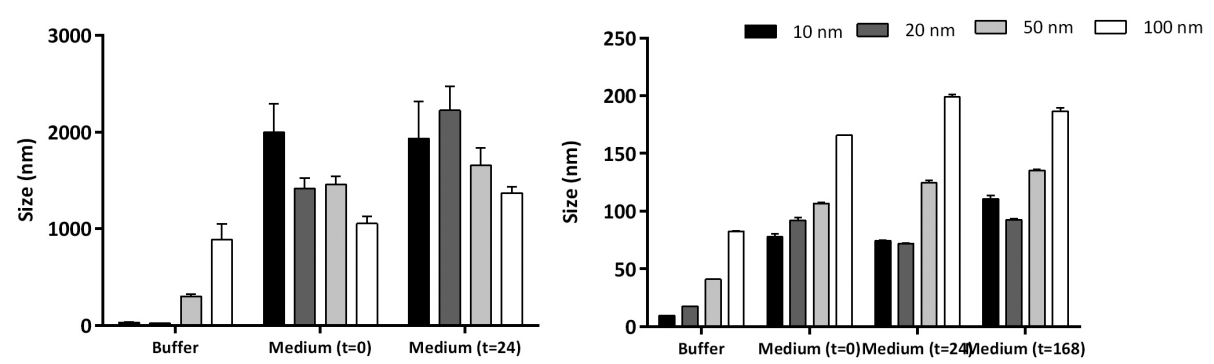

Figure 2. Median agglomerate particle size of $10,20,50$ and $100 \mathrm{~nm}$ silver NPs in $2 \mathrm{mM}$ citrate buffer and in complete culture medium without $\mathrm{FCS}$ at $\mathrm{t}=0, \mathrm{t}=24$ hours and $\mathrm{t}=7$ days, measured using dynamic light scattering (DLS) (left graph) and using centrifugal particle sedimentation (CPS) (right graph). Data is derived from three individual experiments and presented as mean with a standard deviation. The silver NPs agglomerate in culture medium. The agglomerate size is similar over time.

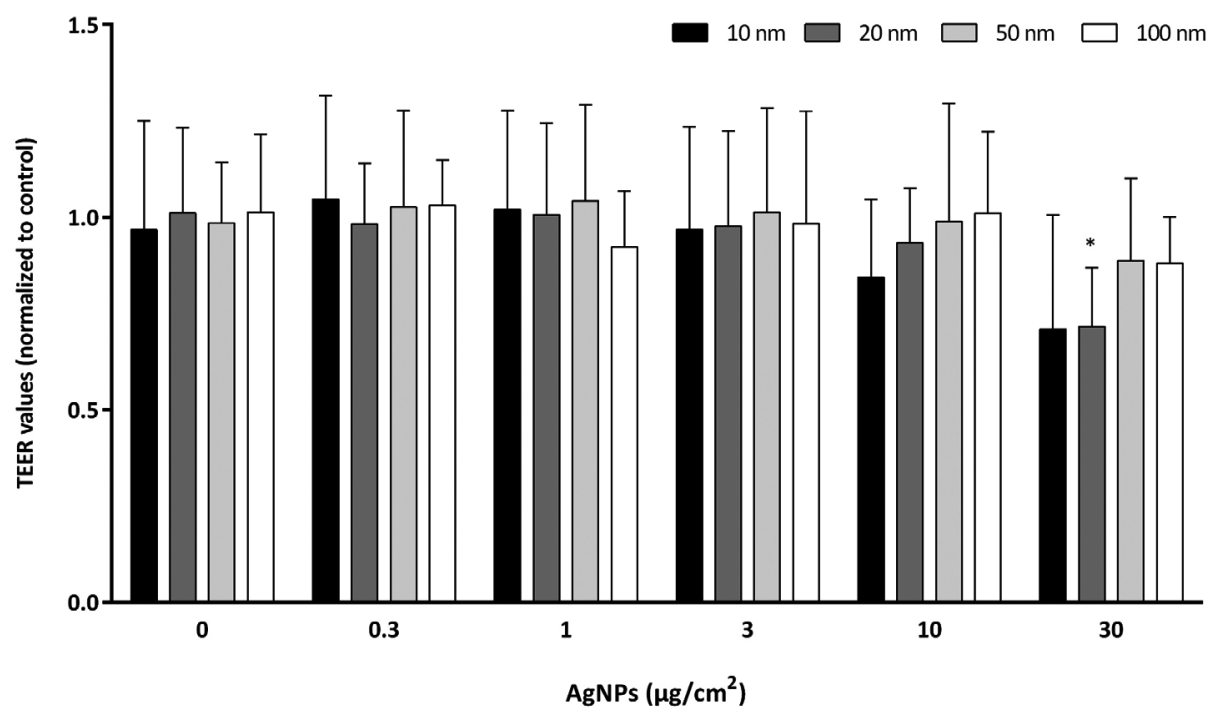

Figure 3. Trans-epithelial electrical resistance (TEER) in a co-culture of endothelial cells, epithelial cells and alveolar macrophages cultured on Transwell ${ }^{\circledR}$ inserts after 24 hours exposure to silver NPS of four different sizes. Exposure to silver NPS resulted in a slight decrease in TEER, however not statistically significant for the 10, 50 and $100 \mathrm{~nm}$ NPS. For the $20 \mathrm{~nm}$ silver NPS, the TEER was significantly decreased at the highest exposure concentration $(p<0.05)$. 


\section{Metabolic activity}

In the monolayer of I6HBE, exposure to silver NPs for 24 hours resulted in a significantly decreased metabolic activity for all particle sizes at concentrations of $\mathrm{Io} \mu \mathrm{g} / \mathrm{cm}^{2}$ (equals $30 \mu \mathrm{g} / \mathrm{ml}$ ) and above. At the concentration of $30 \mu \mathrm{g} / \mathrm{cm}^{2}$ (equals Ioo $\mu \mathrm{g} / \mathrm{ml}$ ), there was a size-dependent decrease in metabolic activity (figure 4). When analysing the results based on mass, particle number and surface area, the dose-response curves of the different particle sizes almost completely overlapped when the exposure was expressed on the basis of the particle surface area (figure 5).

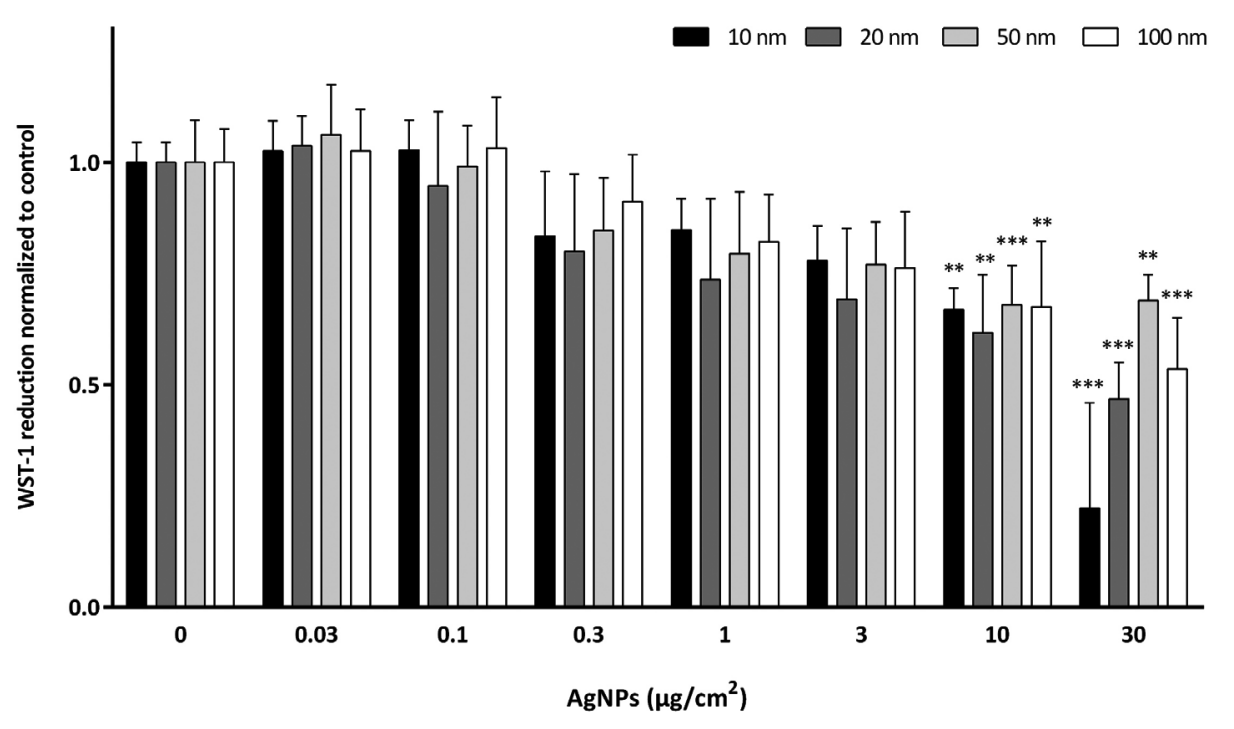

Figure 4. Metabolic activity of $16 \mathrm{HBE}$ cells after 24 hours exposure to silver NPS of $10,20,50$ and $100 \mathrm{~nm}$. Bars represent mean values with their standard deviation. After exposure to 10 $\mu \mathrm{g} / \mathrm{cm}^{2}$ and $30 \mu \mathrm{g} / \mathrm{cm}^{2}$ of $10,20,50$ and $100 \mathrm{~nm}$ silver NPS, the metabolic activity significantly decreased $\left({ }^{* *}=p<0.01,{ }^{* * *}=p<0.001\right)$.

For the co-culture of I6HBE and different densities of THP-I, we did the same analysis. The results showed that with increasing density of the THP-I cells the dose-response curves of the different sizes of silver NPs shifted from overlapping when the dose was expressed as surface area to overlapping when the dose was expressed as mass. By analysing all dose-response curves using the method described by Delmaar et al. (2015), we could construct equi-response curves for the different densities of THP-I cells co-cultured on I6HBE. The slope of the equi-response curves gives information about the appropriate dose metric and shows that for $16 \mathrm{HBE}$ alone surface area seems the most appropriate dose metric (slope of -I.99, $\mathrm{R}^{2}$ of 0.9I). There is a shift from surface area towards mass as the appropriate dose metric with increasing density of THP-I in the co-culture (table I). For 
the complete lung model consisting of endothelial cells, epithelial cells and macrophages cultured on insert membranes, the dose-response curves overlapped mostly when the dose was expressed as mass (slope of $-2.65, \mathrm{R}^{2}$ of 0.99 ) as shown in table 3 .

Viability of $16 \mathrm{HBE}$ after exposure to silver nanoparticles
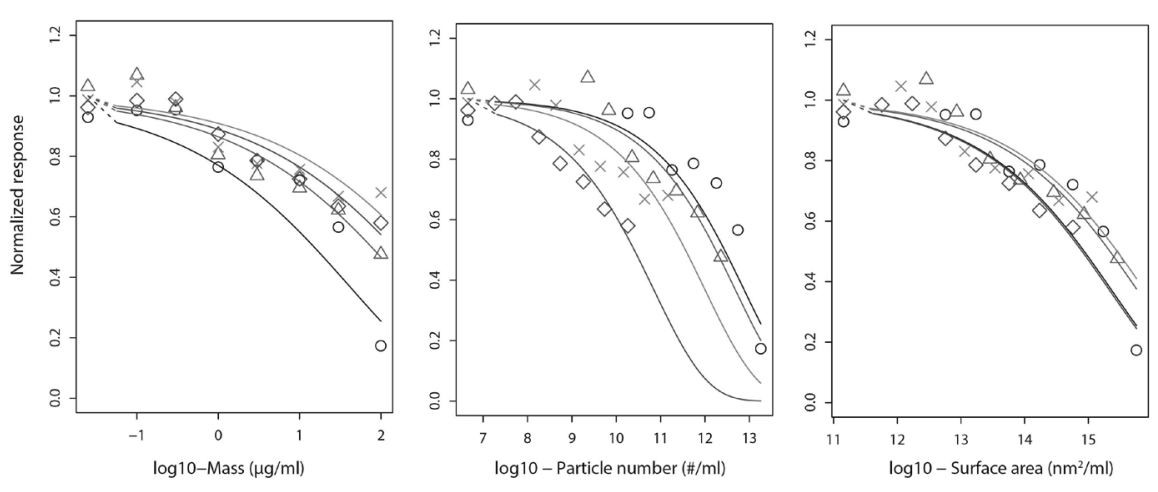

Figure 5. Metabolic activity of $16 \mathrm{HBE}$ cells after 24 hours exposure to $10,20,50$ and $100 \mathrm{~nm}$ silver NPS. Data is shown based on the mass (left), particle number (middle) and surface area dose (right). The symbols represent the mean values of at least three independent replicates.

Table 1. Summary of the slope of the equi-response curves in a $\log (N)$ versus $\log (d)$ plot, determined from the dose-response curves of viability of $16 \mathrm{HBE}$ cells co-cultured with different cell densities of THP-1 cells after exposure to silver NPS of different sizes for 24 hours.

\begin{tabular}{|c|c|c|c|}
\hline Cells & Slope & $\mathrm{R}^{2}$ & Dose metric \\
\hline $16 \mathrm{HBE}$ & $-1.99(-2.67$ to -1.26$)$ & 0.91 & Surface area \\
\hline 16HBE and THP-1 (3125 cells/cm ) & $-2.30(-2.41$ to -2.19$)$ & 0.98 & \\
\hline 16HBE and THP-1 (6250 cells/cm $\left.{ }^{2}\right)$ & $-2.39(-2.44$ to -2.33$)$ & 0.98 & \\
\hline 16HBE and THP-1 (12500 cells/cm $\left.{ }^{2}\right)$ & $-2.39(-2.91$ to -1.87$)$ & 0.94 & \\
\hline $16 \mathrm{HBE}$ and THP-1 (25000 cells/cm $\left.{ }^{2}\right)$ & $-2.49(-3.08$ to -1.89$)$ & 0.93 & \\
\hline 16HBE and THP-1 (50000 cells/cm $\left.{ }^{2}\right)$ & $-2.81(-3.04$ to -2.59$)$ & 0.98 & $v$ \\
\hline $16 \mathrm{HBE}$ and THP-1 (100000 cells/cm $\left.{ }^{2}\right)$ & $-2.74(-2.88$ to -2.60$)$ & 0.99 & Mass \\
\hline
\end{tabular}




\section{Acellular generation of reactive oxygen species}

The acellular generation of ROs in both buffer and medium was investigated by means of electron spin resonance (ESR). The IO and $20 \mathrm{~nm}$ silver NPS showed an additional $\mathrm{H}_{2} \mathrm{O}_{2}-$ induced formation of hydroxyl radicals compared to buffer and medium alone, while there was no additional $\mathrm{H}_{2} \mathrm{O}_{2}$-induced formation of hydroxyl radicals by 50 and $\mathrm{I} 0 \mathrm{Omm}$ silver NPs in buffer or medium (table 2).

Table 2. Acellular induction of reactive oxygen species by silver NPS using Electron Spin Resonance (ESR) spectroscopy in combination with the spin trapping technique.

\begin{tabular}{lll}
\hline $\begin{array}{l}\text { Silver } \\
\text { nanoparticles }\end{array}$ & $2 \mathrm{mM}$ citrate buffer & $\begin{array}{l}\text { Culture medium without } \\
\text { FCS }\end{array}$ \\
$10 \mathrm{~nm}$ & 1.75 & 1.93 \\
$20 \mathrm{~nm}$ & 1.33 & 2.12 \\
$50 \mathrm{~nm}$ & 0.92 & 1.10 \\
$100 \mathrm{~nm}$ & 0.91 & 1.15 \\
\hline
\end{tabular}

\section{Cellular generation of reactive oxygen species}

The viability assay in I6HBE cells showed that at concentrations of Io and $30 \mu \mathrm{g} / \mathrm{cm}^{2}$, the metabolic activity was significantly decreased. Therefore, we performed an analysis of Ros generation for concentrations up to $3 \mu \mathrm{g} / \mathrm{cm}^{2}$, and excluded the two highest concentrations. As measured by the $\mathrm{H}_{2} \mathrm{DCF}-\mathrm{DA}$ assay, exposure of I6HBE epithelial cells to silver NPS resulted in a doubling in the generation of ROs for all particle sizes already at the lowest concentration tested of $0.03 \mu \mathrm{g} / \mathrm{cm}^{2}$. With increasing concentrations, Ros generation did not further increase; there was no dose-response relation (figure 6). Compared to the positive control $\mathrm{H}_{2} \mathrm{O}_{2}$, which gave about a 20 -fold increase in ROs generation, ROs production in I6HBE cells was overall low. Exposure of THP-I macrophages to silver NPS also resulted in a doubling of ROs generation, however this occurred at higher silver nanoparticle concentrations compared to the I6HBE cells. The Io nm silver NPS caused a slightly higher generation of ROS of about 3-fold compared to the controls at a concentration of $\mathrm{I}$ and $3 \mu \mathrm{g} / \mathrm{cm}^{2}$. For the 20,50 and $\mathrm{I} 00 \mathrm{~nm}$ silver NPs, there was no dose-response relation between the silver nanoparticle concentration and ROS generation. In the co-culture of I6HBE and THP-I, exposure to silver NPS resulted in an increase in the generation of Ros for all particle sizes already at the lowest concentration tested of $0.03 \mu \mathrm{g} / \mathrm{cm}^{2}$. Similar to the I6 $\mathrm{HBE}$ monolayer, ROS generation did not further increase with increasing silver nanoparticle concentrations. Because of the lack of dose-response relations between the silver NPS and Ros generation, we could not use these results to determine an appropriate dose metric. 

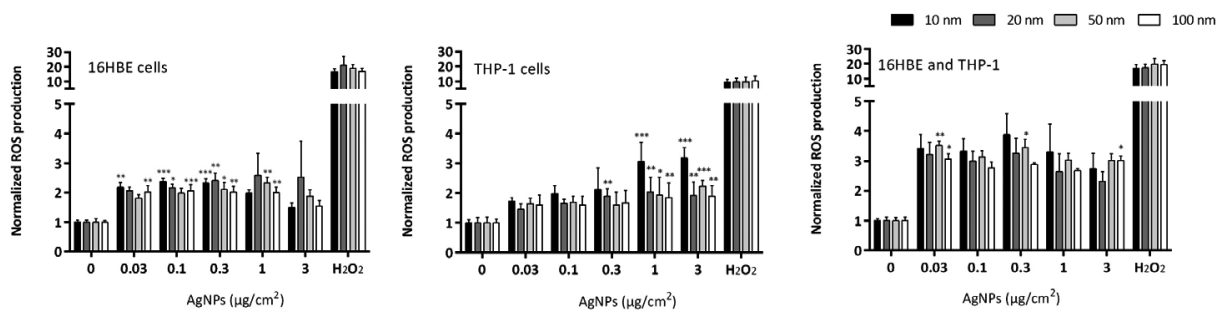

Figure 6. ROS production in $16 \mathrm{HBE}$ epithelial cells (left), differentiated THP-1 macrophages (middle), and a co-culture of $16 \mathrm{HBE}$ and THP-1 (right) after exposure to silver NPS of different sizes. In the $16 \mathrm{HBE}$ cells, the lowest concentration of $0.03 \mu \mathrm{g} / \mathrm{cm}^{2}$ induced a doubling in ROS production for all particle sizes, which did not increase further upon higher exposures. In the THP-1 macrophages, the ROs generation increased most for the smallest particle size of $10 \mathrm{~nm}$. In the co-culture, the lowest concentration of $0.03 \mu \mathrm{g} / \mathrm{cm}^{2}$ induced an increase in ROS production for all particle sizes, which did not increase further upon higher exposures. $\left(^{*}=\right.$ $\left.p<0.05,{ }^{* *}=p<0.01,{ }^{* * *}=p<0.001\right)$.

MCP-1 production in 16HBE after exposure to silver nanoparticles

O $10 \mathrm{~nm}$ silver $\triangle 20 \mathrm{~nm}$ silver $\times 50 \mathrm{~nm}$ silver $\diamond 100 \mathrm{~nm}$ silver
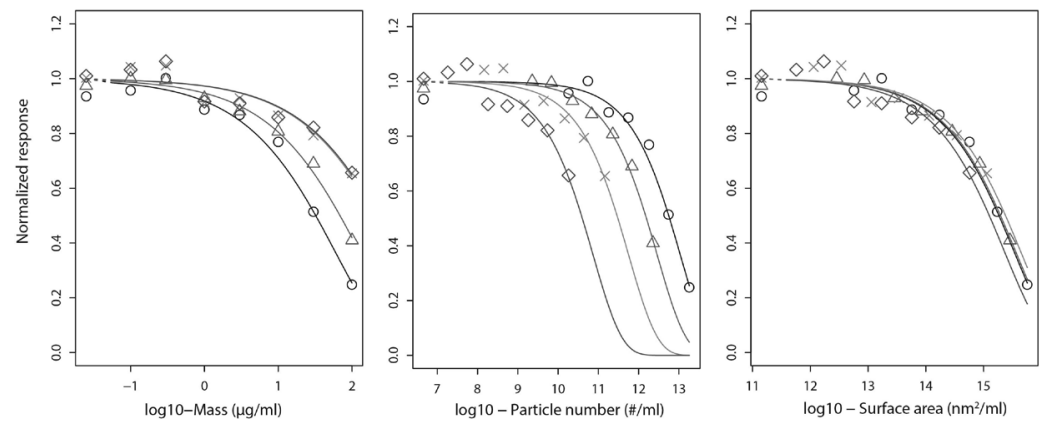

IL-8 production of $16 \mathrm{HBE}$ after exposure to silver nanoparticles

○ $10 \mathrm{~nm}$ silver $\triangle 20 \mathrm{~nm}$ silver $\times 50 \mathrm{~nm}$ silver $\diamond 100 \mathrm{~nm}$ silver
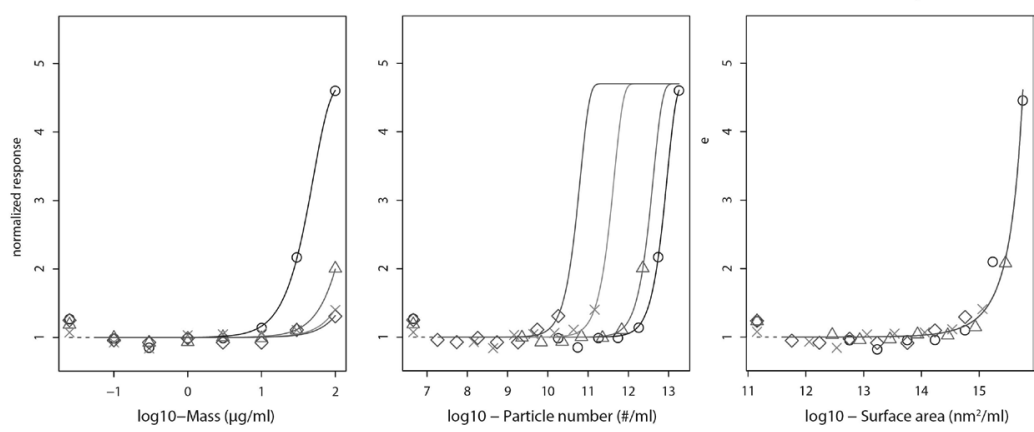

Figure 7. MCP-1 (top) and IL-8 (bottom) production of $16 \mathrm{HBE}$ cells after 24 hours exposure to $10,20,50$ and $100 \mathrm{~nm}$ silver NPS. Data is shown based on the mass (left), particle number (middle) and surface area dose (right). The symbols represent the mean values of at least three independent replicates. 


\section{Inflammatory cytokines}

MCP-I and IL- 8 were measured in the supernatant after 24 hours exposure to IO, 20, 50 , and $100 \mathrm{~nm}$ silver NPs. In the monolayer of I $6 \mathrm{HBE}$, exposure to silver NPs for 24 hours resulted in a dose-dependent decrease in MCP-I production and a dose-dependent increase in IL-8. When analysing the results based on mass, particle number and surface area, the dose-response curves of the different particle sizes almost completely overlapped when the exposure was expressed based on the particle surface area for both MCP-I and IL-8 (figure 7). For the co-culture of HUVEC, I6 HBE and THP-I on Transwell ${ }^{\circ}$ inserts, we did the same analysis. The results showed again a dose-dependent decrease and increase in MCP-I and IL-8, respectively. However, when expressed as mass, particle number or surface area, none of the dose metrics resulted in overlapping dose-response curves of the different sizes of silver NPs (data not shown). By analysing all dose response curves using the method described by Delmaar et al. (2015), we could construct equi-response curves for the monolayer of $16 \mathrm{HBE}$ and the complete lung model on an insert. For I $6 \mathrm{HBE}$ alone surface area seems the most appropriate dose metric. For the complete lung model cultured on Transwell ${ }^{\circ}$ inserts, the appropriate dose metric is probably mass for MCP-I production, and surface area for IL- 8 production. However, for both MCP-I and IL-8 production in the complete lung model, the $\mathrm{R}^{2}$ of the equi-response curve is lower compared to the I6HBE monolayer and the confidence intervals are higher, indicating variation and uncertainty in the data (table 3 ).

Table 3. Summary of the slope of the equi-response curves in a $\log (N)$ versus $\log (d)$ plot, determined from the dose-response curves of WST-1 reduction and MCP-1 and IL-8 production in $16 \mathrm{HBE}$ cells and in co-cultures of HUVEC, $16 \mathrm{HBE}$, and THP-1 after exposure to silver NPS of different sizes for 24 hours.

\begin{tabular}{lllll}
\hline Measurement & Cells & Slope & $\mathbf{R}^{2}$ & Dose metric \\
\hline WST-1 reduction & 16HBE & $-1.99(-2.67$ to -1.26$)$ & 0.91 & Surface area \\
WST-1 reduction & HUVEC, 16HBE, and THP-1 on insert & $-2.65(-2.96$ to -2.37$)$ & 0.99 & Mass \\
MCP-1 production & 16HBE & $-2.11(-2.31$ to -1.91$)$ & 0.99 & Surface area \\
MCP-1 production & HUVEC, 16HBE, and THP-1 on insert & $-3.08(-4.17$ to -1.99$)$ & 0.74 & Mass \\
IL-8 production & 16HBE & $-2.19(-2.35$ to -2.05$)$ & 0.98 & Surface area \\
IL-8 production & HUVEC, 16HBE, and THP-1 on insert & $-2.05(-2.53$ to -1.55$)$ & 0.80 & Surface area \\
\hline
\end{tabular}

\section{Cellular uptake of silver nanoparticles}

During the exposures of I $6 \mathrm{HBE}$ monolayers and the co-cultures of I6HBE with THP-I macrophages, we examined the cells using light microscopy. In the I6HBE monolayer, silver NPs agglomerated in the culture medium and deposited on top of the monolayer. 
In the co-culture, silver NPS were taken up by the alveolar macrophages and fewer NPS deposited on top of the epithelial cell layer (figure 8). To examine the uptake and intracellular fate of silver NPs more closely, THP-I macrophages were exposed to $20 \mathrm{~nm}$ silver NPS $\left(3 \mu \mathrm{g} / \mathrm{cm}^{2}\right.$, equals Io $\left.\mu \mathrm{g} / \mathrm{ml}\right)$ for 6 hours and fixated for TEM analysis either immediately after exposure or after a rest period of I 8 hours. TEM analysis showed that most silver NPS were taken up in vesicles in the cytoplasm during the 6 hours exposure (figure 9). After 6 hours exposure, the average primary particle size was I $8.3 \mathrm{~nm}$ (I4.422. I nm). After the I 8 hours resting period, there was no sign of dissolution of the silver NPs. The average primary particles size was I $8.2 \mathrm{~nm}(\mathrm{I} 5.7-20.8 \mathrm{~nm})$.
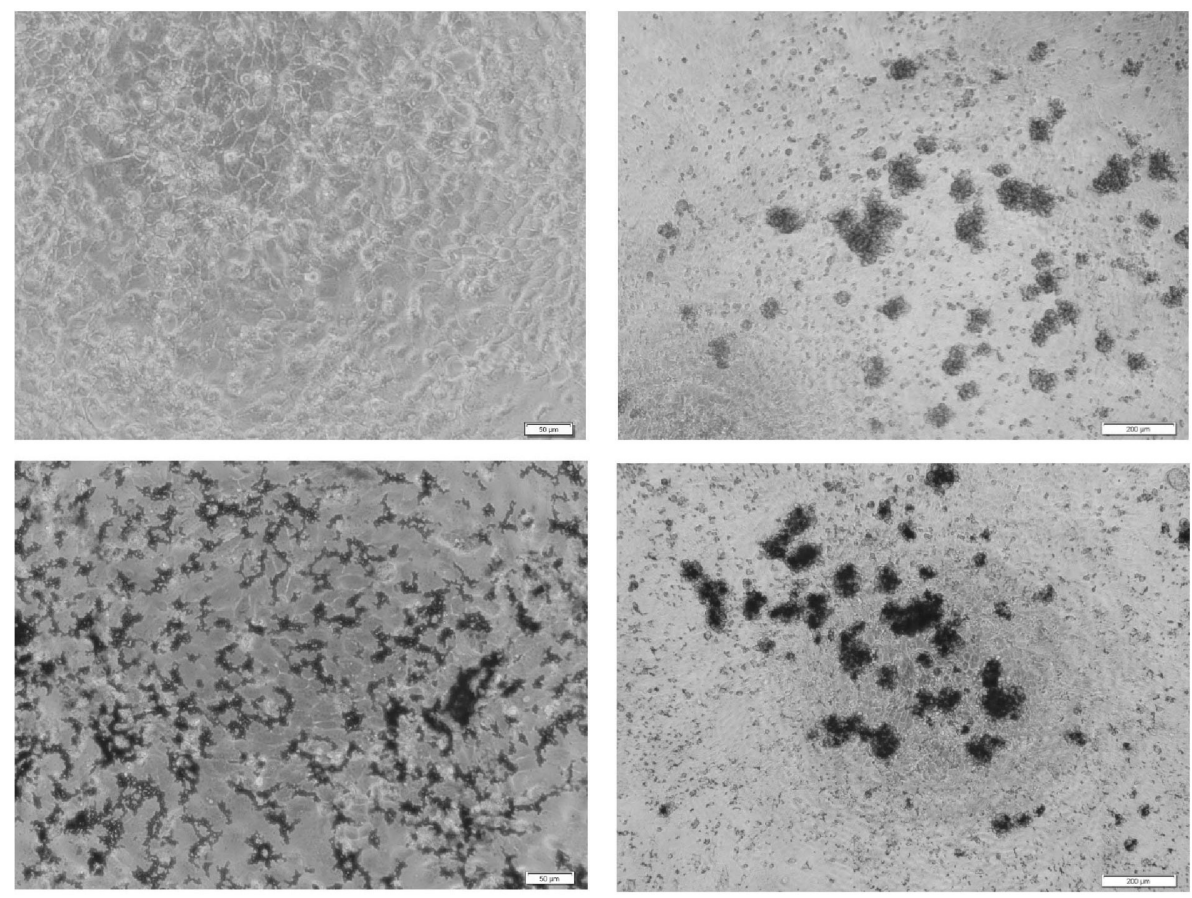

Figure 8. Light microscopy pictures of an unexposed monolayer of $16 \mathrm{HBE}$ cells (top left), an unexposed co-culture of $16 \mathrm{HBE}$ and THP-1 (top right), a monolayer of $16 \mathrm{HBE}$ exposed to $3 \mu \mathrm{g} /$ $\mathrm{cm}^{2} 20 \mathrm{~nm}$ silver NPS (bottom left), and a co-culture of $16 \mathrm{HBE}$ and THP-1 exposed to $3 \mu \mathrm{g} / \mathrm{cm}^{2}$ $20 \mathrm{~nm}$ silver NPS (bottom right). The THP-1 macrophages take up the silver NPS and thereby protect the epithelial cell layer.

\section{DISCUSSION}

In the present study, we tested silver NPs of four different sizes using in vitro models of increasing complexity to determine the most suitable dose metric in vitro. In vivo, the alveolar dose expressed as particle surface area was found to be the most suitable dose 
metric to describe the toxicity of silver NPs after inhalation (Braakhuis et al., 2015a). The results from the present study show that when using a simple monolayer of I6 $6 \mathrm{HBE}$, the particle surface area also was found to be the most suitable dose metric to describe the effect of silver NPS on metabolic activity, MCP-I and IL-8 production. When adding THP-I macrophages to the model, the dose metric shifted from particle surface area to particle mass as the number of macrophages increased. Light microscopy revealed that without macrophages, the silver NPS spread evenly over the I6HBE monolayer resulting in cellular exposure and subsequent effects. When macrophages were present on top of the I6HBE monolayer, they took up most of the silver NPs and thereby reduced the effective dose and prevented cellular exposure and effects in the epithelial cells.
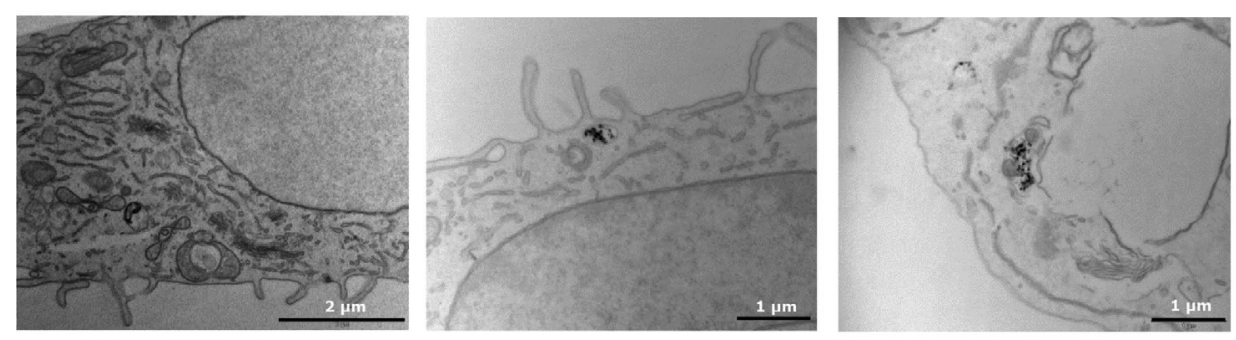

Figure 9. TEM pictures of THP-1 macrophages exposed to solvent control (left), $20 \mathrm{~nm}$ silver NPS for 6 hours (middle), and $20 \mathrm{~nm}$ silver NPS for 6 hours plus a 18 hours resting period (right). Most silver NPS are taken up after 6 hours exposure. The resting period of 18 hours did not induce visible dissolution of the $20 \mathrm{~nm}$ silver NPS.

Other in vitro studies also found that particle surface area is a suitable dose metric for silver NPs. In alveolar A 549 cells, Huk et al. showed that expressing the concentrations of silver NPS as number or in terms of surface area is more representative for evaluation of toxicity than using the conventional mass units (Huk et al., 2014). In addition, in the study of Delmaar et al., which describes the method used here to determine a suitable dose metric, particle surface area is a suitable dose metric for silver NPs to describe the metabolic activity in fibroblasts, the induction of ROs in rat macrophages, and photosynthesis in algae (Delmaar et al., 2015). However, the dose metric was different in the embryonic stem cell test, for membrane integrity in fibroblasts, immobilization in zooplankton, and teratogenicity in zebrafish (Delmaar et al., 2015, Park et al., 20I I, Wang et al., 20 I2). Similar to the results of this study, the dose metric not only depends on the characteristics of the NPs but also on the experimental design and measured endpoints. Remarkably, the simplest in vitro model of the present study most closely resembled the results of our in vivo study.

Particle surface area is not only a suitable dose metric for silver NPs, but also for NPs of other chemical compositions. For low-toxicity, low-solubility NPs like titanium dioxide, carbon black, cobalt and nickel, the suitable dose metric was particle surface area both 
in vivo (intratracheal instillation) for the influx of neutrophils and in vitro in alveolar epithelial cells for the production of IL-8 (Duffin et al., 2007). This was confirmed in another study showing that particle surface area is an appropriate dose metric for lowsolubility, low toxicity particles in alveolar epithelial cells for describing IL-8 production and glutathione depletion (Monteiller et al., 2007). Titanium dioxide NPs of different sizes were administered via intratracheal instillation in vivo and to alveolar epithelial cells in vitro. The results showed that the influx of neutrophils in vivo and the cellular damage and Ros production in vitro were well correlated when the dose was expressed as particle surface area (Han et al., 20I2). These studies confirm that when using an epithelial monolayer, the same dose metric can be found compared to inhalation or intratracheal instillation.

An explanation why particle surface area is a suitable dose metric for acute inflammation, both in vivo (Braakhuis et al., 20 I 5a) and in a monolayer of lung epithelial cells in vitro, is that silver NPS can release silver ions from their surface (Ma et al., 20 2, Leo et al., 2013, Stebounova et al., 20I Ib, Kent and Vikesland, 20I2, Zook et al., 20I I) that contribute to the observed toxicity after inhalation (Pratsinis et al., 20I3, Beer et al., 20I 2, Wang et al., 20I4b). The amount of released silver ions is related to their size and surface area (Peretyazhko et al., 20 4 , Ma et al., 20 I2). In addition, the silver NPs might cause their effects by the direct contact of their surface with the epithelial cells. Next to this, the size of silver NPs influences cellular responses, including uptake (Zhang et al., 20I4); smaller NPs enter cells more easily than larger ones, which may be another cause of stronger toxic effects (Liu et al., 20 Io). Particle surface area is a suitable dose metric for silver NPS in a monolayer of epithelial cells but not in co-cultures with different densities of macrophages. When macrophages are added to the in vitro model, the NPs are taken up making the particle volume, and thus the mass, more important. In vivo, there are normally a fixed number of macrophages in the lungs and upon an exposure macrophages are recruited. The fixed number of macrophages is very low, about one macrophage per alveolus (Gordon and Read, 2002, Kopf et al., 2015). When inhaled NPS deposit in the alveoli, they interact with the epithelial cells that are triggered to produce pro-inflammatory cytokines to recruit macrophages (Stone et al., 2007). There is thus most likely an exposure to NPs of the epithelial cells before macrophages are recruited, in vivo. This may explain why the results of the I6HBE monolayer correlate better to the in vivo data compared to the more complex models.

As explained above, particle surface area is a suitable dose metric probably because silver NPs induce effects at their surface including the release of silver ions. Dissolution of silver NPs depends on their particle size, the $\mathrm{pH}$ of the solution, the ions present in the solution (e.g. sulphate, chloride and phosphate causing precipitations with silver ions or catalysing dissolution), aggregation state and the incubation time (Ma et al., 20 I2, Leo et al., 20I3, Stebounova et al., 20I Ib, Kent and Vikesland, 20I2, Zook et al., 20II). In our study, there was no silver ion release from the particles in culture medium up to 24 hours. In addition, there was no silver ion release in artificial lysosomal fluid (ALF) of 
$\mathrm{pH} 4.6$, while there was complete dissolution of the particles in $\mathrm{HNO}_{3}$ of $\mathrm{pH} 4.6$ after 5 hours incubation. There are many chlorides and phosphates present in ALF which probably caused precipitation of the released silver ions resulting in an absence of free silver ions that could be detected by the ion selective electrode, although the silver ions probably were released in ALF. In addition, the TEM images did not show any intracellular dissolution of $20 \mathrm{~nm}$ silver NPS after 6 hours exposure and 18 hours post-incubation. The TEM images reveal that the NPs are taken up in cytoplasmic vesicles but we did not observe silver NPs in lysosomes nor fusion of the vesicles with lysosomes, explaining why we did not observe intracellular dissolution. Possibly, the fusion with lysosomes requires more time. Once in the lysosomes, the silver NPs most likely do release silver ions.

The present in vitro study focused on acute effects of nanomaterials. Chronic effects of nanomaterials and the effects after long-term or repeated exposure are probably different from the observed acute epithelial damage. After long-term or repeated exposure, clearance mechanisms and secondary effects play a more important role. Therefore, a simple monolayer of epithelial cells will probably not be sufficient to determine the effects of nanomaterials after repeated or long-term exposure and more complex in vitro models might be needed.

\section{CONCLUSION}

This study shows that when using a simple monolayer of lung epithelial cells, the most suitable dose metric to describe the effect of silver NPs is their particle surface area, similar to the dose metric found in vivo. Increasing the complexity of the in vitro model by adding alveolar macrophages and culture the model on Transwell ${ }^{\circ}$ inserts results in less correlation with in vivo data. We conclude that for studying the acute pulmonary toxicity of NPs, simple in vitro models using an epithelial monolayer better predict the in vivo inflammatory response compared to complex co-culture models.

\section{ACKNOWLEDGEMENTS}

This work was supported by the project 'Integrated Risk Assessment of Nanomaterials' from the National Institute for Public Health and the Environment and by the NanoNextnl program 'Risk Analysis and Technology Assessment: Human Health Risks'. We would like to thank Adrienne J.A.M. Sips, Agnes G. Oomen and Rob Vandebriel for critical review of the manuscript. 
Hedwig M. Braakhuis ${ }^{\mathrm{I}, 2^{*}}$, Marco Dilger ${ }^{3}$, Sonja Mülhopt ${ }^{4}$, Margriet V. D. Z. Park ${ }^{2}$, HannsRudolf-Paur ${ }^{4}$, Carsten Weiss ${ }^{3}$, Silvia Diabaté ${ }^{3}$

${ }^{\mathrm{I}}$ Department of Toxicogenomics, Maastricht University, po Box 6I 6, 6200 MD Maastricht, the Netherlands

${ }^{2}$ National Institute for Public Health and the Environment (RIVM), Po Box I, 3720 BA Bilthoven, the Netherlands

${ }^{3}$ Institute of Toxicology and Genetics, Karlsruhe Institute of Technology, Campus North, Hermann-von-Helmholtz-Platz I, 76344 Eggenstein-Leopoldshafen, Germany

${ }^{4}$ Institute for Technical Chemistry, Karlsruhe Institute of Technology, Campus North,

Hermann-von-Helmholtz-Platz I, 76344 Eggenstein-Leopoldshafen, Germany 
Exposure of an in vitro lung model to silver nanoparticles at the air-liquid interface 


\section{ABSTRACT}

To reduce animal testing, several in vitro lung models have been developed to test the effect of nanomaterials upon exposure to an aerosol. Air-liquid models have the advantage that nanoparticles are not altered during exposure by contact with culture medium and the exposure doses and dose rate are more similar to in vivo inhalation compared to submerged exposure conditions. The aim of this preliminary study was to determine whether effectdoses after exposure to nanoparticles at the air-liquid interface correlates better to the in vivo situation compared to submerged exposure. We exposed an in vitro lung model to silver NPS of different sizes at the air-liquid interface using a well-characterized exposure chamber system. In addition, we challenged the lung model with NPs with and without the presence of alveolar macrophages. Cell viability and the release of pro-inflammatory cytokines were measured as an indicator of pulmonary toxicity. In the lung model without macrophages, exposure to $15 \mathrm{~nm}$ and $30 \mathrm{~nm}$ silver NPs at the air-liquid interface caused a dose-dependent decrease in cell viability and increase in MCP-I and IL-8 similar to in vivo. Addition of macrophages resulted in a reduction of the effective dose and a protection of the epithelial cells. The doses at which significant changes in pulmonary toxicity parameters were observed after air-liquid exposure $\left(0.0025-0.02 \mu \mathrm{g} / \mathrm{cm}^{2}\right)$ were similar to the in vivo deposited doses $\left(0.000 \mathrm{I}-0.005 \mu \mathrm{g} / \mathrm{cm}^{2}\right)$ while under submerged conditions extremely high doses were needed to observe significant effects $(3-30 \mu \mathrm{g} /$ $\mathrm{cm}^{2}$ ). The air-liquid exposures should be repeated in the future to confirm that they better mimic the exposure and effects that occur in vivo compared to submerged exposure.

\section{INTRODUCTION}

Inhalation is considered an important exposure route for nanomaterials. To reduce toxicity testing using animal studies, several in vitro lung models have been developed; varying from a single cell type to the co-culture of different cell types (Brakhuis et al., 20 I 5c). Besides differences in cell types, in vitro lung barrier models differ in whether they are submerged or cultured at the air-liquid interface. Submerged models have the advantage of being technically simple. However, in vitro exposure of lung cells under submerged conditions does not reflect the physiological situation in the lung where cells are directly exposed to an aerosol (Panas et al., 20 I 4). Additionally, the culture medium can alter the physicochemical properties of the nanoparticles (NPS), and subsequently their uptake and effects. Finally, the delivered dose in culture models under submerged conditions is often unclear due to differences in agglomeration and sedimentation of suspended NPs (Panas et al., 20I4). Air-liquid models mimic more realistically the inhalation exposure and use exposure doses and dose rates that are more similar to the in vivo situation. For this reason, various air-liquid models have been developed recently (Blank et al., 2006, Brandenberger et al., 20Iob, Frohlich et al., 2013, Herzog et al., 2013, Holder and Marr, 2013, Lenz et al., 2013, Lenz et al., 2009, Raemy et al., 20I I, Rothen-Rutishauser et al., 2009, Savi et al., 2008, Xie et al., 20 I 2, Diabate et al., 2008). 
These air-liquid models can be used for testing NPs as an aerosol generated from powders or liquids.

The in vitro lung model used in this study consists of a co-culture of human bronchial epithelial cells ( $\mathrm{I} 6 \mathrm{HBE})$ and differentiated human alveolar macrophages (THP-I) at the apical side and human umbilical vein endothelial cells (HUVEC) at the basolateral side of a Transwell ${ }^{\circ}$ insert membrane (figure I). This model was published recently by Luyts et al. (Luyts et al., 2015). The I6HBE cells are ciliated and form tight junctions which are important for optimal barrier function (Zhu et al., I999, Lehmann et al., 20I I, Chowdhury et al., 2010). Together with the endothelial cells, they form the lung-blood barrier. Alveolar macrophages were added to this lung model because they are known to protect the epithelial cells from damage induced by NPs via phagocytosis and the release of mediators. This lung model was exposed to silver NPs of different sizes under submerged conditions previously (Braakhuis et al., 2015b). The results show that for studying the dose metric of acute pulmonary toxicity of silver NPs, simple submerged in vitro models using an epithelial monolayer better predict the in vivo response compared to complex co-culture models (Braakhuis et al., 2015b). Although the dose metric for silver NPs in both the in vivo study and the epithelial monolayer was the same, the dose that was needed to find a significant effect was a factor of 6 to 6000 times higher in the submerged in vitro model compared to the in vivo model, probably as a result of the difference in dose rate and dissolution. We therefore hypothesize that air-liquid exposure using the lung barrier model will overcome these differences. In the study presented here, we have used silver NPs of different sizes to determine their toxicity and we correlated the results with those from the submerged exposure as well as our previous in vivo work (Braakhuis et al., 201 5a). In addition we explored the impact of the presence of macrophages in the lung barrier model. Our hypothesis is that effectdoses after air-liquid exposure of the in vitro lung model better predict the in vivo effectdoses compared to submerged exposure.

A

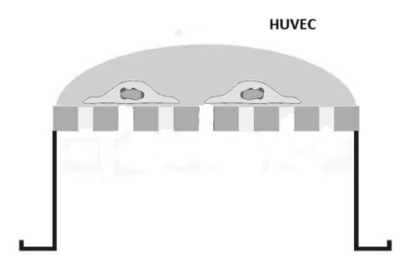

B

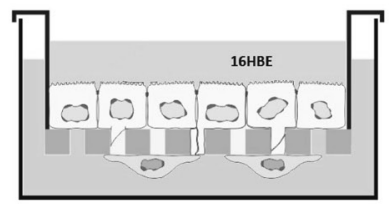

C

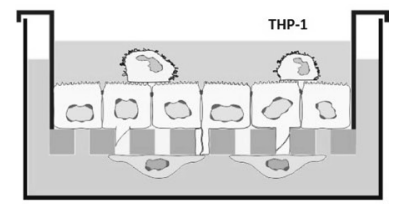

Figure 1. Schematic representation of the lung barrier model. HuvEc cells were seeded at the basolateral side of the Transwell ${ }^{\circledR}$ insert membrane (A), after 2-3 hours 16 HBE cells were seeded at the apical side of the insert membrane (B), finally differentiated THP-1 cells were added to the apical side of the bi-culture (C). Figure adapted from Rothen-Rutishauser et al. 2005 (Rothen-Rutishauser et al., 2005). 
The QualityNano (QNano) Transnational Access program of the QNano Research Infrastructure sponsored the air-liquid exposures by enabling cooperation between the National Institute of Public Health of the Netherlands and the Karlsruhe Institute of Technology (КIT). At the KIT, we used a well-characterized exposure chamber system (VITROCEll ${ }^{\circ}$ SYSTEMs) that is previously described in detail (Comouth et al., 20I3, Panas et al., 20I 4, Diabate et al., 2008, Mülhopt et al., 2009) to expose the lung barrier model to silver NPs. In total, three visits of each two weeks were sponsored by the QNano Transnational Access program in 2013, 2014 and 2015. The results of these visits are presented in this chapter.

\section{MATERIALS AND METHODS}

\section{Nanomaterials}

The test atmosphere was produced by mixing silver NPS with HEPA filtered and conditioned $\left(\mathrm{I} 3 \% \mathrm{RH}, 2 \mathrm{I}^{\circ} \mathrm{C}\right)$ compressed air. The silver NPs were produced by a Palas GFG Iooo (Palas GmbH, Karlsruhe, Germany) spark generator fitted with silver tipped electrodes. To generate the smallest aerosol size ( $15 \mathrm{~nm} \mathrm{CMD)}$ ), the output of the generator was immediately diluted in conditioned air. To generate the two intermediate aerosol sizes of $30 \mathrm{~nm}$ and $45 \mathrm{~nm}$, a longer residence time of spark generated particles in mixing chambers was used to lead the aerosol into the exposure chamber. This increased residence time resulted in aggregation, which is a function of time. In order to obtain spherical particles, the aerosol was heated by passing it via tubing through an oven at $600{ }^{\circ} \mathrm{C}$, which resulted in fusing of aggregates. The particle number concentration was controlled by the spark generator frequency.

\section{Test atmosphere characterization}

The particle number concentration was measured by a condensation particle counter CPC 3775 (TSI inc., St Paul MN, USA). Particle size distribution was monitored by a scanning mobility particle sizer (sMPs) 3775 with 307 I differential mobility analyser (DMA) (TSI inc., St Paul MN, USA). The particle mass concentration was calculated with the density of silver $\left(\mathrm{I} 0.49 \mathrm{~g} / \mathrm{cm}^{3}\right)$ and the particle number distribution measured by SMPS.

In order to determine the deposited fraction of the applied aerosol on the cells during ALI exposure, transmission electron microscopy (TEM) grids (Plano, SFi62-6) were placed on the Transwell ${ }^{\bullet}$ membranes at three different radial positions and exposed to the aerosol for different times. Subsequently, 50 micrographs of the deposited particles were taken for each experiment using a TEM (Zeiss I o9T, Oberkochen). The deposited particles were detected and analysed regarding their number and size with custommade software (Comouth et al., 2013). For the calculation of mass doses, we used the density of silver of $10.49 \mathrm{~g} / \mathrm{cm}^{3}$. The total amount of deposited particles was estimated 
by extrapolating the amount of deposited particles as a function of the applied particle mass.

\section{Cells and cell culture conditions}

Human bronchial epithelial cells I6HBEI4O- cells (I6HBE) were kindly provided by Dr. Gruenert (University of California, San Francisco, USA) and cultured in DMEM/FI 2 medium supplemented with $5 \%$ FCS, I $00 \mathrm{U} / \mathrm{ml}$ penicillin, Ioo $\mu \mathrm{g} / \mathrm{ml}$ streptomycin, $2 \mathrm{mM} \mathrm{L}$-glutamine and $2.5 \mu \mathrm{g} / \mathrm{ml}$ fungizone. Human umbilical vein endothelial cells (HUVEC) were purchased from Life Technologies (Thermo Fisher Scientific Inc., the Netherlands) and cultured in M200 supplemented with LSGS, IOO U/ml penicillin, IOO $\mu \mathrm{g} / \mathrm{ml}$ streptomycin and $0.5 \mu \mathrm{g} / \mathrm{ml}$ fungizone. Human acute monocytic leukemia cells (THP-I) were purchased from American Type Culture Collection (ATCC, Rockville, USA) and cultured in RPMI medium supplemented with Iо\% FCS, IO० U/ml penicillin, and IоO $\mu \mathrm{g} / \mathrm{ml}$ streptomycin. To differentiate the THP-I monocytes into macrophages, the cells were seeded at a density of 500000 cell $/ \mathrm{ml}$ in the presence of $30 \mathrm{ng} / \mathrm{ml}$ PMA and were further incubated for 5 days. At day 5 , the medium was removed and replaced by full culture medium and cells were cultured for 3 days.

The cells were incubated at $37{ }^{\circ} \mathrm{C}$ in a $100 \%$ humidified atmosphere containing $5 \%$ $\mathrm{CO}_{2}$. When confluent, the epithelial and endothelial cells were released enzymatically (0.05\% trypsin-EDTA for I6HBE and HUVEC, Accutase for differentiated THP-I) and transferred to a new culture flask. The medium was renewed every 2-3 days.

\section{Preparation of the lung model}

The lung model was prepared on non-coated polyester Transwell ${ }^{\circ}$ insert membranes with $0.4 \mathrm{um}$ pores and a surface area of $4.67 \mathrm{~cm}^{2}$ (inserts fit in a 6-wells plate, compatible with the ALI system). For the bi-culture model, HUVEC cells were seeded in a density of 50000 cells $/ \mathrm{cm}^{2}$ on the basolateral side of the transwell inserts. After 2 hours, the Transwell ${ }^{\odot}$ inserts were returned to their original orientation and $16 \mathrm{HBE}$ cells were seeded in a density of 100 ooo cells $/ \mathrm{cm}^{2}$ on the apical side. For the tri-culture model, 4 hours after seeding of the I6HBE cells, THP-I macrophages were added to the bi-culture model at the apical side in a ratio of I: IO (I THP-I cell per Io I6HBE cells).

\section{Exposure}

For the air-liquid exposures, the Karlsruhe Exposure System was used (figure 2). The whole setup consists of two units: a data acquisition and control unit as well as a cell exposure unit. The exposure unit consists of a humidification unit, exposure modules and a characterization unit. The air containing the silver NPS was first humidified to $85 \%(+/-\mathrm{I} \%)$ relative humidity to ensure cell survival. After humidification, a sample flow for each position is isokineticly taken out of the aerosol reactor. This flow is lead to 
the VITROCELL ${ }^{\oplus} 6 / 3$ exposure modules in which cells are located. The aerosol is guided through the aerosol inlet towards the cell surface where the aerosol gets in direct contact at the air liquid interface with cells. There are four exposure modules for a total of twelve $24 \mathrm{~mm}$ Transwell ${ }^{\circ}$ inserts. To enhance deposition, three exposure chambers were equipped with electrodes that applied an electrostatic potential between the cells and the aerosol inlet $(-\mathrm{IOOOOV})$. The modules were temperature controlled with water at $37.5^{\circ} \mathrm{C}$. In combination with the incubator and humidifier a stable humidity and temperature within the exposure chambers was ensured. The aerosol flow through each tube was set by mass flow controllers (Bronkhorst, EL-Flow) to Ioo $\mathrm{ml} / \mathrm{min}$.

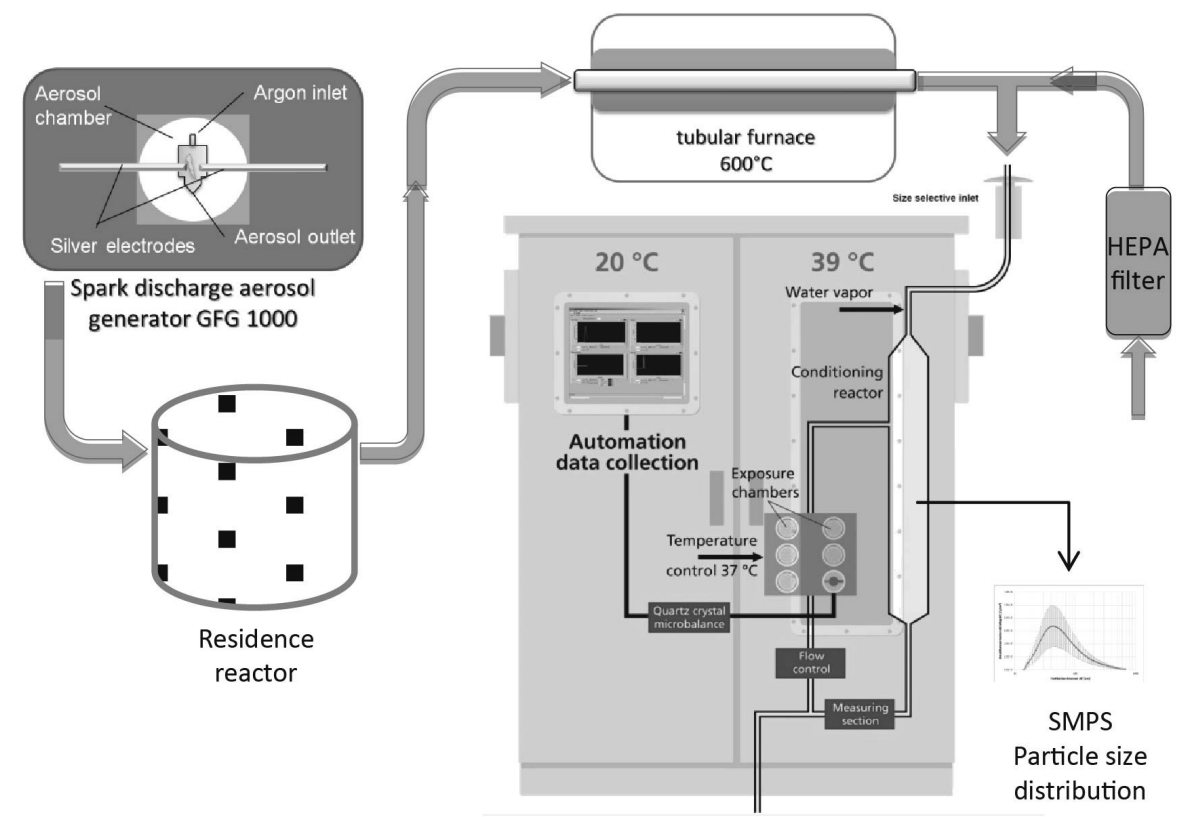

Figure 2. Setup for the air-liquid exposures. Silver nanoparticles generated by spark discharge pass a residence reactor followed by a tubular furnace. There the agglomerates sinter to bigger particles which were led to the Karlsruhe Exposure System equipped with human cell lines.

Prior to exposure, the culture medium at the apical side of the Transwell ${ }^{\circ}$ inserts was removed and the culture medium at the basolateral side was replaced by RPMI I 640 medium containing $25 \mathrm{mM}$ HEPES, supplemented with $100 \mathrm{U} / \mathrm{ml}$ penicillin and IOO $\mu \mathrm{g} / \mathrm{ml}$ streptomycin. The inserts were placed in the exposure system and exposed for 4 hours at the air-liquid interface. Three inserts with cells were exposed to clean air. Three other inserts were exposed to the filtered aerosol which was generated by passing the humidified and conditioned aerosol through HePA air filters (HepaVent ${ }^{\mathrm{TM}}$ Y27I, , Whatman GmbH, Dassel). Finally, six inserts were exposed to the unfiltered aerosol 
of which three without electrostatic deposition and three with electrostatic deposition $(-\mathrm{r} 000 \mathrm{~V})$. After 4 hours exposure, the Transwell ${ }^{\circledR}$ inserts were taken out of the air-liquid exposure unit, culture medium was applied to both the apical and basolateral side of the inserts and they were incubated at $37^{\circ} \mathrm{C}$ in a $100 \%$ humidified atmosphere containing $5 \% \mathrm{CO}_{2}$ for 20 hours.

\section{Membrane integrity}

Cell membrane integrity of the lung model after air-liquid exposure was evaluated by determining the activity of lactate dehydrogenase (LDH) leaking out of the cells, using the Cytotoxicity Detection Kit (LDH) (Roche Diagnostics GmbH, Mannheim, Germany) according to manufacturer's specifications. The lung model was exposed to silver NPs via the air-liquid interface for 4 hours. Ioo $\mu \mathrm{l}$ of the medium at the basolateral side of the inserts was used in the LDH assay to determine lactate dehydrogenase (LDH) release following membrane disruption. Extracellular LDH was corrected for total LDH in cells (measured in medium of lung models lysed using Triton X-гоo), to avoid false negative results in cases where the nanoparticle treatment caused an inhibition of cell proliferation and as such caused a lower amount of LDH to be available to leak out of damaged cells.

\section{Metabolic activity}

The viability of the lung model was determined using the CellTiter $96^{\circ}$ AQueous One Solution Cell Proliferation Assay (MTS, Promega, G3582) according to manufacturer's specifications. The reagent contains a tetrazolium compound: 3-(4,5-dimethylthiazol2-yl)-5-(3-carboxymethoxyphenyl)-2-(4-sulfophenyl)-2H-tetrazolium, inner salt; MTs. Briefly, MTs is reduced by metabolically active cells into a coloured formazan product that is soluble in tissue culture medium. The quantity of formazan product as measured by the absorbance at $490 \mathrm{~nm}$ is directly proportional to the bulk metabolic activity of cells in culture. After air-liquid exposure, culture medium was applied to both the apical and basolateral side of the Transwell ${ }^{\odot}$ inserts. After 20 hours incubation at $37^{\circ} \mathrm{C}$, the culture medium was removed and the MTS reagent was applied to the apical side for I hour and transferred to a 96-wells plate to measure the absorbance at $490 \mathrm{~nm}$.

\section{Inflammation markers}

Release of inflammation markers MCP-I and IL- 8 were measured in the culture medium at the basolateral side of the lung models directly after exposure to silver NPs and in the culture medium at the apical and basolateral side of the lung models at 20 hours after the end of the exposure using ELISA (eBioscience, Inc.). Cytokine analysis was performed according to the manufacturer's instructions. Absorbance was measured using a SpectraMax I90 scanning multiwell spectrophotometer (Molecular Devices, Sunnyvale, USA) at a wavelength of $450 \mathrm{~nm}$ and a reference wavelength of $570 \mathrm{~nm}$. 


\section{Statistical analysis}

During each visit in 2013, 20I4 and 2015, different exposure concentrations were generated that resulted in different estimated deposited doses (table I). By combining the data, we were able to produce dose-response figures for the LDH release, MTS reduction, MCP-I production and IL- 8 production. The data was analysed by the Kruskal Wallis nonparametric test (Graphpad Prism). Statistical significance is indicated with a $*$ ( $p=$ $<0.05)$. The data presented here is based on single experiments; the replicates represent different Transwell ${ }^{\circ}$ inserts that are exposed within the same experiment. In all graphs, error bars represent the standard deviation of the mean.

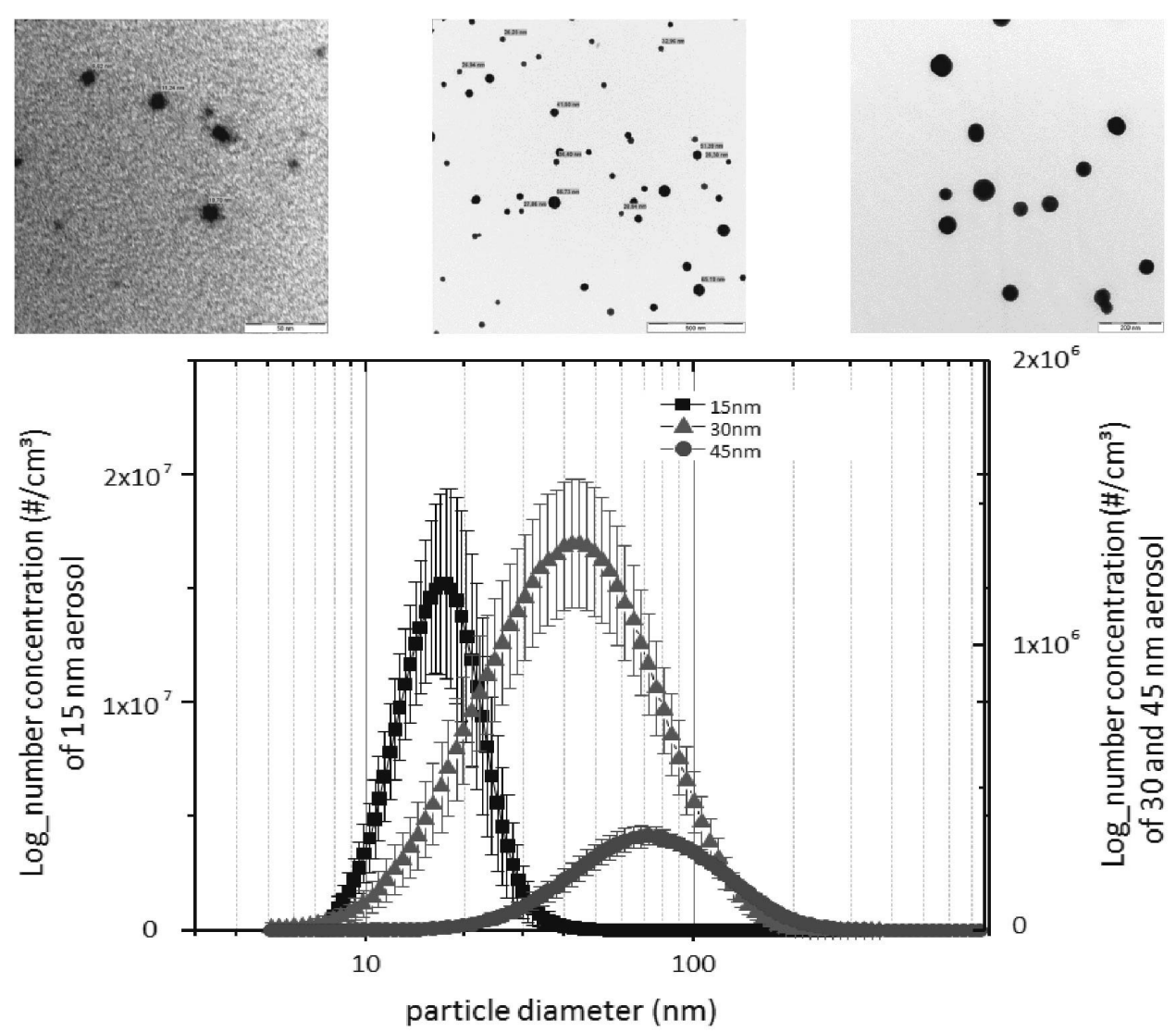

Figure 3. Particle size distribution and number of the silver NPS generated by Palas spark generation, measured by SMPS (bottom) and confirmed by TEM (top). 


\section{RESULTS}

\section{Test atmosphere characterization}

The nanoparticle characteristics are summarized in table $\mathrm{I}$. The deposited dose was estimated from the TEM pictures as described above. For all experiments, the estimated dose was about 8 -fold higher in the lung models exposed to the aerosols with application of high voltage compared to the lung models exposed without high voltage. Different deposited mass doses were estimated for the three particle sizes during the different visits. The estimated deposited mass doses on the cells ranged from 0.0025 to $0.12 \mu \mathrm{g} /$ $\mathrm{cm}^{2}$ for the $15 \mathrm{~nm}$ NPs, from 0.0027 to $0.093 \mu \mathrm{g} / \mathrm{cm}^{2}$ for the $30 \mathrm{~nm} \mathrm{NPs}$, and from 0.0053 to $0.07 \mu \mathrm{g} / \mathrm{cm}^{2}$ for the $45 \mathrm{~nm}$ NPs. The size distributions of the silver NPs are measured by SMPS and confirmed by TEM (figure 3 ).

\section{Membrane integrity}

The membrane integrity was measured by quantifying the amount of LDH released in the medium at the basolateral side of the Transwell ${ }^{\circ}$ insert after 4 hours exposure at the air-liquid interface (figure 4). Exposure to the silver NPs slightly increased LDH release by the cells. In comparison to the lysed cells, the LDH release in the exposed cells was about Io fold lower (data not shown). The data show a dose-dependent increase in LDH release after exposure to $15 \mathrm{~nm}$ silver NPs in the bi-culture model and after exposure to all particle sizes in the tri-culture model. LDH release was significantly increased compared to the clean air controls after exposures to mass doses of $0.02 \mu \mathrm{g} / \mathrm{cm}^{2}$ and above.
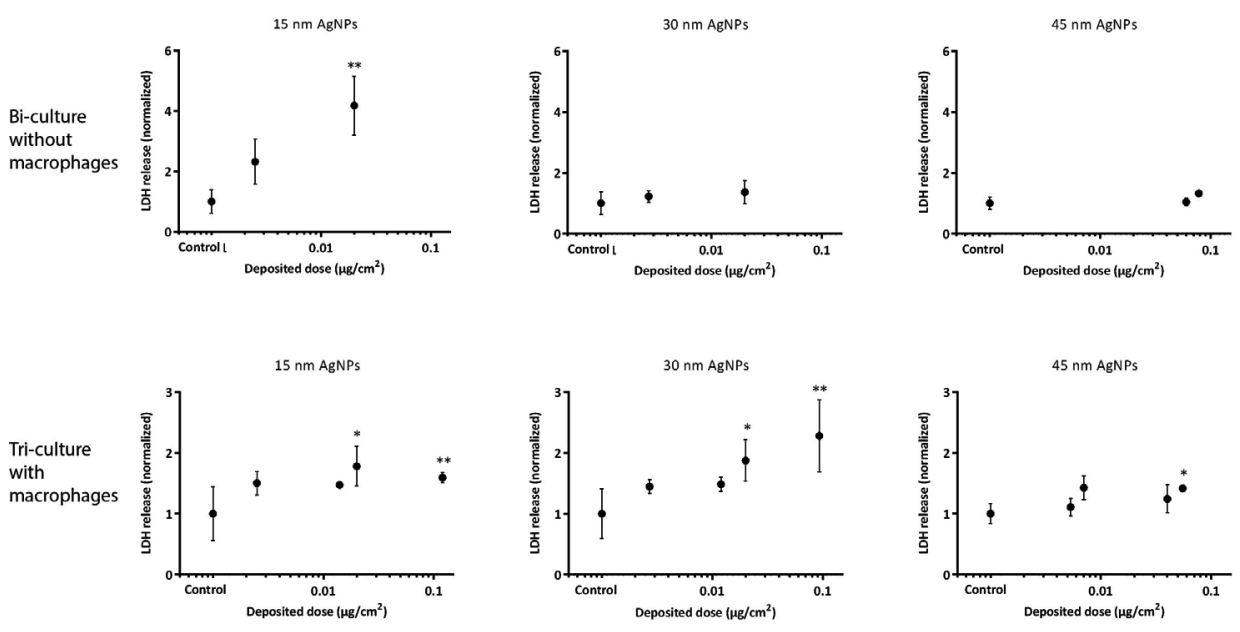

Figure 4. LDH release in bi-culture and tri-culture long models exposed to $15 \mathrm{~nm}, 30 \mathrm{~nm}$ and $45 \mathrm{~nm}$ silver nanoparticles at the air-liquid interphase. $\left({ }^{*}=p<0.05,{ }^{* *}=p<0.01\right)$. Data from single experiments; the replicates represent different Transwell ${ }^{\circledR}$ inserts that are exposed within the same experiment (mean with SD). 


\section{Metabolic activity}

Metabolic activity was measured by quantifying the mTs reduction in the apical medium of the Transwell ${ }^{\circ}$ inserts at 20 hours post-incubation (figure 5). Exposure to the silver NPS resulted in a decrease in MTS conversion, indicating decreased cell viability. The MTS conversion decreased significantly after exposure to $0.0025 \mu \mathrm{g} / \mathrm{cm}^{2}$ of I $5 \mathrm{~nm}(p=0.038)$ and $0.02 \mu \mathrm{g} / \mathrm{cm}^{2}$ of $30 \mathrm{~nm}$ silver NPs $(p=0.0024)$ in the bi-culture model and 0.007 and $0.055 \mu \mathrm{g} / \mathrm{cm}^{2}$ of $45 \mathrm{~nm}$ silver NPs in the tri-culture model ( $p=0.0 \mathrm{I}$ and $p=0.0039$, respectively).
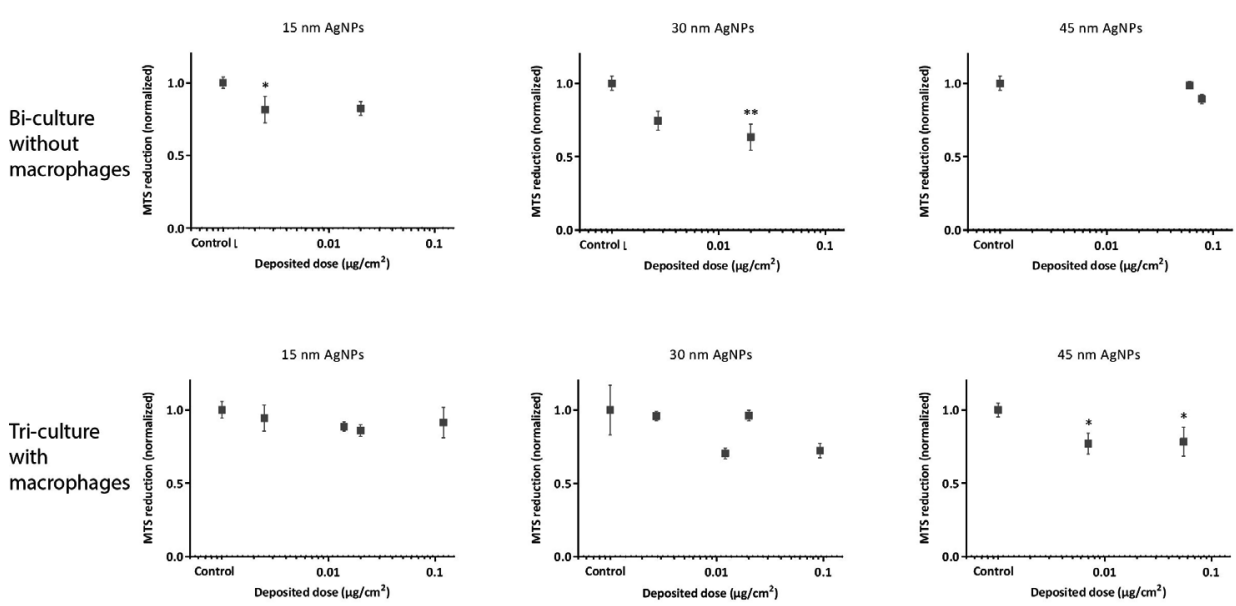

Figure 5. MTS reduction in bi-culture and tri-culture long models exposed to $15 \mathrm{~nm}, 30$ $\mathrm{nm}$ and $45 \mathrm{~nm}$ silver nanoparticles at the air-liquid interphase measured in the apical medium at 20 hours after the end of the exposure. $\left({ }^{*}=p<0.05,{ }^{* *}=p<0.01\right)$. Data from single experiments; the replicates represent different Transwell ${ }^{\circledR}$ inserts that are exposed within the same experiment (mean with SD).

\section{Inflammation markers}

The production of MCP-I and IL-8 was measured in the culture medium I) at the basolateral side of the inserts directly after the air-liquid exposure to silver NPs, 2) at the apical side of the inserts 20 hours after exposure, and 3) at the basolateral side of the inserts 20 hours after exposure. In the medium at the basolateral side directly after exposure, there were no changes in MCP-I production between the different exposure groups in both the bi-culture tri-culture model as the production and release of cytokines takes time (data not shown). In the medium at the apical side collected 20 hours after the exposure, there was a dose-dependent increase in MCP-I production in the bi-culture model after air-liquid exposure to $15 \mathrm{~nm}$ and $30 \mathrm{~nm}$ silver NPs. The MCP-I production was significantly increased after exposure to $0.02 \mu \mathrm{g} / \mathrm{cm}^{2}$ of I $5 \mathrm{~nm}$ and $30 \mathrm{~nm}$ silver NPS 
in the bi-culture model ( $p=0.04 \mathrm{I} 5$ and $p=0.050$ ) (figure 6 ). In the tri-culture model with macrophages, there were no significant changes in MCP-I production.

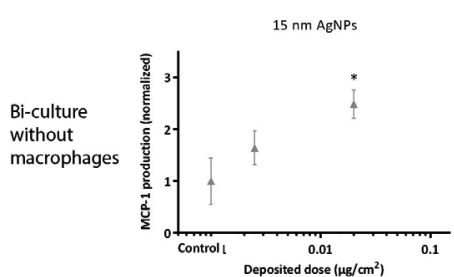

$15 \mathrm{~nm}$ AgNPs

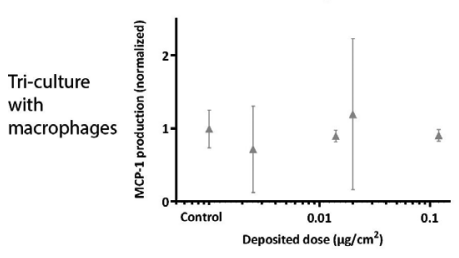

$30 \mathrm{~nm}$ AgNPs

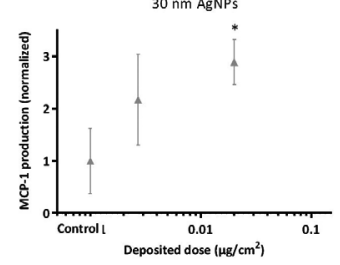

$30 \mathrm{~nm}$ AgNPS

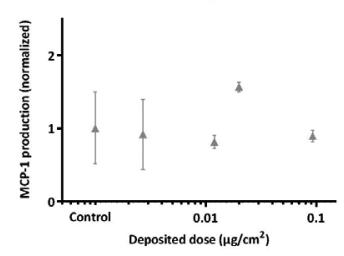

$45 \mathrm{~nm}$ AgNPs

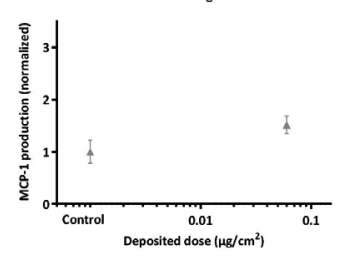

$45 \mathrm{~nm}$ AgNPs

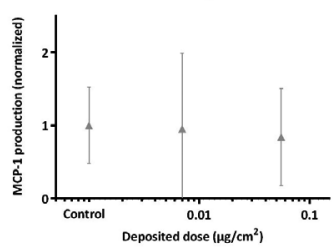

Figure 6. MCP-1 production in medium at the apical side of bi-culture and tri-culture long models 20 hours after exposure to $15 \mathrm{~nm}, 30 \mathrm{~nm}$ and $45 \mathrm{~nm}$ silver nanoparticles at the air-liquid interphase. $\left({ }^{*}=p<0.05\right)$. Data from single experiments; the replicates represent different Transwell ${ }^{\circledR}$ inserts that are exposed within the same experiment (mean with sD).
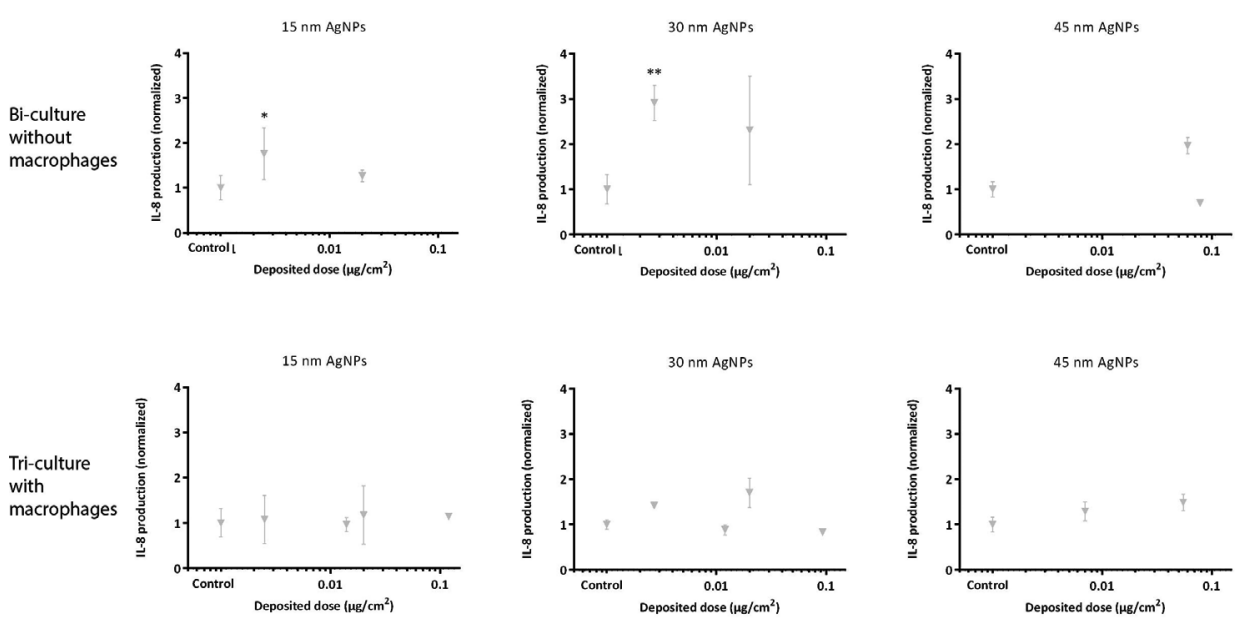

Figure 7. $\mathrm{LL}-8$ production in medium at the apical side of bi-culture and tri-culture long models 20 hours after exposure to $15 \mathrm{~nm}, 30 \mathrm{~nm}$ and $45 \mathrm{~nm}$ silver nanoparticles at the air-liquid interphase. $\left({ }^{*}=p<0.05,{ }^{* *}=p<0.01\right)$. Data from single experiments; the replicates represent different Transwell ${ }^{\circledR}$ inserts that are exposed within the same experiment (mean with SD). 
There were no changes in IL- 8 production between the different groups in the bi-culture model measured in the medium at the basolateral side directly after exposure (data not shown). In the tri-culture model, air-liquid exposure to $0.012 \mu \mathrm{g} / \mathrm{cm}^{2}$ of the $30 \mathrm{~nm}$ silver NPs induced a significant increase in IL-8 production (data not shown). In the medium at the apical side collected 20 hours after the exposure, there was a significant increase in IL-8 production after air-liquid exposure to $0.0025 \mu \mathrm{g} / \mathrm{cm}^{2}$ of I $5 \mathrm{~nm}$ and $0.0027 \mu \mathrm{g} / \mathrm{cm}^{2}$ of $30 \mathrm{~nm}$ silver NPs in the bi-culture model $(p=0.0303$ and $p=0.0022)$ (figure 7 ). There were no significant changes in IL-8 production in the tri-culture model at 20 hours after exposure.

\section{DISCUSSION}

The aim of this chapter is to determine I) whether effect-doses after exposure of a lung model to silver NPs at the air-liquid interface correlate better to the in vivo situation compared to previously tested submerged exposure (Braakhuis et al., 2015b), and 2) whether the addition of alveolar macrophages to the lung model improves the correlation with in vivo effect-doses. Before we compare the effect-doses between the studies, we compare the deposited doses. In the present study, the estimated deposited doses after air-liquid exposure range from 0.0025 to $0.12 \mu \mathrm{g} / \mathrm{cm}^{2}$. In our previous in vivo inhalation study, we estimated the deposited alveolar dose using the measured internal lung dose in combination with the multiple path particle dosimetry (MPPD) model to estimate the mass of silver NPs that deposited in the head, tracheobronchial and alveolar region of the lungs (Braakhuis et al., 20I 5a). Exposure to 43 - I I09 $\mu \mathrm{g}$ silver NPs per $\mathrm{m}^{3}$ air via inhalation in vivo resulted in a calculated deposited dose in the alveoli that ranged from $0.000 \mathrm{I}$ to $0.005 \mu \mathrm{g} / \mathrm{cm}^{2}$ (Brakkhuis et al., 201 5a). The lower doses in the present air-liquid experiments are similar to the doses that reached the alveoli in vivo. In the submerged exposures in vitro, the applied concentration was much higher compared to in vivo and ranged from 0.03 to $30 \mu \mathrm{g} / \mathrm{cm}^{2}$ (Brakhuis et al., 201 $5 \mathrm{~b}$ ). Using the in vitro sedimentation diffusion dosimetry (ISDD) model (DeLoid et al., 20I4), we could estimate the deposited dose in the submerged system. For the highest concentrations of Io and $30 \mu \mathrm{g} / \mathrm{cm}^{2}$, the deposited fraction was I, showing that all silver NPs deposited on the cells. When we look at the responses, for example the cell viability and MCPI production, we observe a large difference in the doses that are needed to induce a significant effect. The lowest doses at which a significant decrease in cell viability and a significant increase in MCP-I was observed are $0.002 \mu \mathrm{g} / \mathrm{cm}^{2}$ in vivo (Braakhuis et al., 20 I $5 \mathrm{a}$ ), $0.02 \mu \mathrm{g} / \mathrm{cm}^{2}$ after air-liquid exposure and Io $\mu \mathrm{g} / \mathrm{cm}^{2}$ after submerged exposure (Braakhuis et al., 20 I 5b). This shows that using air-liquid exposure, effects are induced at doses that are close to the doses used in in vivo studies and shows the extreme doses that are needed after submerged exposure to induce an effect.

The differences in exposure doses between in vivo studies, air-liquid experiments and experiments under submerged conditions can also be seen in previously published 
studies. Previous in vivo inhalation studies with silver NPs used exposure concentrations of 0.48 to a maximum of $3300 \mu \mathrm{g}$ silver NPs per $\mathrm{m}^{3}$ air (Sung et al., $20 \mathrm{I} \mathrm{Ib}$, Ji et al., 2007, Stebounova et al., 201 Ia, Song et al., 20 I 2, Sung et al., 2008, Sung et al., 2009). Although the exposure concentrations in air were similar between these studies and our previous inhalation study, in which the exposure concentration ranged from 43 to I I09 $\mu \mathrm{g}$ silver NPS per $\mathrm{m}^{3}$ air (Braakhuis et al., 201 $5 \mathrm{a}$ ), most of these studies did not estimate the alveolar dose. Previously reported studies using air-liquid exposure show deposited doses of 0.03 and $0.278 \mu \mathrm{g} / \mathrm{cm}^{2}$ (Herzog et al., 20I3) and $0.7 \mu \mathrm{g} / \mathrm{cm}^{2}$ silver NPs (Holder and Marr, 2013). These doses are somewhat higher compared to the deposited doses in the present study between 0.0025 and $0.12 \mu \mathrm{g} / \mathrm{cm}^{2}$. The submerged exposures in vitro ranged from 0.03 to $30 \mu \mathrm{g} / \mathrm{cm}^{2}$ (Braakhuis et al., 20 I $5 \mathrm{~b}$ ). These doses seem high in comparison with the in vivo studies and the air-liquid exposures; however, they are commonly used in in vitro studies with silver NPs (Park et al., 20 I , Gliga et al., 20 I 4, Carlson et al., 2008, Nymark et al., 2013). Overall, the doses tested in vitro after submerged exposure were all a factor of at least 6 ranging to 6000 higher compared to the highest dose tested in vivo, while the deposited doses after air-liquid exposure were more in line with the in vivo exposures. Exposure modelling suggests that when using silver NPS containing products like a throat spray, up to $70 \mathrm{ng}$ of silver may deposit in the respiratory tract per use (Quadros and Marr, 20II). When assuming an human alveolar surface area of $45 \mathrm{~m}^{2}$ (Hasleton, 1972), the modelled exposure is $0.000 \mathrm{I} 55 \mathrm{ng} /$ $\mathrm{cm}^{2}$ per use of the throat spray. The modelled worst case exposure is similar to the in vivo and air-liquid exposure, but orders of magnitude lower compared to the in vitro exposure under submerged conditions.

Besides effect-doses that are more similar between air-liquid exposure and in vivo exposure, air-liquid exposure has more advantages compared to submerged exposure. Using air-liquid exposure, the NPs are not altered by contact with culture medium. It is known that for example in culture medium with serum, proteins and other medium components form a corona around silver NPs and thereby modify their toxicity (Shannahan et al., 2015). In addition, NPs tend to agglomerate in medium (Mukherjee et al., 2014), which potentially influences their uptake and effect. Furthermore, in airliquid exposure a constant air-flow containing NPs is generated and directed to the cells similar to inhalation of NPS, while under submerged conditions the NPs are applied all at once. Disadvantages are that there are only few air-liquid exposure systems available and their development is expensive. In addition, the exposure conditions like humidity, temperature, and particle concentration can vary more in an air-liquid exposure system compared to submerged exposure.

Next, we were interested whether the addition of alveolar macrophages improves the lung model. Exposure of a submerged lung model to silver NPs showed that a monolayer of lung epithelial cells correlates better to in vivo data compared to a coculture model including alveolar macrophages (Braakhuis et al., 2015b). The alveolar macrophages phagocytize the silver NPS and thereby reduce the effective dose and 
protect the epithelial layer from damage. Exposure at the air-liquid interface shows the same results. In the bi-culture model without macrophages, $15 \mathrm{~nm}$ and $30 \mathrm{~nm}$ silver NPs cause a dose-dependent decrease in viability and an increase in MCP-I and IL-8 release, which is statistically significant for the highest deposited doses. In the tri-culture model with macrophages, there is no change in pro-inflammatory cytokine release after exposure to $\mathrm{I} 5 \mathrm{~nm}$ and $30 \mathrm{~nm}$ silver NPs. The preliminary results of the present study show a dose-dependent decrease in viability and a dose-dependent increase in MCP-I and IL-8 production in the bi-culture long model without macrophages. This is in line with the results from our previous in vivo study in which exposure to silver NPs caused a dose-dependent increase in several pulmonary toxicity parameters like cell counts and pro-inflammatory cytokines in the bronchoalveolar lavage fluid (Braakhuis et al., 20I 5a). The submerged exposures, however, resulted in a dose-dependent decrease in MCP-I instead of an increase (Braakhuis et al., 20 I $5 \mathrm{~b}$ ). Similar to both the in vivo study and the submerged exposure, the observed effects after air-liquid exposure were particle size-dependent as in the bi-culture model the $15 \mathrm{~nm}$ and $30 \mathrm{~nm}$ silver NPs caused significant changes in cell viability and the release of pro-inflammatory cytokines, while $45 \mathrm{~nm}$ silver NPs did not. The previous study of Holder and Marr also showed that exposure to $50 \mathrm{~nm}, 75 \mathrm{~nm}$ and IOO $\mathrm{nm}$ silver NPs at the air-liquid interface caused no cytotoxicity and only a mild inflammatory response (Holder and Marr, 2013). Herzog et al., however, did not observe cytotoxicity after air-liquid exposure to 20 nm silver NPs (Herzog et al., 2013), although they tested higher deposited doses of silver NPs $\left(0.03\right.$ and $0.278 \mu \mathrm{g} / \mathrm{cm}^{2}$ ) compared to the present study. The difference in results between the previous and the present study might be caused by differences in the exposure system. Here, we generated silver NPs using spark generation that are later on humidified, while in the system used by Herzog et al., silver NPs are first dispersed in fluid and then nebulized into small drops resulting in deposition of a cloud of drops onto the cells (Herzog et al., 2013) that is different from the deposition by diffusion in the present study.

In conclusion, we observed a decrease in cell viability and an increase in pro-inflammatory cytokines after air-liquid exposure of a lung model without macrophages to low doses of silver NPs comparable to the results observed in vivo. Addition of macrophages resulted in a reduction of the effective dose and thereby protected the epithelial cells from the silver NPs similar to the results observed after submerged exposure. In addition, we observed a dose-dependent increase in toxicity parameters comparable to what was observed in vivo. A major advantage of air-liquid exposure is that it results in deposited doses and dose rates more similar to in vivo compared to submerged exposures in which extremely high and unrealistic exposure doses need to be used to be able to find a significant effect. As the data presented here are based on single experiments, the airliquid exposures should be repeated in the future to confirm that they better mimic the effects that occur in vivo compared to submerged exposure. 


\section{ACKNOWLEDGEMENTS}

This work was sponsored by the Quality Nano Transnational Access program of the Quality Nano Research Infrastructure. In addition, this work was supported by the project 'Integrated Risk Assessment of Nanomaterials' from the National Institute for Public Health and the Environment and by the NanoNextnl program 'Risk Analysis and Technology Assessment: Human Health Risks'. We would like to thank Christoph Schlager, Marco Mackert, Sonja Schaaf and Jolanda Vermeulen for their excellent technical assistance. 

Summarizing discussion 


\section{SUMMARIZING DISCUSSION}

Because of their small size, nanomaterials have unique physicochemical properties compared to their bulk counterparts and therefore nanomaterials are used in an increasing number of consumer products (Nanotechnologies, 20 I4). Besides beneficial properties, there are concerns that nanomaterials might introduce health risks to both workers and consumers. Inhalation is considered an important route of exposure and several studies indicate that nanomaterials can induce pulmonary toxicity after inhalation (eg. Ji et al., 2007, Pauluhn, 20II, Sung et al., 2009, Yang et al., 2008, Oberdorster et al., 2000, Warheit et al., 2007c, Warheit et al., 2007b, Pauluhn, 2009a). Given the large variety of nanomaterials and the potential hazardous properties of some nanomaterials, their risk needs to be assessed adequately.

As explained in chapter $\mathbf{1}$, the number of particles, surface area and volume of a nanomaterial may differ for the same unity of mass. Therefore, expressing the effects of nanomaterials based on the conventional mass dose will not be appropriate for risk assessment as each nanomaterial with slightly different characteristics, e.g. $20 \mathrm{~nm}$ vs. $50 \mathrm{~nm}$, spherical vs. ellipses, coated vs. non-coated, should in principle be assessed separately. By reviewing published inhalation and instillation studies in which multiple nanoparticles (NPS) differing in one physicochemical characteristic were investigated within the same in vivo study, we tried to identify trends regarding the characteristics of nanomaterials and pulmonary inflammation, as a key hazard indicator. The outcome of this review was that many physicochemical characteristics of NPs affect their lung deposition, clearance, and pulmonary response that, in combination, ultimately determine whether pulmonary inflammation will occur and to what extent. With all these characteristics affecting different stages of the events leading to pulmonary inflammation, no unifying dose metric could be identified to describe pulmonary inflammation for all nanomaterials (chapter 1). Therefore, the main goal of this thesis was to understand, assess, and implement in risk assessment the dose metric(s) that is (are) most suited for modelling the dose-effect relationship of nanomaterials for human health. Risk assessment is now mostly based on data derived from animal studies. To reduce animal testing, we also aimed to develop in vitro models that can be used to determine suitable dose metrics of nanomaterials.

\section{To achieve this goal, the following objectives were formulated:}

I. Determination of the dose metric which provides the best descriptor for doseresponse relationships of nanomaterials after inhalation exposure

2. Evaluation of in vitro models to determine suitable dose metrics of nanomaterials and their bioavailability after inhalation exposure

3. Implementation of dose metrics in the risk assessment of nanomaterials after inhalation exposure, including a testing strategy 


\section{DETERMINATION OF THE DOSE METRIC WHICH PROVIDES THE BEST DESCRIPTOR FOR DOSE-RESPONSE RELATIONSHIPS OF NANOMATERIALS AFTER INHALATION EXPOSURE}

In vivo inhalation studies were performed to determine the most suitable dose descriptor for nanomaterials after inhalation. For these studies, the relationship between external exposure and the internal dose is of importance as the dose at the site of action determines whether adverse effects occur. This internal dose should be used to determine suitable dose metrics for nanomaterials.

In chapter 2, we described the possibilities and limitations of available techniques to detect nanomaterials in biodistribution studies, i.e. to detect the internal dose. The evaluation of the presence of NPS to determine their tissue distribution is not easy. Nanoparticle presence can be identified by electron microscopy but this is a time and labour consuming technique. So, instead of determining the presence of the NPS themselves distribution studies can use elemental analysis by inductively coupled plasma mass spectrometry (ICP-MS) as indication for the tissue distribution of NPs. The use of ICP-MS in biodistribution studies provides a lot of valuable, quantitative information for the risk assessment of inorganic nanomaterials. With the classical approach of digestion and the determination of the total concentration of the element of interest, larger series of tissue samples can be analysed within a reasonable period of time, allowing for sufficient information about the distribution in various organs and body fluids of interest. New challenges and possibilities lie ahead for the application of ICP-MS in biodistribution studies, e.g. the use of single particle-ICP-MS or hyphenated techniques like field-flowfractionation (FFF)-ICP-MS. These techniques allow for a more selective determination of the presence of NPS - rather than just detection of the element - but must be further developed for routine application in this field. Additional analytical challenges involve the analysis of multi-element nanomaterials, for example silica-coated silver NPs.

In chapter 3, we presented the results of a short-term inhalation study in which the particle size was chosen as a variable to investigate its effect on the induction of pulmonary toxicity. In this study, rats were exposed via inhalation to silver NPS of I $5 \mathrm{~nm}$ and $4 \mathrm{IO} \mathrm{nm}$ to determine differences in lung deposition, tissue distribution and adverse effects. Exposure to I $5 \mathrm{~nm}$ silver NPs induced a moderate inflammatory response at 24 hours after the end of the exposure period. All the observed effects disappeared at 7 days after exposure. The most pronounced observed effect was the influx of neutrophils at 24 hours after exposure to I $5 \mathrm{~nm}$ silver NPs, which was about 175 times larger compared to the controls. In addition, we found release of pro-inflammatory cytokines, an increase in total glutathione and the presence of silver NPs inside lung cells. In contrast, the $4 \mathrm{IO} \mathrm{nm}$ particles induced none of these effects in the lung, clearly demonstrating a particle size dependent difference in the induction of pulmonary inflammation. The particle sizedependent differences can be explained by the difference in lung deposition; the smaller NPs deposit more efficiently in the alveoli compared to the larger particles (Asgharian et 
al., 2009) and by the difference in dissolution rate; the smaller particles dissolve faster compared to larger particles (Ma et al., 20I2). Using TEM, we observed substantial dissolution of the I $5 \mathrm{~nm}$ silver NPs inside lung cells. The results of this study indicate a clear size-dependent effect of silver NPS after inhalation; however, because only two different particle sizes were tested in a single dose, the data was not sufficient to derive a suitable dose metric for silver NPS.

In chapter 4, we presented a short-term inhalation study with silver NPs of four different sizes and with four different dosages. The results of this study indicated a dose-dependent increase in pulmonary toxicity parameters for all particle sizes. We could identify the surface area of silver NPs that reach the alveoli as the most suitable dose metric to describe the acute effects of silver NPs after short-term inhalation. The study highlights the importance to measure the internal dose; when using the alveolar dose instead of the external concentration in air, the particle surface area gives almost completely overlapping dose-response curves for the different particle sizes, while this is not the case when the external concentration in air is used. The dose metric can be used to predict the effects of similar, uncoated silver NPs of other sizes that not have been tested. Regarding long-term inhalation or chronic effects, the dose descriptor might be different since the kinetics of the silver NPS and dissolution behaviour will play a role in the distribution of silver to other organs or tissues of the body where they might induce toxicity that is not directly correlated to the internal alveolar dose. For NPs that differ from the silver NPs tested in this study, for example other chemical composition, shape or coating, the most suitable dose descriptor can be different because of differences in kinetics and mechanisms that might induce toxicity.

In conclusion, for describing the dose-response relationship of silver NPs after inhalation exposure, the surface area of the silver NPs that reach the alveoli provides a suitable dose metric. To measure the internal dose, that is needed to estimate the amount of particles that reach the alveoli, different ICP-MS techniques can be used.

\section{EVALUATION OF IN VITRO MODELS TO DETERMINE SUITABLE DOSE METRICS OF NANOMATERIALS AND THEIR BIOAVAILABILITY AFTER INHALATION EXPOSURE}

Although animal studies provide unique information on the distribution and effects of nanomaterials, the use of animals is ethically debatable and should be reduced. In addition, given the large variety in nanomaterials, it is impossible and economically not feasible to test all of them using in vivo studies. Therefore, many in vitro models have been developed to test the distribution and effects of nanomaterials.

In chapter 5, we evaluated the progress and future of lung in vitro models to study the translocation of nanomaterials. Many in vitro models have been developed to test the 
translocation of NPs because there are concerns that NPS would unintentionally become systemically available in the human body across biological barriers. In vitro models of varying complexity are available, ranging from single cell type monolayer to multi cell (3D) models. Only a few studies are available that allow comparison of the in vitro translocation to in vivo data. At the current state-of-the-art the in vitro models do not yet allow prediction of absolute transfer rates but they do support the definition of relative transfer rates and can thus help to reduce animal testing by setting priorities for subsequent in vivo testing. Simple in vitro models consisting of a single cell type have the advantage that they can be used on a large scale, while more complex models have the advantage that they are physiologically more similar to the human situation. Although suitable in vitro models still have to be validated for studying translocation in risk assessment, the current strategy to improve the models to more closely mimic the human situation by using co-cultures of different cell types and microfluidics approaches, are deemed essential.

In chapter 6, we evaluated the use of insert membranes differing in pore size and membrane material to study the in vitro translocation of fluorescent polystyrene NPS with different surface charges. The translocation of polystyrene NPs differs between the different insert membranes with the larger pore sizes showing a higher translocation compared to the smaller pore sizes and the polycarbonate inserts showing a higher translocation compared to the polyester inserts. Regarding the surface charge of the polystyrene NPs, the positively charged polystyrene NPs showed the highest translocation and cytotoxicity in the lung model at lower concentrations compared to the neutral and negatively charged polystyrene NPS. Fluorescent NPs have the advantage of being easily detectable at low costs. However, for many of the tested nanoparticle concentrations, the translocation was below the detection limit of the fluorescence signal even when testing insert membranes without cells. For studying translocation in vitro, the ability of NPs to cross the insert membranes should be checked before starting experiments. In addition, more sensitive detection techniques like fluorescent correlation spectroscopy, ICP-MS or radiolabeling should be used to allow comparison of in vitro translocation to in vivo data. Once a suitable in vitro model and detection method is available to test the translocation of NPS, the results can be used to identify relative transfer rates of NPS with different physicochemical characteristics and thus help priority setting for nanoparticle testing.

In chapter 7 , we showed that when using a simple monolayer of lung epithelial cells, the most suitable dose metric to describe the effect of silver NPs is their particle surface area, similar to the dose metric found in vivo. Although the dose metric of silver NPs is the same between the monolayer of lung epithelial cells in vitro and the in vivo study, the exposure concentrations in vitro are a factor of 6 to 6000 times higher. Increasing the complexity of the in vitro model by adding alveolar macrophages and culture the model on Transwell ${ }^{\circ}$ inserts results in less correlation with in vivo data. A possible explanation for this remarkable result is that in vivo there are normally a fixed number of macrophages 
in the lungs and additional macrophages are recruited upon an exposure. The fixed number of macrophages is very low, about one macrophage per alveoli (Gordon and Read, 2002, Kopf et al., 2015). When inhaled NPs deposit in the alveoli, they interact with the epithelial cells that are triggered to produce pro-inflammatory cytokines to recruit macrophages (Stone et al., 2007). There is thus most likely an exposure to NPs of the epithelial cells before macrophages are recruited, in vivo. The alveolar macrophages reduce the effective dose of the NPs that reach the epithelial cells by phagocytosis of the NPs, which results in a lower acute effect. Therefore, for studying the acute pulmonary toxicity of NPs, using a monolayer of epithelial cells better predicts the in vivo outcome compared to a co-culture model with macrophages. However, for long-term effects the macrophages are of importance because NPS can dissolve over time in lysosomes leading to the leakage of ions which can damage the lungs. In addition, after long-term exposure to NPS, the clearance capacity of alveolar macrophages can be overwhelmed resulting in lung particle overload (Pauluhn, 20 I I, Morrow, I992), which may result in sustained inflammation, fibrosis or even the induction of lung tumours (ILSI, 2000). To study the pulmonary effects under overload conditions in vitro, macrophages are an essential element in the model.

In chapter 8, we presented the results of a preliminary study exposing an in vitro lung model (with and without the addition of macrophages) to silver NPs at the air-liquid interface to determine whether exposure doses and effects at the air-liquid interface correlate better to the in vivo situation compared to submerged exposure. In the lung model without macrophages, exposure to silver NPS at the air-liquid interface caused a dose-dependent decrease in cell viability and a dose-dependent increase in MCP-I and IL-8 similar to in vivo, while we observed a dose-dependent decrease of MCP-I release after submerged exposure. Similar to the submerged exposures, addition of macrophages resulted in a reduction of the effective dose and a protection of the epithelial cells. The doses at which significant changes in pulmonary toxicity parameters were observed after air-liquid exposure $\left(0.0025-0.02 \mu \mathrm{g} / \mathrm{cm}^{2}\right)$ were similar to the in vivo deposited doses (0.000 I - $0.005 \mu \mathrm{g} / \mathrm{cm}^{2}$ ) while under submerged conditions extremely high doses were needed to observe significant effects $\left(3-30 \mu \mathrm{g} / \mathrm{cm}^{2}\right)$. The data from the air-liquid exposures result from single experiments and should be repeated in the future to confirm that air-liquid exposure better mimics the effect-doses in vivo compared to submerged exposure.

In conclusion, many different in vitro lung models are available that still have to be validated for studying translocation of NPs. Most in vitro models consist of cells cultured on Transwell ${ }^{\circ}$ insert membranes. The pore size and the membrane material of these inserts influence the translocation rate of NPs. Since the in vivo translocation of NPS is low $(<0.5 \%)$, sensitive detection techniques are needed to allow comparison of in vitro translocation to in vivo data. For studying the effects of NPs after inhalation, a simple monolayer of lung epithelial cells might be suitable as using this monolayer the same dose metric - particle surface area of silver NPs - could be found compared to in vivo. A 
disadvantage of this submerged model is that high doses are needed to find a significant effect. Using air-liquid exposure models, the effect-doses are more similar to in vivo.

\section{IMPLEMENTATION OF DOSE METRICS IN THE RISK ASSESSMENT OF NANOMATERIALS AFTER INHALATION EXPOSURE}

In this thesis, we identified the particle surface area that reaches the alveoli as a suitable dose metric to describe the acute effects of uncoated silver NPs after inhalation both in vivo and in a monolayer of lung epithelial cells in vitro. Particle surface area of silver NPS that reach the alveoli is a suitable dose descriptor for the pulmonary toxicity of silver NPs because I) The alveoli are the site of the observed toxicity, 2) Silver NPs of different sizes have a different deposition pattern in the lungs, including the alveoli (Asgharian et al., 2009), 3) The size of silver NPs influences cellular responses, including uptake (Zhang et al., 20I4); smaller NPs enter cells more easily than larger ones (Liu et al., 20 Io), 4) Silver NPs can release silver ions from their surface (Ma et al., 20 I 2, Leo et al., 20I3, Stebounova et al., 20 I I b, Kent and Vikesland, 20 I 2, Zook et al., 20 I I), possibly causing the observed effects, as has been reported previously (Pratsinis et al., 2013, Beer et al., 20I2, Wang et al., 20I4b, Lubick, 2008, Park et al., 20I0), and 5) Silver NPs might cause their effects by the direct contact of their surface with the epithelial cells.

The dose descriptor was identified by first modelling the amount of particles that reach the effect site, which are the alveoli in the case of inhalation exposure. Subsequently, the effects were related to different dose metrics of the alveolar dose. It is important to distinguish particle distribution, kinetics and toxicity as some particle characteristics may have a major impact on distribution and kinetic processes, whereas other particle characteristics are important for inducing an effect. As a consequence, when the dose metric is investigated in view of external dose instead of the dose at the effect site, different findings may occur, based on the interaction between kinetics and toxicity.

The identified dose metric can be used in the risk assessment of silver NPs to predict the acute effects of uncoated silver NPs of different sizes that have not been tested yet. For example the release of MCP-I in the lung fluid was increased by $20 \%$ after exposure to $\mathrm{I} .7 \times 10^{9} \mathrm{~nm}^{2}$ silver NPs per $\mathrm{cm}^{2}$ of alveoli, irrespective of the particle size, while the doses are different based on mass (0.000053 $\mu \mathrm{g} / \mathrm{cm}^{2}$ for the $\mathrm{I} 8 \mathrm{~nm}$ and $0.00010 \mu \mathrm{g} / \mathrm{cm}^{2}$ for the $34 \mathrm{~nm}$ NPS). For $74 \mathrm{~nm}$ silver NPs that not have been tested in our in vivo study, the release of MCP-I will probably also increase by $20 \%$ after exposure to $\mathrm{I} .7 \times 10^{9} \mathrm{~nm}^{2}$ silver NPs per $\mathrm{cm}^{2}$ of alveoli, while the mass dose will be different $\left(0.00022 \mu \mathrm{g} / \mathrm{cm}^{2}\right)$. For the risk assessment of NPS that differ in other characteristics like chemical composition, coating or surface charge, the dose metric should be determined - in studies that test NPS that vary in a single characteristic - to be able to predict the effects of other NPs that not have been tested. 


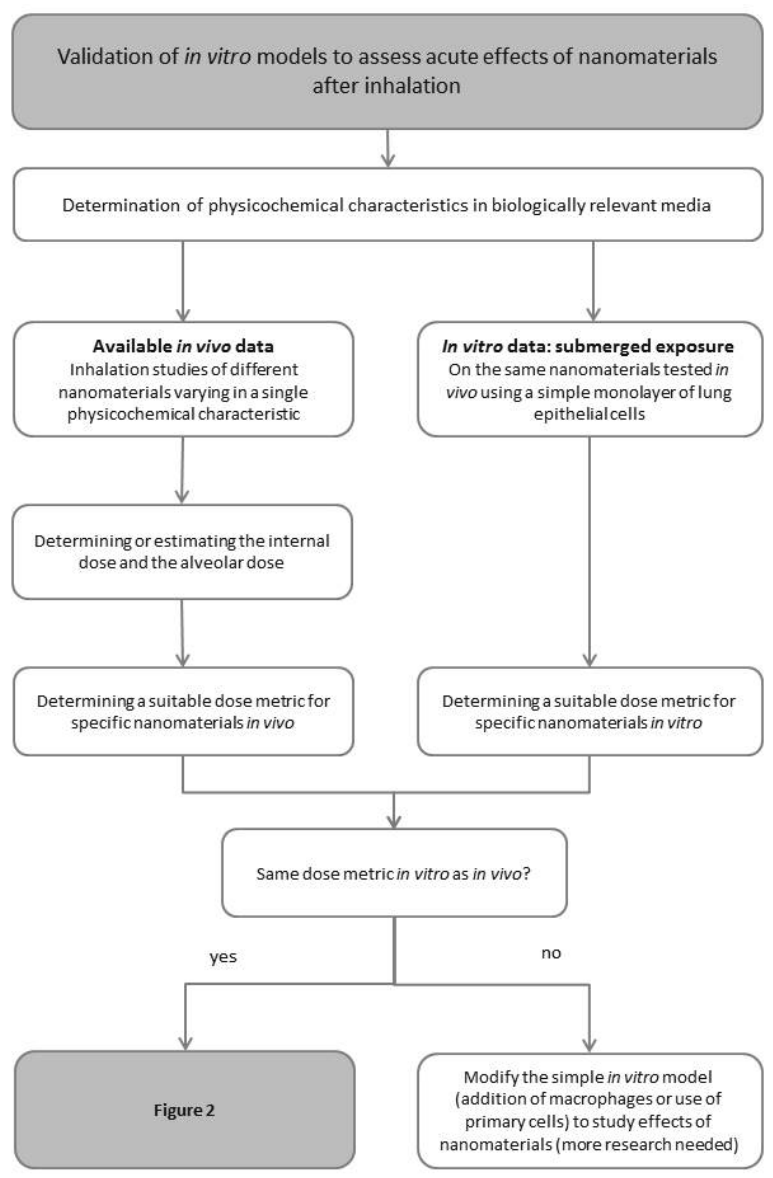

Figure 1. Validation of in vitro models to assess the acute effects of nanomaterials after inhalation.

\section{TESTING STRATEGY TO ASSESS THE ACUTE EFFECTS OF NANOMATERIALS AFTER INHALATION}

For the risk assessment of nanomaterials, information is needed on both the hazard of nanomaterials and the exposure to nanomaterials. Hazard identification can be accomplished using in vivo and in vitro studies as has been shown in the results from this thesis. The use of animals is ethically debatable and - giving the large number of nanomaterials - not feasible. Therefore, the use of in vitro methods should be implemented in the risk assessment of nanomaterials. Here, we present a testing strategy to identify the hazard of nanomaterials after inhalation in which in vitro methods and dose metrics play a central role (figure I and 2). This testing strategy is designed to identify the acute 
effects of nanomaterials that have not been tested (sufficiently) previously and newly developed ones, without the use of animal studies. For nanomaterials that have been tested in animal studies, the available data can be used to derive effect doses that can be compared to the exposure in humans to perform risk assessment for these nanomaterials. However, for new or not sufficiently tested nanomaterials, animal studies should be avoided and the following testing strategy can be used to assess the acute effects of nanomaterials after inhalation.

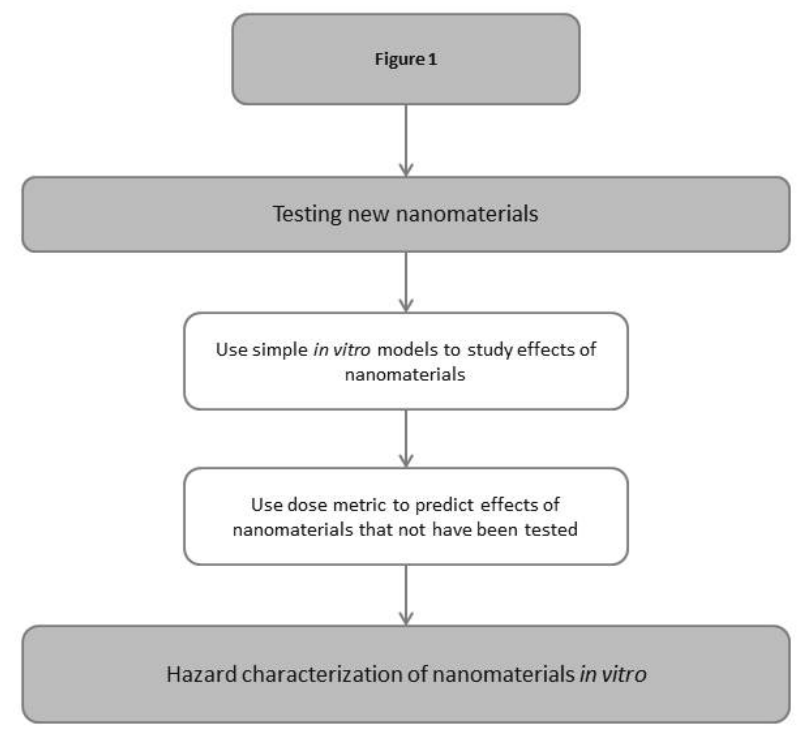

Figure 2. Testing strategy to assess the acute effects of nanomaterials after inhalation.

The first important step is to characterize the nanomaterials in biologically relevant media (e.g. agglomerate size, shape, surface reactivity) (Walser and Studer, 20 I 5, Braakhuis et al., 20I 4b). Macromolecules in biological fluids can change nanomaterial characteristics and thereby influence cellular uptake and effects. The nanomaterial characteristics at the site of exposure should be linked to the observed effects in order to identify the toxicitydetermining characteristics. Since there are no validated in vitro models available for the hazard assessment of nanomaterials, the second step in our testing strategy is to validate an in vitro model using in vivo data (figure I). We propose that a simple monolayer of lung epithelial cells can be used as we showed that using this model we could identify the same dose metric as in vivo for the acute effects of uncoated silver NPs (chapter 7).

For the validation, available in vivo data should be collected in which nanomaterials that differ in a single physicochemical characteristic have been tested in the same experimental setup. If possible, the internal dose and the alveolar dose should be determined or modelled to correlate the dose at the effect site to the observed effects. The data can be used to identify a suitable dose metric for these nanomaterials in vivo. By testing the 
same nanomaterials in the simple lung model as have been tested in available in vivo studies, the in vitro dose metric can be determined. If the dose metric is the same between in vivo and in vitro, the simple monolayer proves to be a suitable model to use in this testing strategy for deriving an appropriate dose metric. If there are differences between the in vivo and in vitro dose metric, more complex submerged in vitro models should be used as for those particular nanomaterials the monolayer of lung epithelial cells is not a suitable model. Possibilities are to use primary lung cells that might be more sensitive to detect acute toxicity induced by nanomaterials. Other possibilities are to include endothelial cells, macrophages and/or dendritic cells (Braakhuis et al., 20 I 5 c) or to use microfluidic systems instead of conventional static systems. Microfluidic systems use a continuous medium flow that enables a continuous monitoring of adverse effects and maintains a stable nanoparticle suspension during exposure resulting in physiologicallyrelevant measurement conditions (Mahto et al., 2015). For most nanomaterials, we expect that the acute effects will be related to lung epithelial damage and therefore we expect that the simple monolayer of lung epithelial cells under submerged conditions will prove to be a suitable model. However, for some nanomaterials that do not induce acute effects, and for long-term and repeated exposures, the monolayer of epithelial cells will probably not be a suitable model as the effects will probably not be related to acute epithelial damage, and kinetics and secondary effects play a more important role. For these conditions, more research is needed to find suitable in vitro models. If the epithelial monolayer proves to be a suitable in vitro model to study the acute effects of nanomaterials after inhalation, new chemically similar nanomaterials with variations in the same physicochemical property can then be tested in vitro only (figure 2). In this thesis, this strategy has been successful applied to silver NPs of different sizes. If the strategy turns out to be equally successful for nanomaterials of different chemical compositions and physicochemical properties, appropriate dose metrics can be identified using in vitro studies only (figure 2). By identifying the suitable dose metric, the in vitro data can be used to predict the effects of nanomaterials that not have been tested while avoiding animal studies. The information can be used for the hazard characterization of nanomaterials.

As we showed in chapter 8, the delivered dose used in in vitro studies under submerged conditions is magnitudes larger compared to the effect doses in vivo and after air-liquid exposure. Ideally, we would test all nanomaterials in an air-liquid exposure system to determine effect doses that are more realistic to the in vivo situation. When validated air-liquid models would be available, these models could be used to identify realistic effect-doses of nanomaterials after inhalation exposure that can directly be used in the risk assessment of nanomaterials. However, before air-liquid models can be used, they should first be optimized and validated by testing the same nanomaterials in an air-liquid exposure system as have been tested in vivo and comparing the results. In addition, there are not many air-liquid exposure systems available and the number of exposure modules is limited, making air-liquid exposure more labour-intensive and time consuming compared to submerged exposure in for example 96-wells plates. 
It is important to note that the dose metric not only depends on the characteristics of the NPs but also on the experimental design and measured endpoints. Therefore, we recommend that these variables are also taken into account. When comparing the dose metrics between different studies, the same endpoint should be compared. In addition, when different dose metrics of the same nanomaterial are identified in different experimental setups, an experimental design that mimics inhalation more closely such as in vivo nose-only exposure or air-liquid exposure is leading compared to intratracheal instillation or submerged exposure.

\section{FUTURE PERSPECTIVES}

Besides identification of a suitable dose metric, data from in vivo and in vitro studies on nanomaterials that vary in a single characteristic could in future be used to develop QSARS to predict the effects of new nanomaterials. For this purpose, a large amount of data is required, which can be combined into a data set to carry out multivariate analysis to determine potential combinations of characteristics that are important in affecting pulmonary inflammation. Information on the toxicity-determining characteristics of nanomaterials could be used to help the development of new nanomaterials that are safe-by-design.

The strategy presented above can be used for the risk assessment of acute effects of nanomaterials after acute/short-term exposure because the included in vitro methods use a single acute exposure that varies between 4 and 24 hours. However, there are concerns that a life-long exposure to low concentrations of nanomaterials might cause health effects that might show up after years of exposure. Most toxicity assays (both in vivo and in vitro) focus on the acute exposure and effects because of money and time constraints. As discussed above in the testing strategy, the acute effects of nanomaterials are probably (related to) epithelial damage; a simple monolayer of epithelial cells might be sufficient to determine the dose metric of several different nanomaterials or to group many nanomaterials according to their hazard in vitro. Probably, the effects of nanomaterials after repeated or long-term inhalation exposures, are different from those observed after acute exposure because clearance mechanisms and secondary effects play a more important role. Therefore, a simple monolayer of epithelial cells will probably not be sufficient to determine the effects of nanomaterials after repeated or long-term exposure. In addition, in vitro assays usually are based on immortalized cell lines that are not suitable to use for repeated or long-term exposures as they keep on replicating. A solution might be the use of primary cells that can be cultured for a longer time period. Primary cells have a more differentiated phenotype compared to cell lines, however, their isolation is experimentally challenging, they differ from batch to batch, and are difficult to maintain in a well-differentiated phenotype. For testing repeated exposures, microfluidic systems are currently being developed that are able to test the effects of nanomaterials under physiologically relevant conditions using a 
continuous medium flow. The continuous flow delivers nutrients and removes waste and allows a homogenous exposure to nanomaterials that alters their uptake compared to conventional static exposure (Mahto et al., 2015). For the lungs, an air-liquid exposure system on a chip has been developed that uses mechanical strain to mimic the motions during breathing that enhances the uptake of nanomaterials (Huh et al., 2010). These microfluidic devices in combination with primary cells are promising to test the effects repeated and long-term exposure to nanomaterials.

In conclusion, given the increasing number of nanomaterials, risk assessment of nanomaterials after inhalation exposure is of importance. It is not feasible to test each nanomaterial with slightly different characteristics to identify their hazard. Here, we identified the particle surface area that reaches the alveoli as a suitable dose descriptor to describe the acute effects of silver NPs both after in vivo inhalation and in lung epithelial cells in vitro. For other nanomaterials that differ in a single characteristic, a suitable dose metric can be identified in a similar way, eventually using the lung epithelial cells to reduce animal testing. Information on the dose metrics can be used to predict the effects of nanomaterials that have not been tested. Finally, the information can be used to categorize nanomaterials according to their hazard which can be compared to the exposure to nanomaterials to assess the acute risks. For repeated or long-term exposure of nanomaterials, physiologically relevant in vitro systems like microfluidics should be further optimized. 




\section{Valorisation paragraph Nederlandse samenvatting References Dankwoord List of publications Curriculum vitae}





\section{Valorisation paragraph}

This thesis focuses on the effects of nanomaterials after inhalation. In this paragraph, the value of this thesis for the society and economy is discussed.

In the society, there are concerns that nanotechnology poses risks to human and environmental health. For many people nanotechnology and related terms are difficult to understand as nanomaterials and nanotechnology cannot be seen by the naked eye. In the meantime, nanomaterials are used in a growing number of consumer products because of their unique physicochemical properties that are related to their small size. These unique properties can be beneficial - for example nanomaterials are used to develop efficient solar cells, dirt-repellent textiles, anti-cancer drugs and fast computers - or they might introduce health risks. There is need for adequate and efficient risk assessment of nanomaterials to keep up with the growing number of products containing nanomaterials. Currently, there are 292 personal care products on the market that claim to contain nanomaterials (Nanotechnologies, 20 I4). People can be exposed to these products daily, while the safety of these nanomaterial containing products is not tested. There are many concerns in the society that a long-term exposure to nanomaterials will induce adverse effects in the future; we just do not know this yet. Especially inhalation of nanomaterials is of concern given the vulnerability of the lungs and the experiences in the past with asbestos. The challenge is to assure the safety of nanomaterials and nanotechnology without hampering the development of new products and services of which the society can benefit.

At the moment, each nanomaterial with slightly different characteristics, e.g. $20 \mathrm{~nm}$ vs. $50 \mathrm{~nm}$, spherical vs ellipses, coated vs. non-coated, should in principle be assessed separately. Given the large number and variety of nanomaterials, it is not feasible to test each nanomaterial with slightly different characteristics to identify their hazard. In this thesis, we identified the particle surface area that reaches the alveoli as a suitable dose descriptor to describe the acute effects of silver nanoparticles both after in vivo inhalation and in lung epithelial cells in vitro. The identified dose metric can be used in the risk assessment of silver nanoparticles to predict the acute effects of uncoated silver nanoparticles of different sizes that have not been tested yet, thereby reducing the number of studies needed to assess the risks of nanomaterials. For example the release of a pro-inflammatory cytokine in the lung fluid was increased by $20 \%$ after exposure to $1.7 \times 10^{9} \mathrm{~nm}^{2}$ silver nanoparticles per $\mathrm{cm}^{2}$ of alveoli, irrespective of the particle size. For $74 \mathrm{~nm}$ silver nanoparticles that not have been tested in our in vivo study, the release of this marker will probably also increase by $20 \%$ after exposure to $1.7 \times 10^{9} \mathrm{~nm}^{2}$ silver nanoparticles per $\mathrm{cm}^{2}$ of alveoli. For the risk assessment of nanomaterials that differ in other characteristics like chemical composition, coating or surface charge, the 
dose metric can be identified - in studies that test nanomaterials that vary in a single characteristic - to be able to predict the effects of other nanomaterials that not have been tested. In this way, information on the hazard of nanomaterials can be efficiently gathered without testing all nanomaterials separately.

To perform risk assessment of nanomaterials without stopping innovation in the field of nanotechnology, there is need for new strategies that efficiently gather hazard information on nanomaterials. Next to this, a clear communication between industry, safety assessors and legislators is needed. Risk assessment of nanomaterials should not be based on a checklist of experiments that are required for other chemicals. Efforts should be made to optimize experiments for nanomaterials by focusing on nanomaterial characterization, dosimetry and the development of alternative methods. In order to make this work, industries should be transparent about the use of nanomaterials in products and possible exposure to both workers and consumers. In addition, safety assessors should be trained on nanomaterial characteristics and behaviour to be able to use this knowledge in the risk assessment of nanomaterials. Finally, legislators should be more flexible in accepting risk assessment information and strategies that are not based on checklists of (animal) experiments that are required for conventional chemicals. In this thesis, we present a new testing strategy to assess the acute effects of nanomaterials after inhalation without using animals. This testing strategy can help to speed up the risk assessment process and thereby the development of new nanomaterials and nanotechnology-enabled products.

To inform people about the results of this thesis, many chapters are also presented in peer reviewed journals, at conferences and in a short movie. Peer reviewed journals are mostly read by colleagues within the nanotoxicology research field. At conferences, oral presentations and poster presentations reach a broader audience within the nanomaterial and toxicology field. Especially within NanoNextNL - which is a consortium of more than one hundred companies, universities, knowledge institutes and university medical centres, aimed at research into micro and nanotechnology - there was great interest in risk assessment of nanomaterials. NanoNextNL funds many PhD projects that all are required to include risk assessment in their thesis. During a risk assessment and technology assessment (RATA) course organized by NanoNextNL, the research present in this thesis was used to explain the importance of risk assessment of nanomaterials to other phD candidates that are involved in developing new nanomaterials and technologies. By including risk assessment in the development of nanomaterials and nanotechnology, products can be developed that are safe by design. This means that information on toxicity-determining characteristics of nanomaterials can be included in the development of new products. For example, the use of fibre shaped nanomaterials in sprays will be avoided as they are more hazardous compared to spherical particles. In addition, new nanomaterials can be tested in toxicity assays and subsequently modified until they induce minimal or no toxicity. NanoNextNL also encouraged collaboration between universities, institutes and industry resulting in sharing of knowledge between experts in the field of toxicology, risk assessment, physics, and chemistry. These collaborations will help future projects on 
nanomaterials to include the expertise form different fields and will aid the development of nanomaterials that are safe by design. Next to this, the results of this thesis will be used as a case study in future workshops on dosimetry to inform other researchers about the importance to use the appropriate dose metric to describe the effects of nanomaterials.

This thesis also resulted in a close collaboration with the Karlsruhe Institute of Technology (кIT). The QualityNano (QNano) Transnational Access program of the QNano Research Infrastructure sponsored visits to KIT. This gave us the opportunity to work with a newly developed exposure system that is able to expose cells in vitro to nanoparticles at the airliquid interface under temperature and humidity controlled circumstances. For studying the effects of nanomaterials after inhalation, such innovative systems are essential as they can mimic the in vivo inhalation more closely compared to conventional submerged exposures, especially regarding the deposited doses and dose rates.

To inform the general public about research that is performed on the toxicity of nanomaterials after inhalation, a short movie was released via the website of the National institute for Public Health and the Environment (RIVM):

http://www.rivm.nl/Documenten_en_publicaties/Algemeen_Actueel/Multimedia/ Infectieziekten/Wetenschapscommunicatie/Inademing_van_nanomaterialen/ Download/Inademing_van_nanomaterialen

This movie was specially made for the lay public as part of the project 'Jonge onderzoekers in beeld'. The purpose of this project was to provide opportunity for phD students to present their research professionally and clearly to the general public, and to provide information to the public about the relevance to society of research conducted at the RIVM.

There are no specific regulatory frameworks or guidance documents for the risk assessment of nanomaterials (yet). In this thesis, a novel testing strategy is presented to assess the acute effects of nanomaterials after inhalation without using animals. If this testing strategy proves to be valid, a large number of nanomaterials can be tested. The testing strategy presented in this thesis focuses on the acute effects of nanomaterials after inhalation. A similar strategy can be designed for other exposure routes and effects, for example after oral exposure. By first determining the appropriate dose metric both in vivo and in vitro, preferably using available in vivo data, in vitro models can be validated. Next, these in vitro models can be used to determine the hazard of new nanomaterials after for example oral exposure. Information on the hazard of nanomaterials is essential for their risk assessment. In addition, the information can also be used to group nanomaterials by their hazard in order to find characteristics that are linked to hazard. This will help to develop and produce nanomaterials that are safe by design. This is beneficial to the economy as the development of nanomaterials and nanotechnology enabled products is a growing market. The society also benefits as safe nanomaterials can be used to develop beneficial products like sunscreens, medicines and computer technology while exposure to potentially harmful nanomaterials is minimized. 



\section{Nederlandse samenvatting}

\section{INLEIDING}

Nanotechnologie is het gebruik van zeer kleine stoffen of structuren waarmee nieuwe producten en toepassingen gecreëerd worden. De term 'nano' komt van het Griekse woord vâvos, dat 'dwerg' betekent. 'Nano' wordt gebruikt om de grootte van kleine structuren aan te geven. Volgens de definitie van de Europese Commissie spreken we van een nanomateriaal als het deeltje in één of meer dimensies kleiner is dan $100 \mathrm{~nm}$ (Commission, 20II). Één nanometer is een miljardste meter: I $\mathrm{nm}$ is 80.000 keer kleiner dan de diameter van een menselijke haar en een vingernagel groeit ongeveer I $\mathrm{nm}$ per seconde. De term nanomaterialen is een container begrip. Het enige wat nanomaterialen gemeenschappelijk hebben is dat ze allemaal klein zijn. Daarnaast kunnen nanomaterialen verschillen in allerlei eigenschappen. Ze kunnen verschillen in grootte, vorm, kleur, coating en chemische samenstelling. Doordat nanomaterialen zeer klein zijn, hebben ze een relatief groot oppervlak, waardoor ze bijzondere eigenschappen hebben. Ze worden bijvoorbeeld gebruikt om efficiënte zonnecellen, vuilafstotend textiel, medicijnen tegen kanker en snelle computers te maken.

Er zijn nu ruim I 500 consumenten producten waarvan de makers beweren dat deze nanomaterialen bevatten (Nanotechnologies, 20 I4). Hiervan zijn ongeveer 300 producten voor de persoonlijke verzorging. De verwachting is dat dit aantal snel zal stijgen, nu er wereldwijd veel onderzoek wordt gedaan naar nieuwe toepassingen van nanomaterialen. De stijging in het aantal producten dat nanomaterialen bevat roept de vraag op of deze materialen gevaarlijk kunnen zijn voor werknemers, consumenten en/of het milieu.

\section{RISICOBEOORDELING VAN NANOMATERIALEN}

Om de mogelijke risico's van nanomaterialen voor de gezondheid te onderzoeken, is er informatie nodig over zowel de blootstelling aan als mogelijke nadelige effecten van nanomaterialen. In deze thesis ligt de focus op mogelijke risico's van nanomaterialen na inademing. Nanomaterialen kunnen namelijk ingeademd worden tijdens productie of bij gebruik ervan in sprays (Oomen et al., 20I I, Wijnhoven et al., 20I0). Bovendien zijn de longen gevoeliger dan bijvoorbeeld de huid. Een aantal onderzoeken naar de effecten van nanomaterialen na inademing laat zien dat sommige nanomaterialen ontstekingen in de longen kunnen veroorzaken (eg. Ji et al., 2007, Pauluhn, 20 I I, Sung et al., 2009, Yang et al., 2008, Oberdorster et al., 2000, Warheit et al., 2007c, Warheit et al., 2007b, Pauluhn, 2009a). 
Binnen de risicobeoordeling van stoffen en nanomaterialen is dosimetrie belangrijk. Dosimetrie is de bepaling van de dosering. Meestal wordt de dosering uitgedrukt in massa (gram) per eenheid lichaamsgewicht $(\mathrm{kg})$ of massa per volume in lucht of water ( $\mathrm{L}$ of $\left.\mathrm{m}^{3}\right)$. Voor oplosbare stoffen bestaat er een vast getal voor het aantal opgeloste moleculen/ atomen per massa eenheid: de constante van Avogadro (6.02214179 $\left.\times \mathrm{IO}^{23} \mathrm{~mol}^{-1}\right)$. Voor nanomaterialen bestaat een dergelijk vast nummer niet, omdat nanomaterialen kunnen verschillen in grootte, vorm, lading en coating. Het aantal deeltjes, de totale oppervlakte en het volume van een nanomateriaal kan verschillen ook al is de massa gelijk. Bij een gelijke massa gedragen nanomaterialen zich anders dan hetzelfde materiaal in de 'bulk' vorm. Verschillende studies hebben aangetoond dat massa inderdaad niet geschikt is als dosismaat om het effect van nanomaterialen te beschrijven (Oberdorster et al., 2000, Stoeger et al., 2006, Horie et al., 2012, Duffin et al., 2007, Kobayashi et al., 2009, Oberdorster et al., 2005a). Op basis van een dosis uitgedrukt in massa is het risico van nanomaterialen anders dan van niet-nanomaterialen.

Als massa geen geschikte dosismaat is om de blootstelling aan nanomaterialen te beschrijven, wat is dan een geschikte dosismaat? En van welke deeltjeskarakteristieken is de dosismaat afhankelijk?

In hoofdstuk I beschrijven we de invloed van verschillende deeltjes-karakteristieken op het ontstaan van ontstekingen in de longen door gebruik te maken van gepubliceerde studies. Verschillende karakteristieken bepalen of nanomaterialen diep in de longen terecht komen, hoe lang het duurt om de deeltjes op te ruimen en of er een reactie optreedt in de longen. Het was niet mogelijk om één eigenschap of dosismaat te vinden die het ontstaan van effecten in de longen kan verklaren en beschrijven voor alle nanomaterialen. Daarom is het doel van deze thesis om voor de risicobeoordeling van nanomaterialen een geschikte dosismaat te vinden die de dosis-effect relatie tussen nanomaterialen en de gezondheid kan beschrijven. Risicobeoordeling is nu vooral gebaseerd op het gebruik van data uit dierexperimenten. Om het aantal dierstudies verder terug te dringen heeft deze thesis ook als doel om alternatieve in vitro modellen te ontwikkelen die gebruikt kunnen worden om een geschikte dosismaat te vinden.

Om bovenstaand doel te bereiken zijn de volgende doelstellingen geformuleerd:

I. Het bepalen van de meest geschikte dosismaat om de dosis-response relatie tussen nanomaterialen en gezondheid te beschrijven na inademing

2. Evaluatie van het gebruik van in vitro modellen om een geschikte dosismaat voor nanomaterialen te vinden en om de bio-beschikbaarheid te bepalen na inademing

3. Implementatie van de geschikte dosismaat in de risicobeoordeling van nanomaterialen na inademing, inclusief een teststrategie 


\section{HET BEPALEN VAN EEN GESCHIKTE DOSISMAAT VOOR NANOMATERIALEN}

In zowel dierstudies als in vitro studies is de relatie tussen de dosis die toegediend wordt en de dosis die daadwerkelijk op de plaats van een effect komt, de interne dosis, belangrijk. Deze interne dosis bepaalt of er effecten optreden of niet.

In hoofdstuk 2 worden de mogelijkheden en beperkingen van huidige technieken besproken die gebruikt worden om nanomaterialen te detecteren in weefsels, dus om de interne dosis te bepalen. De evaluatie van de aanwezigheid van nanomaterialen in weefsels is niet gemakkelijk. Nanodeeltjes kunnen geïdentificeerd worden met behulp van elektronen microscopie, maar dit is een tijdrovende techniek. Daarom wordt vaak inductively coupled plasma mass spectrometry (ICP-MS) gebruikt om de aanwezigheid van het element te detecteren in plaats van het deeltje zelf. In meerdere monsters kan tegelijk worden gemeten hoeveel er van een bepaald element aanwezig is. Op deze manier kan de verdeling van nanomaterialen over verschillende organen in kaart worden gebracht. De ICP-Ms techniek wordt steeds verder geoptimaliseerd om ook deeltjes te kunnen detecteren in plaats van alleen het element. Andere uitdagingen zijn de analyse van nanomaterialen die uit meerdere elementen bestaan, zoals zilver nanodeeltjes met een silica coating.

In hoofdstuk 3 worden de resultaten van een studie gepresenteerd waarin we de invloed van deeltjesgrootte op de effecten na inademing hebben onderzocht. In deze studie werden ratten via inademing blootgesteld aan dezelfde massa zilver nanodeeltjes van is $\mathrm{nm}$ en 4IO $\mathrm{nm}$. Na blootstelling aan de kleine I $5 \mathrm{~nm}$ zilver nanodeeltjes zagen we een milde ontstekingsreactie in de longen van de ratten. $\mathrm{Na}$ een week was er geen reactie meer te zien. De blootstelling aan 4IO nm zilver deeltjes veroorzaakte geen effecten in de ratten. De grootte van de deeltjes had dus invloed op de gevonden effecten. Dit kan verklaard worden uit het feit dat kleine nanodeeltjes dieper in de longen terecht komen in vergelijking met grotere deeltjes. Daarnaast lossen de kleine nanodeeltjes sneller op dan de grotere deeltjes. Met behulp van transmissie elektronen microscopie zagen we in longcellen dat de I $5 \mathrm{~nm}$ deeltjes voor een groot deel waren opgelost tot een grootte $<5 \mathrm{~nm}$. Deze studie laat zien dat er een duidelijk verband is tussen deeltjesgrootte en de effecten na inademing, maar de resultaten zijn nog niet voldoende om een geschikte dosismaat voor zilver nanodeeltjes te bepalen.

In hoofdstuk 4 worden de resultaten van een studie beschreven waarin ratten werden blootgesteld aan zilver nanodeeltjes van vier verschillende groottes en in verschillende doseringen. Voor alle deeltjesgroottes zagen we een dosis-afhankelijke toename in longtoxiciteit parameters. Door de data op verschillende manieren te analyseren konden we een geschikte dosismaat voor zilver nanodeeltjes identificeren waarbij de verschillende dosis-response curves van de verschillende deeltjes elkaar overlappen. De totale oppervlakte van de zilver nanodeeltjes die de kleinste longblaasjes (alveoli) bereikten bleek een geschikte dosismaat voor de dosis-response relatie van zilver 
nanodeeltjes na inademing. Deze studie laat zien dat het belangrijk is om de interne dosis te meten; wanneer de dosis in de longblaasjes (alveolaire dosis) wordt gebruikt in plaats van de externe dosis vallen de dosis-response curves van de verschillende deeltjes over elkaar, terwijl dit niet gebeurt als je alleen naar de externe dosis in de lucht kijkt. Deze dosismaat kan gebruikt worden om de effecten te voorspellen van zilver nanodeeltjes van een andere grootte dan in deze studie zijn getest. Voor het beschrijven van acute effecten is de totale oppervlakte van de zilver nanodeeltjes (in de alveoli) een geschikte dosismaat. Voor effecten op de lange termijn of chronische effecten kan de dosismaat anders zijn, omdat de kinetiek en het oplossen van de zilver nanodeeltjes dan een rol spelen in de verdeling van zilver over organen en weefsels van het lichaam, waar ze effecten kunnen veroorzaken die niet direct gerelateerd zijn aan de interne alveolaire dosis. Ook voor nanodeeltjes van een andere chemische samenstelling, vorm of coating, die verschillen van de zilver nanodeeltjes in deze studie, kan de geschikte dosismaat anders zijn door verschillen in kinetiek en in de mechanismen die toxiciteit veroorzaken.

\section{EVALUATIE VAN HET GEBRUIK VAN IN VITRO MODELLEN}

Dierstudies bieden unieke informatie over de verdeling en de effecten van nanomaterialen. Echter, het gebruik van dieren is vanuit ethisch oogpunt gezien niet wenselijk en moet zoveel mogelijk worden beperkt. Bovendien is het niet mogelijk om alle varianten van nanomaterialen in dieren te testen, gezien de enorme verscheidenheid aan nanomaterialen. Om het aantal dierstudies te beperken zijn verschillende in vitro modellen ontwikkeld die gebruikt kunnen worden voor het testen van nanomaterialen.

In hoofdstuk 5 wordt de voortgang en toekomst van in vitro longmodellen geëvalueerd om de translocatie (passage) van nanomaterialen over de long-bloed barrière te bestuderen. De afgelopen jaren zijn verschillende in vitro modellen ontwikkeld om de translocatie van nanomaterialen te bestuderen, omdat er zorgen zijn dat nanodeeltjes de barrières van het menselijk lichaam onbedoeld zouden kunnen passeren en zo het bloed en andere organen kunnen bereiken. Deze in vitro modellen variëren in complexiteit van een enkele laag cellen tot de combinatie van meerdere celtypen in een 3D model. Er zijn echter maar een paar studies beschikbaar die het mogelijk maken om de resultaten van in vitro modellen te vergelijken met data van dierstudies. Op dit moment kunnen in vitro modellen informatie geven over de relatieve mate van translocatie van nanodeeltjes. Het is nog niet mogelijk om de data van in vitro modellen te gebruiken om de exacte hoeveelheid nanodeeltjes, die de long-bloed barrière zou kunnen passeren in mensen, te voorspellen. Eenvoudige in vitro modellen hebben het voordeel dat ze gemakkelijk op een grote schaal toegepast kunnen worden, terwijl complexe modellen meer op de situatie in de mens lijken. De ontwikkelingen van meer complexe modellen die de menselijke situatie nog beter nabootsen, door co-culturen van verschillende celtypen en het gebruik van microfluidics, lijken veelbelovend. 
In hoofdstuk 6 wordt het gebruik van verschillende 'insert' membranen geëvalueerd om de translocatie van nanodeeltjes te bestuderen. 'Insert' membranen bestaan uit een plastic laag met poriën waar aan de ene zijde longcellen en aan de andere zijde bloedcellen gekweekt kunnen worden om zo de long-bloed barrière na te bootsen. De translocatie van fluorescente polystyreen nanodeeltjes over 'insert' membranen, die verschillen in porie-grootte en in membraanmateriaal, is bestudeerd. De translocatie was hoger in 'inserts' met grotere poriën vergeleken met kleinere poriën en hoger in polycarbonaat membranen vergeleken met polyester membranen. Fluorescente nanodeeltjes hebben als voordeel dat ze gemakkelijk te detecteren zijn tegen lage kosten. Echter, voor veel van de geteste concentraties was de translocatie van de nanodeeltjes lager dan de detectielimiet van het fluorescente signaal, zelfs wanneer de 'insert' membranen zonder cellen werden getest. Voordat translocatie van nanodeeltjes in vitro wordt getest, moet eerst worden gekeken of de nanodeeltjes de 'insert' membranen kunnen passeren. Daarnaast zijn gevoeliger methoden nodig om de translocatie te meten, zoals 'fluorescent correlation spectroscopie', 'ICP-Ms' of 'radio-labeling'.

In hoofdstuk 7 wordt aangetoond dat we dezelfde dosismaat voor zilver nanodeeltjes konden vinden als in de bovenstaande dierstudie, de totale oppervlakte van de deeltjes, in een in vitro model dat bestaat uit een enkele laag long epitheelcellen. Wanneer we het in vitro longmodel complexer maakten door er macrofagen aan toe te voegen en het model te kweken op 'insert' membranen, was de correlatie met de dierstudie lager. Een mogelijke verklaring dat de enkele laag long epitheelcellen overeenkomt met de resultaten van de dierstudie is dat er in vivo normaal gesproken een vast aantal macrofagen aanwezig is in de longen, ongeveer één macrofaag per alveoli (Gordon and Read, 2002, Kopf et al., 2015). Pas na een blootstelling worden meer macrofagen gerekruteerd. Wanneer deeltjes worden ingeademd en diep in de longen terecht komen, komen ze eerst in contact met long epitheelcellen die vervolgens allerlei stoffen produceren om macrofagen te rekruteren (Stone et al., 2007), maar eerst is er dus contact met de epitheelcellen. De macrofagen in het in vitro model nemen de nanodeeltjes op, waardoor ze de dosis die op de epitheelcellen komt verlagen en daarmee ook het effect. Voor het bestuderen van acute effecten van nanodeeltjes is het beter om een enkele laag epitheelcellen te gebruiken dan een co-cultuur met macrofagen. Voor effecten op de lange termijn zijn macrofagen wel belangrijk, omdat nanodeeltjes kunnen oplossen in lysosomen in macrofagen waardoor ionen vrij kunnen komen die de longen kunnen beschadigen. Ook kan de mogelijkheid van de longen om deeltjes op te ruimen worden aangetast wanneer er meer nanodeeltjes in de longen aanwezig zijn dan dat er door de macrofagen kan worden opgeruimd. Dit wordt ook wel 'lung particle overload' genoemd. Wanneer de effecten van nanomaterialen worden getest bij 'overload', dan zijn de macrofagen een essentieel onderdeel in het model.

In hoofdstuk 8 worden de resultaten van een studie gepresenteerd waarin eerder genoemd in vitro longmodel, gekweekt op 'insert' membranen, an zilver nanodeeltjes werd blootgesteld via de lucht. Het doel van deze studie was om te zien of de blootstelling en 
effecten van zilver nanodeeltjes via de lucht beter overeenkomen met de in vivo situatie dan na blootstelling via kweekmedium. Blootstelling van het longmodel zonder macrofagen aan zilver nanodeeltjes via de lucht veroorzaakte een dosis-afhankelijke toename in toxiciteit en ontstekingsparameters, net zoals in vivo, terwijl blootstelling via kweekmedium juist een afname in ontstekingsparameters veroorzaakte. Net zoals bij de eerdere experimenten in kweekmedium zorgde de toevoeging van macrofagen aan het longmodel voor een verlaging van de dosis van zilver nanodeeltjes op de epitheelcellen en dus voor bescherming. $\mathrm{Na}$ blootstelling via de lucht werden significante veranderingen in longtoxiciteit parameters gevonden bij doseringen tussen de $0.0025 \mathrm{en} 0.02 \mu \mathrm{g} / \mathrm{cm}^{2}$, die overlappen met de doseringen in vivo van 0.000 I tot $0.005 \mu \mathrm{g} / \mathrm{cm}^{2}$, terwijl er bij blootstelling via kweekmedium veel hogere doseringen tussen 3 en $30 \mu \mathrm{g} / \mathrm{cm}^{2}$ nodig waren om een significant effect te meten. Deze experimenten, waarin het longmodel werd blootgesteld aan zilver nanodeeltjes via de lucht, zijn slechts één keer uitgevoerd. De experimenten zullen in de toekomst herhaald moeten worden om te bevestigen dat blootstelling via de lucht beter overeenkomt met de in vivo situatie dan blootstelling via kweekmedium.

\section{IMPLEMENTATIE VAN DE GESCHIKTE DOSISMAAT}

In deze thesis hebben we de totale oppervlakte van zilver nanodeeltjes die de alveoli bereiken geïdentificeerd als een geschikte dosismaat voor het beschrijven van de dosis-response relatie tussen zilver nanodeeltjes en acute effecten na inademing. Deze dosismaat hebben we aangetoond in een dierstudie en in een in vitro longmodel dat bestond uit een enkele laag epitheelcellen. Er zijn meerdere verklaringen waarom de deeltjesoppervlakte van de deeltjes die de alveoli bereiken een geschikte dosismaat is: I) de alveoli is de plaats waar toxiciteit optreedt, 2) zilver nanodeeltjes van verschillende grootte hebben een verschillend depositie patroon in de longen, dus ook in de alveoli (Asgharian et al., 2009), 3) de grootte van de zilver nanodeeltjes heeft invloed op allerlei reacties in de cellen, zoals opname van deeltjes (Zhang et al., 20I4), 4) zilver ionen kunnen vrijkomen van het oppervlak van zilver nanodeeltjes (Ma et al., 20 I2, Leo et al., 20 I3, Stebounova et al., 20 I Ib, Kent and Vikesland, 20 I2, Zook et al., 20 I I) en deze ionen kunnen toxiciteit veroorzaken (Pratsinis et al., 20I3, Beer et al., 20I 2, Wang et al., 20I4b, Lubick, 2008, Park et al., 20I0), en 5) zilver nanodeeltjes kunnen toxiciteit veroorzaken door direct contact van hun oppervlak met de long epitheelcellen.

Deze dosismaat kan worden gebruikt in risicobeoordeling om de acute effecten te voorspellen van zilver nanodeeltjes van een grootte die niet eerder getest is. Bijvoorbeeld de productie van ontstekingsparameter MCP-I in de longen was verhoogd met $20 \%$ na blootstelling aan $1.7 \times 10^{9} \mathrm{~nm}^{2}$ zilver nanodeeltjes per $\mathrm{cm}^{2}$ van de alveoli, onafhankelijk van de deeltjesgrootte, terwijl de massa van de geteste deeltjes verschilde (0.000053 $\mu \mathrm{g} / \mathrm{cm}^{2}$ voor de $18 \mathrm{~nm}$ en $0.00010 \mu \mathrm{g} / \mathrm{cm}^{2}$ voor de $34 \mathrm{~nm}$ deeltjes). Als we nu zilver nanodeeltjes van $74 \mathrm{~nm}$ nemen, die niet eerder getest zijn, dan zal de productie van MCP-I waarschijnlijk ook $20 \%$ toenemen na blootstelling aan $1.7 \times 10^{9} \mathrm{~nm}^{2}$ zilver 
nanodeeltjes per $\mathrm{cm}^{2}$ van de alveoli, terwijl de dosis uitgedrukt in massa anders zal zijn vergeleken met de andere deeltjesgroottes $\left(0.00022 \mu \mathrm{g} / \mathrm{cm}^{2}\right)$. Voor de risicobeoordeling van nanodeeltjes met andere karakteristieken, zoals andere chemische samenstelling, coating of lading, zal de geschikte dosismaat nog bepaald moeten worden (in studies waarin nanodeeltjes worden getest die verschillen in één karakteristiek) om zo de effecten van andere nanomaterialen ook te kunnen voorspellen.

\section{TESTSTRATEGIE OM DE EFFECTEN VAN NANOMATERIALEN TE SCHATTEN}

Er is informatie nodig over zowel de blootstelling aan als ook over de effecten van nanomaterialen voor een juiste risicobeoordeling van nanomaterialen. De effecten van nanomaterialen kunnen worden onderzocht door het uitvoeren van in vivo en in vitro studies zoals beschreven in deze thesis. Omdat het gebruik van dieren niet wenselijk is en het ook niet mogelijk is om alle verschillende nanomaterialen in dieren te testen, is het belangrijk dat in vitro modellen worden gebruikt in de risicobeoordeling van nanomaterialen. Daarom wordt in hoofdstuk 9 een teststrategie gepresenteerd, waarin in vitro methoden een centrale rol spelen, om het gevaar van nanomaterialen na inademing te identificeren. De test strategie is bedoeld om de acute effecten te identificeren van nanomaterialen die nog niet (voldoende) zijn getest en van nieuw ontwikkelde nanomaterialen met als doel risico's te kunnen schatten zonder gebruik te maken van dierstudies. Zoals we al eerder beschreven zijn de acute effecten van nanomaterialen na inademing waarschijnlijk (gerelateerd aan) epitheelschade (hoofdstuk 7). Een enkele cellaag van epitheelcellen kan voldoende zijn om de dosismaat van verschillende nanomaterialen te bepalen en zo de acute effecten van nog meer nanomaterialen te voorspellen. Deze informatie kan vervolgens worden gebruikt in de risicobeoordeling van nanomaterialen.

\section{TOEKOMST PERSPECTIEVEN}

Informatie over de invloed van deeltjeskarakteristieken op het ontstaan van effecten na inademing kan worden gebruikt voor de ontwikkeling van Quantitative StructureActivity Relationships (QSARS) die de effecten van nieuwe nanomaterialen kunnen voorspellen. De data kunnen ook gecombineerd worden in een dataset waarin multivariate analyse uitgevoerd kan worden om zo het samenspel van verschillende deeltjeskarakteristieken te ontdekken die belangrijk zijn voor het ontstaan van effecten in de longen. Uiteindelijk kan informatie over (combinaties van) deeltjeskarakteristieken die gevaarlijk zijn, gebruikt worden om veilige nanomaterialen te ontwikkelen: safe-bydesign.

De strategie die we presenteerden in hoofdstuk 9 kan worden gebruikt in de risicobeoordeling van acute effecten van nanomaterialen na kortdurende inademing. In 
vitro modellen worden vooral gebruikt voor acute blootstellingen die meestal variëren tussen 4 uur en 24 uur. Er zijn echter zorgen dat een levenslange blootstelling aan een lage dosering nanomaterialen effecten kan veroorzaken die pas na jaren zichtbaar worden. De meeste studies (zowel in vivo als in vitro) focussen op acute effecten en kortdurende blootstelling aan nanomaterialen omdat langdurige studies kostbaar en tijdrovend zijn. Waarschijnlijk zijn de effecten van nanomaterialen na herhaaldelijke of langdurige blootstelling anders dan na acute blootstelling, omdat klaringsmechanismen en secundaire effecten een belangrijkere rol spelen. Daarom zal een enkele cellaag van epitheelcellen waarschijnlijk niet voldoende zijn om de effecten van nanomaterialen te bepalen na herhaaldelijke of langdurige blootstelling. Bovendien zijn in vitro modellen meestal gebaseerd op geïmmortaliseerde cellijnen die niet gebruikt kunnen worden voor herhaaldelijke of langdurige blootstelling, omdat ze blijven delen. Een oplossing voor dit probleem zou het gebruik van primaire cellen kunnen zijn, die voor langere tijd gekweekt kunnen worden. Primaire cellen hebben een fenotype dat meer kenmerken heeft die lijken op de cellen in het menselijk lichaam in vergelijking met cellijnen, maar de isolatie van primaire cellen is een uitdaging, ze verschillen van batch tot batch en ze kunnen hun kenmerken verliezen na verloop van tijd. Voor het uitvoeren van herhaaldelijke blootstellingen worden microfluidic systemen ontwikkeld die een mediumstroom gebruiken om de effecten van nanomaterialen te testen onder realistische omstandigheden. De continue mediumstroom brengt voedingsstoffen en neemt afvalstoffen mee en heeft invloed op de opname van nanomaterialen door cellen (Mahto et al., 2015). Daarnaast zorgt de continue mediumstroom voor een homogene blootstelling aan nanomaterialen, terwijl dit in statische systemen niet gebeurt. Voor effecten in de longen is een lung-on-a-chip ontwikkeld waarin mechanische krachten worden gebruikt om de bewegingen tijdens inademing na te bootsen waardoor de opname van nanomaterialen toenam (Huh et al., 20I0). De combinatie van deze microfluidics met primaire cellen is een veelbelovende methode om de effecten van nanomaterialen na herhaaldelijke of langdurige blootstelling te meten zonder gebruik te maken van dieren.

Samengevat, er is behoefte aan een adequate risicobeoordeling van nanomaterialen na inademing omdat er steeds meer nanomaterialen worden toegepast in consumenten producten. Het is niet mogelijk om binnen de enorme verscheidenheid aan nanomaterialen alle verschillende nanomaterialen apart te testen om zo de gevaren in te schatten. In deze thesis hebben wij de totale oppervlakte van zilver nanodeeltjes die de alveoli bereiken geïdentificeerd als de geschikte dosismaat om de acute effecten van zilver nanodeeltjes te beschrijven na zowel inademing door dieren als na blootstelling van een epitheel cellaag in vitro. Voor andere nanomaterialen, die verschillen in één karakteristiek, kan op dezelfde manier een geschikte dosismaat worden gevonden. Uiteindelijk kan hiervoor de epitheel cellaag gebruikt worden om dierstudies te verminderen. De geschikte dosismaat kan worden gebruikt om de effecten te voorspellen van nanomaterialen die niet eerder getest zijn. Daarnaast kan de informatie worden gebruikt om nanomaterialen te categoriseren op basis van hun 
gevaar voor de gezondheid. Dit kan worden vergeleken met de huidige blootstelling aan nanomaterialen door zowel werknemers als consumenten om zo de acute risico's voor de gezondheid in te schatten. Voor effecten na herhaaldelijke of langdurige blootstelling zullen in vitro systemen die meer op de mens lijken, zoals microfluidics, verder geoptimaliseerd moeten worden. 



\section{References}

(SCENIHR), S. C. O. E. A. N. I. H. R., DE JONG, W., DR., BRIDGES, J., PROF., DAWSON, K., PROF., JUNG, T., DR. \& PROYKOVA, A., PROF. 20Io. Scientific Basis for the Definition of the Term 'nanomaterial'. Brussels, Belgium: European Commission.

AARBIOU, J., ERTMANN, M., VAN WETERING, S., VAN NOORT, P., ROOK, D., RABE, K. F., LITVINOV, S. V., VAN KRIEKEN, J. H., DE BOER, W. I. \& HIEMSTRA, P. S. 2002. Human neutrophil defensins induce lung epithelial cell proliferation in vitro. J Leukoc Biol, 72, 167-74.

AL-HALLAK, M. H., SARFRAZ, M. K., AZARMI, S., ROA, W. H., FINLAY, W. H., ROULEAU, C. \& LOBENBERG, R. 20I2. Distribution of effervescent inhalable nanoparticles after pulmonary delivery: an in vivo study. Ther Deliv, 3, 725-34.

AL-SID-CHEIKH, M., ROULEAU, C. \& PELLETIER, E. 20I3. Tissue distribution and kinetics of dissolved and nanoparticulate silver in Iceland scallop (Chlamys islandica). Marine Environmental Research, 86, 2I-28.

ANJILVEL, S. \& ASGHARIAN, B. 1995. A multiple-path model of particle deposition in the rat lung. Fundam Appl Toxicol, 28, 4I-50.

ARAI, Y., MIYAYAMA, T. \& HIRANO, S. 20I4. Difference in the toxicity mechanism between ion and nanoparticle forms of silver in the mouse lung and in macrophages. Toxicology, 328c, 84-92. ARDAVIN, C., MARTINEZ DEL HOYO, G., MARTIN, P., ANJUERE, F., ARIAS, C. F., MARIN, A. R., RUIZ, S., PARRILLAS, V. \& HERNANDEZ, H. 200 I. Origin and differentiation of dendritic cells. Trends Immunol, 22, 691-700.

ARTS, J. H., MUIJSER, H., DUISTERMAAT, E., JUNKER, K. \& KUPER, C. F. 2007. Five-day inhalation toxicity study of three types of synthetic amorphous silicas in Wistar rats and post-exposure evaluations for up to 3 months. Food Chem Toxicol, 45, i 856-67.

ASATI, A., SANTRA, S., KAITTANIS, C. \& PEREZ, J. M. 2oro. Surface-charge-dependent cell localization and cytotoxicity of cerium oxide nanoparticles. ACS Nano, 4, 532 I-3 I.

ASGHARIAN, B., PRICE, O., MILLER, F., SUBRAMANIAM, R., CASSEE, F. R., FREIJER, J., VAN BREE, L. \& WINTER-SORKINA, R. 2009. Multiple-Path Particle Dosimetry Model (MPPD v 2.II): A Model for Human and Rat Airway Particle Dosimetry. In: APPLIED RESEARCH ASSOCIATES (ARA), H. I. F. H. S., NATIONAL INSTITUTE FOR PUBLIC HEALTH AND THE ENVIRONMENT (RIVM), AND MINISTRY OF HOUSING, SPATIAL PLANNING AND THE ENVIRONMENT (ed.) V2. I ed. Raleigh, North Carolina, USA: Applied Research Associates (ARA).

AUDI, G. \& WAPSTRA, A. H. I995. The I995 Update to the Atomic Mass Evaluation. Nucl Phys $A, 595,409-480$.

BAISCH, B. L., CORSON, N. M., WADE-MERCER, P., GELEIN, R., KENNELL, A. J., OBERDORSTER, G. \& ELDER, A. 20I4. Equivalent titanium dioxide nanoparticle deposition by intratracheal instillation and whole body inhalation: the effect of dose rate on acute respiratory tract inflammation. Part Fibre Toxicol, I I, I6.

BAKAND, S., HAYES, A. \& DECHSAKULTHORN, F. 20 г 2. Nanoparticles: a review of particle toxicology following inhalation exposure. Inhal Toxicol, 24, I 25-35.

BALASUBRAMANIAN, S. K., POH, K. W., ONG, C. N., KREYLING, W. G., ONG, W. Y. \& YU, L. E. 20I3. The effect of primary particle size on biodistribution of inhaled gold nano-agglomerates. Biomaterials, 34, 5439-52.

BARLOW, P. G., CLOUTER-BAKER, A., DONALDSON, K., MACCALLUM, J. \& STONE, V. 2005. Carbon black nanoparticles induce type II epithelial cells to release chemotaxins for alveolar macrophages. Part Fibre Toxicol, 2, I I. 
BARLOW, S., CHESSON, A., COLLINS, J. D., FLYNN, A., HARDY, A., JANY, K., KNAAP, A., KUIPER, H., LARSEN, J., LOVELL, D., LE NEINDRE, P., SCHANS, J., SCHLATTER, J., SILANO, V., SKERFVING, S. \& VANNIER, P. 2009. Use of the benchmark dose approach in risk assessment. The EFSA Journal, i I $50,72$.

BEDNAR, A. J., PODA, A. R., MITRANO, D. M., KENNEDY, A. J., GRAY, E. P., RANVILLE, J. F., HAYES, C. A., CROCKER, F. H. \& STEEVENS, J. A. 20I 3. Comparison of on-line detectors for field flow fractionation analysis of nanomaterials. Talanta, I04, I 40-8.

BEER, C., FOLDBJERG, R., HAYASHI, Y., SUTHERLAND, D. S. \& AUTRUP, H. 2012. Toxicity of silver nanoparticles - nanoparticle or silver ion? Toxicol Lett, 208, 286-92.

BHATIA, S. N. \& INGBER, D. E. 20I4. Microfluidic organs-on-chips. Nat Biotechnol, 32, 760-72. BLANK, F., ROTHEN-RUTISHAUSER, B. M., SCHURCH, S. \& GEHR, P. 2006. An optimized in vitro model of the respiratory tract wall to study particle cell interactions. J Aerosol Med, I9, 392405 .

BORM, P. J., ROBBINS, D., HAUBOLD, S., KUHLBUSCH, T., FISSAN, H., DONALDSON, K., SCHINS, R., STONE, V., KREYLING, W., LADEMANN, J., KRUTMANN, J., WARHEIT, D. \& OBERDORSTER, E. 2006. The potential risks of nanomaterials: a review carried out for ECETOC. Part Fibre Toxicol, 3, I I.

BOUWMEESTER, H., BRANDHOFF, P., MARVIN, H. J., WEIGEL, S. \& PETERS, R. J. 2014. State of the safety assessment and current use of nanomaterials in food and food production. Trends in Food Science and Technology, 40, 200-2 I0.

BRAAKHUIS, H. M., CASSEE, F. R., FOKKENS, P. H., DE IA FONTEYNE, L. J., OOMEN, A. G., KRYSTEK, P., DE JONG, W. H., VAN LOVEREN, H. \& PARK, M. V. 20 I sa. Identification of the appropriate dose metric for pulmonary inflammation of silver nanoparticles in an inhalation toxicity study. Nanotoxicology, I-I I.

BRAAKHUIS, H. M., GIANNAKOU, C., PEIJNENBURG, W. J. G. M., VERMEULEN, J., VAN LOVEREN, H. \& PARK, M. V. D. Z. 20 I 5 b. Simple in vitro models can predict pulmonary toxicity of silver nanoparticles. Nanotoxicology, accepted.

BRAAKHUIS, H. M., GOSENS, I., KRYSTEK, P., BOERE, J., CASSEE, F. R., FOKKENS, P., POST, J., VAN LOVEREN, H. \& PARK, M. 20I4a. Particle size dependent deposition and pulmonary inflammation after short-term inhalation of silver nanoparticles. Part Fibre Toxicol, I I, I6.

BRAAKHUIS, H. M., KLOET, S. K., KEZIC, S., KUPER, F., PARK, M. V., BELLMANN, S., VAN DER ZANDE, M., LE GAC, S., KRYSTEK, P., PETERS, R. J., RIETJENS, I. M. \& BOUWMEESTER, H. 20I5c. Progress and future of in vitro models to study translocation of nanoparticles. Arch Toxicol.

BRAAKHUIS, H. M., PARK, M. V., GOSENS, I., DE JONG, W. H. \& CASSEE, F. R. 2oi 4b. Physicochemical characteristics of nanomaterials that affect pulmonary inflammation. Part Fibre Toxicol, I I, I8.

BRANDENBERGER, C., MUHLFELD, C., Ali, Z., LENZ, A. G., SCHMID, O., PARAK, W. J., GEHR, P. \& ROTHEN-RUTISHAUSER, B. 2o I oa. Quantitative evaluation of cellular uptake and trafficking of plain and polyethylene glycol-coated gold nanoparticles. Small, 6, I669-78.

BRANDENBERGER, C., ROTHEN-RUTISHAUSER, B., MUHLFELD, C., SCHMID, O., FERRON, G. A., MAIER, K. L., GEHR, P. \& LENZ, A. G. 2oiob. Effects and uptake of gold nanoparticles deposited at the air-liquid interface of a human epithelial airway model. Toxicol Appl Pharmacol, 242, 56-65.

BRAUN, A., COUTEAU, O., FRANKS, K., KESTENS, V., ROEBBEN, G., LAMBERTY, A. \& LINSINGER, T. P. J. 20II. Validation of dynamic light scattering and centrifugal liquid sedimentation methods for nanoparticle characterisation. Advanced Powder Technology, 22, 766-770. BROOK, R. D., RAJAGOPALAN, S., POPE, C. A., 3 RD, BROOK, J. R., BHATNAGAR, A., DIEZ-ROUX, A. V., HOLGUIN, F., HONG, Y., LUEPKER, R. V., MITTLEMAN, M. A., PETERS, A., SISCOVICK, D., SMITH, S. C., JR., WHITSEL, L. \& KAUFMAN, J. D. 20 Iо. 
Particulate matter air pollution and cardiovascular disease: An update to the scientific statement from the American Heart Association. Circulation, I 2 I, 233 I-78.

BROWN, S. C., BOYKO, V., MEYERS, G., VOETZ, M. \& WOHLLEBEN, W. 2or 3. Toward advancing nano-object count metrology: a best practice framework. Environ Health Perspect, I I, I 282-9I.

BRUNAUER, S., EMMETT, P. H. \& TELLER, E. 1938. Absorption of gases in multimolecular layers. J Am Chem Soc, 60, 309-3I9.

CARLSON, C., HUSSAIN, S. M., SCHRAND, A. M., BRAYDICH-STOLLE, L. K., HESS, K. L., JONES, R. L. \& SCHLAGER, J. J. 2008. Unique cellular interaction of silver nanoparticles: sizedependent generation of reactive oxygen species. J Phys Chem B, I I 2, I3608-I9.

CARTWRIGHT, L., POULSEN, M. S., NIELSEN, H. M., POJANA, G., KNUDSEN, L. E., SAUNDERS, M. \& RYTTING, E. 20I2. In vitro placental model optimization for nanoparticle transport studies. Int J Nanomedicine, 7, 497-5 I0.

CARVALHO, T. C., PETERS, J. I. \& WILLIAMS, R. O., 3 RD 20 I I. Influence of particle size on regional lung deposition--what evidence is there? Int J Pharm, 406, I-Io.

CASCIO, C., GEISS, O., FRANCHINI, F., OJEA-JIMENEZ, I., ROSSI, F., GILLILAND, D. \& CALZOLAI, L. 20I 5 . Detection, quantification and derivation of number size distribution of silver nanoparticles in antimicrobial consumer products. Journal of Analytical Atomic Spectrometry.

CASSEE, F. R., MUIJSER, H., DUISTERMAAT, E., FREIJER, J. J., GEERSE, K. B., MARIJNISSEN, J. C. \& ARTS, J. H. 2002. Particle size-dependent total mass deposition in lungs determines inhalation toxicity of cadmium chloride aerosols in rats. Application of a multiple path dosimetry model. Arch Toxicol, 76, 277-86.

CASTRANOVA, V. 20I I. Overview of current toxicological knowledge of engineered nanoparticles. J Occup Environ Med, 53, Si 4-7.

CHANDLER, D. 2005. Interfaces and the driving force of hydrophobic assembly. Nature, 437, 640-7. CHENG, L. C., JIANG, X., WANG, J., CHEN, C. \& LIU, R. S. 20 I 3. Nano-bio effects: interaction of nanomaterials with cells. Nanoscale, 5, 3547-69.

CHIANEA, T., ASSIDJO, N. E. \& CARDOT, P. J. 2000. Sedimentation field-flow-fractionation: emergence of a new cell separation methodology. Talanta, 5 I, 835-47.

CHO, W. S., CHO, M., JEONG, J., CHOI, M., CHO, H. Y., HAN, B. S., KIM, S. H., KIM, H. O., LIM, Y. T., CHUNG, B. H. \& JEONG, J. 2009. Acute toxicity and pharmacokinetics of I 3 nm-sized PEG-coated gold nanoparticles. Toxicol Appl Pharmacol, 236, I 6-24.

CHO, W. S., DUFFIN, R., HOWIE, S. E., SCOTTON, C. J., WALLACE, W. A., MACNEE, W., BRADLEY, M., MEGSON, I. L. \& DONALDSON, K. 20I I. Progressive severe lung injury by zinc oxide nanoparticles; the role of $\mathrm{Zn} 2+$ dissolution inside lysosomes. Part Fibre Toxicol, 8, 27.

CHO, W. S., DUFFIN, R., POLAND, C. A., DUSCHL, A., OOSTINGH, G. J., MACNEE, W., BRADLEY, M., MEGSON, I. L. \& DONALDSON, K. 20I 2a. Differential pro-inflammatory effects of metal oxide nanoparticles and their soluble ions in vitro and in vivo; zinc and copper nanoparticles, but not their ions, recruit eosinophils to the lungs. Nanotoxicology, 6, 22-35.

CHO, W. S., DUFFIN, R., THIELBEER, F., BRADLEY, M., MEGSON, I. L., MACNEE, W., POLAND, C. A., TRAN, C. L. \& DONALDSON, K. 20 г 2b. Zeta potential and solubility to toxic ions as mechanisms of lung inflammation caused by metal/metal oxide nanoparticles. Toxicol Sci, I 26, 469-77.

CHOI, H. S., ASHITATE, Y., LEE, J. H., KIM, S. H., MATSUI, A., INSIN, N., BAWENDI, M. G., SEMMLER-BEHNKE, M., FRANGIONI, J. V. \& TSUDA, A. 201 . Rapid translocation of nanoparticles from the lung airspaces to the body. Nat Biotechnol, 28, I300-3.

CHOI, H. S., LIU, W., MISRA, P., TANAKA, E., ZIMMER, J. P., ITTY IPE, B., BAWENDI, M. G. \& FRANGIONI, J. V. 2007. Renal clearance of quantum dots. Nat Biotechnol, 25, i i 65-70. CHOWDHURY, F., HOWAT, W. J., PHILlIPS, G. J. \& LACKIE, P. M. 20io. Interactions between endothelial cells and epithelial cells in a combined cell model of airway mucosa: effects on tight junction permeability. Exp Lung Res, 36, I-I I. 
CHRISTENSEN, F. M., JOHNSTON, H. J., STONE, V., AITKEN, R. J., HANKIN, S., PETERS, S. \& ASCHBERGER, K. 20I0. Nano-silver - feasibility and challenges for human health risk assessment based on open literature. Nanotoxicology, 4, 284-95.

COIN, P. G., ROGGLI, V. L. \& BRODY, A. R. I 994. Persistence of long, thin chrysotile asbestos fibers in the lungs of rats. Environ Health Perspect, I02 Suppl 5, 197-9.

COLOGNATO, R., PARK, M. V. D. Z., WICK, P. \& DE JONG, W. H. 20 I 2. Interactions with the Human Body. In: FADEEL, B., PIETROIUSTI, A. \& SHVEDOVA, A. A. (eds.) Adverse Effects of Engineered Nanomaterials: Exposure, Toxicology and Impact on Human Health. Amsterdam: Elsevier. COMMISSION, E. 20I I. Commission Recommendation of I 8 October 20 I I on the definition of nanomaterial. In: COMMISSION, E. (ed.) 2011/696/EU. Official Journal of the European Union. COMOUTH, A., SAATHOFF, H., NAUMANN, K., MUELHOPT, S., PAUR, H. R. \& LEISNER, T. 20I3. Modelling and measurement of particle deposition for cell exposure at the airliquid interface. Journal of Aerosol Science, 63, I 2.

CREUTZENBERG, O., BELLMANN, B., KOROLEWITZ, R., KOCH, W., MANGELSDORF, I., TILLMANN, T. \& SCHAUDIEN, D. 20I 2. Change in agglomeration status and toxicokinetic fate of various nanoparticles in vivo following lung exposure in rats. Inhal Toxicol, 24, 82 I-30.

DE BOER, J. L., RITSEMA, R., PISO, S., VAN STADEN, H. \& VAN DEN BELD, W. 2004. Practical and quality-control aspects of multi-element analysis with quadrupole iCp-ms with special attention to urine and whole blood. Anal Bioanal Chem, 379, 872-80.

DE JONG, W. H., BURGER, M. C., VERHEIJEN, M. A. \& GEERTSMA, R. E. 2o Io. Detection of the Presence of Gold Nanoparticles in Organs by Transmission Electron Microscopy. Materials, 3, 468I-4694.

DE JONG, W. H., HAGENS, W. I., KRYSTEK, P., BURGER, M. C., SIPS, A. J. \& GEERTSMA, R. E. 2008. Particle size-dependent organ distribution of gold nanoparticles after intravenous administration. Biomaterials, 29, I91 2-9.

DEGUELDRE, C., FAVARGER, P. Y., ROSSE, R. \& WOLD, S. 2006a. Uranium colloid analysis by single particle inductively coupled plasma-mass spectrometry. Talanta, 68, 623-8.

DEGUELDRE, C., FAVARGER, P. Y. \& WOLD, S. 2006b. Gold Colloid Analysis by Inductively Coupled Plasma-mass Spectrometry in a Single Particle Mode. Anal Chim Acta, 555, 263-268.

DELMAAR, C. J., PEIJNENBURG, W. J., OOMEN, A. G., CHEN, J., DE JONG, W. H., SIPS, A. J., WANG, Z. \& PARK, M. V. 2015. A practical approach to determine dose metrics for nanomaterials. Environ Toxicol Chem, 34, I0 1 5-22.

DELOID, G., COHEN, J. M., DARRAH, T., DERK, R., ROJANASAKUL, L., PYRGIOTAKIS, G., WOHLLEBEN, W. \& DEMOKRITOU, P. 20I4. Estimating the effective density of engineered nanomaterials for in vitro dosimetry. Nat Commun, 5, 35 I4.

DIABATE, S., MULHOPT, S., PAUR, H. R. \& KRUG, H. F. 2008. The response of a co-culture lung model to fine and ultrafine particles of incinerator fly ash at the air-liquid interface. Altern Lab Anim, 36, 285-98.

DICK, C. A., SINGH, P., DANIELS, M., EVANSKY, P., BECKER, S. \& GILMOUR, M. I. 2003. Murine pulmonary inflammatory responses following instillation of size-fractionated ambient particulate matter. J Toxicol Environ Health A, 66, 2193-2207.

DONALDSON, K. \& BORM, P. J. 2006. Interactions between macrophages and epithelial cells. In: PRESS, C. (ed.) Particle Toxicology.

DONALDSON, K., MURPHY, F. A., DUFFIN, R. \& POLAND, C. A. 2oroa. Asbestos, carbon nanotubes and the pleural mesothelium: a review of the hypothesis regarding the role of long fibre retention in the parietal pleura, inflammation and mesothelioma. Part Fibre Toxicol, 7, 5.

DONALDSON, K. \& POLAND, C. A. 20I 2. Inhaled nanoparticles and lung cancer - what we can learn from conventional particle toxicology. Swiss Med Wkly, I 42, w 3547.

DONALDSON, K., POLAND, C. A. \& SCHINS, R. P. 2orob. Possible genotoxic mechanisms of nanoparticles: criteria for improved test strategies. Nanotoxicology, 4, 4I 4-20.

DONALDSON, K., SCHINWALD, A., MURPHY, F., CHO, W. S., DUFFIN, R., TRAN, L. \& 
POLAND, C. 20 r 2. The Biologically Effective Dose in Inhalation Nanotoxicology. Acc Chem Res. DONALDSON, K., STONE, V., CLOUTER, A., RENWICK, L. \& MACNEE, W. 200 I. Ultrafine particles. Occup Environ Med, 58, 2 I I-6, I 99.

DOS SANTOS, T., VARELA, J., LYNCH, I., SALVATI, A. \& DAWSON, K. A. 20 I I. Effects of transport inhibitors on the cellular uptake of carboxylated polystyrene nanoparticles in different cell lines. PLoS One, 6, e24438.

DU, Y. K., YANG, P., MOU, Z. G., HUA, N. P. \& JIANG, L. 2006. Thermal Decomposition Behaviors of PvP coated on Platinum Nanoparticles. Journal of Applied Polymer Science, 99, 4.

DUDKIEWICZ, A., TIEDE, K., LOESCHNER, K., JENSEN, L. H. S., JENSEN, E., WIERZBICKI, R., BOXALL, A. B. A. \& MOLHAVE, K. 20I I. Characterization of nanomaterials in foor by electronmicroscopy. Trends in Analytical Chemistry, 30, 28-43.

DUFFIN, R., TRAN, L., BROWN, D., STONE, V. \& DONALDSON, K. 2007. Proinflammogenic effects of low-toxicity and metal nanoparticles in vivo and in vitro: highlighting the role of particle surface area and surface reactivity. Inhal Toxicol, I9, 849-56.

EFSA 2009. Guidance of the Scientific Committe on a request from EFSA on the use of the benchmark dose approach in risk assessment. The EFSA Journal, I I 50, I-72.

EFSA 20I I. Scientific Opinion on Guidance on the risk assessment of the application of nanoscience and nanotechnologies in the food and feed chain. The EFSA Journal, 9, 36.

ELDER, A., GELEIN, R., SILVA, V., FEIKERT, T., OPANASHUK, L., CARTER, J., POTTER, R., MAYNARD, A., ITO, Y., FINKELSTEIN, J. \& OBERDORSTER, G. 2006. Translocation of inhaled ultrafine manganese oxide particles to the central nervous system. Environ Health Perspect, II 4 , II $72-8$.

ELSAESSER, A. \& HOWARD, C. V. 20 i 2. Toxicology of nanoparticles. Adv Drug Deliv Rev, 64, I29-37.

ELZEY, S., TSAI, D. H., RABB, S. A., YU, L. L., WINCHESTER, M. R. \& HACKLEY, V. A. 20I2. Quantification of ligand packing density on gold nanoparticles using iCp-oes. Anal Bioanal Chem, 403, I 45-9.

FAZLOLLAHI, F., SIPOS, A., KIM, Y. H., HAMM-ALVAREZ, S. F., BOROK, Z., KIM, K. J. \& CRANDALL, E. D. 20 I I. Translocation of PEGylated quantum dots across rat alveolar epithelial cell monolayers. Int J Nanomedicine, 6, 2849-57.

FILIPE, V., HAWE, A. \& JISKOOT, W. 20го. Critical evaluation of Nanoparticle Tracking Analysis (ntA) by NanoSight for the measurement of nanoparticles and protein aggregates. Pharm Res, 27, 796-8 I0.

FRANEK, J. 200 I. Temperature Dependence of Silver Oxide Formation. In: MINNESOTA, U. O. (ed.). Minnesota.

FRIEKE KUPER, C., GROLLERS-MULDERIJ, M., MAARSCHALKERWEERD, T., MEULENDIJKS, N. M., REUS, A., VAN ACKER, F., ZONDERVAN-VAN DEN BEUKEN, E. K., WOUTERS, M. E., BIJLSMA, S. \& KOOTER, I. M. 201 5. Toxicity assessment of aggregated/ agglomerated cerium oxide nanoparticles in an in vitro $3 \mathrm{D}$ airway model: the influence of mucociliary clearance. Toxicol In Vitro, 29, 389-97.

FROHLICH, E., BONSTINGL, G., HOFLER, A., MEINDL, C., LEITINGER, G., PIEBER, T. R. $\&$ ROBLEGG, E. 2013. Comparison of two in vitro systems to assess cellular effects of nanoparticlescontaining aerosols. Toxicol In Vitro, 27, 409-I 7.

GEISER, M. 20Iо. Update on macrophage clearance of inhaled micro- and nanoparticles. $J$ Aerosol Med Pulm Drug Deliv, 23, 207-I7.

GEISER, M., CASAULTA, M., KUPFERSCHMID, B., SCHULZ, H., SEMMLER-BEHNKE, M. \& KREYLING, W. 2008. The role of macrophages in the clearance of inhaled ultrafine titanium dioxide particles. Am J Respir Cell Mol Biol, 38, 37 I-6.

GEISER, M. \& KREYLING, W. G. 20I0. Deposition and biokinetics of inhaled nanoparticles. Part Fibre Toxicol, 7, 2.

GEISER, M., ROTHEN-RUTISHAUSER, B., KAPP, N., SCHURCH, S., KREYLING, W., 
SCHULZ, H., SEMMLER, M., IM HOF, V., HEYDER, J. \& GEHR, P. 2005. Ultrafine particles cross cellular membranes by nonphagocytic mechanisms in lungs and in cultured cells. Environ Health Perspect, I I3, I 555-60.

GENSCHOW, E., SPIELMANN, H., SCHOLZ, G., SEILER, A., BROWN, N., PIERSMA, A., BRADY, M., CLEMANN, N., HUUSKONEN, H., PAILLARD, F., BREMER, S. \& BECKER, K. 2002. The ECVAM international validation study on in vitro embryotoxicity tests: results of the definitive phase and evaluation of prediction models. European Centre for the Validation of Alternative Methods. Altern Lab Anim, 30, I 5 I-76.

GEORGE, I., VRANIC, S., BOLAND, S., COURTOIS, A. \& BAEZA-SQUIBAN, A. 2015. Development of an in vitro model of human bronchial epithelial barrier to study nanoparticle translocation. Toxicol In Vitro, 29, 5 I-8.

GERAETS, L., OOMEN, A. G., KRYSTEK, P., JACOBSEN, N. R., WALLIN, H., LAURENTIE, M., VERHAREN, H. W., BRANDON, E. F. \& DE JONG, W. H. 20I4. Tissue distribution and elimination after oral and intravenous administration of different titanium dioxide nanoparticles in rats. Part Fibre Toxicol, i I, 30.

GERAETS, L., OOMEN, A. G., SCHROETER, J. D., COLEMAN, V. A. \& CASSEE, F. R. 20 I 2. Tissue Distribution of Inhaled Micro- and Nano-sized Cerium Oxide Particles in Rats: Results From a 28-Day Exposure Study. Toxicol Sci, I 27, 463-73.

GERLOFS-NIJLAND, M. E., DORMANS, J. A., BLOEMEN, H. J., LESEMAN, D. L. \& BOERE, J. A. F. 2007. Toxicity of coarse and fine particulate matter from sites with contrasting traffic profiles. Inhal Toxicol, 19, I055-1069.

GESSNER, A., LIESKE, A., PAULKE, B. \& MULLER, R. 2002. Influence of surface charge density on protein adsorption on polymeric nanoparticles: analysis by two-dimensional electrophoresis. Eur J Pharm Biopharm, 54, I65-70.

GEYS, J., COENEGRACHTS, L., VERCAMMEN, J., ENGELBORGHS, Y., NEMMAR, A., NEMERY, B. \& HOET, P. H. 2006. In vitro study of the pulmonary translocation of nanoparticles: a preliminary study. Toxicol Lett, I60, 21 8-26.

GEYS, J., DE VOS, R., NEMERY, B. \& HOET, P. H. 2009. In vitro translocation of quantum dots and influence of oxidative stress. Am J Physiol Lung Cell Mol Physiol, 297, L903-I I.

GIBSON, N., HOLZWARTH, U., ABBAS, K., SIMONELLI, F., KOZEMPEL, J., CYDZIK, I., COTOGNO, G., BUlGHERONI, A., GILlilAND, D., PONTI, J., FRANCHINI, F., MARMORATO, P., STAMM, H., KREYLING, W., WENK, A., SEMMLER-BEHNKE, M., BUONO, S., MACIOCCO, L. \& BURGIO, N. 20I I. Radiolabelling of engineered nanoparticles for in vitro and in vivo tracing applications using cyclotron accelerators. Arch Toxicol, 85, 75 I-73. GIDDINGS, J. C. I973. The Conceptual Basis of Field-flow Fractionation. J Chem Educ, 50, 667. GLIGA, A. R., SKOGLUND, S., WALLINDER, I. O., FADEEL, B. \& KARLSSON, H. L. 2014. Size-dependent cytotoxicity of silver nanoparticles in human lung cells: the role of cellular uptake, agglomeration and Ag release. Part Fibre Toxicol, i I, I I.

GORDON, S. B. \& READ, R. C. 2002. Macrophage defences against respiratory tract infections. Br Med Bull, 6I, 45-6I.

GOSENS, I., MATHIJSSEN, L. E., BOKKERS, B. G., MUIJSER, H. \& CASSEE, F. R. 20 I 3. Comparative hazard identification of nano- and micro-sized cerium oxide particles based on 28-day inhalation studies in rats. Nanotoxicology.

GOSENS, I., MATHIJSSEN, L. E., BOKKERS, B. G., MUIJSER, H. \& CASSEE, F. R. 2014. Comparative hazard identification of nano- and micro-sized cerium oxide particles based on 28-day inhalation studies in rats. Nanotoxicology, 8, 643-53.

GOSENS, I., POST, J. A., DE lA FONTEYNE, L. J., JANSEN, E. H., GEUS, J. W., CASSEE, F. R. \& DE JONG, W. H. 20Iо. Impact of agglomeration state of nano- and submicron sized gold particles on pulmonary inflammation. Part Fibre Toxicol, 7, 37.

GRASSIAN, V. H., O'SHAUGHNESSY P, T., ADAMCAKOVA-DODD, A., PETTIBONE, J. M. \& THORNE, P. S. 2007. Inhalation exposure study of titanium dioxide nanoparticles with a primary particle size of 2 to $5 \mathrm{~nm}$. Environ Health Perspect, I I 5 , 397-402. 
GRIEP, L. M., WOLBERS, F., DE WAGENAAR, B., TER BRAAK, P. M., WEKSLER, B. B., ROMERO, I. A., COURAUD, P. O., VERMES, I., VAN DER MEER, A. D. \& VAN DEN BERG, A. 20I3. BBB on chip: microfluidic platform to mechanically and biochemically modulate bloodbrain barrier function. Biomed Microdevices, I 5, I 45-50.

GROGER, D., PAULUS, F., LICHA, K., WELKER, P., WEINHART, M., HOLZHAUSEN, C., MUNDHENK, L., GRUBER, A. D., ABRAM, U. \& HAAG, R. 20I3. Synthesis and biological evaluation of radio and dye labeled amino functionalized dendritic polyglycerol sulfates as multivalent anti-inflammatory compounds. Bioconjug Chem, 24, I 507-I 4.

GSCHWIND, S., FLAMIGNI, L., KOCH, J., BOROVINSKAYA, O., GROH, S., NIEMAX, K. \& GUNTHER, D. 20I I. Capabilities of Inductively Couples Plasma Mass Spectrometry for the Detection of Nanoparticles Carried by Monodisperse Microdroplets. J Anal At Spectrom, 26, i I66I 774 .

HAN, X., CORSON, N., WADE-MERCER, P., GELEIN, R., JIANG, J., SAHU, M., BISWAS, P., FINKELSTEIN, J. N., ELDER, A. \& OBERDORSTER, G. 20 I 2 . Assessing the relevance of in vitro studies in nanotoxicology by examining correlations between in vitro and in vivo data. Toxicology, 297, I-9.

HARINGTON, J. S., MACNAB, G. M., MILLER, K. \& KING, P. C. i97I. Enhancement of haemolytic activity of asbestos by heat-labile factors in fresh serum. Med Lav, 62, I7 I-6.

HARINK, B., LE GAC, S., TRUCKENMULLER, R., VAN BLITTERSWIJK, C. \& HABIBOVIC, P. 2013. Regeneration-on-a-chip? The perspectives on use of microfluidics in regenerative medicine. Lab Chip, I3, 35 I 2-28.

HARTUNG, T., BALLS, M., BARDOUILLE, C., BLANCK, O., COECKE, S., GSTRAUNTHALER, G., LEWIS, D. \& FORCE, E. G. C. C. P. T. 2002. Good Cell Culture Practice. ECVAM Good Cell Culture Practice Task Force Report I. Altern Lab Anim, 30, 407-I 4.

HARTUNG, T., LUECHTEFELD, T., MAERTENS, A. \& KLEENSANG, A. 2013. Integrated testing strategies for safety assessments. ALTEX, 30, 3-1 8.

HASLETON, P. S. I972. The internal surface area of the adult human lung. $J$ Anat, i I 2, 391-400. HASSELLOV, M., READMAN, J. W., RANVILLE, J. F. \& TIEDE, K. 2008. Nanoparticle analysis and characterization methodologies in environmental risk assessment of engineered nanoparticles. Ecotoxicology, 17, 344-61.

HEINRICH, U., FUHST, R., RITTINGHAUSEN, S., CREUTZENBERG, O., BELLMANN, B., KOCH, W. \& LEVSEN, K. I995. Chronic inhalation exposure of Wistar rats and two different strains of mice to diesel exhaust, carbon black and titanium dioxide. Inhal Toxicol, 7, 23.

HERAUlT, J., HAVARD, T. \& GOENAGE-INFANTE, H. 20 2. Asymmetric Flow Field Flow Fractionation Coupled with iCp-ms for Speciation Analysis of plasma Proteins. The Column, 8, I II 4 .

HERMANNS, M. I., KASPER, J., DUBRUEL, P., POHL, C., UBOLDI, C., VERMEERSCH, V., FUCHS, S., UNGER, R. E. \& KIRKPATRICK, C. J. 20 Iо. An impaired alveolar-capillary barrier in vitro: effect of proinflammatory cytokines and consequences on nanocarrier interaction. $J R$ Soc Interface, 7 Suppl I, S4I-54.

HERMANNS, M. I., UNGER, R. E., KEHE, K., PETERS, K. \& KIRKPATRICK, C. J. 2004. Lung epithelial cell lines in coculture with human pulmonary microvascular endothelial cells: development of an alveolo-capillary barrier in vitro. Lab Invest, 84, 736-52.

HERZOG, F., CLIFT, M. J., PICCAPIETRA, F., BEHRA, R., SCHMID, O., PETRI-FINK, A. \& ROTHEN-RUTISHAUSER, B. 2013. Exposure of silver-nanoparticles and silver-ions to lung cells in vitro at the air-liquid interface. Part Fibre Toxicol, ı,, I I.

HINDS, W. C. I982. Chapter I2. Aerosol Technology: Properties, Behavior and Measurement of Airborne Particles. New York: John Wiley \& Sons.

HO, M., WU, K. Y., CHEIN, H. M., CHEN, L. C. \& CHENG, T. J. 2o I I. Pulmonary toxicity of inhaled nanoscale and fine zinc oxide particles: mass and surface area as an exposure metric. Inhal Toxicol, 23, 947-56. 
HOLDER, A. L. \& MARR, L. C. 2013. Toxicity of silver nanoparticles at the air-liquid interface. Biomed Res Int, 20I 3, 328934.

HOLZHAUSEN, C., GRÖGER, D., MUNDHENK, L., WELKER, P., HAAG, R. \& GRUBER, A. D. 2013. Tissue and cellular localization of nanoparticles using $35 \mathrm{~S}$ labeling and light microscopic autoradiography. Nanomedicine: Nanotechnology, Biology and Medicine, 9, 465-468.

HORIE, M., FUKUI, H., ENDOH, S., MARU, J., MIYAUCHI, A., SHICHIRI, M., FUJITA, K., NIKI, E., HAGIHARA, Y., YOSHIDA, Y., MORIMOTO, Y. \& IWAHASHI, H. 20 I 2. Comparison of acute oxidative stress on rat lung induced by nano and fine-scale, soluble and insoluble metal oxide particles: $\mathrm{NiO}$ and $\mathrm{TiO}_{2}$. Inhal Toxicol, 24, 39I-400.

HORNUNG, V., BAUERNFEIND, F., HALLE, A., SAMSTAD, E. O., KONO, H., ROCK, K. L., FITZGERALD, K. A. \& LATZ, E. 2008. Silica crystals and aluminum salts activate the NALP 3 inflammasome through phagosomal destabilization. Nat Immunol, 9, 847-56.

HSIAO, I. L. \& HUANG, Y. J. 20II. Effects of various physicochemical characteristics on the toxicities of $\mathrm{ZnO}$ and $\mathrm{TiO}$ nanoparticles toward human lung epithelial cells. Sci Total Environ, 409, I $219-28$.

HUDA, S., SMOUKOV, S. K., NAKANISHI, H., KOWALCZYK, B., BISHOP, K. \& GRZYBOWSKI, B. A. 20I0. Antibacterial nanoparticle monolayers prepared on chemically inert surfaces by cooperative electrostatic adsorption (CELA). ACS Appl Mater Interfaces, 2, I 206- Io.

HUH, D., FUJIOKA, H., TUNG, Y. C., FUTAI, N., PAINE, R., 3 RD, GROTBERG, J. B. \& TAKAYAMA, S. 2007. Acoustically detectable cellular-level lung injury induced by fluid mechanical stresses in microfluidic airway systems. Proc Natl Acad Sci US A, I04, I8886-9I.

HUH, D., KIM, H. J., FRASER, J. P., SHEA, D. E., KHAN, M., BAHINSKI, A., HAMILTON, G. A. \& INGBER, D. E. 20I 3. Microfabrication of human organs-on-chips. Nat Protoc, 8, 2 I 35-57. HUH, D., MATTHEWS, B. D., MAMMOTO, A., MONTOYA-ZAVALA, M., HSIN, H. Y. \& INGBER, D. E. 20Io. Reconstituting organ-level lung functions on a chip. Science, 328, I662-8.

HUH, D., TORISAWA, Y. S., HAMILTON, G. A., KIM, H. J. \& INGBER, D. E. 20 I 2. Microengineered physiological biomimicry: organs-on-chips. Lab Chip, I2, 2 I 56-64.

HUK, A., IZAK-NAU, E., REIDY, B., BOYLES, M., DUSCHL, A., LYNCH, I. \& DUSINSKA, M. 20 I 4. Is the toxic potential of nanosilver dependent on its size? Part Fibre Toxicol, I I, 65.

HUSSAIN, N., JAITLEY, V. \& FLORENCE, A. T. 200 I. Recent advances in the understanding of uptake of microparticulates across the gastrointestinal lymphatics. Advanced Drug Delivery Reviews, 50, I07-I 42.

HUSSAIN, S., BOLAND, S., BAEZA-SQUIBAN, A., HAMEL, R., THOMASSEN, L. C., MARTENS, J. A., BILLON-GALLAND, M. A., FLEURY-FEITH, J., MOISAN, F., PAIRON, J. C. \& MARANO, F. 2009. Oxidative stress and proinflammatory effects of carbon black and titanium dioxide nanoparticles: role of particle surface area and internalized amount. Toxicology, 260, I 42-9. iCrp 1994. Human respiratory tract model for radiological protection. iCrp Publication 66. International Commission on Radiological Protection.

ILSI 2000. The relevance of the rat lung response to particle overload for human risk assessment: a workshop consensus report. ILSI Risk Science Institute Workshop Participants. Inhal Toxicol, I2, I-I 7 .

JAKUBOWSKI, N., MOENS, L. \& VANHAECKE, F. 1998. Sector-field Mass Spectrometers in ICPMS. Spectrochim Acta, Part B: Atom Spectrom, 53, I739-I763.

JENSEN, K. A., KEMBOUCHE, Y., CHRISTIANSEN, E., JACOBSON, N. R., WALLIN, H., GUIOT, C., SPALLA, O. \& WITSCHGER, O. 20 I I. Final protocol for producting suitable manufactures nanomaterial exposure media. In: NANOGENOTOX (ed.).

JI, J. H., JUNG, J. H., KIM, S. S., YOON, J. U., PARK, J. D., CHOI, B. S., CHUNG, Y. H., KWON, I. H., JEONG, J., HAN, B. S., SHIN, J. H., SUNG, J. H., SONG, K. S. \& YU, I. J. 2007. Twenty-eight-day inhalation toxicity study of silver nanoparticles in Sprague-Dawley rats. Inhal Toxicol, I9, 857-7 I.

KANDAROVA, H. \& LETASIOVA, S. 20 I I. Alternative methods in toxicology: pre-validated and validated methods. Interdiscip Toxicol, 4, I07-I 3. 
KENNEL, S. J., WOODWARD, J. D., RONDINONE, A. J., WALL, J., HUANG, Y. \& MIRZADEH, S. 2008. The fate of MAb-targeted Cdi25mTe/ZnS nanoparticles in vivo. Nuclear Medicine and Biology, 35, 50 I-5 I4.

KENT, R. D. \& VIKESLAND, P. J. 20 I 2. Controlled evaluation of silver nanoparticle dissolution using atomic force microscopy. Environ Sci Technol, 46, 6977-84.

KHLEBTSOV, N. \& DYKMAN, L. 20I . Biodistribution and toxicity of engineered gold nanoparticles: a review of in vitro and in vivo studies. Chem Soc Rev, 40, I647-7I.

KHLYSTOV, A., STANIER, C. \& PANDIS, S. N. 2004. An Algorithm for Combining Electrical Mobility and Aerodynamic Size Distributions Data when Measuring Ambient Aerosol. Aerosol Science and Technology, 229-238.

KIM, H. J. \& INGBER, D. E. 20I3. Gut-on-a-Chip microenvironment induces human intestinal cells to undergo villus differentiation. Integr Biol (Camb), 5, I I 30-40.

KIRKLAND, J. J., YAU, W. W., DOERNER, W. A. \& GRANT, J. W. I980. Sedimentation Field Flow Fractionation of Macro-molecules and Colloids. Anal Chem, 52, I 944-I 954.

KLEIN, S. G., HENNEN, J., SERCHI, T., BLOMEKE, B. \& GUTLEB, A. C. 20 i r. Potential of coculture in vitro models to study inflammatory and sensitizing effects of particles on the lung. Toxicol In Vitro, 25, I 5 16-34.

KLEIN, S. G., SERCHI, T., HOFFMANN, L., BLÖMEKE, B. \& GUTLEB, A. C. 2or3. An improved ${ }_{3} \mathrm{D}$ tetraculture system mimicking the cellular organisation at the alveolar barrier to study the potential toxic effects of particles on the lung. Particle and Fibre Toxicology.

KNAAPEN, A. M., BORM, P. J., ALBRECHT, C. \& SCHINS, R. P. 2004. Inhaled particles and lung cancer. Part A: Mechanisms. Int J Cancer, I09, 799-809.

KOBAYASHI, N., NAYA, M., ENDOH, S., MARU, J., YAMAMOTO, K. \& NAKANISHI, J. 2009. Comparative pulmonary toxicity study of nano- $\mathrm{TiO}(2)$ particles of different sizes and agglomerations in rats: different short- and long-term post-instillation results. Toxicology, 264, I I o-8. KOCH, W. \& STOBER, W. 200I. A simple pulmonary retention model accounting for dissolution and macrophage-mediated removal of deposited polydisperse particles. Inhal Toxicol, I 3, I 29-48.

KOPF, M., SCHNEIDER, C. \& NOBS, S. P. 2015 . The development and function of lung-resident macrophages and dendritic cells. Nat Immunol, I6, 36-44.

KREYLING, W. G. I 992. Intracellular particle dissolution in alveolar macrophages. Environ Health Perspect, 97, I 2 I-6.

KREYLING, W. G. 20I3. RE: Coagulation kinetics of $30 \mathrm{~nm}$ nanoparticles aerosol.

KREYLING, W. G., SEMMLER-BEHNKE, M., SEITZ, J., SCYMCZAK, W., WENK, A., MAYER, P., TAKENAKA, S. \& OBERDORSTER, G. 2009. Size dependence of the translocation of inhaled iridium and carbon nanoparticle aggregates from the lung of rats to the blood and secondary target organs. Inhal Toxicol, 2 I Suppl I, 55-60.

KREYLING, W. G., SEMMLER, M., ERBE, F., MAYER, P., TAKENAKA, S., SCHULZ, H., OBERDORSTER, G. \& ZIESENIS, A. 2002. Translocation of ultrafine insoluble iridium particles from lung epithelium to extrapulmonary organs is size dependent but very low. J Toxicol Environ Health A, 65, I 513-30.

KRYSTEK, P. 20I2. A review on approaches to biodistribution studies about gold and silver engineered nanoparticles by inductively couples plasma mass spectrometry. Microchemical Journal, I05, 39-43.

KRYSTEK, P., BRAAKHUIS, H. M., PARK, M. V. D. Z. \& JONG, W. H. D. 20I3. Inductively Coupled Plasma-Mass Spectrometry in Biodistribution Studies of (Engineered) Nanoparticles. Encyclopedia of Analytical Chemistry. I 5 March 2013 ed.: John Wiley \& Sons, Ltd.

KRYSTEK, P. \& DE JONG, W. H. 20 I 2.

KRYSTEK, P., JOCKS, T. \& ROESSNER, D. 201 2. Characterization of Au Nanoparticles in a Complex Biological Matrix [Online]. Available: http://www.wyatt.com/files/literature/Field_Flow_ Fractionation_A4F_Characterization_of_Au_Nanoparticles.pdf [Accessed 20 I 2-I0-I 8.

KRYSTEK, P., KETTLER, K., VAN DER WAGT, B. \& DE JONG, W. H. 2015. Exploring 
influences on the cellular uptake of medium-sized silver nanoparticles into tHp-I cells. Microchemical Journal, I 20, 45-50.

KRYSTEK, P. \& RITSEMA, R. 2002. Determination of uranium in urine - measurement of isotope ratios and quantification by use of inductively coupled plasma mass spectrometry. Anal Bioanal Chem, 374, 226-9.

KRYSTEK, P. \& RITSEMA, R. 2009a. The Determination of Selected Elements in Groundwater by High Resolution Inductively Coupled Plasma Mass Spectrometry - A Validation Assessment. Int J Environ Anal Chem, 89, 33 I-345.

KRYSTEK, P. \& RITSEMA, R. 2009b. An incident study about acute and chronic human exposure to uranium by high-resolution inductively coupled plasma mass spectrometry (hr-iCp-ms). Int J Hyg Environ Health, 2 I2, 76-8I.

KRYSTEK, P., ULRICH, A., GARCIA, C. C., MANOHAR, S. \& RITSEMA, R. 2o I r. Application of Plasma Spectrometry for the Analysis of Engineered Nanoparticles in Suspensions and Products. $J$ Atom Anal Spectrom, 26, I70 I-I 72 I.

LABORDA, F., BOLEA, E. \& JIMENEZ-LAMANA, J. 20I4. Single particle inductively coupled plasma mass spectrometry: a powerful tool for nanoanalysis. Anal Chem, 86, 2270-8.

LABORDA, F., JIMENEZ-LAMANA, J., BOLEA, E. \& CASTILLO, J. R. 2oi i. Selective Identification, Characterization and Determination of Dissolved Silver(I) and Silver Nanoparticles Based on Single Particle Detection by Inductively Coupled Plasma Mass Spectrometry. J Anal At Spectrom, 26, I362-I37I.

LAI, S. K., O’HANLON, D. E., HARROLD, S., MAN, S. T., WANG, Y. Y., CONE, R. \& HANES, J. 2007. Rapid transport of large polymeric nanoparticles in fresh undiluted human mucus. Proc Natl Acad Sci US A, 104, I482-7.

LANDSIEDEL, R., KAPP, M. D., SCHULZ, M., WIENCH, K. \& OESCH, F. 2009. Genotoxicity investigations on nanomaterials: methods, preparation and characterization of test material, potential artifacts and limitations--many questions, some answers. Mutat Res, 68I, 24I-58.

LANDSIEDEL, R., MA-HOCK, L., HOFMANN, T., WIEMANN, M., STRAUSS, V., TREUMANN, S., WOHLLEBEN, W., GROTERS, S., WIENCH, K. \& VAN RAVENZWAAY, B. 20I 4a. Application of short-term inhalation studies to assess the inhalation toxicity of nanomaterials. Part Fibre Toxicol, I I, I6.

LANDSIEDEL, R., MA-HOCK, L., KROLL, A., HAHN, D., SCHNEKENBURGER, J., WIENCH, K. \& WOHLLEBEN, W. 2ого. Testing metal-oxide nanomaterials for human safety. Adv Mater, 22, 260 I-27.

LANDSIEDEL, R., SAUER, U. G., MA-HOCK, L., SCHNEKENBURGER, J. \& WIEMANN, M. 20 I 4 b. Pulmonary toxicity of nanomaterials: a critical comparison of published in vitro assays and in vivo inhalation or instillation studies. Nanomedicine (Lond), 9, 2557-85.

LANKVELD, D. P., OOMEN, A. G., KRYSTEK, P., NEIGH, A., TROOST - DE JONG, A., NOORLANDER, C., HAGENS, W. I. \& DE JONG, W. H. 2o Io. Effect of Size on Tissue Distribution and Accumulation of Silver Nanoparticles. Biomaterials, 3 I, 8350-836I.

LANKVELD, D. P., RAYAVARAPU, R. G., KRYSTEK, P., OOMEN, A. G., VERHAREN, H. W., VAN LEEUWEN, T. G., DE JONG, W. H. \& MANOHAR, S. 2oi I. Blood clearance and tissue distribution of PEGylated and non-PEGylated gold nanorods after intravenous administration in rats. Nanomedicine (Lond), 6, 339-49.

LEE, H., WILLIAMS, S. K., ALLISON, S. D. \& ANCHORDOQUY, T. J. 200 I. Analysis of selfassembled cationic lipid-DNA gene carrier complexes using flow field-flow fractionation and light scattering. Anal Chem, 73, 837-43.

LEE, H., WILLIAMS, S. K., WAHL, K. L. \& VALENTINE, N. B. 2003. Analysis of whole bacterial cells by flow field-flow fractionation and matrix-assisted laser desorption/ionization time-of-flight mass spectrometry. Anal Chem, 75, 2746-52.

LEE, W.-C., HWANG, J.-J., TSENG, Y.-L., WANG, H.-E., CHANG, Y.-F., LU, Y.-C., TING, G., WHANG-PENG, J. \& WANG, S.-J. 2006. Therapeutic efficacy evaluation of I I in-VNB-liposome 
on human colorectal adenocarcinoma HT-29/luc mouse xenografts. Nuclear Instruments and Methods in Physics Research Section A: Accelerators, Spectrometers, Detectors and Associated Equipment, 569, 497-504. LEHMANN, A. D., DAUM, N., BUR, M., LEHR, C. M., GEHR, P. \& ROTHEN-RUTISHAUSER, B. M. 20I I. An in vitro triple cell co-culture model with primary cells mimicking the human alveolar epithelial barrier. Eur J Pharm Biopharm, 77, 398-406.

LENZ, A. G., KARG, E., BRENDEL, E., HINZE-HEYN, H., MAIER, K. L., EICKELBERG, O., STOEGER, T. \& SCHMID, O. 2013. Inflammatory and oxidative stress responses of an alveolar epithelial cell line to airborne zinc oxide nanoparticles at the air-liquid interface: a comparison with conventional, submerged cell-culture conditions. Biomed Res Int, $2013,652632$.

LENZ, A. G., KARG, E., LENTNER, B., DITTRICH, V., BRANDENBERGER, C., ROTHENRUTISHAUSER, B., SCHULZ, H., FERRON, G. A. \& SCHMID, O. 2009. A dose-controlled system for air-liquid interface cell exposure and application to zinc oxide nanoparticles. Part Fibre Toxicol, 6, 32 .

LEO, B. F., CHEN, S., KYO, Y., HERPOLDT, K. L., TERRILL, N. J., DUNLOP, I. E., MCPHAIL, D. S., SHAFFER, M. S., SCHWANDER, S., GOW, A., ZHANG, J., CHUNG, K. F., TETLEY, T. D., PORTER, A. E. \& RYAN, M. P. 20I3. The stability of silver nanoparticles in a model of pulmonary surfactant. Environ Sci Technol, 47, I I 232-40.

LESNIAK, A., FENAROLI, F., MONOPOLI, M. P., ABERG, C., DAWSON, K. A. \& SALVATI, A. 20I2. Effects of the presence or absence of a protein corona on silica nanoparticle uptake and impact on cells. ACS Nano, 6, 5845-57.

LI, N., XIA, T. \& NEL, A. E. 2008. The role of oxidative stress in ambient particulate matter-induced lung diseases and its implications in the toxicity of engineered nanoparticles. Free Radic Biol Med, 44, I689-99.

LIMBACH, L. K., WICK, P., MANSER, P., GRASS, R. N., BRUININK, A. \& STARK, W. J. 2007. Exposure of engineered nanoparticles to human lung epithelial cells: influence of chemical composition and catalytic activity on oxidative stress. Environ Sci Technol, 4I, 4I 58-63.

LINGLAN, M., JINFANG, Z., JUE, W., YAMMEI, D., HUITING, L., NA, L., JINGYING, Y., JIE, R., WANG, H. \& FASHUI, H. 2009. The Acute Liver Inhury in Mice Caused by Nano-anatase TiO2. Nanoscale Res Lett, 4, I 275 - I 285 .

LIU, J., ANDYA, J. D. \& SHIRE, S. J. 2006. A critical review of analytical ultracentrifugation and field flow fractionation methods for measuring protein aggregation. Aaps j, 8, E580-9.

LIU, W., WU, Y., WANG, C., LI, H. C., WANG, T., LIAO, C. Y., CUI, L., ZHOU, Q. F., YAN, B. \& JIANG, G. B. 20 Iо. Impact of silver nanoparticles on human cells: effect of particle size. Nanotoxicology, 4, 319-30.

LOESCHNER, K., BRABRAND, M. S., SLOTH, J. J. \& LARSEN, E. H. 2or 4. Use of alkaline or enzymatic sample pretreatment prior to characterization of gold nanoparticles in animal tissue by single-particle ICPMS. Anal Bioanal Chem, 406, 3845-5 I.

LOK, C. N., HO, C. M., CHEN, R., HE, Q. Y., YU, W. Y., SUN, H., TAM, P. K., CHIU, J. F. \& CHE, C. M. 2007. Silver nanoparticles: partial oxidation and antibacterial activities. J Biol Inorg Chem, I2, 527-34.

LOMER, M. C., THOMPSON, R. P., COMMISSO, J., KEEN, C. L. \& POWELL, J. J. 2000. Determination of titanium dioxide in foods using inductively coupled plasma optical emission spectrometry. Analyst, I 25, 2339-43.

LOZANO, O., MEJIA, J., MASEREEL, B., TOUSSAINT, O., LISON, D. \& LUCAS, S. 2 o 2. Development of a pixE analysis method for the determination of the biopersistence of $\mathrm{SiC}$ and $\mathrm{TiC}$ nanoparticles in rat lungs. Nanotoxicology, 6, 263-7I.

LOZANO, O., OLIVIER, T., DOGNE, J. M. \& LUCAS, S. 2013. The use of pixE for engineered nanomaterials quantification in complex matrices. Journal of Physics, Conference series 429.

LUBICK, N. 2008. Nanosilver toxicity: ions, nanoparticles--or both? Environ Sci Technol, 42, 861 7. LUNDQVIST, M., STIGLER, J., ELIA, G., LYNCH, I., CEDERVALL, T. \& DAWSON, K. A. 2008. Nanoparticle size and surface properties determine the protein corona with possible implications for biological impacts. Proc Natl Acad Sci US A, I05, I 4265-70. 
LUYTS, K., NAPIERSKA, D., DINSDALE, D., KLEIN, S. G., SERCHI, T. \& HOET, P. H. 20 I 5. A coculture model of the lung-blood barrier: the role of activated phagocytic cells. Toxicol In Vitro, 29, 234-4I.

MA-HOCK, L., BRILL, S., WOHLLEBEN, W., FARIAS, P. M., CHAVES, C. R., TENORIO, D. P., FONTES, A., SANTOS, B. S., LANDSIEDEL, R., STRAUSS, V., TREUMANN, S. \& RAVENZWAAY, B. 20I2. Short term inhalation toxicity of a liquid aerosol of $\mathrm{CdS} / \mathrm{Cd}(\mathrm{OH})$ core shell quantum dots in male Wistar rats. Toxicol Lett, 208, I I 5-24.

MA-HOCK, L., STRAUSS, V., TREUMANN, S., KUTTLER, K., WOHLLEBEN, W., HOFMANN, T., GROTERS, S., WIENCH, K., VAN RAVENZWAAY, B. \& LANDSIEDEL, R. 20I3. Comparative inhalation toxicity of multi-wall carbon nanotubes, graphene, graphite nanoplatelets and low surface carbon black. Part Fibre Toxicol, , o, 23.

MA-HOCK, L., TREUMANN, S., STRAUSS, V., BRILL, S., LUIZI, F., MERTLER, M., WIENCH, K., GAMER, A. O., VAN RAVENZWAAY, B. \& LANDSIEDEL, R. 2009. Inhalation toxicity of multiwall carbon nanotubes in rats exposed for 3 months. Toxicol Sci, I I 2, 468-8 I.

MA, R., LEVARD, C., MARINAKOS, S. M., CHENG, Y., LIU, J., MICHEL, F. M., BROWN, G. E. \& LOWRY, G. V. 20I 2. Size-controlled dissolution of organic-coated silver nanoparticles. Environ Sci Technol, 46, 752-9.

MADLOVA, M., JONES, S. A., ZWERSCHKE, I., MA, Y., HIDER, R. C. \& FORBES, B. 2009. Poly(vinyl alcohol) nanoparticle stability in biological media and uptake in respiratory epithelial cell layers in vitro. Eur J Pharm Biopharm, 72, 437-43.

MAHTO, S. K., CHARWAT, V., ERTL, P., ROTHEN-RUTISHAUSER, B., RHEE, S. W. \& SZNITMAN, J. 2015. Microfluidic platforms for advanced risk assessments of nanomaterials. Nanotoxicology, 9, 38I-95.

MARX, U., WALLES, H., HOFFMANN, S., LINDNER, G., HORLAND, R., SONNTAG, F., KLOTZBACH, U., SAKHAROV, D., TONEVITSKY, A. \& LAUSTER, R. 2o I 2. 'Humanon-a-chip' developments: a translational cutting-edge alternative to systemic safety assessment and efficiency evaluation of substances in laboratory animals and man? Altern Lab Anim, 40, 235-57.

MAYNARD, A. D. \& KUEMPEL, E. D. 2005. Airborne nanostructured particles and occupational health. J Nanoparticle Res, 7, 587-6I 4.

MAYNARD, R. L., DONALDSON, K. \& TETLEY, T. D. 2013. Type i pulmonary epithelial cells: a new compartment involved in the slow phase of particle clearance from alveoli. Nanotoxicology, 7 , 350-I.

MCCLELLAN, R. O. \& HENDERSON, R. F. I995. Concepts in Inhalation Toxicology, Washington, Taylor \& Francis.

MERCER, R. R., HUBBS, A. F., SCABILLONI, J. F., WANG, L., BATTELLI, L. A., SCHWEGLERBERRY, D., CASTRANOVA, V. \& PORTER, D. W. 20I0. Distribution and persistence of pleural penetrations by multi-walled carbon nanotubes. Part Fibre Toxicol, 7, 28.

MITRANO, D. M., LESHER, E. K., BEDNAR, A., MONSERUD, J., HIGGINS, C. P. \& RANVILLE, J. F. 20I2. Detecting nanoparticulate silver using single-particle inductively coupled plasma-mass spectrometry. Environ Toxicol Chem, 3 I, I I 5-2 I.

MOHAMMAD, A. K., AMAYREH, L. K., MAZZARA, J. M. \& REINEKE, J. J. 20I 3. Rapid lymph accumulation of polystyrene nanoparticles following pulmonary administration. Pharm Res,

30, 424-34.

MOLLER, P., JACOBSEN, N. R., FOLKMANN, J. K., DANIELSEN, P. H., MIKKELSEN, L., HEMMINGSEN, J. G., VESTERDAL, L. K., FORCHHAMMER, L., WALLIN, H. \& LOFT, S. 20 Io. Role of oxidative damage in toxicity of particulates. Free Radic Res, 44, I-46.

MÖLLER, W., KREYLING, W. G., SCHMID, O., SEMMLER-BEHNKE, M. \& SCHULZ, H. 20 Iо. Deposition, retention and clearance, and translocation of inhaled fine and nano-sized particles in the respiratory tract. In: GEHR, P., MÜHLFELD, C., ROTHEN-RUTISHAUSER, B. \& BLANK, F. (eds.) Particle-Lung Interactions, second ed. USA: Informa Healthcare USA, Inc.

MONTASER, A. 1998. Inductively Coupled Plasma Mass Spectrometry, New York, Wiley-VCH. 
MONTEILlER, C., TRAN, L., MACNEE, W., FAUX, S., JONES, A., MILlER, B. \& DONALDSON, K. 2007. The pro-inflammatory effects of low-toxicity low-solubility particles, nanoparticles and fine particles, on epithelial cells in vitro: the role of surface area. Occup Environ Med, 64, 609-I 5 .

MORAES, C., MEHTA, G., LESHER-PEREZ, S. C. \& TAKAYAMA, S. 2o I 2. Organs-on-a-chip: a focus on compartmentalized microdevices. Ann Biomed Eng, 4O, I 2 I I-27.

MORGAN, A., TALBOT, R. J. \& HOLMES, A. I978. Significance of fibre length in the clearance of asbestos fibres from the lung. Br J Ind Med, 35, I 46-53.

MORONES, J. R., ELECHIGUERRA, J. L., CAMACHO, A., HOLT, K., KOURI, J. B., RAMIREZ, J. T. \& YACAMAN, M. J. 2005. The bactericidal effect of silver nanoparticles. Nanotechnology, I6, 2346-53.

MORROW, P. E. 1988. Possible mechanisms to explain dust overloading of the lungs. Fundam Appl Toxicol, io, 369-84.

MORROW, P. E. I 992. Dust overloading of the lungs: update and appraisal. Toxicol Appl Pharmacol, I I3, I-I 2 .

MUHLFELD, C., GEHR, P. \& ROTHEN-RUTISHAUSER, B. 2008a. Translocation and cellular entering mechanisms of nanoparticles in the respiratory tract. Swiss Med Wkly, I38, 387-9I.

MUHLFELD, C., ROTHEN-RUTISHAUSER, B., BLANK, F., VANHECKE, D., OCHS, M. \& GEHR, P. 2008b. Interactions of nanoparticles with pulmonary structures and cellular responses. $\mathrm{Am}$ J Physiol Lung Cell Mol Physiol, 294, L8 I7-29.

MUKHERJEE, D., LEO, B. F., ROYCE, S. G., PORTER, A. E., RYAN, M. P., SCHWANDER, S., CHUNG, K. F., TETLEY, T. D., ZHANG, J. \& GEORGOPOULOS, P. G. 20I4. Modeling physicochemical interactions affecting in vitro cellular dosimetry of engineered nanomaterials: application to nanosilver. J Nanopart Res, I6, 26 I6.

MÜlHOPT, S., DIABATE, S., KREBS, T., WEISS, C. \& PAUR, H. R. 2009. Lung toxicity determination by in vitro exposure at the air iquid interface with an integrated online dose measurement. In: PHYSICS, J. O. (ed.) Nanosafe 2008: International Conference on Safe production and use of nanomaterials. IOP Publishing.

MULLER, L., RIEDIKER, M., WICK, P., MOHR, M., GEHR, P. \& ROTHEN-RUTISHAUSER, B. 20IO. Oxidative stress and inflammation response after nanoparticle exposure: differences between human lung cell monocultures and an advanced three-dimensional model of the human epithelial airways. J R Soc Interface, 7 Suppl I, S27-40.

MURDOCK, R. C., BRAYDICH-STOLLE, L., SCHRAND, A. M., SCHLAGER, J. J. \& HUSSAIN, S. M. 2008. Characterization of nanomaterial dispersion in solution prior to in vitro exposure using dynamic light scattering technique. Toxicol Sci, I I, 239-53.

MURPHY, F. A., POLAND, C. A., DUfFIN, R., AL-JAMAL, K. T., Ali-BOUCETTA, H., NUNES, A., BYRNE, F., PRINA-MELLO, A., VOLKOV, Y., LI, S., MATHER, S. J., BIANCO, A., PRATO, M., MACNEE, W., WALLACE, W. A., KOSTARELOS, K. \& DONALDSON, K. $20 \mathrm{I}$ I. Length-dependent retention of carbon nanotubes in the pleural space of mice initiates sustained inflammation and progressive fibrosis on the parietal pleura. Am J Pathol, I78, 2587-60o.

NAGY, A., STEINBRUCK, A., GAO, J., DOGGETT, N., HOLLINGSWORTH, J. A. \& IYER, R. 20I2. Comprehensive analysis of the effects of CdSe quantum dot size, surface charge, and functionalization on primary human lung cells. ACS Nano, 6, 4748-62.

NAIM, B., ZBAIDA, D., DAGAN, S., KAPON, R. \& REICH, Z. 2009. Cargo surface hydrophobicity is sufficient to overcome the nuclear pore complex selectivity barrier. $E M B O \mathrm{~J}, 28,2697-705$.

NALAYANDA, D. D., WANG, Q., FULTON, W. B., WANG, T. H. \& ABDULLAH, F. 20 го. Engineering an artificial alveolar-capillary membrane: a novel continuously perfused model within microchannels. J Pediatr Surg, 45, 45-5 I.

NANOTECHNOLOGIES, P. O. E. 20I4. Consumer Products Inventory [Online]. Available: http:// www.nanotechproject.org/cpi [Accessed June 20I4].

NAPIERSKA, D., THOMASSEN, L. C., VANAUDENAERDE, B., LUYTS, K., LISON, D., 
MARTENS, J. A., NEMERY, B. \& HOET, P. H. 20 I 2. Cytokine production by co-cultures exposed to monodisperse amorphous silica nanoparticles: the role of size and surface area. Toxicol Lett, 2 I I, 98-I04.

NEL, A., XIA, T., MADLER, L. \& LI, N. 2006. Toxic potential of materials at the nanolevel. Science, 3 I I, 622-7.

NEL, A. E., MADLER, L., VELEGOL, D., XIA, T., HOEK, E. M., SOMASUNDARAN, P., KLAESSIG, F., CASTRANOVA, V. \& THOMPSON, M. 2009. Understanding biophysicochemical interactions at the nano-bio interface. Nat Mater, 8, 543-57.

NICKEL, C., ANGELSTORF, J., BIENERT, R., BURKART, C., GABSCH, S., GIEBNER, S. \& HAASE, A. 20 I 4. Dynamic light-scattering measurement comparability of nanomaterial suspensions. J Nanopart Res, I6.

NICOD, L. P. 1999. Pulmonary defence mechanisms. Respiration, 66, 2-I I.

NOEL, A., MAGHNI, K., ClOUTIER, Y., DION, C., WILKINSON, K. J., HALle, S., TARDIF, R. \& TRUCHON, G. 20 I 2. Effects of inhaled nano-TiO2 aerosols showing two distinct agglomeration states on rat lungs. Toxicol Lett, 2 I4, I09-I 9.

NOLAN, R. P., LANGER, A. M., HARINGTON, J. S., OSTER, G. \& SELIKOFF, I. J. I98 I. Quartz hemolysis as related to its surface functionalities. Environ Res, 26, 503-20.

NORRIS, D. A., PURI, N. \& SINKO, P. J. I998. The effect of physical barriers and properties on the oral absorption of particulates. Adv Drug Deliv Rev, 34, I35-I 54.

NYMARK, P., CATALAN, J., SUHONEN, S., JARVENTAUS, H., BIRKEDAL, R., CLAUSEN, P. A., JENSEN, K. A., VIPPOLA, M., SAVOLAINEN, K. \& NORPPA, H. 2013. Genotoxicity of polyvinylpyrrolidone-coated silver nanoparticles in BEAS 2 B cells. Toxicology, 3 I 3, 38-48.

OBERDORSTER, G. I989. Dosimetric principles for extrapolating results of rat inhalation studies to humans, using an inhaled Ni compound as an example. Health Phys, 57 Suppl I, 21 3-20.

OBERDORSTER, G., FERIN, J. \& LEHNERT, B. E. I 994. Correlation between particle size, in vivo particle persistence, and lung injury. Environ Health Perspect, Io2 Suppl 5, I73-9.

OBERDORSTER, G., FINKELSTEIN, J. N., JOHNSTON, C., GELEIN, R., COX, C., BAGGS, R. \& ELDER, A. C. 2000. Acute pulmonary effects of ultrafine particles in rats and mice. Res Rep Health Eff Inst, 5-74; disc 75-86.

OBERDORSTER, G., MAYNARD, A., DONALDSON, K., CASTRANOVA, V., FITZPATRICK, J., AUSMAN, K., CARTER, J., KARN, B., KREYLING, W., LAI, D., OLIN, S., MONTEIRORIVIERE, N., WARHEIT, D. \& YANG, H. 2005a. Principles for characterizing the potential human health effects from exposure to nanomaterials: elements of a screening strategy. Part Fibre Toxicol, 2,8 .

OBERDORSTER, G., OBERDORSTER, E. \& OBERDORSTER, J. 2005b. Nanotoxicology: an emerging discipline evolving from studies of ultrafine particles. Environ Health Perspect, I I 3, 823-39. OBERDORSTER, G. \& POTT, F. 1987. Extrapolation from rat studies with environmental tobacco smoke (ETS) to humans: comparison of particle mass deposition and of clearance behavior of ETS compounds. Toxicol Lett, 35, I07-I 2.

OBERDORSTER, G., SHARP, Z., ATUDOREI, V., ELDER, A., GELEIN, R., KREYLING, W. \& COX, C. 2004. Translocation of inhaled ultrafine particles to the brain. Inhal Toxicol, I6, 437-45. OOMEN, A. G., M, B., ENGELEN, J. \& SIPS, A. J. 20 I I. Nanomaterial in consumer products : Detection, characterisation and interpretation. RIVM Rapport $32002900 \mathrm{I}$.

OSTROWSKI, A., NORDMEYER, D., BOREHAM, A., HOLZHAUSEN, C., MUNDHENK, L., GRAF, C., MEINKE, M. C., VOGT, A., HADAM, S., LADEMANN, J., RUHL, E., ALEXIEV, U. \& GRUBER, A. D. 2015. Overview about the localization of nanoparticles in tissue and cellular context by different imaging techniques. Beilstein J Nanotechnol, 6, 263-80.

PACE, H. E., ROGERS, N. J., JAROLIMEK, C., COLEMAN, V. A., GRAY, E. P., HIGGINS, C. P. \& RANVILLE, J. F. 20 I2. Single particle inductively coupled plasma-mass spectrometry: a performance evaluation and method comparison in the determination of nanoparticle size. Environ Sci Technol, 46, I 2272-80. 
PACE, H. E., ROGERS, N. J., JAROLimeK, C., COlEMAN, V. A., HigGinS, C. P. \& RANVILLE, J. F. 20II. Determining transport efficiency for the purpose of counting and sizing nanoparticles via single particle inductively coupled plasma mass spectrometry. Anal Chem, 83, 936I-9. PANAS, A., COMOUTH, A., SAATHOFF, H., LEISNER, T., AL-RAWI, M., SIMON, M., SEEMANN, G., DOSSEL, O., MULHOPT, S., PAUR, H. R., FRITSCH-DECKER, S., WEISS, C. \& DIABATE, S. 20I4. Silica nanoparticles are less toxic to human lung cells when deposited at the air-liquid interface compared to conventional submerged exposure. Beilstein J Nanotechnol, 5, I $590-602$.

PAPRITZ, M., POHL, C., WUBBEKE, C., MOISCH, M., HOFMANN, H., HERMANNS, M. I., THIERMANN, H., KIRKPATRICK, C. J. \& KEHE, K. 2о Io. Side-specific effects by cadmium exposure: apical and basolateral treatment in a coculture model of the blood-air barrier. Toxicol Appl Pharmacol, 245, 36I-9.

PARANJPE, M. \& MULLER-GOYMANN, C. C. 2014 . Nanoparticle-mediated pulmonary drug delivery: a review. Int J Mol Sci, I 5, $5852-73$.

PARK, E. J., YI, J., CHUNG, K. H., RYU, D. Y., CHOI, J. \& PARK, K. 2008. Oxidative stress and apoptosis induced by titanium dioxide nanoparticles in cultured BEAS-2B cells. Toxicol Lett, I80, 222-9. PARK, E. J., YI, J., KIM, Y., CHOI, K. \& PARK, K. 20Iо. Silver nanoparticles induce cytotoxicity by a Trojan-horse type mechanism. Toxicol In Vitro, 24, 872-8.

PARK, M. V., ANNEMA, W., SALVATI, A., LESNIAK, A., ELSAESSER, A., BARNES, C., MCKERR, G., HOWARD, C. V., LYNCH, I., DAWSON, K. A., PIERSMA, A. H. \& DE JONG, W. H. 2009. In vitro developmental toxicity test detects inhibition of stem cell differentiation by silica nanoparticles. Toxicol Appl Pharmacol, 240, го8-i6.

PARK, M. V., NEIGH, A. M., VERMEULEN, J. P., DE IA FONTEYNE, L. J., VERHAREN, H. W., BRIEDE, J. J., VAN LOVEREN, H. \& DE JONG, W. H. 20 I I. The effect of particle size on the cytotoxicity, inflammation, developmental toxicity and genotoxicity of silver nanoparticles. Biomaterials, 32, 98 I $0-7$.

PAULUHN, J. 2009a. Pulmonary toxicity and fate of agglomerated 10 and $40 \mathrm{~nm}$ aluminum oxyhydroxides following 4-week inhalation exposure of rats: toxic effects are determined by agglomerated, not primary particle size. Toxicol Sci, I09, I 52-67.

PAULUHN, J. 2009b. Retrospective analysis of 4-week inhalation studies in rats with focus on fate and pulmonary toxicity of two nanosized aluminum oxyhydroxides (boehmite) and pigment-grade iron oxide (magnetite): the key metric of dose is particle mass and not particle surface area. Toxicology, 259, I40-8.

PAULUHN, J. 20I0. Subchronic I3-week inhalation exposure of rats to multiwalled carbon nanotubes: toxic effects are determined by density of agglomerate structures, not fibrillar structures. Toxicol Sci, I I $3,226-42$.

PAULUHN, J. 20 I I. Poorly soluble particulates: searching for a unifying denominator of nanoparticles and fine particles for DNEL estimation. Toxicology, 279, I76-88.

PENICHE, C., ZALDIVAR, D., PAZOS, M., PAZ, S., BULAY, A. \& SAN ROMAN, J. I 993. Study of the Thermal Degradation of Poly(N-vinyl-2-pyrrolidone) by Thermogravimetry-FTIR. Journal of Applied Polymer Science, 50, 9.

PERETYAZHKO, T. S., ZHANG, Q. \& COLVIN, V. L. 20 I 4. Size-controlled dissolution of silver nanoparticles at neutral and acidic $\mathrm{pH}$ conditions: kinetics and size changes. Environ Sci Technol, 48, I I 954-6I.

PETERS, R. J., RIVERA, Z. H., VAN BEMMEL, G., MARVIN, H. J., WEIGEL, S. \& BOUWMEESTER, H. 20I4a. Development and validation of single particle iCp-ms for sizing and quantitative determination of nano-silver in chicken meat. Anal Bioanal Chem, 406, 3875-85.

PETERS, R. J., VAN BEMMEL, G., HERRERA-RIVERA, Z., HELSPER, H. P., MARVIN, H. J., WEIGEL, S., TROMP, P. C., OOMEN, A. G., RIETVELD, A. G. \& BOUWMEESTER, H. 2014 b. Characterization of titanium dioxide nanoparticles in food products: analytical methods to define nanoparticles. J Agric Food Chem, 62, 6285-93. 
PHALEN, R. F., MENDEZ, L. B. \& OLDHAM, M. J. 201о. New developments in aerosol dosimetry. Inhal Toxicol, 22 Suppl 2, 6-I 4.

PILCER, G. \& AMIGHI, K. 20Iо. Formulation strategy and use of excipients in pulmonary drug delivery. Int J Pharm, 392, I-I 9.

POLAND, C. A., DUFFIN, R., KINLOCH, I., MAYNARD, A., WALLACE, W. A., SEATON, A., STONE, V., BROWN, S., MACNEE, W. \& DONALDSON, K. 2008. Carbon nanotubes introduced into the abdominal cavity of mice show asbestos-like pathogenicity in a pilot study. Nat Nanotechnol, 3, 423-8.

PORTER, D. W., HUBBS, A. F., CHEN, B. T., MCKINNEY, W., MERCER, R. R., WOLFARTH, M. G., BATTELLI, L., WU, N., SRIRAM, K., LEONARD, S., ANDREW, M., WILLARD, P., TSURUOKA, S., ENDO, M., TSUKADA, T., MUNEKANE, F., FRAZER, D. G. \& CASTRANOVA, V. 20I2a. Acute pulmonary dose-responses to inhaled multi-walled carbon nanotubes. Nanotoxicology.

PORTER, D. W., HUBBS, A. F., MERCER, R. R., WU, N., WOLFARTH, M. G., SRIRAM, K., LEONARD, S., BATTELLI, L., SCHWEGLER-BERRY, D., FRIEND, S., ANDREW, M., CHEN, B. T., TSURUOKA, S., ENDO, M. \& CASTRANOVA, V. 20io. Mouse pulmonary doseand time course-responses induced by exposure to multi-walled carbon nanotubes. Toxicology, 269, I36-47.

PORTER, D. W., WU, N., HUBBS, A., MERCER, R., FUNK, K., MENG, F., LI, J., WOLFARTH, M., BATTELLI, L., FRIEND, S., ANDREW, M., HAMILTON, R., SRIRAM, K., YANG, F., CASTRANOVA, V. \& HOLIAN, A. 2or zb. Differential Mouse Pulmonary Dose- and Time Course-Responses to Titanium Dioxide Nanospheres and Nanobelts. Toxicol Sci.

POWERS, K. W., BROWN, S. C., KRISHNA, V. B., WASDO, S. C., MOUDGIL, B. M. \& ROBERTS, S. M. 2006. Research strategies for safety evaluation of nanomaterials. Part VI. Characterization of nanoscale particles for toxicological evaluation. Toxicol Sci, 90, 296-303.

PRATSINIS, A., HERVELLA, P., LEROUX, J. C., PRATSINIS, S. E. \& SOTIRIOU, G. A. 20 I 3. Toxicity of silver nanoparticles in macrophages. Small, 9, 2576-84.

QUADROS, M. E. \& MARR, L. C. 20II. Silver nanoparticles and total aerosols emitted by nanotechnology-related consumer spray products. Environ Sci Technol, 45, I07 I 3-9.

RAEMY, D. O., LIMBACH, L. K., ROTHEN-RUTISHAUSER, B., GRASS, R. N., GEHR, P., BIRBAUM, K., BRANDENBERGER, C., GUNTHER, D. \& STARK, W. J. 2o I I. Cerium oxide nanoparticle uptake kinetics from the gas-phase into lung cells in vitro is transport limited. Eur J Pharm Biopharm, 77, 368-75.

RAJH, T., NEDELJKOVIC, M., CHEN, L. X., POLUEKTOV, O. \& THURNAUER, M. C. I 999. Improving optical and charge separation properties of nanocrystalline $\mathrm{TiO}_{2}$ by surface modification with vitamin C. .J Phys Chem, I03, 35 I 5 .

RATANATHANAWONG, S. K., WILLIAMS, S. \& LEE, D. 2006. Field-flow Fractionation of proteins, Polysaccharides, Synthetic Polymers, and Supramolecular Assemblies. J Sep Sci, 29, I720I732.

REUZEL, P. G., BRUIJNTJES, J. P., FERON, V. J. \& WOUTERSEN, R. A. i99i. Subchronic inhalation toxicity of amorphous silicas and quartz dust in rats. Food Chem Toxicol, 29, 34I-54.

RIEHEMANN, K., SCHNEIDER, S. W., LUGER, T. A., GODIN, B., FERRARI, M. \& FUCHS, H. 2009. Nanomedicine-Challenge and Perspectives. Angewandte Chemie International Edition, 48, 872-897.

RIVM 20I4. PROAST: Software for dose-response modeling and benchmark dose analysis. PROAST 38.9 ed. Bilthoven, the Netherlands.

ROBERTS, J. R., CHAPMAN, R. S., TIRUMALA, V. R., KARIM, A., CHEN, B. T., SCHWEGLER-BERRY, D., STEFANIAK, A. B., LEONARD, S. S. \& ANTONINI, J. M. 20 I I. Toxicological evaluation of lung responses after intratracheal exposure to non-dispersed titanium dioxide nanorods. J Toxicol Environ Health A, 74, 790-8 I0.

ROTHEN-RUTISHAUSER, B., GRASS, R. N., BLANK, F., LIMBACH, L. K., MUHLFELD, C., 
BRANDENBERGER, C., RAEMY, D. O., GEHR, P. \& STARK, W. J. 2009. Direct combination of nanoparticle fabrication and exposure to lung cell cultures in a closed setup as a method to simulate accidental nanoparticle exposure of humans. Environ Sci Technol, 43, 2634-40.

ROTHEN-RUTISHAUSER, B., MUELLER, L., BLANK, F., BRANDENBERGER, C., MUEHLFELD, C. \& GEHR, P. 2008. A newly developed in vitro model of the human epithelial airway barrier to study the toxic potential of nanoparticles. ALTEX, 25, I9I-6.

ROTHEN-RUTISHAUSER, B. M., KIAMA, S. G. \& GEHR, P. 2005. A three-dimensional cellular model of the human respiratory tract to study the interaction with particles. Am J Respir Cell Mol Biol, $32,28 I-9$.

ROURSGAARD, M., POUlSEN, S. S., POUlSEN, L. K., HAMMER, M., JENSEN, K. A., UTSUNOMIYA, S., EWING, R. C., BALIC-ZUNIC, T., NIELSEN, G. D. \& LARSEN, S. T. 20I0. Time-response relationship of nano and micro particle induced lung inflammation. Quartz as reference compound. Hum Exp Toxicol, 29, 91 5-33.

SADAUSKAS, E., JACOBSEN, N. R., DANSCHER, G., STOLTENBERG, M., VOGEL, U., LARSEN, A., KREYLING, W. \& WALLIN, H. 2009. Biodistribution of gold nanoparticles in mouse lung following intratracheal instillation. Chem Cent J, 3, I6.

SADAUSKAS, E., WALLIN, H., STOLTENBERG, M., VOGEL, U., DOERING, P., LARSEN, A. \& DANSCHER, G. 2007. Kupffer cells are central in the removal of nanoparticles from the organism. Part Fibre Toxicol, 4, Io.

SANCHEZ, V. C., JACHAK, A., HURT, R. H. \& KANE, A. B. 2or 2. Biological interactions of graphene-family nanomaterials: an interdisciplinary review. Chem Res Toxicol, 25, I 5-34.

SARLO, K., BLACKBURN, K. L., CLARK, E. D., GROTHAUS, J., CHANEY, J., NEU, S., FLOOD, J., ABBOTT, D., BOHNE, C., CASEY, K., FRYER, C. \& KUHN, M. 2009. Tissue distribution of $20 \mathrm{~nm}$, I00 $\mathrm{nm}$ and $1000 \mathrm{~nm}$ fluorescent polystyrene latex nanospheres following acute systemic or acute and repeat airway exposure in the rat. Toxicology, 263, I 17-26.

SARMIENTO-GONZALEZ, A., ENCINAR, J. R., MARCHANTE-GAYON, J. M. \& SANZMEDEL, A. 2009. Titanium levels in the organs and blood of rats with a titanium implant, in the absence of wear, as determined by double-focusing iCp-ms. Anal Bioanal Chem, 393, 335-43.

SAUNDERS, M. 2009. Transplacental transport of nanomaterials. Wiley Interdiscip Rev Nanomed Nanobiotechnol, I, 67I-84.

SAVI, M., KALBERER, M., LANG, D., RYSER, M., FIERZ, M., GASCHEN, A., RICKA, J. \& GEISER, M. 2008. A novel exposure system for the efficient and controlled deposition of aerosol particles onto cell cultures. Environ Sci Technol, 42, 5667-74.

SAYES, C. M., REED, K. L., GLOVER, K. P., SWAIN, K. A., OSTRAAT, M. L., DONNER, E. M. \& WARHEIT, D. B. 20Io. Changing the dose metric for inhalation toxicity studies: short-term study in rats with engineered aerosolized amorphous silica nanoparticles. Inhal Toxicol, 22, 348-54.

SAYES, C. M., WAHI, R., KURIAN, P. A., LIU, Y., WEST, J. L., AUSMAN, K. D., WARHEIT, D. B. \& COLVIN, V. L. 2006. Correlating nanoscale titania structure with toxicity: a cytotoxicity and inflammatory response study with human dermal fibroblasts and human lung epithelial cells. Toxicol Sci, 92, I74-85.

SCENIHR 20Io. Basis for the Definition of the Term 'nanomaterial'. Brussels, Belgium: European Commission.

SCHIMEK, K., BUSEK, M., BRINCKER, S., GROTH, B., HOFFMANN, S., LAUSTER, R., LINDNER, G., LORENZ, A., MENZEL, U., SONNTAG, F., WALLES, H., MARX, U. \& HORLAND, R. 20I3. Integrating biological vasculature into a multi-organ-chip microsystem. Lab Chip, I3, 3588-98.

SCHINWALD, A., CHERNOVA, T. \& DONALDSON, K. 20 I 2a. Use of silver nanowires to determine thresholds for fibre length-dependent pulmonary inflammation and inhibition of macrophage migration in vitro. Part Fibre Toxicol, 9, 47.

SCHINWALD, A., MURPHY, F. A., JONES, A., MACNEE, W. \& DONALDSON, K. 2or 2 b. Graphene-based nanoplatelets: a new risk to the respiratory system as a consequence of their unusual aerodynamic properties. ACS Nano, 6, 736-46. 
SCHINWALD, A., MURPHY, F. A., PRINA-MELLO, A., POLAND, C. A., BYRNE, F., MOVIA, D., GLASS, J. R., DICKERSON, J. C., SCHULTZ, D. A., JEFFREE, C. E., MACNEE, W. \& DONALDSON, K. 20I2c. The threshold length for fiber-induced acute pleural inflammation: shedding light on the early events in asbestos-induced mesothelioma. Toxicol Sci, I 28, 46I-70.

SEMMLER, M., SEITZ, J., ERBE, F., MAYER, P., HEYDER, J., OBERDORSTER, G. \& KREYLING, W. G. 2004. Long-term clearance kinetics of inhaled ultrafine insoluble iridium particles from the rat lung, including transient translocation into secondary organs. Inhal Toxicol, I6, 453-9. SHANNAHAN, J. H., PODILA, R., ALDOSSARI, A. A., EMERSON, H., POWELL, B. A., KE, P. C., RAO, A. M. \& BROWN, J. M. 2015. Formation of a protein corona on silver nanoparticles mediates cellular toxicity via scavenger receptors. Toxicol Sci, I 43, I 36-46.

SHVEDOVA, A. A., KISIN, E. R., MERCER, R., MURRAY, A. R., JOHNSON, V. J., POTAPOVICH, A. I., TYURINA, Y. Y., GORELIK, O., AREPALLI, S., SCHWEGLER-BERRY, D., HUBBS, A. F., ANTONINI, J., EVANS, D. E., KU, B. K., RAMSEY, D., MAYNARD, A., KAGAN, V. E., CASTRANOVA, V. \& BARON, P. 2005. Unusual inflammatory and fibrogenic pulmonary responses to single-walled carbon nanotubes in mice. Am J Physiol Lung Cell Mol Physiol, 289, L698-708.

SILVEIRA, J. R., RAYMOND, G. J., HUGHSON, A. G., RACE, R. E., SIM, V. L., HAYES, S. F. \& CAUGHEY, B. 2005. The most infectious prion protein particles. Nature, 437, 257-6I.

SIMKO, M. \& MATTSSON, M. O. 20I0. Risks from accidental exposures to engineered nanoparticles and neurological health effects: a critical review. Part Fibre Toxicol, 7, 42.

SIMON-DECKERS, A., GOUGET, B., MAYNE-L'HERMITE, M., HERLIN-BOIME, N., REYNAUD, C. \& CARRIERE, M. 2008. In vitro investigation of oxide nanoparticle and carbon nanotube toxicity and intracellular accumulation in A549 human pneumocytes. Toxicology, 253, I 37-46.

SINGH, S., KUMAR, A., KARAKOTI, A., SEAL, S. \& SELF, W. T. 20 Io. Unveiling the mechanism of uptake and sub-cellular distribution of cerium oxide nanoparticles. Mol Biosyst, 6, I 8 I 3-20.

SINHA, R., LE GAC, S., VERDONSCHOT, N., VAN DEN BERG, A., KOOPMAN, B. \& ROUWKEMA, J. 20I 5 . A medium throughput device to study the effects of combinations of surface strains and fluid-flow shear stresses on cells. Lab Chip, I 5, 429-39.

SLOB, W. 2002. Dose-response modeling of continuous endpoints. Toxicol Sci, 66, 298-3 I 2.

SLOB, W. 20 I 4a. Benchmark dose and the three Rs. Part I. Getting more information from the same number of animals. Crit Rev Toxicol, 44, 557-67.

SLOB, W. 20I4b. Benchmark dose and the three Rs. Part II. Consequences for study design and animal use. Crit Rev Toxicol, 44, 568-80.

SONAVANE, G., TOMODA, K. \& MAKINO, K. 2008. Biodistribution of colloidal gold nanoparticles after intravenous administration: effect of particle size. Colloids Surf B Biointerfaces, 66, 274-80.

SONG, K. S., SUNG, J. H., JI, J. H., LEE, J. H., LEE, J. S., RYU, H. R., LEE, J. K., CHUNG, Y. H., PARK, H. M., SHIN, B. S., CHANG, H. K., KELMAN, B. \& YU, I. J. 2or 2. Recovery from silver-nanoparticle-exposure-induced lung inflammation and lung function changes in Sprague Dawley rats. Nanotoxicology.

SOTIRIOU, G. A. \& PRATSINIS, S. E. 20I0. Antibacterial activity of nanosilver ions and particles. Environ Sci Technol, 44, 5649-54.

SOTIRIOU, G. A. \& PRATSINIS, S. E. 20 I I. Engineering nanosilver as an antibacterial, biosensor and bioimaging material. Curr Opin Chem Eng, I, 3-Io.

STEBOUNOVA, L. V., ADAMCAKOVA-DODD, A., KIM, J. S., PARK, H., O'SHAUGHNESSY, P. T., GRASSIAN, V. H. \& THORNE, P. S. 20 i Ia. Nanosilver induces minimal lung toxicity or inflammation in a subacute murine inhalation model. Part Fibre Toxicol, 8, 5 .

STEBOUNOVA, L. V., GUIO, E. \& GRASSIAN, V. H. 20I Ib. Silver nanoparticles in simulated biological media: a study of aggregation, sedimentation, and dissolution. J Nanopar Res, I3, I 2.

STOEGER, T., REINHARD, C., TAKENAKA, S., SCHROEPPEL, A., KARG, E., RITTER, B., 
HEYDER, J. \& SCHULZ, H. 2006. Instillation of six different ultrafine carbon particles indicates a surface area threshold dose for acute lung inflammation in mice. Environ Health Perspect, I I 4, 328-33. STOEHR, L. C., GONZALEZ, E., STAMPFL, A., CASALS, E., DUSCHL, A., PUNTES, V. \& OOSTINGH, G. J. 20I I. Shape matters: effects of silver nanospheres and wires on human alveolar epithelial cells. Part Fibre Toxicol, 8, 36.

STONE, V., BARLOW, P. G., HUTCHISON, G. R. \& BROWN, D. M. 2007. Proinflammatory effects of particles on macrophages and epithelial cells. In: DONALDSON, K. \& BORM, P. (eds.) Particle Toxicology. Boca Raton, FL: CRC Press, Taylor \& Francis Group.

STOPFORD, W., TURNER, J., CAPPELLINI, D. \& BROCK, T. 2003. Bioaccessibility testing of cobalt compounds. J Environ Monit, 5, 675-80.

STRIEGEL, A. M. \& BREWER, A. K. 20 I 2. Hydrodynamic chromatography. Annu Rev Anal Chem (Palo Alto Calif), 5, I 5-34.

SUNG, J. H., JI, J. H., PARK, J. D., SONG, M. Y., SONG, K. S., RYU, H. R., YOON, J. U., JEON, K. S., JEONG, J., HAN, B. S., CHUNG, Y. H., CHANG, H. K., LEE, J. H., KIM, D. W., KELMAN, B. J. \& YU, I. J. 20 I Ia. Subchronic inhalation toxicity of gold nanoparticles. Part Fibre Toxicol, 8, I6.

SUNG, J. H., JI, J. H., PARK, J. D., YOON, J. U., KIM, D. S., JEON, K. S., SONG, M. Y., JEONG, J., HAN, B. S., HAN, J. H., CHUNG, Y. H., CHANG, H. K., LEE, J. H., CHO, M. H., KELMAN, B. J. \& YU, I. J. 2009. Subchronic inhalation toxicity of silver nanoparticles. Toxicol Sci, IO8, 452-6I.

SUNG, J. H., JI, J. H., SONG, K. S., LEE, J. H., CHOI, K. H., LEE, S. H. \& YU, I. J. 20 I I b. Acute inhalation toxicity of silver nanoparticles. Toxicol Ind Health, 27, I 49-54.

SUNG, J. H., JI, J. H., YOON, J. U., KIM, D. S., SONG, M. Y., JEONG, J., HAN, B. S., HAN, J. H., CHUNG, Y. H., KIM, J., KIM, T. S., CHANG, H. K., LEE, E. J., LEE, J. H. \& YU, I. J. 2008. Lung function changes in Sprague-Dawley rats after prolonged inhalation exposure to silver nanoparticles. Inhal Toxicol, 20, 567-74.

SZAKAL, C., ROBERTS, S. M., WESTERHOFF, P., BARTHOLOMAEUS, A., BUCK, N., ILLUMINATO, I., CANADY, R. \& ROGERS, M. 20I4. Measurement of nanomaterials in foods: integrative consideration of challenges and future prospects. ACS Nano, 8, 3 I 28-35.

SZENTKUTI, L. \& LORENZ, K. I995. The thickness of the mucus layer in different segments of the rat intestine. Histochem J, 27, 466-72.

TAKENAKA, S., KARG, E., KREYLING, W. G., LENTNER, B., MOLLER, W., BEHNKESEMMLER, M., JENNEN, L., WALCH, A., MICHALKE, B., SCHRAMEL, P., HEYDER, J. \& SCHULZ, H. 2006. Distribution pattern of inhaled ultrafine gold particles in the rat lung. Inhal Toxicol, I8, 733-40.

TAKENAKA, S., KARG, E., ROTH, C., SCHUlZ, H., ZIESENIS, A., HEINZMANN, U., SCHRAMEL, P. \& HEYDER, J. 200I. Pulmonary and systemic distribution of inhaled ultrafine silver particles in rats. Environ Health Perspect, I09 Suppl 4, 547-5 I.

TAYLOR, A. J., MCCLURE, C. D., SHIPKOWSKI, K. A., THOMPSON, E. A., HUSSAIN, S., GARANTZIOTIS, S., PARSONS, G. N. \& BONNER, J. C. 20 I 4. Atomic layer deposition coating of carbon nanotubes with aluminum oxide alters pro-fibrogenic cytokine expression by human mononuclear phagocytes in vitro and reduces lung fibrosis in mice in vivo. PLoS One, 9, ero6870.

TIEDE, K., BOXALL, A. B. A., WANG, M., GORE, D., TIEDE, D., BAXTER, M., DAVID, H., TEAR, S. P. \& LEWIS, J. 2oIo. Application of Hydrodynamic Chromatography - iCp-ms to Investigate the Fate of Silver Nanoparticles in Activated Sludge. J Anal At Spectrom, 25, I I 49-I I 54. TREUEL, L., JIANG, X. \& NIENHAUS, G. U. 2013. New views on cellular uptake and trafficking of manufactured nanoparticles. J R Soc Interface, Io, 20 I 20939.

TRITSCHER, T., ZERRATH, A. F., ELZEY, S. \& HAN, H. S. 2or3. Data Merging of Size Distributions from Electrical Mobility and Optical Measurements. tsI GmbH, Particle Instruments, Aachen, Germany.

TSUTSUMI, Y. \& YOSHIOKA, Y. 20 I I. Quantifying the biodistribution of nanoparticles. Nat Nanotechnol, 6, 755 . 
ULRICH, A., LOSERT, S., BENDIXEN, N., AL-KATTAN, A., HAGENDORFER, H., NOWACK, B., ADLHART, C., EBERT, J., LATTUADA, M. \& HUNGERBUHLER, K. 2o I 2. Critical Aspects of Sample Handling for Direct Nanoparticle Analysis and Analytical Challenges Using Asymmetric Field Flow Fractionation in a Multi-detector Approach. J Anal At Spectrom, 27, I I 20- I I 30.

VAN DE STOLPE, A. \& DEN TOONDER, J. 20I3. Workshop meeting report Organs-on-Chips: human disease models. Lab Chip, I 3, 3449-70.

VAN DER MEER, A. D. \& VAN DEN BERG, A. 20I2. Organs-on-chips: breaking the in vitro impasse. Integr Biol (Camb), 4, 46I-70.

VAN DER ZANDE, M., VANDEBRIEL, R. J., VAN DOREN, E., KRAMER, E., HERRERA RIVERA, Z., SERRANO-ROJERO, C. S., GREMMER, E. R., MAST, J., PETERS, R. J., HOLLMAN, P. C., HENDRIKSEN, P. J., MARVIN, H. J., PEIJNENBURG, A. A. \& BOUWMEESTER, H. 20 I 2. Distribution, elimination, and toxicity of silver nanoparticles and silver ions in rats after 28-day oral exposure. ACS Nano, 6, 7427-42.

VAN RAVENZWAAY, B., LANDSIEDEL, R., FABIAN, E., BURKHARDT, S., STRAUSS, V. $\&$ MA-HOCK, L. 2009. Comparing fate and effects of three particles of different surface properties: nano- $\mathrm{TiO}(2)$, pigmentary $\mathrm{TiO}(2)$ and quartz. Toxicol Lett, I 86, I 52-9.

VAN SCHOONEVELD, M. M., VUCIC, E., KOOLE, R., ZHOU, Y., STOCKS, J., CORMODE, D. P., TANG, C. Y., GORDON, R. E., NICOLAY, K., MEIJERINK, A., FAYAD, Z. A. \& MULDER, W. J. 2008. Improved biocompatibility and pharmacokinetics of silica nanoparticles by means of a lipid coating: a multimodality investigation. Nano Lett, 8, 25 I7-25.

VANDEBRIEL, R. J. \& DE JONG, W. H. 20I2. A review of mammalian toxicity of $\mathrm{ZnO}$ nanoparticles. Nanotechnol Sci Appl, 5, 6I-7I.

VARGHESE, S. K. \& GANGAMMA, S. 2009. Particle deposition in human respiratory system: deposition of concentrated hygroscopic aerosols. Inhal Toxicol, 2 I, 619-30.

VASCO, F., HAWE, A. \& JISKOOT, W. 20го. Critical Evaluation of Nanoparticle Tracking Analysis (ntA) by NanoSight for the Measurement of Nanoparticles and Protein Aggregates. Pharm Res, 27, 796-8 Io.

VERANTH, J. M., GHANDEHARI, H. \& GRAINGER, D. W. 20Iо. Nanoparticles in the Lung. Comprehensive Toxicology. Elsevier.

VINCENT, J. H., JOHNSTON, A. M., JONES, A. D., BOLTON, R. E. \& ADDISON, J. I985. Kinetics of deposition and clearance of inhaled mineral dusts during chronic exposure. $\mathrm{Br} J$ Ind Med, 42, 707-I 5 .

VON DER KAMMER, F., LEGROS, S., HOFMANN, T., LARSEN, E. H. \& LOESCHNER, K. 20 I I. Separation characterization of nanoparticles in complex food and environmental samples by field-flow fractionation. Trends in Analytical Chemistry, 30, 425-436.

WAGNER, I., MATERNE, E. M., BRINCKER, S., SUSSBIER, U., FRADRICH, C., BUSEK, M., SONNTAG, F., SAKHAROV, D. A., TRUSHKIN, E. V., TONEVITSKY, A. G., LAUSTER, R. \& MARX, U. 20I3. A dynamic multi-organ-chip for long-term cultivation and substance testing proven by $3 \mathrm{D}$ human liver and skin tissue co-culture. Lab Chip, I3, 3538-47.

WAHLUND, K. G. \& GIDDINGS, J. C. 1987. Properties of An Asymmetrical Flow Field-flow Fractionation Channel Having One Permeable Wall. Anal Chem, 59, I332-I 339.

WALCZAK, A. P., KRAMER, E., HENDRIKSEN, P. J., TROMP, P., HELSPER, J. P., VAN DER ZANDE, M., RIETJENS, I. M. \& BOUWMEESTER, H. 20I4. Translocation of differently sized and charged polystyrene nanoparticles in in vitro intestinal cell models of increasing complexity. Nanotoxicology, I-9.

WALLACE, W. E., KEANE, M. J., MIKE, P. S., HILL, C. A., VALLYATHAN, V. \& REGAD, E. D. 1992. Contrasting respirable quartz and kaolin retention of lecithin surfactant and expression of membranolytic activity following phospholipase A2 digestion. J Toxicol Environ Health, 37, 391-409. WALLENBORN, J. G., MCGEE, J. K., SCHLADWEILER, M. C., LEDBETTER, A. D. \& KODAVANTI, U. P. 2007. Systemic translocation of particulate matter-associated metals following a single intratracheal instillation in rats. Toxicol Sci, 98, 23 I-9. 
WALSER, T. \& STUDER, C. 2015. Sameness: The regulatory crux with nanomaterial identity and grouping schemes for hazard assessment. Regul Toxicol Pharmacol, 72, 569-57I.

WANG, J., ZHOU, G., CHEN, C., YU, H., WANG, T., MA, Y., JIA, G., GAO, Y., LI, B., SUN, J., LI, Y., JIAO, F., ZHAO, Y. \& CHAI, Z. 2007. Acute toxicity and biodistribution of different sized titanium dioxide particles in mice after oral administration. Toxicol Lett, I68, I76-85.

WANG, L., DING, W. \& ZHANG, F. 20I0. Acute toxicity of ferric oxide and zinc oxide nanoparticles in rats. J Nanosci Nanotechnol, Io, 8617-24.

WANG, M., ZHENG, L. N., WANG, B., CHEN, H. Q., ZHAO, Y. L., CHAI, Z. F., REID, H. J., SHARP, B. L. \& FENG, W. Y. 20 I 4a. Quantitative analysis of gold nanoparticles in single cells by laser ablation inductively coupled plasma-mass spectrometry. Anal Chem, 86, I0252-6.

WANG, X., JI, Z., CHANG, C. H., ZHANG, H., WANG, M., LIAO, Y. P., LIN, S., MENG, H., LI, R., SUN, B., WINKLE, L. V., PINKERTON, K. E., ZINK, J. I., XIA, T. \& NEL, A. E. $20 \mathrm{I}$ b. Use of coated silver nanoparticles to understand the relationship of particle dissolution and bioavailability to cell and lung toxicological potential. Small, го, 385-98.

WANG, Z., CHEN, J., LI, X., SHAO, J. \& PEIJNENBURG, W. J. 20I2. Aquatic toxicity of nanosilver colloids to different trophic organisms: contributions of particles and free silver ion. Environ Toxicol Chem, 31, 2408-I 3.

WARHEIT, D. B., HOKE, R. A., FINLAY, C., DONNER, E. M., REED, K. L. \& SAYES, C. M. 2007a. Development of a base set of toxicity tests using ultrafine $\mathrm{TiO}_{2}$ particles as a component of nanoparticle risk management. Toxicol Lett, I7 I, 99-I Io.

WARHEIT, D. B., WEBB, T. R., COLVIN, V. L., REED, K. L. \& SAYES, C. M. 2007b. Pulmonary bioassay studies with nanoscale and fine-quartz particles in rats: toxicity is not dependent upon particle size but on surface characteristics. Toxicol Sci, 95, 270-80.

WARHEIT, D. B., WEBB, T. R., REED, K. L., FRERICHS, S. \& SAYES, C. M. 2007c. Pulmonary toxicity study in rats with three forms of ultrafine- $\mathrm{TiO}_{2}$ particles: differential responses related to surface properties. Toxicology, 230, 90-104.

WARHEIT, D. B., WEBB, T. R., SAYES, C. M., COLVIN, V. L. \& REED, K. L. 2006. Pulmonary instillation studies with nanoscale $\mathrm{TiO}_{2}$ rods and dots in rats: toxicity is not dependent upon particle size and surface area. Toxicol Sci, 91, 227-36.

WHITESIDES, G. M. 2006. The origins and the future of microfluidics. Nature, 442, 368-73.

WIJNHOVEN, S. W. P., DEKKERS, S., KOOI, M., JONGENEEL, R. \& JONG, W. H. 2 oı . Nanomaterials in consumer products : Update of products on the European market in 20IO. RIVM Rapport 340370003.

WIJNHOVEN, S. W. P., PEIJNENBURG, W. J. G. M., HERBERTS, C. A., HAGENS, W. I., OOMEN, A. G., HEUGENS, E. H. W., ROSZEK, B., BISSCHOPS, J., GOSENS, I., MEENT, D. V. D., DEKKERS, S., JONG, W. H. D., ZIJVERDEN, M. V., SIPS, A. J. A. M. \& GEERTSMA, R. E. 2009. Nano-silver - a review of available data and knowledge gaps in human and environmental risk assessment. Nanotoxicology, 3, 36.

WITTMAACK, K. 2007. In search of the most relevant parameter for quantifying lung inflammatory response to nanoparticle exposure: particle number, surface area, or what? Environ Health Perspect, I I 5 , I 87-94.

WOLFF, A., ANTFOLK, M., BRODIN, B. \& TENJE, M. 20I5. In Vitro Blood-Brain Barrier Models-An Overview of Established Models and New Microfluidic Approaches. J Pharm Sci.

WORTH, A. P. \& BALLS, M. 2004. The principles of validation and the ECVAM validation process. Altern Lab Anim, 32 Suppl I B, 623-9.

WWW.NANOGENOTOX.COM. 20I2. Report from work package 7: Toxicokinetics and tissue distribution of Mns for specification of organs at risk for genotoxicity testing [Online]. Available: www.nanogenotox.com [Accessed 30-06-20I2.

XIA, T., KOVOCHICH, M., BRANT, J., HOTZE, M., SEMPF, J., OBERLEY, T., SIOUTAS, C., YEH, J. I., WIESNER, M. R. \& NEL, A. E. 2006. Comparison of the abilities of ambient and manufactured nanoparticles to induce cellular toxicity according to an oxidative stress paradigm. Nano Lett, 6, I794-807. 
XIA, T., KOVOCHICH, M., LIONG, M., ZINK, J. I. \& NEL, A. E. 2008. Cationic polystyrene nanosphere toxicity depends on cell-specific endocytic and mitochondrial injury pathways. ACS Nano, 2, 85-96.

XIE, Y., WILLIAMS, N. G., TOLIC, A., CHRISLER, W. B., TEEGUARDEN, J. G., MADDUX, B. L., POUNDS, J. G., LASKIN, A. \& ORR, G. 20I2. Aerosolized ZnO nanoparticles induce toxicity in alveolar type II epithelial cells at the air-liquid interface. Toxicol Sci, I 25, 450-6I.

XIU, Z. M., ZHANG, Q. B., PUPPALA, H. L., COLVIN, V. L. \& ALVAREZ, P. J. 20 I 2. Negligible particle-specific antibacterial activity of silver nanoparticles. Nano Lett, I 2, 427 I-5.

YACOBI, N. R., DEMAIO, L., XIE, J., HAMM-ALVAREZ, S. F., BOROK, Z., KIM, K. J. \& CRANDALL, E. D. 2008. Polystyrene nanoparticle trafficking across alveolar epithelium. Nanomedicine, 4, I39-45.

YANG, W., PETERS, J. I. \& WILLIAMS, R. O., 3 RD 2008. Inhaled nanoparticles--a current review. Int J Pharm, 356, 239-47.

YAZDI, A. S., GUARDA, G., RITEAU, N., DREXLER, S. K., TARDIVEL, A., COUILLIN, I. \& TSCHOPP, J. 20I0. Nanoparticles activate the NLR pyrin domain containing 3 (Nlrp3) inflammasome and cause pulmonary inflammation through release of Il-Ialpha and Il-Ibeta. Proc Natl Acad Sci U S A, I07, I9449-54.

YEH, H. C., SCHUM, G. M. \& DUGGAN, M. T. I979. Anatomic models of the tracheobronchial and pulmonary regions of the rat. Anat Rec, 195, 483-92.

YU, L. E., YUNG, L.-Y. L., ONG, C.-N., TAN, Y.-L., BALASUBRAMANIAM, K. S., HARTONO, D., SHUI, G., WENK, M. R. \& ONG, W.-Y. 2007. Translocation and effects of gold nanoparticles after inhalation exposure in rats. Nanotoxicology, I, 23 5-242.

ZATTONI, A., RAMBALDI, D. C., RODA, B., PARISI, D., RODA, A., MOON, M. H. \& RESCHIGLIAN, P. 2008. Hollow-fiber flow field-flow fractionation of whole blood serum. $J$ Chromatogr $A$, I I 83 , I $35-42$.

ZATTONI, A., RODA, B., BORGHI, F., MARASSI, V. \& RESCHIGLIAN, P. 20I4. Flow fieldflow fractionation for the analysis of nanoparticles used in drug delivery. J Pharm Biomed Anal, 87, 53-6I.

ZHANG, L. W., BAUMER, W. \& MONTEIRO-RIVIERE, N. A. 20 i r. Cellular uptake mechanisms and toxicity of quantum dots in dendritic cells. Nanomedicine (Lond), 6, 777-9I.

ZHANG, Q., KUSAKA, Y., SATO, K., NAKAKUKI, K., KOHYAMA, N. \& DONALDSON, K. I998. Differences in the extent of inflammation caused by intratracheal exposure to three ultrafine metals: role of free radicals. $J$ Toxicol Environ Health $A$, 53, 423-38.

ZHANG, T., WANG, L., CHEN, Q. \& CHEN, C. 20I4. Cytotoxic potential of silver nanoparticles. Yonsei Med J, 55, 283-91.

ZHANG, Z., KONG, F., VARDHANABHUTI, B., MUSTAPHA, A. \& LIN, M. 20 I 2. Detection of engineered silver nanoparticle contamination in pears. J Agric Food Chem, 60, 10762-7.

ZHOU, X., DAI, Q. \& HUANG, X. 20I 2. Neutrophils in acute lung injury. Front Biosci (Landmark $E d),{ }_{17}, 2278-83$.

ZHU, J., ROGERS, A. V., BURKE-GAFFNEY, A., HELLEWELL, P. G. \& JEFFERY, P. K. I 999. Cytokine-induced airway epithelial ICAM-I upregulation: quantification by high-resolution scanning and transmission electron microscopy. Eur Respir J, I3, I 3 I 8-28.

ZHU, M. T., FENG, W. Y., WANG, B., WANG, T. C., GU, Y. Q., WANG, M., WANG, Y., OUYANG, H., ZHAO, Y. L. \& CHAI, Z. F. 2008. Comparative study of pulmonary responses to nano- and submicron-sized ferric oxide in rats. Toxicology, 247, I02-I I.

ZOOK, J. M., LONG, S. E., CLEVELAND, D., GERONIMO, C. L. \& MACCUSPIE, R. I. 20 I I. Measuring silver nanoparticle dissolution in complex biological and environmental matrices using UV-visible absorbance. Anal Bioanal Chem, 401, 1993-2002. 



\section{Dankwoord}

Het is zover, mijn boekje is af? Met veel plezier heb ik de afgelopen jaren aan dit proefschrift gewerkt, maar dit heb ik niet alleen gedaan. Vele mensen hebben mij geholpen, wijze raad gegeven, aangemoedigd of juist afgeleid om te kunnen ontspannen. Daar ben ik erg dankbaar voor. Ik wil in het bijzonder de volgende mensen bedanken.

Henk, als promotor heb je mij geholpen om de grote lijn van het proefschrift vast te houden. Met je scherpe vragen en doordringende blik heb je mij geleerd om kritisch naar onderzoek te kijken. Je vertrouwen heeft mij gevormd tot zelfstandig onderzoeker. Daarnaast stond je altijd voor me klaar als ik advies nodig had, dat heb ik erg gewaardeerd. Bedankt!

Margriet, met jou als co-promotor en begeleider heb ik veel geluk gehad. Doordat mijn onderzoek in het verlengde lag van jouw promotieonderzoek, was je voor mij een ideale vraagbaak en sparringpartner. Dankzij jou heb ik geleerd om de focus in mijn onderzoek vast te houden en doordachte keuzes te maken. Onze samenwerking heeft ervoor gezorgd dat mijn onderzoek soepel verliep. Je gaf me veel vrijheid om mijn gang te gaan, maar op de juiste momenten liet je me gas terugnemen met kritische kanttekeningen. Heel erg bedankt dat ik altijd bij je aan mocht kloppen voor vragen, advies of gewoon om een praatje te maken. Ik hoop dat onze soepele en ook gezellige samenwerking nog lang blijft bestaan.

Flemming, als expert op het gebied van de inhalatie toxicologie heb je mij geholpen bij het schrijven van een aantal artikelen. In het begin was ik soms benauwd voor je kritiek, maar naarmate ik verder in mijn onderzoek kwam waardeerde ik dat juist. Ik heb enorm veel van je kritische opmerkingen geleerd. Bij ieder stuk tekst wist jij de pijnpunten te vinden, waardoor ik artikelen kon verbeteren. Bedankt voor je hulp de afgelopen jaren! In de toekomst hoop ik nog vaak gebruik te maken van je inzichten.

Wim, jij hebt ervoor gezorgd dat het IRAN project (Integrated Risk Assessment of Nanomaterials) werd gestart bij het RIVM. Je was altijd erg enthousiast en betrokken bij mijn onderzoek. Met een achtergrond als patholoog had je veel belangstelling voor de details van het onderzoek. Zelfs de microscoop op je kamer hebben we afgestoft om naar longcoupes te kijken. Bedankt voor je betrokkenheid!

Rob, Ilse, Agnes, Adrienne, Willie, Susan en alle anderen binnen de nanotox-groep wil ik bedanken voor de adviezen en hulp bij het uitvoeren en opschrijven van de verschillende studies. Dankzij jullie verschillende invalshoeken heb ik resultaten van allerlei kanten kunnen belichten. 
Jolanda, Eric, Liset, Henny, Paul en John, bedankt voor jullie hulp bij de experimenten. Miljarden cellen, miljoenen nanodeeltjes, honderden inserts, tientallen dieren en testen verder is het gelukt om meer te weten te komen over de effecten van nanomaterialen. Ik vind het erg fijn om met jullie samen te werken en hoop dat we dit nog wat jaren zullen doen!

Jochem, Kirsten, Anne, Sjors, Christina, Sander, Linda, Myrto, Charlotte, Tessel, Tessa en alle andere (voormalige) bewoners van A2.I I9 en A2.I20 wil ik bedanken voor de gezelligheid op de gang en op de kamer. Dankzij jullie ging ik met veel plezier naar mijn werk en kon ik altijd mijn verhaal kwijt. Jochem en Kirsten, naast werk delen we de passie voor spellen en puzzels (liefst met een biertje erbij). Samen kunnen we ieder record verbreken. Bedankt dat jullie mijn paranimfen willen zijn!

Alle andere collega's binnen GZB wil ik bedanken voor de gezellige tijd en goede sfeer de afgelopen jaren. Dankzij borrels, filmavond, sport- en speldag, kerst en lab-uitjes had en heb ik het ontzettend naar mijn zin!

Vrienden die mij hebben geholpen om te zien dat er meer is in het leven dan 'nano', bedankt! Hanne en Maartje, bedankt dat ik tegen jullie aan kan kletsen tijdens het hardlopen. Hydrofielers en Hydroladiezzzz (en aanhang natuurlijk), zonder zwembad vind ik het nog leuker met jullie! Bedankt voor de gezellige avondjes en weekendjes weg, ik hoop dat we onze tradities voortzetten. En Inge, op naar een volgend jubileum! Oud-PJK en GeNoOTen bedankt voor jullie vriendschap. We gaan al heel wat jaren terug en hopelijk nog veel meer in de toekomst! Bedankt voor de ontspanning en gekkigheid tijdens het zingen en daarbuiten.

Familie die al mijn verhalen over nanodeeltjes, experimenten en collega's moesten aanhoren, bedankt voor jullie interesse en ondersteuning. Frans en Til bedankt voor jullie interesse in mijn onderzoek en jullie hulp bij het verduidelijken van de Nederlandse samenvatting. Myriam, bedankt voor je luisterend oor en de wetenschap dat je er altijd voor me bent. Papa, Esther, Mama en Theo, bedankt dat ik altijd bij jullie terecht kan. Jullie wilden echt begrijpen waar ik het over had en dat waardeer ik erg. Tijdens het proppen of een avondje film kijken kunnen we alles bespreken. Dankzij jullie voel ik mij gesteund. Bedankt!

Thijs, ik weet niet waar ik moet beginnen om jou te bedanken. Bij jou kan ik mij ontspannen en voel ik mij thuis. Over mijn onderzoek hadden we leuke discussies, jij vindt altijd een nieuwe manier om te kijken. Bedankt voor je aandacht, geduld, grappen en gezelligheid. Bedankt voor alles! X 




\section{List of publications}

BRAAKHUIS, H. M., GIANNAKOU, C., PEIJNENBURG, W. J. G. M., VERMEULEN, J., VAN LOVEREN, H. \& PARK, M. V. D. Z. 20I 5 d. Simple in vitro models can predict pulmonary toxicity of silver nanoparticles. Nanotoxicology, accepted Nov 2015.

BRAAKHUIS, H.M., OOMEN, A.G. \& CASSEE, F.R. 20I 5 c. Grouping nanomaterials to predict their potential to induce pulmonary inflammation. Toxicol Appl Pharmacol, Special issue "The First Decade of Nanotoxicology: Achievements, Disappointments and Lessons".

BRAAKHUIS, H. M., KLOET, S. K., KEZIC, S., KUPER, F., PARK, M. V., BELLMANN, S., VAN DER ZANDE, M., LE GAC, S., KRYSTEK, P., PETERS, R. J., RIETJENS, I. M. \& BOUWMEESTER, H. 20 I $5 \mathrm{~b}$. Progress and future of in vitro models to study translocation of nanoparticles. Arch Toxicol, 89, 1469-95.

BRAAKHUIS, H. M., CASSEE, F. R., FOKKENS, P. H., DE la FONTEYNE, L. J., OOMEN, A. G., KRYSTEK, P., DE JONG, W. H., VAN LOVEREN, H. \& PARK, M. V. 20I 5a. Identification of the appropriate dose metric for pulmonary inflammation of silver nanoparticles in an inhalation toxicity study. Nanotoxicology, I-I I.

BRAAKHUIS, H. M., GOSENS, I., KRYSTEK, P., BOERE, J., CASSEE, F. R., FOKKENS, P., POST, J., VAN LOVEREN, H. \& PARK, M. 20 I 4b. Particle size dependent deposition and pulmonary inflammation after short-term inhalation of silver nanoparticles. Part Fibre Toxicol, II, I6.

BRAAKHUIS, H. M., PARK, M. V., GOSENS, I., DE JONG, W. H. \& CASSEE, F. R. 20I4a. Physicochemical characteristics of nanomaterials that affect pulmonary inflammation. Part Fibre Toxicol, I I, I8.

KRYSTEK, P., BRAAKHUIS, H. M., PARK, M. V. D. Z. \& JONG, W. H. D. 20I3. Inductively Coupled Plasma-Mass Spectrometry in Biodistribution Studies of (Engineered) Nanoparticles. Encyclopedia of Analytical Chemistry. I 5 March 2013 ed.: John Wiley \& Sons, Ltd.

ZWART, E. P., SCHAAP, M. M., VAN DEN DUNGEN, M. W., BRAAKHUIS, H. M., WHITE, P. A., VAN STEEG, H., VAN BENTHEM, J. \& LUIJTEN, M. 20I 2. Proliferating primary hepatocytes from the pUR288 lacZ plasmid mouse are valuable tools for genotoxicity assessment in vitro. Environ Mol Mutagen, 53, I-8. 



\section{Curriculum vitae}

Hedwig Magdalena Brakhuis werd geboren op 2 januari 1987 te Heerenveen. Na een jeugd in Vriezenveen behaalde ze in 2005 haar Gymnasium diploma aan College De Klop in Utrecht. Aansluitend studeerde zij biomedische wetenschappen aan de Radboud Universiteit Nijmegen, met als minor Publiek Management en als hoofdvak Toxicologie. Ze liep stage bij Royal Haskoning in Nijmegen waar ze de interpretatie van de cosmeticawetgeving binnen de chemische stoffen wetgeving REACH onderzocht. Als hoofdvakstage hielp ze bij het RIVM mee aan de ontwikkeling van een assay om genotoxiciteit van stoffen te detecteren in primaire hepatocyten. In 20 Io behaalde ze haar master diploma en werd ze aangenomen bij Royal Haskoning als Junior Toxicoloog waar zij zich bezighield met de implementatie van REACH. In 20 I I startte zij haar onderzoek naar de risico's van nanomaterialen als AIO bij het RIVM. Tijdens dit onderzoek voltooide zij de Postdoctorale Opleiding in Toxicologie, dat zal resulteren in een Europese registratie als toxicoloog. Sinds 2015 werkt zij als wetenschappelijk onderzoeker bij het RIVM.

Hedwig Magdalena Braakhuis was born in Heerenveen on the 2nd of January 1987. After growing up in Vriezenveen she graduated in 2005 her secondary education at College De Klop in Utrecht. In the same year, she started her study Biomedical Sciences at the Radboud University Nijmegen with a minor in Public Management and a major in Toxicology. During her study, she conducted a master internship at the department of Chemicals Management at Royal Haskoning, Nijmegen. This internship focused on the interpretation of the Cosmetics Legislation within REACH. She conducted a second master internship at the centre for health protection at the RIVM where she developed an assay to detect genotoxicity of chemical substances in primary hepatocytes. In 2010 , she obtained het master's degree and started as a Junior Toxicologist at Royal Haskoning, Nijmegen. In 20 I I, she started as a PhD at the RIVM where she investigated the potential risks of nanomaterials after inhalation. During this research period, she completed the Postgraduate Education in Toxicology, which will result in a European registration as a toxicologist. In 2015 , she is working as a scientific researcher at the RIVM. 
